\title{
Identifying the North American Plum Species Phylogenetic Signal Using Nuclear, Mitochondrial, and Chloroplast DNA Markers
}

\author{
Dario J. Chavez ${ }^{1}$ \\ Department of Horticulture, University of Georgia, Griffin Campus, Griffin, GA 30223 \\ Thomas G. Beckman \\ USDA-Agricultural Research Service, Southeastern Fruit and Tree Nut Research Laboratory, Byron, \\ GA 31008 \\ José X. Chaparro
Horticultural Sciences Department, University of Florida, Gainesville, FL 32611
}

AdDitional Index words. cpDNA, haploids, ITS, nuclear genes, phylogenetic analyses, PICS, section Prunocerasus, single-copy gene, SSRs

\begin{abstract}
Prunus phylogeny has been extensively studied using chloroplast DNA (cpDNA) sequences. Chloroplast DNA has a slow rate of evolution, which is beneficial to determine species relationships at a deeper level. The chloroplast-based phylogenies have a limitation due to the transfer of this organelle by interspecific hybridization. This creates difficulties when studying species relationships. Interspecific hybrids in Prunus occur naturally and have been reported, which creates a problem when using cpDNA-based phylogenies to determine species relationships. The main goal of this project was to identify nuclear gene regions that could provide an improved phylogenetic signal at the species level in Prunus. A total of 11 species in Prunus and within section Prunocerasus were used. Two peach (Prunus persica) haploids were used to test the reliability of the molecular markers developed in this project to amplify single-copy genes. A total of 33 major genes associated with vernalization response, 16 with tree architecture, and 3 with isozymes, were tested. Similarly, 41 simple sequence repeat (SSR) markers, seven cpDNA regions, and the internal transcribed spacer (ITS) region, were used. Multiple gene regions were identified and provided the greatest number of characters, greatest variability, and improved phylogenetic signal at the species level in Prunus section Prunocerasus. Out of those, trnH-psbA, PGI, MAX4, AXR1, LFY, PHYE, and VRN1 are recommended for a phylogenetic analysis with a larger number of taxa. The use of potentially informative characters (PICS) as a measure of how informative a region will be for phylogenetic analyses has been previously reported beneficial in cpDNA regions and it clearly was important in this research. This will allow selecting the region(s), which can be used in phylogenetic studies with higher number of taxa.
\end{abstract}

Taxonomic conflicts in Prunus were reported by Waugh (1901) and Hedrick (1911). Waugh (1901) stated that "plums grow pretty much as they please, and the botanist has to take them as he finds them." He recognized that new classifications sometimes changed the previously published species groupings and classifications. Prunus classifications and discrepancies were well summarized by Bortiri et al. (2001), with Rehder's (1940) classification being recognized as the most widely used and accepted. Rehder's (1940) classification divided Prunus into five subgenera: Prunophora (Prunus), Amygdalus, Cerasus, Padus, and Laurocerasus. The subgenus Prunus was further divided into sections: Euprunus, Prunocerasus (North American plums), and Armeniaca. Recent phylogenetic studies

\footnotetext{
Received for publication 11 July 2016. Accepted for publication 13 Oct. 2016. We thank Wayne Sherman, Pamela Soltis, Doug Soltis, Matias Kirst, Jim Olmstead, and Kent Perkins (University of Florida) and Dick Okie (USDAARS) for their support and knowledge provided for this project. We also thank a large group of collaborators in multiple private and public institutions who helped before, during, and after specimen collections. They also extend their thanks to the USDA Prunus Germplasm Collection, its director and curators, and the Prunus Germplasm Committee for their financial support for the germplasm collection used in this project, USDA-ARS project No. 5306-21000018-00D

${ }^{1}$ Corresponding author. E-mail: dchavez@uga.edu.
}

supported the concept of Prunus as a monophyletic group (single genus). However, the genus Prunus contained several poorly supported subclades/terminals (subgenera/species) (Bortiri et al., 2001, 2002, 2006; Kaneko et al., 1986; Katayama and Uematsu, 2005; Lee and Wen, 2001; Shaw and Small, 2004, 2005; Wen et al., 2008).

The genus Prunus is globally distributed, with $\approx 200$ species. North America is an important center of diversity for plum species adapted to divergent climates and soils. Plums are considered to harbor the greatest diversity of fruit flavor, aroma, texture, color, form, and size among the stone fruits (Hedrick, 1911; Waugh, 1901). Mason's (1913) and Rehder's (1940) taxonomic classifications have been used for most of the Prunus phylogenetic analyses. Hereafter, the North American plums will be referred to as section Prunocerasus (Bortiri et al., 2001; Shaw and Small, 2004).

The phylogeny of the genus Prunus was studied by Mowrey and Werner (1990) using isozymes. Prunus section Prunocerasus was found to be polyphyletic, with a clade composed of Prunus americana, Prunus munsoniana, Prunus hortulana, Prunus subcordata, and Prunus angustifolia, and with Prunus maritima and Prunus umbellata in another. Badenes and Parfitt (1995) reported similar results to Mowrey and Werner (1990) using cpDNA sequences. They recovered all conventional 
subgenus classifications based on Rehder's (1940) taxonomic treatment: P. persica-Prunus dulcis, Prunus domestica-Prunus salicina, and Prunus cerasus-Prunus fruticosa monophyletic clades.

Two major groups, the Amygdalus-Prunus group and the Cerasus-Laurocerasus-Padus group, were recovered by Lee and Wen's (2001) phylogenetic analysis using ITS sequences of ribosomal DNA. These results were not congruent with Rehder's (1940) taxonomic treatment. Bortiri et al. (2001) supported the genus Prunus monophyly using ITS and chloroplast trnL-trnF spacer DNA sequences. Subgenera Padus-Laurocerasus-Cerasus and subgenera Prunus-Amygdalus-Emplectocladus-Cerasus (section Microcerasus)-section Penarmeniaca formed two major clades, respectively. The plums of northeastern North America were found to be closely related with Prunus mexicana as sister to the rest of this clade.

Bortiri et al. (2002) used the nuclear gene sorbitol 6phosphate dehydrogenase $(s 6 p d h)$ combined with ITS and trnL-trnF sequences to improve the lack of definition for deep nodes in the subgenera Prunus-Amygdalus-Emplectocladus clade, as previously reported (Bortiri et al., 2001). Phylogenetic analysis of the combined data supported two major clades: subgenera Cerasus-Laurocerasus-Padus and subgenera AmygdalusEmplectocladus-Prunus. Section Microcerasus (subgenus Cerasus) was found nested within subgenus Prunus.

Prunus subgenus Prunus section Prunocerasus was reported to be monophyletic by Shaw and Small (2004) based on the data from seven cpDNA regions: $r p S 16, \operatorname{rpL16}$, trnL, trn $G$, trnL$\operatorname{trnF}, \operatorname{trnS}$-trnG, and trnH-psbA. Three clades were strongly supported in section Prunocerasus based on Waugh's (1901) classification: "American Clade," "Chickasaw Clade," and "Beach Clade." The American Clade included P. americana var. americana, $P$. americana var. lanata, $P$. mexicana, Prunus rivularis, $P$. hortulana, $P$. umbellata var. injucunda; the Chickasaw Clade included $P$. angustifolia, P. munsoniana, Prunus gracilis, Prunus nigra, P. umbellata var. umbellata, Prunus alleghaniensis var. alleghanienses, and P. alleghaniensis var. davisii; and the Beach Clade included Prunus geniculata, $P$. maritima var. maritima, and $P$. maritima var. gravesii.

The majority of phylogenetic research in Prunus has been done using cpDNA sequences (Badenes and Parfitt, 1995; Bortiri et al., 2001, 2002, 2006; Kaneko et al., 1986; Katayama and Uematsu, 2005; Shaw and Small, 2004, 2005; Wen et al., 2008). The advantages and disadvantages of using cpDNA for plant phylogenetic analyses have been well summarized by Soltis and Soltis (1998). One of the advantages includes that the chloroplast genome of plants is small. The size of the peach (cv. Hakuhou) chloroplast genome has been estimated to be about $152 \mathrm{~kb}$ (Katayama and Uematsu, 2005). The chloroplast genome rate of evolution is considered to be slow in comparison with nuclear genes (Soltis and Soltis, 1998). The latter could be considered an advantage when studying distantly related species and disadvantage when studying closely related species and species at the population level. The maternal inheritance of chloroplasts can be used to track interspecific introgression events, but it could lead to erroneous species relationships (Soltis and Soltis, 1998).

The slow rate of evolution of several cpDNA sequences and their value to infer phylogenetic relationships in closely related species for some angiosperms, including Prunus, was revised by Small et al. (1998) and Shaw et al. (2005, 2007). The main objective of the study of Shaw et al. (2007) was to identify chloroplast regions that would provide the greatest number of characters for low-level molecular phylogenetic studies. Shaw et al. (2007) recommended selecting the top few choices with the greatest variability to be screened in a particular lineage to determine which of those are the most informative for a specific group.

Bortiri et al. (2006) demonstrated the power of including morphological characters for Prunus phylogenetic analyses. The combined data provided by ITS sequences, cpDNA regions, and morphological characters for Prunus yielded support of some nodes that were previously identified in Prunus (Bortiri et al., 2001). Several synapomorphies supported large groups, providing additional resolution for some clades. Similarly, Rohrer et al. (2004) reported that microsatellite markers could provide the genetic variability necessary to resolve the relationships within Prunus at the species level.

The main objective of this research was to measure and to identify additional genomic regions that could provide the greatest number of characters, greatest variability, and improved phylogenetic signal at the species level for Prunus section Prunocerasus relationships.

\section{Materials and Methods}

\section{Plant material}

A total of 11 species, one accession per taxa, were collected from the wild (excluding $P$. persica haploids) by T.G. Beckman, W.R. Okie, D.J. Chavez, and J.X. Chaparro (Table 1). A total of 13 samples (including two peach haploids) were used for this study. These species represented outgroups and major clades within subgenus Prunus section Prunocerasus, as reported by Shaw and Small (2004). The American Clade was represented by $P$. americana, $P$. hortulana, and $P$. mexicana; the Chickasaw Clade included $P$. angustifolia, $P$. munsoniana, and $P$. umbellata; and the Beach Clade was represented by $P$. geniculata and P. maritima. Outgroups included Prunus pumila (subgenus Prunus section Penarmeniaca); P. persica cv. Okinawa, AP0518ws (haploid peach selection), 02-01c (haploid peach selection) (subgenus Amygdalus); and P. fasciculata (subgenus Emplectocladus). Peach haploids were used for confirmation of sequencing products.

The plant specimens collected were deposited as herbarium vouchers at the University of Florida Herbarium in the Florida Museum of Natural History, Gainesville, FL. Additional leaf material from the collected samples were freeze dried (FreeZone 2.5 L system; Labconco, KS City, MO) or dried using a mixture of $2.5 \mathrm{~kg} 28$ - to 200-mesh silica gel dessicant (catalog no. S157-212; Thermo Fisher Scientific, Waltham, MA) and $500 \mathrm{~g} \mathrm{10}$ - to 18-mesh silica gel dessicant (catalog no. S161-500, Tel-Tale ${ }^{\mathrm{TM}}$; Thermo Fisher Scientific). Dried sample materials are stored at the Stone Fruit Breeding and Genetics Program at the University of Florida, Gainesville, FL.

\section{DNA isolation}

DNA was extracted from leaf tissue using a modified cetyltrimethylammonium bromide (CTAB) method as described by Chavez and Chaparro (2011). Lyophilized leaf tissue $(\approx 10 \mathrm{mg}$ ) of each sample was added to $2-\mathrm{mL}$ Eppendorf microcentrifuge tubes with three 5-mm stainless-steel beads, $750 \mu \mathrm{L}$ of CTAB buffer [2\% CTAB, $100 \mathrm{~mm}$ Tris $\mathrm{pH} 8.0,1.4 \mathrm{M}$ $\mathrm{NaCl}, 0.5 \mathrm{M}$ ethylenediaminetetraacetic acid (EDTA), 1\% polyvinylpyrrolidone] previously mixed with mercaptoethanol 


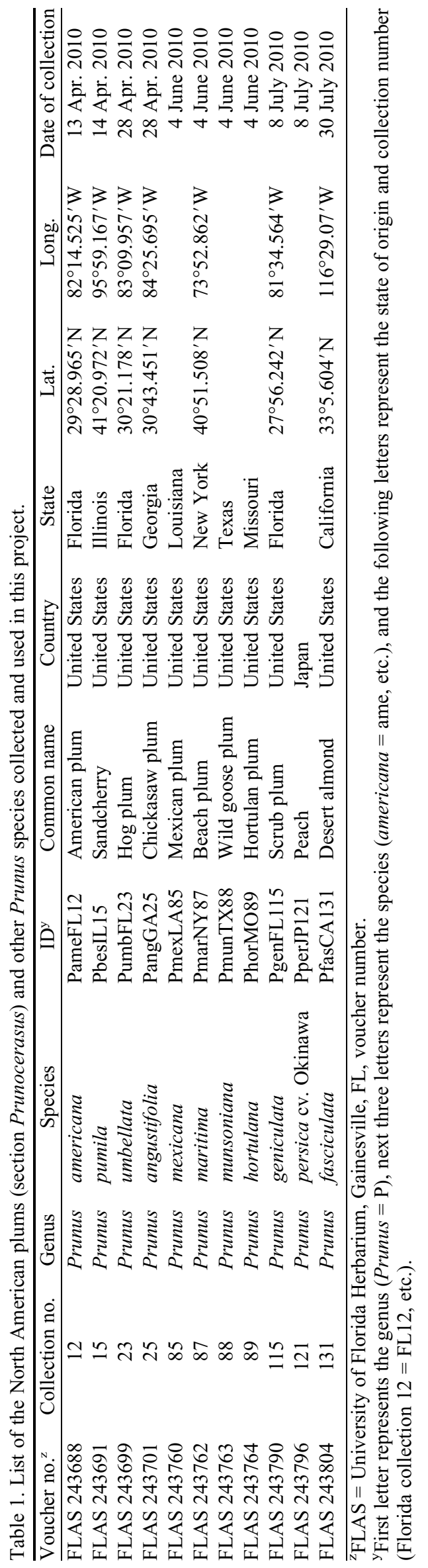

$\left(1 \mu \mathrm{L} \cdot \mathrm{mL}^{-1}\right)$, and $8 \mu \mathrm{L}$ of RNAse $\left(10 \mathrm{mg} \cdot \mathrm{mL}^{-1}\right)$. Samples were grounded two/three times at $30 \mathrm{~Hz}$ in a tissue lyser (Qiagen, Valencia, CA) for 1.5 min until tissue clumps were not visible. Samples were vortexed and incubated in a $65^{\circ} \mathrm{C}$ water bath for $6 \mathrm{~min}$. Then, tubes were vortexed and a volume of $750 \mu \mathrm{L}$ of chloroform:isoamyl (24:1) was added. Tubes were vortexed, incubated at $-20^{\circ} \mathrm{C}$ for $6 \mathrm{~min}$, and then centrifuged at $12,000 \mathrm{~g}_{\mathrm{n}}$ for $10 \mathrm{~min}$. The aqueous phase was transferred to a new $2-\mathrm{mL}$ centrifuge tube, and $500 \mu \mathrm{L}$ of cold isopropanol was added. Tubes were gently mixed, incubated at $-20{ }^{\circ} \mathrm{C}$ for $6 \mathrm{~min}$, and then centrifuged at $16,100 g_{\mathrm{n}}$ for $10 \mathrm{~min}$. Supernatant was removed, and pellet was washed with $500 \mu \mathrm{L}$ of cold $70 \%$ $\mathrm{EtOH}$ (by inverting the tubes carefully). Tubes were incubated at $-20^{\circ} \mathrm{C}$ for $5 \mathrm{~min}$., and then centrifuged at $16,100 g_{\mathrm{n}}$ for $5 \mathrm{~min}$. Supernatant was removed, and pellet was washed with $500 \mu \mathrm{L}$ of cold $90 \% \mathrm{EtOH}$ (repeating mixing, incubation, and centrifugation, as described before). EtOH was poured off and the pellet was dried at bench top for $\approx 30-45$ min (room temperature). The pellet DNA was resuspended in $50 \mu \mathrm{L}$ TE buffer (10 mu Tris- $\mathrm{HCl}, 0.1 \mathrm{~mm}$ EDTA) and $50 \mu \mathrm{L}$ of DNA grade water. DNA concentration was quantified in a spectrophotometer (UV10; Thermo Fisher Scientific). DNA concentration for all the samples was standardized to $20 \mathrm{ng} \cdot \mu \mathrm{L}^{-1}$.

\section{Genomic regions}

SSR MARKERS. A total of 41 SSR markers distributed across the peach genome $(\approx 11.3 \mathrm{cM}$ between markers $)$ were selected from the 'Texas' almond (Prunus dulcis) $\times$ 'Earlygold' peach $(\mathrm{T} \times \mathrm{E})$ reference map (Dirlewanger et al., 2004; Jung et al., 2008). Forward primers were modified using a $5^{\prime}$ fluorophore label 6-FAM (standard) (6-FAM) or HEX (5HEX) (Eurofins MWG Operon, Huntsville, AL) for multiplex product fragment analysis (Supplemental Table 1).

Polymerase chain reaction (PCR) products were amplified in a $16-\mu \mathrm{L}$ volume reaction containing $2 \mu \mathrm{L}$ of $20 \mathrm{ng} \cdot \mu \mathrm{L}^{-1} \mathrm{DNA}$ template, $2.25 \mu \mathrm{L} 10 \mathrm{X}$ ThermoPol Reaction Buffer $[10 \mathrm{~mm}$ $\mathrm{KCl}, 10 \mathrm{~mm}\left(\mathrm{NH}_{4}\right)_{2} \mathrm{SO}_{4}, 20 \mathrm{~mm}$ Tris- $\mathrm{HCl}, 2 \mathrm{~mm} \mathrm{MgSO}$, $0.1 \%$ Triton X-100, pH 8.8 at $25^{\circ} \mathrm{C}$ (New England Biolabs, Ipswich, MA)], $1 \mu \mathrm{L} 2.5 \mathrm{~mm}$ dNTPs, $0.2 \mu \mathrm{L}$ Taq DNA Polymerase (New England Biolabs), $6.55 \mu \mathrm{L}$ DNA grade water, and $4 \mu \mathrm{L} 5 \mu \mathrm{M}$ ( $2 \mu \mathrm{L}$ forward and $2 \mu \mathrm{L}$ reverse) primers. PCR parameters were 3 min at $94{ }^{\circ} \mathrm{C}$ followed by 40 cycles of 30 -s denaturing at $94{ }^{\circ} \mathrm{C}, 30 \mathrm{~s}$ at primer's specific annealing temperature $\left[\mathrm{Ta}\left({ }^{\circ} \mathrm{C}\right)\right]$ (Supplemental Table 1), and $1 \mathrm{~min}$ of elongation at $72{ }^{\circ} \mathrm{C}$, ending with $7 \mathrm{~min}$ at $72{ }^{\circ} \mathrm{C}$. PCR products were separated on $3 \%(\mathrm{~m} / \mathrm{v})$ agarose gel. Gels were stained with ethidium bromide and recorded using a digital gel documentation system. Gel images PCR amplification intensities were used to determine PCR dilution ratios for fragment analysis on a sequencer (ABI3730; Applied Biosystems ${ }^{\circledR}$, Grand Island, NY) at the Interdisciplinary Center for Biotechnology Research, University of Florida, Gainesville, FL. Fragment analysis of chromatographs was visualized using GeneMarker ${ }^{\circledR}$ (version 1.6; SoftGenetics, State College, PA) using 600 LIZ $^{\circledR}$ size standard (Applied Biosystems ${ }^{\circledR}$ ).

Chloroplast DNA. Eight cpDNA regions (with product amplification less than $1000 \mathrm{bp}$ ) were selected based on their high polymorphism rates or proportion of mutational events for Prunus. Primer sequences were obtained from Shaw et al. (2005, 2007) and Morris et al. (2008) (Supplemental Table 2). Chloroplast DNA targets were amplified in a $48-\mu \mathrm{L}$ volume reaction containing $6 \mu \mathrm{L}$ of $20 \mathrm{ng} \cdot \mu \mathrm{L}^{-1}$ DNA template, $6.75 \mu \mathrm{L}$ 
10X ThermoPol Reaction Buffer [10 mм KCl, $10 \mathrm{~mm}\left(\mathrm{NH}_{4}\right)_{2}$ $\mathrm{SO}_{4}, 20 \mathrm{~mm}$ Tris-HCl, $2 \mathrm{~mm} \mathrm{MgSO}$, 0.1\% Triton X-100, pH 8.8 at $25{ }^{\circ} \mathrm{C}$ ], $3 \mu \mathrm{L} 2.5 \mathrm{~mm}$ dNTPs, $0.6 \mu \mathrm{L}$ Taq DNA Polymerase, $19.65 \mu \mathrm{L}$ DNA grade water, and $12 \mu \mathrm{L} 5 \mu \mathrm{M}$ ( $6 \mu \mathrm{L}$ forward and $6 \mu \mathrm{L}$ reverse) primers. A universal cpDNA PCR procedure was used to simplify amplification as reported by Shaw et al. (2007). PCR cycling conditions were $5 \mathrm{~min}$ at $80{ }^{\circ} \mathrm{C}$ followed by 30 cycles of 1 -min denaturing at $95{ }^{\circ} \mathrm{C}$, followed by a ramp of $0.3{ }^{\circ} \mathrm{C} \cdot \mathrm{s}^{-1}$ to $65{ }^{\circ} \mathrm{C}$, and $4 \mathrm{~min}$ of elongation at $65^{\circ} \mathrm{C}$, ending with a final extension step of $5 \mathrm{~min}$ at $65{ }^{\circ} \mathrm{C}$. PCR products were confirmed on $3 \%(\mathrm{~m} / \mathrm{v})$ agarose gel. Gels were stained with ethidium bromide and recorded using a digital gel documentation system. Only cpDNA regions that were easily amplified for all the samples were used for further sequencing ( $n d h J$-trnF and trnT-trnL region primers did not amplify products for several species and therefore were not used for further analyses).

INTERNAL TRANSCRIBED SPACER. The use of ITS sequence data has proven of value to define species relationships within the genus Prunus (Bortiri et al., 2001, 2002, 2006; Lee and Wen, 2001). This region was amplified using the primers published by Wen and Zimmer (1996): C26A, 5'-GTT TCT TTT CCT CCG CT-3' and Nc18S10, 5'-AGG AGA AGT CGT AAC AAG-3'. PCR products were amplified in a $48-\mu \mathrm{L}$ volume reaction as previously described for $\mathrm{cpDNA}$ amplification. PCR cycling conditions were $3 \mathrm{~min}$ at $94{ }^{\circ} \mathrm{C}$ followed by 20 cycles of 1-min denaturing at $94{ }^{\circ} \mathrm{C}, 2 \mathrm{~min}$ at $50{ }^{\circ} \mathrm{C}$, and $2 \mathrm{~min}$ of elongation at $72^{\circ} \mathrm{C}$. PCR products were separated, stained, and visualized as previously described.

NuClear GENES. Single-copy genes associated with vernalization response (33), two major genes associated with tree architecture, and three genes expressing isozymes were included in this study. Hereafter, gene symbols will be used for these regions throughout the manuscript. The gene description corresponding to each gene region can be found in Supplemental Table 3. GenBank ${ }^{\circledR}$ annotated collection database of DNA sequences was used to find the Arabidopsis thaliana gene sequences and accessions corresponding to those genes (Benson et al., 2013). Arabidopsis gene sequences were blasted against the annotated peach genome v1.0 using BLASTTN option in Phytozome (Goodstein et al., 2012). BLAST results with the highest score and $E$ value were used to identify the best peach annotated transcript matching the arabidopsis genes. Gene ancestry for each peach transcript was analyzed in Phytozome to remove possible multiple copy gene targets. Peach gene sequences and their messenger RNA were aligned to identify exon-intron boundaries using MUSCLE, version 3.8 (Edgar, 2004). Specific primers for each region were created in Primer3Plus, version 2.3.5, web-interface (Untergasser et al., 2012) using consecutive exons as annealing sites to amplify across introns (unless otherwise specified). Similarly, an additional 14 candidate genes primer sets associated with axillary meristem formation and tree architecture in peach as reported by Carrillo-Mendoza (2012) were used (Supplemental Table 3).

PCR products were amplified in a $48-\mu \mathrm{L}$ volume reaction, as previously described for cpDNA and ITS regions amplification. PCR parameters were $3 \mathrm{~min}$ at $94^{\circ} \mathrm{C}$ followed by 40 cycles of 30 -s denaturing at $94{ }^{\circ} \mathrm{C}, 30 \mathrm{~s}$ at primer's specific Ta $\left({ }^{\circ} \mathrm{C}\right)$ (Supplemental Table 3), and $1 \mathrm{~min}$ of elongation at $72{ }^{\circ} \mathrm{C}$, ending with $7 \mathrm{~min}$ at $72{ }^{\circ} \mathrm{C}$. PCR products were separated, stained, and visualized as previously described.

\section{Sequencing}

PCR products with multiple bands during electrophoresis were separated, exised, and purified using QIAquick Gel Extraction Kit (Qiagen). Single band products were purified and direct-sequenced by Eurofins MWG Operon following company procedures. Geneious (R6.0.3; Biomatters, Auckland, New Zealand) was used to edit and assemble complementary DNA strands.

Assembled contigs of gene sequence regions were used to create internal primers that would improve sequence coverage, quality, and base-calling accuracy. The list of regions for which internal primers were created can be found in Supplemental Table 4. Two or more polymorphic sites were identified for CUC2, ELF6, FT-TSF, GI-FB, LFY, MAF4, and s6pdh. The polymorphisms were confirmed using the internal primers.

\section{Phylogenetic analyses}

Genetic variation and cluster analyses for SSR fingerprinting data were performed using Powermarker, version 3.25 (Liu and Muse, 2005) and GenAlEx, version 6.5 (Peakall and Smouse, $2006,2012)$. Genetic variation was characterized for each locus by the number of observed alleles (A), effective number of alleles (Ae), observed heterozygosity (Ho), expected heterozygosity $(\mathrm{He})$, Wright's fixation index $[F=(\mathrm{He}-\mathrm{Ho}) / \mathrm{He}=1-$ $(\mathrm{Ho} / \mathrm{He})]$, and polymorphism information content (PIC). Nei's genetic distance was calculated and used in neighbor joining (NJ) cluster analysis (Nei and Takezaki, 1983; Saitou and Nei, 1987).

Alignments of DNA sequences were performed using MUSCLE, version 3.8. Manual alignments were made in Geneious R6.0.3 to produce an alignment with the fewest number of changes. Alignments were visually checked for polymorphic sites that resulted in sequence slippage. Polymorphisms (indels and/or substitutions) were manually determined using reference sequences from other species with full sequence coverage (without slippage from forward and reverse sequencing). Alternative sequences or alleles were kept and used in further analyses. A consensus sequence was obtained for each genotype. All genotype sequences per region were realigned using MUSCLE, version 3.8. Haplotypes for each species were calculated based on aligned amplicons using DnaSP, version 5 (Librado and Rozas, 2009).

The number of nucleotide substitutions (Subst) and indels, collectively named potentially informative characters (PICS = Subst + Indels), between the ingroup species (American, Chickasaw, and Beach clades in Prunus section Prunocerasus), and between ingroup and outgroup species-phased haplotypes, were calculated using DnaSP, version 5. The proportion of observed mutational events or percent variability for each genomic region were calculated as reported by Shaw et al. $(2005,2007)$ by using the following equation: percent variability $=[($ Subst + Indels $) / L] \times 100$, where $L=$ the total aligned sequence length.

Phylogenetic analyses were performed by using the optimal criterion of maximum parsimony (MP) for diploid and phased haplotype sequences. Gaps (indels) were ignored and also considered as an additional binary character matrix, with $0=$ absence and $1=$ presence of the indel. MP trees were obtained by using PAUP*, version 4.0b10 (Swofford, 2002). A heuristic search for MPTs was conducted by using tree bisectionreconnection branch swapping and 1000 random sequence addition replicates. Bootstrap support (Felsenstein, 1985) was 


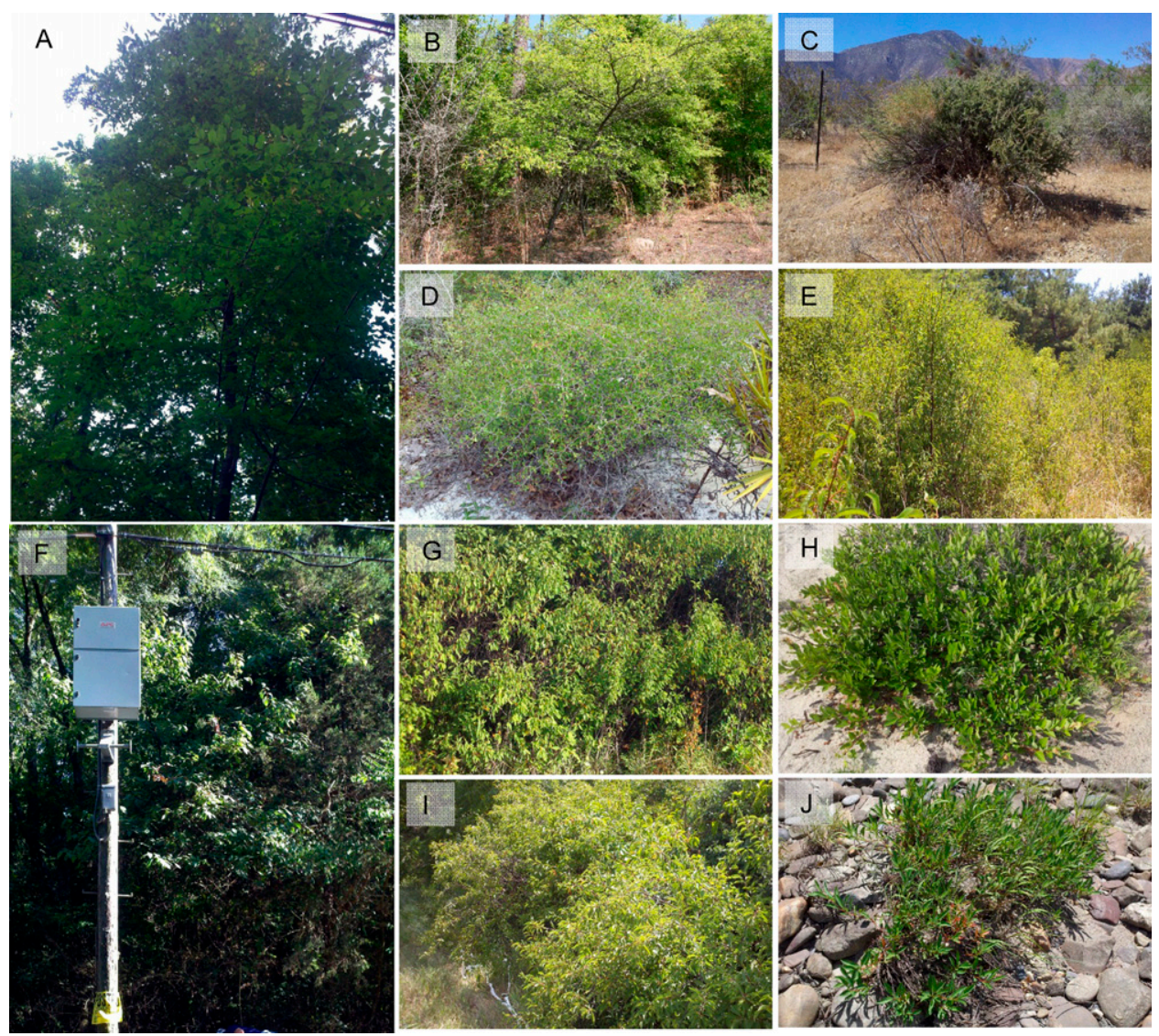

Fig. 1. Representatives of the North American plums (Prunus sp.), subgenus Prunus section Prunocerasus, and outgroup species: (A) Prunus americana, (B) Prunus umbellata, (C) Prunus fasciculata, (D) Prunus geniculata, (E) Prunus angustifolia, (F) Prunus mexicana, (G) Prunus hortulana, (H) Prunus maritima, (I) Prunus munsoniana, and (J) Prunus pumila.

estimated in 1000 replications of heuristic search and simple taxon addition with at least 100,000 rearrangements per replicate. Homoplasy was determined by calculating the consistency index and the retention index.

Model of sequence evolution for each region was determined using the Akaike information criterion [AIC (Akaike, 1974)] and Bayesian information criteria [BIC (Schwarz, 1978)] as implemented in the JModeltest, version 0.1.1 (Posada, 2008). A likelihood ratio test was calculated to choose the best model with the best $-\operatorname{lnL}$ score between the AIC and the BIC criteria. Heuristic searches for maximum likelihood trees (MLTs) were conducted using GARLI 2.0 (Zwickl, 2006) in the CIPRES Science Gateway (Miller et al., 2010). A nonparametric bootstrap was performed using two independent search replicates and 50 bootstrap repetitions. Best MLTs for each bootstrap repetitions and replicates were used to calculate a consensus tree with posterior probability values in Mesquite, version 2.73 (Maddison and Maddison, 2011).

Maximum likelihood analyses of individual and combined sequence data were performed using RAxML, version 7.1.0 (Stamatakis, 2006; Stamatakis et al., 2008). Analyses were calculated under a general-time-reversible model of nucleotide substitution and a gamma model of rate heterogeneity in the CIPRES Science Gateway. A halt bootstrapping, 1000 replicates, was performed. Best MLTs for each bootstrap repetition were used to calculate a consensus tree with posterior probability values in Mesquite, version 2.73.
Network analyses of haplotype and diploid sequence data were performed as described by Oatley et al. (2012). Networks of the phased haplotypes were estimated using TCS, version 1.21 (Clement et al., 2000). TCS analyses were performed treating gaps as missing data and as a fifth character state. Multilocus networks were built using SplitsTree, version 4.11.3 (Huson and Bryant, 2006) based on a distance matrix generated in POFAD, version 1.03 (Joly and Bruneau, 2006). Uncorrected $p$ distances from aligned sequences were calculated in PAUP*, version $4.0 \mathrm{~b} 10$ and were used as input in POFAD.

\section{Results}

\section{Simple sequence repeats}

The 41 SSR markers amplified 471 alleles in the 11 species representing outgroups and the major clades within subgenus Prunus section Prunocerasus (Fig. 1). An average of nine genotypes (out of 11 samples) were amplified per marker, with A of 10.70 , the Ae was 8.10 , Ho of $0.58, \mathrm{He}$ of $0.84, F$ of 0.31 , and PIC of 0.82 (Table 2). Thirty-four SSR markers successfully worked for all the species. Out of those, 15 SSR markers amplified a total of 11 genotypes in the 11 samples. The value of A varied from 17 for BPPCT025 to 3 for СРPCT015. ВРPCT023, ВРPCT025, and ВРPCT039 had the highest Ae of 13.44. СРРCT022 had the lowest Ae of 2.35. The value of Ho varied from 1.00 to 0.09 for СРPCT006 and EPDCU3117, respectively. BPPCT025, BPPCT026, and CPDCT025 had the highest He of 0.93 compared with the lowest He of 0.63 for CPPCT015. Wright's fixation index $(F)$ ranged from 0.87 for СРРCТ033 to -0.05 for ВРРCТ029. Six SSRs, ВРPCT025, ВРPCT026, ВРРCT039, СРDCT025, EPDC3832B, and EPDCU3392, had the highest PIC of 0.92 compared with CPPCT015 of 0.55 .

Nei's genetic distance (Nei and Takezaki, 1983) was calculated in combination with NJ (Saitou and Nei, 1987) cluster analysis for 41 SSR markers (Fig. 2A). Subgenus Prunus section Prunocerasus was monophyletic. Relationships within the North American plum species were not in agreement to those reported by Shaw and Small (2004). The outgroup species clustered together as expected. The Chickasaw Clade ( $P$. munsoniana and $P$. angustifolia) was recovered. The American Clade and Beach Clade were not observed.

Cluster analysis using BPРCT025, ВРPCT026, ВРРCТ039, CPDCT025, EPDC3832B, and EPDCU3392, the six SSR markers with the highest PIC values yielded somewhat consistent results (Fig. 2B). Outgroups clustered together, however, $P$. mexicana was found as a sister taxa to the outgroup. The Beach Clade ( $P$. geniculata and P. maritima) and Chickasaw 
Table 2. Summary statistics of 41 simple sequence repeat markers of a subset of 11 Prunus species.

\begin{tabular}{|c|c|c|c|c|c|c|c|c|}
\hline Marker & Species amplification $^{z}$ & Genotype no. & $\mathrm{A}^{\mathrm{y}}$ & $\mathrm{Ae}$ & Ho & $\mathrm{He}$ & $F$ & $\overline{\mathrm{PIC}}$ \\
\hline ВРРСТ006А & 10 & 10 & 7 & 5.56 & 0.60 & 0.82 & 0.27 & 0.80 \\
\hline ВРРСТ006В & 8 & 8 & 8 & 6.74 & 0.50 & 0.85 & 0.41 & 0.83 \\
\hline ВРРСТ008А & 10 & 8 & 8 & 5.41 & 0.50 & 0.82 & 0.39 & 0.79 \\
\hline ВРРСТ008В & 10 & 8 & 12 & 9.52 & 0.60 & 0.90 & 0.33 & 0.89 \\
\hline ВРРСТ013 & 11 & 10 & 9 & 6.54 & 0.73 & 0.85 & 0.14 & 0.83 \\
\hline ВРРСТ 014 & 11 & $11^{\mathrm{x}}$ & 14 & 11.52 & 0.55 & 0.91 & 0.40 & 0.91 \\
\hline ВРРСТ016 & 11 & 7 & 7 & 10.08 & 0.18 & 0.76 & 0.50 & 0.73 \\
\hline ВРРСТ017 & 11 & 10 & 13 & 2.85 & 0.45 & 0.90 & 0.02 & 0.89 \\
\hline ВРРСТ023 & 11 & 5 & 4 & 13.44 & 0.64 & 0.65 & 0.02 & 0.58 \\
\hline ВРРСТ025 & 11 & 11 & 17 & 13.44 & 0.91 & 0.93 & 0.21 & 0.92 \\
\hline ВРРСТ026 & 11 & 11 & 16 & 5.50 & 0.73 & 0.93 & 0.67 & 0.92 \\
\hline ВРРСТ027 & 11 & 8 & 9 & 9.68 & 0.27 & 0.82 & 0.39 & 0.80 \\
\hline ВРРСТ028 & 11 & 11 & 12 & 7.33 & 0.55 & 0.90 & 0.16 & 0.89 \\
\hline ВРРСТ029 & 11 & 10 & 10 & 4.57 & 0.73 & 0.86 & -0.05 & 0.85 \\
\hline ВРРСТ030 & 11 & 8 & 9 & 7.41 & 0.82 & 0.78 & 0.42 & 0.76 \\
\hline ВРРСТ038 & 10 & 8 & 12 & 12.74 & 0.50 & 0.87 & 0.21 & 0.85 \\
\hline ВРРСТ039 & 11 & 11 & 15 & 13.44 & 0.73 & 0.92 & 0.02 & 0.92 \\
\hline СРDCT023 & 10 & 7 & 7 & 8.64 & 0.20 & 0.74 & 0.07 & 0.71 \\
\hline СРDCT025 & 11 & 11 & 15 & 7.33 & 0.91 & 0.93 & 0.37 & 0.92 \\
\hline СРDCT027 & 11 & 11 & 13 & 10.52 & 0.82 & 0.88 & 0.10 & 0.87 \\
\hline СРDCT038 & 11 & 10 & 12 & 6.21 & 0.55 & 0.86 & 0.67 & 0.85 \\
\hline СРРСТ005 & 11 & 10 & 14 & 2.67 & 0.82 & 0.90 & 0.20 & 0.90 \\
\hline СРРСТ006 & 10 & 10 & 13 & 10.08 & 1.00 & 0.91 & 0.39 & 0.90 \\
\hline СРРСТ008 & 11 & 8 & 9 & 11.00 & 0.27 & 0.84 & 0.30 & 0.82 \\
\hline СРРСТ015 & 2 & 2 & 3 & 9.68 & 0.50 & 0.63 & 0.39 & 0.55 \\
\hline СРРСТ022 & 11 & 11 & 13 & 2.35 & 0.55 & 0.90 & 0.05 & 0.89 \\
\hline СРРСТ026 & 11 & 11 & 14 & 8.07 & 0.64 & 0.91 & 0.17 & 0.90 \\
\hline СРРСТ029 & 11 & 6 & 6 & 12.74 & 0.27 & 0.68 & 0.11 & 0.64 \\
\hline СРРСТ033 & 11 & 11 & 13 & 3.06 & 0.55 & 0.90 & 0.87 & 0.89 \\
\hline СРSCT004 & 11 & 7 & 8 & 8.00 & 0.55 & 0.57 & -0.03 & 0.56 \\
\hline СРSCT008 & 3 & 3 & 5 & 6.21 & 0.67 & 0.78 & 0.24 & 0.74 \\
\hline CPSCT034 & 11 & 10 & 12 & 12.10 & 0.55 & 0.89 & 0.31 & 0.88 \\
\hline EPDC3832A & 11 & 10 & 12 & 7.81 & 0.73 & 0.88 & 0.27 & 0.86 \\
\hline EPDC3832B & 11 & 11 & 16 & 4.48 & 0.82 & 0.92 & 0.77 & 0.92 \\
\hline EPDCU3117 & 11 & 5 & 5 & 8.64 & 0.09 & 0.67 & 0.59 & 0.63 \\
\hline EPDCU3392 & 11 & 11 & 15 & 3.92 & 0.82 & 0.92 & 0.60 & 0.92 \\
\hline EPPISF002 & 10 & 9 & 10 & 10.52 & 0.90 & 0.88 & 0.20 & 0.86 \\
\hline EPPISF032 & 11 & 10 & 10 & 5.56 & 0.64 & 0.84 & 0.27 & 0.82 \\
\hline PMS2 & 11 & 11 & 15 & 6.74 & 0.64 & 0.92 & 0.41 & 0.91 \\
\hline UDP96-001 & 11 & 11 & 10 & 5.41 & 0.64 & 0.87 & 0.39 & 0.86 \\
\hline UDP96-003 & 11 & 8 & 8 & 9.52 & 0.18 & 0.78 & 0.33 & 0.76 \\
\hline UDP96-019 & 11 & 9 & 12 & 6.54 & 0.36 & 0.88 & 0.14 & 0.87 \\
\hline UDP98-025 & 11 & 7 & 5 & 11.52 & 0.30 & 0.75 & 0.40 & 0.70 \\
\hline UDP98-412 & 11 & 11 & 14 & 10.08 & 0.73 & 0.90 & 0.50 & 0.90 \\
\hline Average & - & 9 & 10.70 & 8.10 & 0.58 & 0.84 & 0.31 & 0.82 \\
\hline
\end{tabular}

${ }^{\mathrm{z}}$ Number of species where successful amplification occurred.

${ }^{\mathrm{y}} \mathrm{A}=$ number of observed alleles, $\mathrm{Ae}=$ effective number of alleles, Ho = observed heterozygosity, $\mathrm{He}=$ expected heterozygosity, $F=$ Wright's fixation index $[\mathrm{F}=(\mathrm{He}-\mathrm{Ho}) / \mathrm{He}=1-(\mathrm{Ho} / \mathrm{He})], \mathrm{PIC}=$ polymorphism information content.

${ }^{\mathrm{x}}$ Numbers in bold represent highest and lowest values for each variable.

Clade (P. angustifolia and P. munsoniana) were recovered. Prunus americana and P. umbellata were monophyletic. Prunus hortulana was recovered as sister species to all the section Prunocerasus Clade.

\section{Chloroplast DNA}

Analysis of the seven cpDNA regions indicated that the $n d h J-t r n F$ region did not amplify consistently and was removed for further analyses (Supplemental Table 2). Phased haplotype sequences were generated for the 11 species, for a total of $3584 \mathrm{bp}$ containing 130 PICS and an average polymorphism rate of $3.68 \%$ (Table 3 ). The most informative sites were observed in 3'trn $\mathrm{V}$-ndhC and $t r n H-$ psbA cpDNA regions for section Prunocerasus with $7.23 \%$ and $3.28 \%$ variability, respectively. Similar results were identified among the outgroup species with $3.17 \%$ and $1.49 \%$ variability, respectively (Fig. 3; Table 3). Both regions yielded good species delimitations within section Prunocerasus and outgroup species in MP and ML analyses, similar to those reported by Shaw and Small (2004) (Supplemental Figs. 1 and 4; Supplemental Tables 6 and 7). MP analyses with gaps as an additional character helped identifying additional species relationships in comparison with MP analyses without gaps. Similarly, MP and ML analyses were consistent in all and across the cpDNA regions for sequence data (Supplemental Figs. 1-6).

Phylogenetic analyses of trnL$\operatorname{trn} F$, trnL intron and trnQ-5'rps 16 cpDNA regions recovered internal relationships for section Prunocerasus (Supplemental Figs. 2 and 3, respectively). Section Prunocerasus was found monophyletic in MP and ML analyses with diploid or with haplotype number prefix data. In addition, $P$. americana and $P$. mexicana (American Clade) were found clustered together for all cpDNA regions (except $a t p B-r b c L$ ). Prunus geniculata was found near the base of section Prunocerasus for trnL$\operatorname{trnF}$ and $\operatorname{trnL}$ intron, trnQ-5' rps 16 , and $\operatorname{trn} H-p s b A$. Other cpDNA regions were consistent with previous results. Phased haplotype MP analyses found that $P$. angustifolia, $P$. umbellata, and $P$. munsoniana shared the same cpDNA haplotype for $\operatorname{trn} L-\operatorname{trn} F$ and $\operatorname{trn} L$ introns, $\operatorname{trn} Q$ 5'rps16, trnH-psbA, ndhF-rpL32, and $a t p B-r b c L$. The results are congruent with the Chickasaw Clade as described by Shaw and Small (2004).

Combined cpDNA regions phylogenetic analyses using MP and ML criteria found section Prunocerasus as a monophyletic clade (Fig. 4). Prunus americana and P. mexicana were found clustered together in all analyses. Prunus geniculata was identified as a basal sister species to $P$. americana and $P$. mexicana. Similarly, $P$. hortulana, $P$. munsoniana, $P$. maritima, 

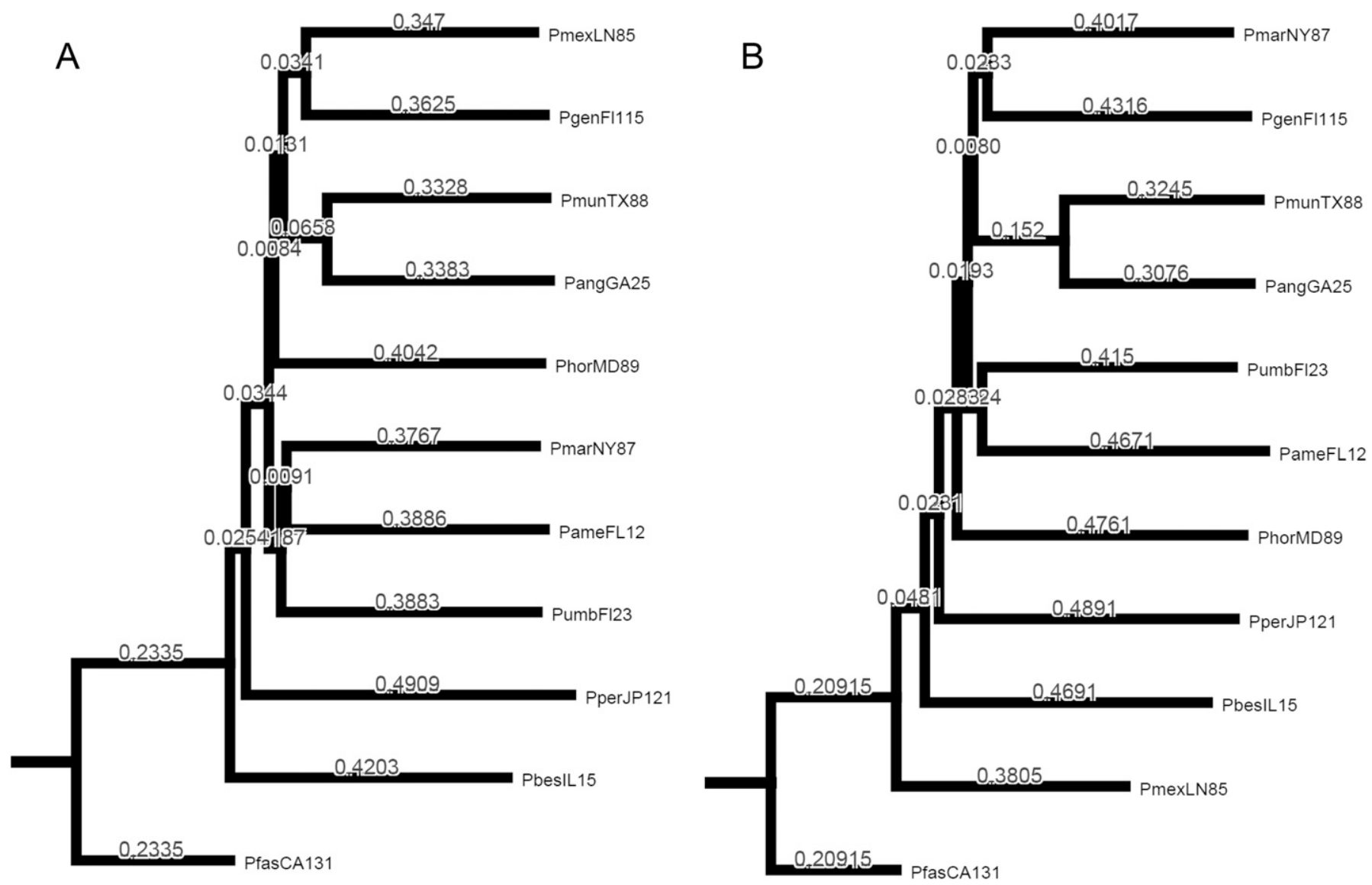

Fig. 2. Neighbor joining cluster analysis based on Nei's genetic distance (Nei and Takezaki, 1983) for 11 Prunus species using (A) 41 simple sequence repeat (SSR) markers and (B) six SSR markers with high polymorphism information content values. Cladogram rooted with Prunus fasciculata (PfasCA131) at the base. Other species in the cladogram are: P. americana (PameFL12), P. angustifolia (PangGA25), P. geniculata (PgenFL115), P. hortulana (PhorMD89), P. maritima (PmarNY87), P. mexicana (PmexLN85), P. munsoniana (PmunTX88), P. persica (PperJP121), P. pumila (PbesIL15), and P. umbellata (PumbFL23).

P. umbellata, and P. angustifolia were clustered together. MP and MLTs were consistent across regions. The nonstandarized multilocus combined cpDNA sequence data network (Fig. 5) recovered similar species relationships to those produced by MP and ML analyses. Additional sequence data information and models of sequence evolution are available in Supplemental Tables 6, 7, 8, and 10 .

\section{Internal transcribed spacer}

A target of $610 \mathrm{bp}$ of alignable haplotype sequence containing 66 PICS and an average variability of $10.82 \%$ was obtained (Table 4). The sequence variability of section Prunocerasus was $2.30 \%$ compared with the outgroup variability of $8.52 \%$ (Fig. 3). Section Prunocerasus was found monophyletic for both, MP and ML, analyses (Supplemental Fig. 7; Supplemental Tables 6 and 7). When the sequence data were analyzed without the insertion of gaps, $P$. munsoniana and P. americana were clustered together in MP. However, these species relationship was lost when the gaps were used as an additional character in MP analyses. Other lower level species relationships among the North American plums were not recovered. The results obtained were consistent across diploid and phased haplotype sequence data analyses. Additional sequence data information and models of sequence evolution are presented in Supplemental Tables 6, 7, 9, and 11.

\section{Nuclear genes}

IsozyMEs. Three isozyme genes, $P G I, P G D H$, and $s 6 p d h$, were sequenced in the 11 core species and two peach haploids. Analysis of the $1405 \mathrm{bp}$ of alignable sequence detected 117 PICS with a variability of $8.77 \%$ (Table 4). The PGI primers used did not amplify the $P$. americana PGI sequence. Readable sequences were obtained for all other species. The highest level of polymorphism within section Prunocerasus and between the outgroup taxa was obtained for PGI, followed by s6pdh and PGDH (Table 4). The sequence polymorphism rate for PGI within section Prunocerasus was $7.69 \%$ compared with $4.93 \%$ and $3.56 \%$ for s6pdh and PGDH, respectively. Similar trends of $4.01 \%, 3.18 \%$, and $2.94 \%$ polymorphism rate, respectively, were observed when the outgroup species $(P$. fasciculata, $P$. persica, and P. pumila) were removed (Fig. 3).

Section Prunocerasus was recovered as a monophyletic clade with MP and ML analyses using either diploid or haplotype datasets for PGI and PGDH genes. MP analyses using gaps as an additional source of information for the enzyme sequences did not provide additional resolution of species relationships (Supplemental Figs. 8-10; Supplemental Tables 6 and 7).

Prunus munsoniana and $P$. angustifolia consistently clustered together in all diploid and phased haplotype sequence data analyses with MP and ML for all isozyme genes. Both species integrated into the Chickasaw Clade, as previously described. Other previously known species relationships, such as the 
Table 3. Summary statistics of cpDNA (chloroplast DNA) regions sequenced for 11 Prunus species.

\begin{tabular}{|c|c|c|c|c|c|c|c|c|c|c|c|c|c|c|}
\hline \multirow{3}{*}{$\frac{\text { cpDNA region }}{\text { Aligned } L(\mathrm{bp})^{\mathrm{y}}}$} & \multicolumn{2}{|c|}{$3^{\prime} \operatorname{trn} V-n d h C$} & \multicolumn{2}{|c|}{$\operatorname{trn} L-\operatorname{trn} F^{\mathrm{z}}$} & \multicolumn{2}{|c|}{ trnL intron } & \multicolumn{2}{|c|}{$\operatorname{trn} Q-5^{\prime} r p s 16$} & \multicolumn{2}{|c|}{$\operatorname{trnH}-p s b A$} & \multicolumn{2}{|c|}{ ndhF-rpl32 } & \multicolumn{2}{|c|}{$a t p B-r b c L$} \\
\hline & \multicolumn{2}{|c|}{567} & \multicolumn{2}{|c|}{472} & \multicolumn{2}{|c|}{364} & \multicolumn{2}{|c|}{479} & \multicolumn{2}{|c|}{335} & \multicolumn{2}{|c|}{598} & \multicolumn{2}{|c|}{769} \\
\hline & Indels & Subst & Indels & Subst & Indels & Subst & Indels & Subst & Indels & Subst & Indels & Subst & Indels & Subst \\
\hline American $^{\mathrm{x}}$ & 17 & 19 & 0 & 0 & 0 & 2 & 4 & 3 & 8 & 2 & 1 & 0 & 0 & 0 \\
\hline Chickasaw $^{\mathrm{x}}$ & 0 & 0 & 0 & 0 & 0 & 0 & 1 & 0 & 5 & 0 & 0 & 0 & 0 & 0 \\
\hline Prunocerasus ${ }^{\mathrm{x}}$ & 25 & 16 & 0 & 0 & 0 & 3 & 5 & 4 & 9 & 2 & 2 & 0 & 0 & 1 \\
\hline $\mathrm{PICS}^{\mathrm{w}}$ & \multicolumn{2}{|c|}{41} & \multicolumn{2}{|l|}{0} & \multicolumn{2}{|l|}{3} & \multicolumn{2}{|c|}{9} & \multicolumn{2}{|c|}{11} & \multicolumn{2}{|c|}{2} & \multicolumn{2}{|c|}{1} \\
\hline Outgroup $^{\mathrm{u}}$ & 9 & 9 & 0 & 3 & 1 & 5 & 5 & 5 & 2 & 3 & 3 & 6 & 3 & 9 \\
\hline PICS & \multicolumn{2}{|c|}{18} & \multicolumn{2}{|c|}{3} & \multicolumn{2}{|c|}{6} & \multicolumn{2}{|c|}{10} & \multicolumn{2}{|c|}{5} & C & & 12 & \\
\hline Percent variability & & & 0. & & 1. & & & & & 49 & & & & 56 \\
\hline Total PICS & 59 & & 3 & & 9 & & 1 & & 16 & & 11 & & 13 & \\
\hline Normalized PICS & & & 0 . & & 0. & & & & & & & & & 67 \\
\hline
\end{tabular}

${ }_{\mathrm{z}}$ trnL-trnF and $\operatorname{trnL}$ introns were sequenced together as they primers amplifying both regions.

${ }^{\mathrm{y}}$ Aligned $L=$ total aligned sequence length.

${ }^{\mathrm{x}}$ American Clade $=$ Prunus americana, Prunus hortulana , and Prunus mexicana; Beach Clade = Prunus geniculata and Prunus maritima; Chickasaw Clade $=$ Prunus angustifolia, Prunus munsoniana, and Prunus umbellata $;$ Prunocerasus $=$ American Clade + Beach Clade + Chickasaw Clade.

${ }^{w}$ Potentially informative characters $($ PICS $)=$ Indels + Subst. Indels $=$ number of insertion/deletion events; subst $=$ substitutions .

${ }^{\mathrm{v}}[($ Subst + Indels $) / L] \times 100$, where $L=$ the total aligned sequence length.

"Outgroup = Prunus fasciculata, Prunus persica, and Prunus pumila.

American Clade and Beach Clade, were not recovered. MP and ML analyses of PGI, diploid and phased haplotype sequence data, recovered $P$. geniculata and $P$. hortulana clustered together at the base of the section Prunocerasus Clade (Supplemental Fig. 9). ML analysis of PGI haplotypes recovered the Chickasaw Clade (including $P$. umbellata). Analysis of the s6pdh sequence data produced a polytomy consisting of $P$. americana, $P$. geniculata, $P$. persica, $P$. pumila, and $P$. fasciculata (Supplemental Fig. 10).

MP and ML haplotype analyses for PGDH revealed that $P$. geniculata, $P$. angustifolia, and $P$. munsoniana shared a common haplotype. In addition, $P$. geniculata was located basal to the section Prunocerasus species in a polytomy with $P$. persica and P. pumila (PbesIL15) (Supplemental Fig. 8).

MP and ML analyses of the combined diploid isozyme data recovered section Prunocerasus as a monophyletic group. Prunus angustifolia and P. munsoniana were clustered together in all analyses (Chickasaw Clade). MP analyses (with and without gaps) did not provide additional resolution of species relationships. However, ML analyses of combined data recovered the Chickasaw Clade as a monophyletic group ( $P$. umbellata, $P$. angustifolia, and $P$. munsoniana). The American Clade (P. americana and P. mexicana) was found clustered to $P$. maritima as a sister species. Prunus hortulana and $P$. geniculata were identified as basal to species within section Prunocerasus (Supplemental Fig. 63).

The nonstandarized multilocus (phased haplotype) combined isozymes sequence data network (Supplemental Fig. 64) recovered similar species relationships to those produced by MP and ML analyses. The Chickasaw Clade and American Clade were recovered. Prunus maritima was identified clustered to P. umbellata. Prunus pumila was found basal to all North American plums similar to the other outgroup species. Additional sequence data information and models of sequence evolution are available in Supplemental Tables 5, $6,7,9$, and 11 .
Tree architecture. A total of 16 axillary meristem formation and tree architecture genes [AXR1, BRC1, BRC2, CUC1, CUC2, CUC3, LAS, MAX1, MAX2, MAX3, MAX4, PIN, RAX1, RAX2-RAX3, REV, and SPS (Supplemental Table 3)] were sequenced in the 11 core species and 2 peach haploids. CUC1 and MAX1 amplified two targets in all the species studied. Both amplicons were purified, sequenced independently, and identified by A and B suffixes (i.e., CUC1A and CUC1B). Prunus angustifolia did not have a product being amplified corresponding to CUC1A (only product amplified for CUC1B).

A total of $6664 \mathrm{bp}$ of sequence containing 426 PICS and having an average polymorphism rate of $6.39 \%$ was obtained (Table 4). Two or more indel polymorphisms were detected in the CUC2 amplicon. Additional internal primers were designed to allow the generation of unambiguous sequence data as specified in Supplemental Table 4. Primers designed for all the target sequences yielded product for all species. However, we were not able to completely sequence the BRC2 amplicon from $P$. americana. The highest polymorphisms rate within section Prunocerasus and between outgroup were obtained for MAX4, MAX1, CUC2, MAX3, and AXR1 (Table 4). Percent variability within section Prunocerasus was 5.02\%, 5.53\% (3.36\% for MAX1A and 2.48\% for MAX1B), 3.12\%, 2.89\%, and 2.28\%, respectively (Fig. 3). A similar trend was observed for these genes polymorphism rate when the outgroup species was used for the analyses. MP and ML analyses of diploid and haplotype datasets for AXR1, BRC1 (only for MP), BRC2, CUC2, CUC3, LAS, MAX1 [MAX1A and MAX1B (only for MP)], MAX2, MAX3, MAX4, RAX1, RAX2-RAX3, REV, and SPS recovered section Prunocerasus as a monophyletic clade (Supplemental Figs. 1128; Supplemental Tables 6 and 7). Additional results per gene region can be found in Supplemental Information.

MP and ML analyses of all the tree architecture genes diploid sequences recovered section Prunocerasus as a monophyletic group. Prunus angustifolia and P. munsoniana were 

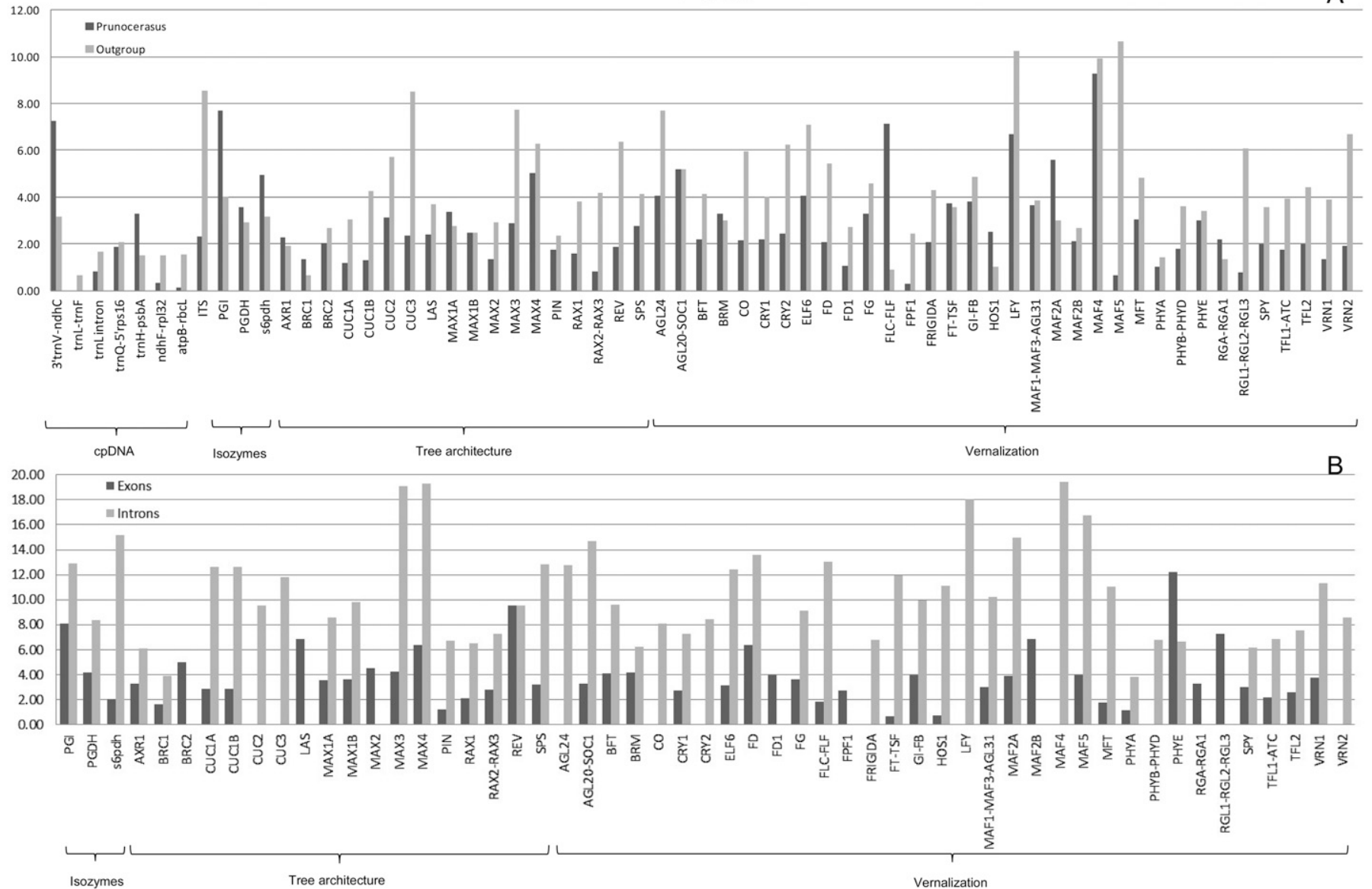

Fig. 3. Sequence variability $[($ substitutions + indels $) / L] \times 100$, where $L=$ the total aligned sequence length, comparisons for (A) section Prunocerasus vs. outgroup species and (B) exons vs. introns gene regions.

clustered together in all analyses (Chickasaw Clade). Similarly, $P$. americana and P. mexicana (American Clade), and $P$. maritima and $P$. umbellata, formed monophyletic clades, respectively. MP analyses (with gaps) yielded two major groups: 1) American Clade (P. mexicana, $P$. americana, and P. hortulana) and $P$. geniculata and 2) Chickasaw Clade ( $P$. umbellata, $P$. angustifolia, and $P$. munsoniana) and $P$. maritima. ML analyses of all sequence data recovered similar species groups to MP analysis with gaps. Prunus pumila was identified as a sister species to section Prunocerasus (Supplemental Fig. 65).

The nonstandarized multilocus (phased haplotype) combined tree architecture genes sequence data network (Supplemental Fig. 66) recovered similar species relationships to those produced by MP (with gaps) and ML analyses. The Chickasaw Clade and American Clade were recovered. Prunus maritima was identified clustered to $P$. umbellata (similar to the isozymecombined data analyses, Supplemental Fig. 64). Additional sequence data information and models of sequence evolution are presented in Supplemental Tables 5, 6, 7, 9, and 11.

Vernalization. A total of 33 vernalization-related genes [AGL20-SOC1, AGL24, BFT, BRM, CO, CRY1, CRY2, ELF6, FD, FD1, FG, FLC-FLF, FPF1, FRIGIDA, FT-TSF, GI-FB, HOS1, LFY, MAF1-MAF3-AGL31, MAF2, MAF4, MAF5, MFT, PHYA, PHYB-PHYD, PHYE, RGA-RGA1, RGL1-RGL2-RGL3, SPY, TFL1-ATC, TFL2, VRN1, and VRN2 (Supplemental Table 3)] were sequenced in the 11 core species and 2 peach haploids. MAF2 yielded duplicate products for all species. Duplicate bands were excised and sequenced as described in the Materials and Methods section. Duplicate products were designed using A and B suffixes (i.e., MAF2A and MAF2B).

The AGL20-SOC1 amplicons for $P$. americana and $P$. munsoniana could not be sequenced using the available primers and were removed from the dataset. Prunus pumila, $P$. americana, and P. fasciculata could not be sequenced for FLC-FLF. FRIGIDA sequences were difficult to assemble. Prunus umbellata, $P$. angustifolia, $P$. mexicana, $P$. hortulana, and $P$. munsoniana were not able to be sequenced for FRIGIDA. Similarly, $P$. geniculata and $P$. pumila were not sequenced for MAF2A, and P. angustifolia and haploids were not sequenced for MAF2B. The problems with the MAF2 amplicon were due to absence/presence of duplicate amplicon in some species.

A total of $15,646 \mathrm{bp}$ of alignable sequence (1206 PICS, polymorphism rate of $7.71 \%$ ) was obtained (Table 4). Two or more indels were identified for the ELF6, FT-TSF, GI-FB, LFY, and MAF4 amplicons in one or more species. These amplicons were resequenced for all the samples using specific internal primers as indicated in Supplemental Table 4.

The highest polymorphism rates were observed in MAF4, FLC-FLF, LFY, MAF2A, AGL20-SOC1, ELF6, and AGL24 (Table 4). Percent variability within section Prunocerasus was 

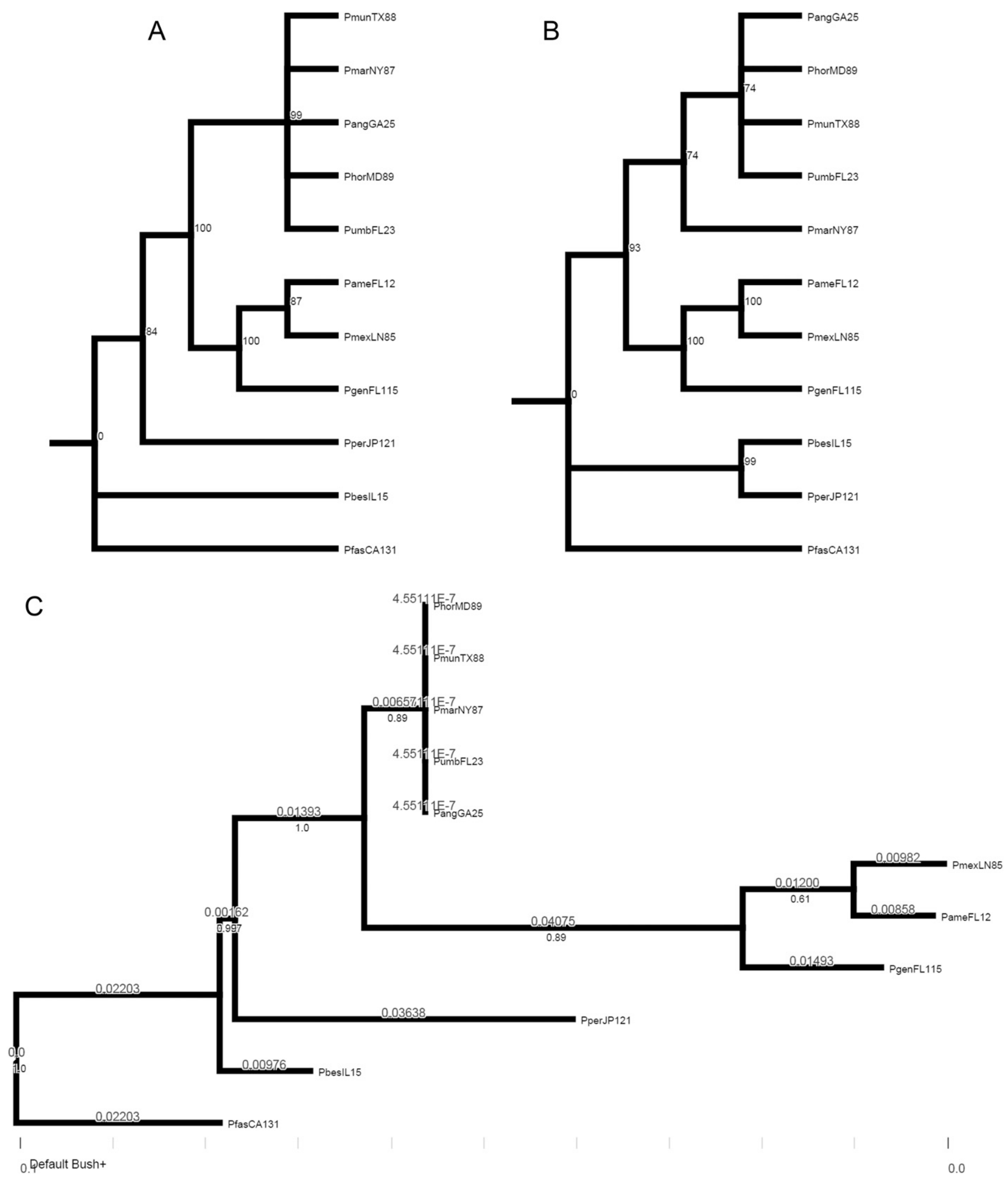

Fig. 4. Phylogenetic analyses of combined chloroplast DNA sequence data (3584 bp). Majority rule consensus trees using (A) maximum parsimony [75 trees, 126 steps, consistency index $(\mathrm{CI})=0.929$, retention index $(\mathrm{RI})=0.890$, rescaled consistency index $(\mathrm{RC})=0.827]$ and $(\mathrm{B})$ maximum parsimony (including gaps) $(13$ trees, 623 steps, $\mathrm{CI}=0.918, \mathrm{RI}=0.809, \mathrm{RC}=0.743)$ analyses. Bootstrap values greater than $50 \%$ are described above the branches. $(\mathbf{C})$ Maximum likelihood tree $(-\operatorname{lnL}=5414.74)$. Branch lengths are described above the branches and posterior probability values below the branches. Trees are rooted with Prunus fasciculata. Other species in the cladogram are: P. americana (PameFL12), P. angustifolia (PangGA25), P. geniculata (PgenFL115), P. hortulana (PhorMD89), P. maritima (PmarNY87), P. mexicana (PmexLN85), P. munsoniana (PmunTX88), P. persica (PperJP121), P. pumila (PbesIL15), and P. umbellata (PumbFL23). 
A

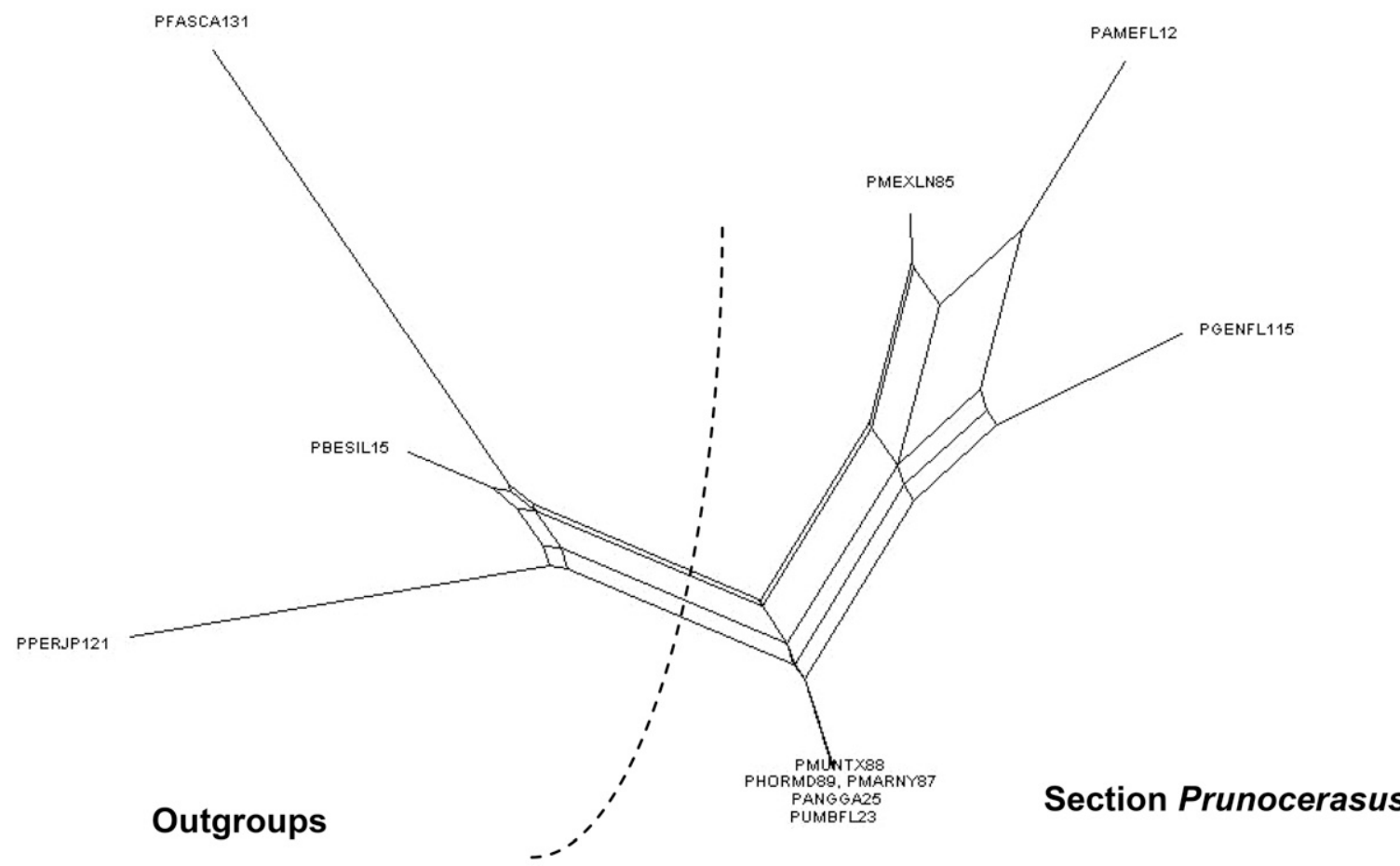

B

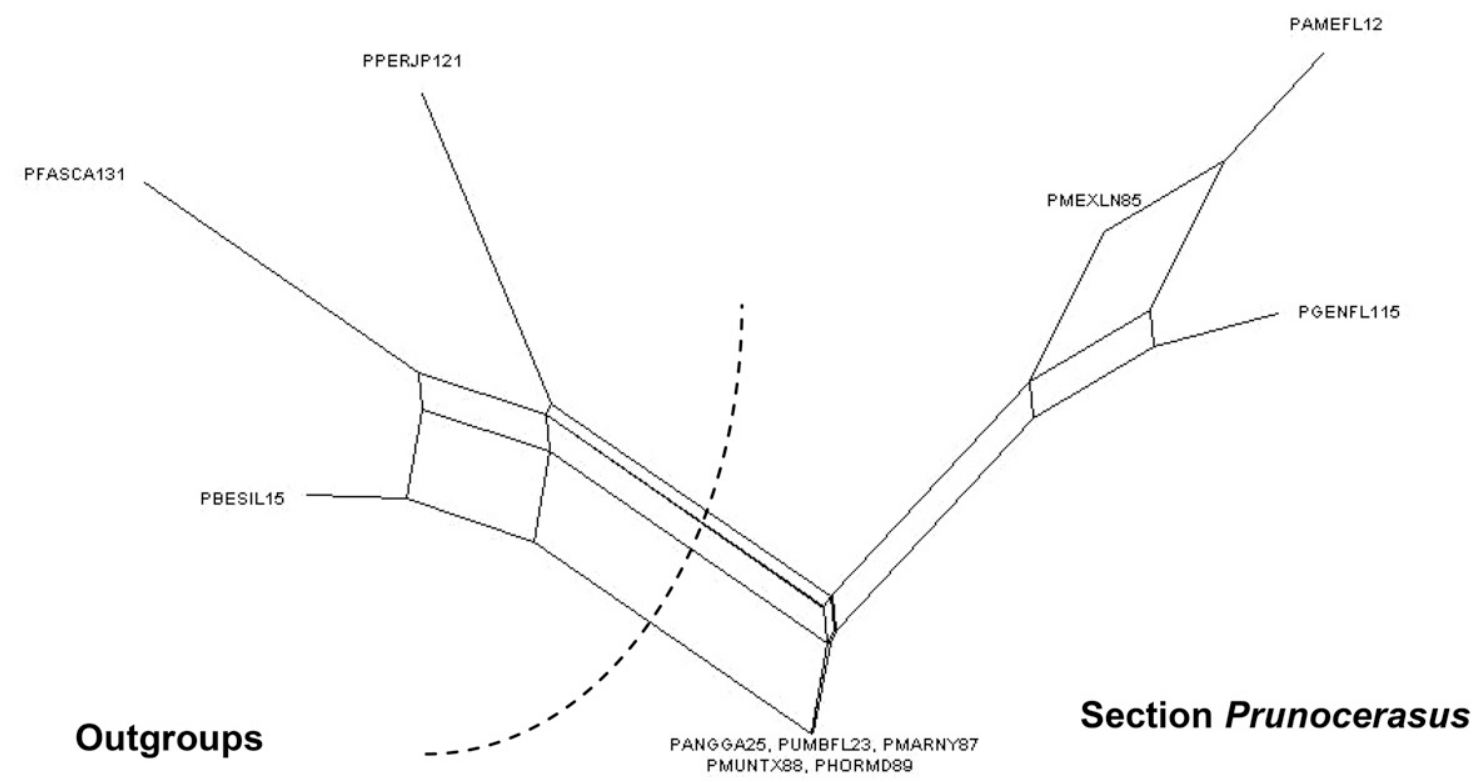

Fig. 5. A nonstandarized multilocus combined chloroplast DNA sequence data network using: (A) gaps as missing characters and (B) gaps as fifth characters. Prunus species in the cladogram are: P. americana (PameFL12), P. angustifolia (PangGA25), P. fasciculata (PfasCA131), P. geniculata (PgenFL115), P. hortulana (PhorMD89), P. maritima (PmarNY87), P. mexicana (PmexLN85), P. munsoniana (PmunTX88), P. persica (PperJP121), P. pumila (PbesIL15), and P. umbellata (PumbFL23). 


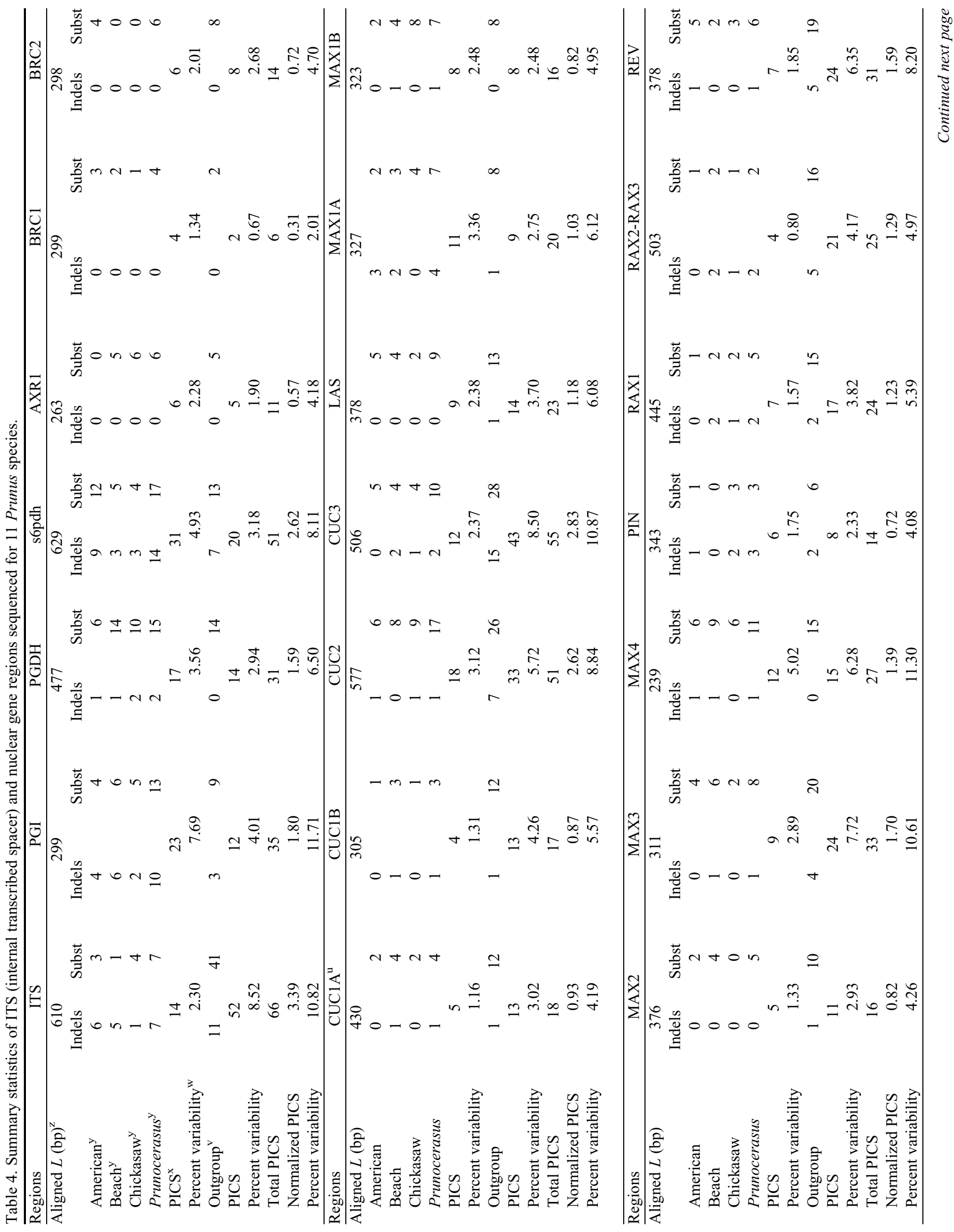




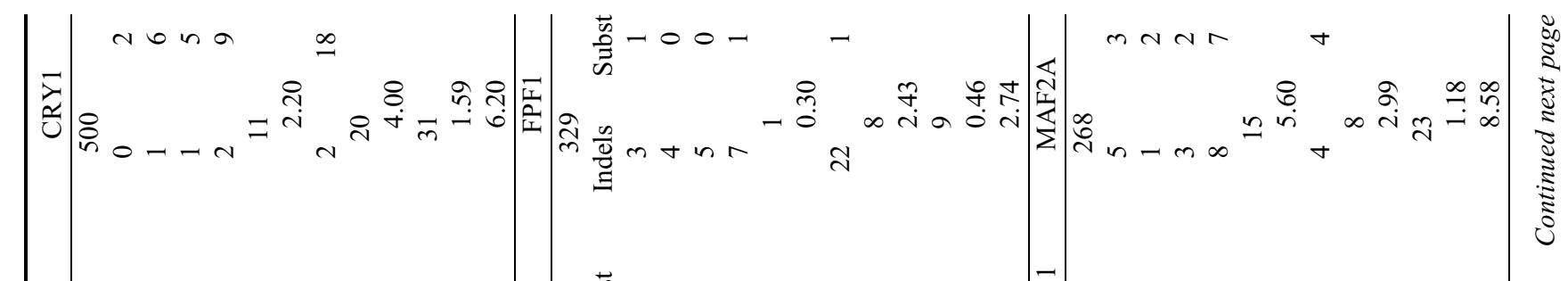

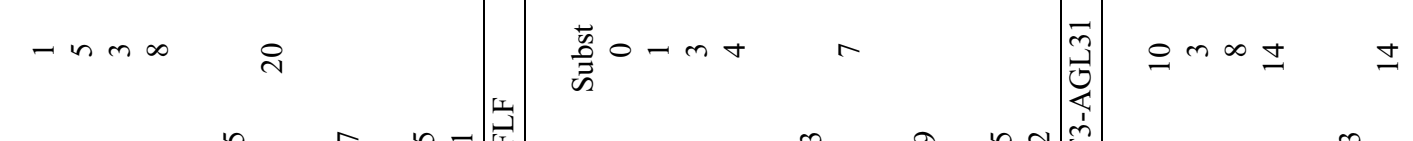

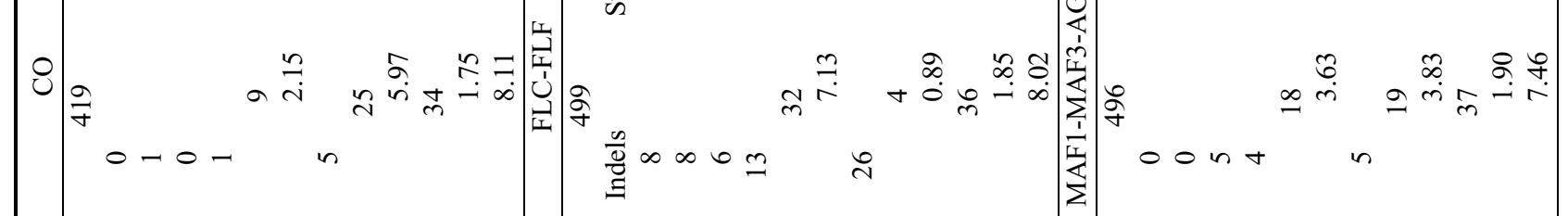

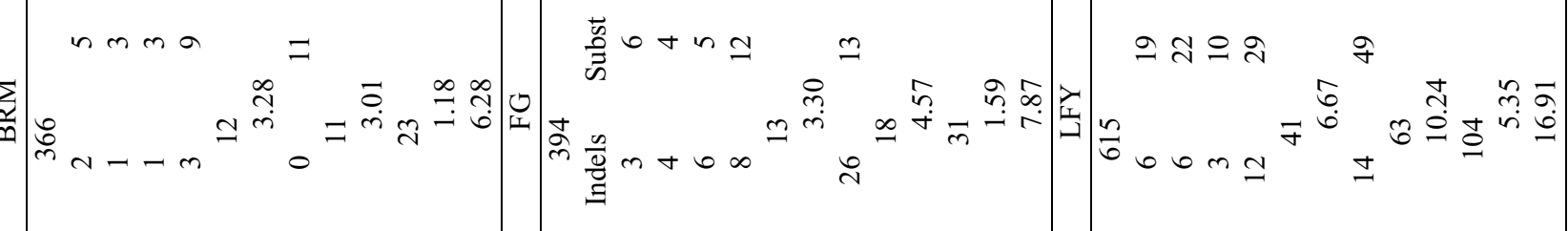

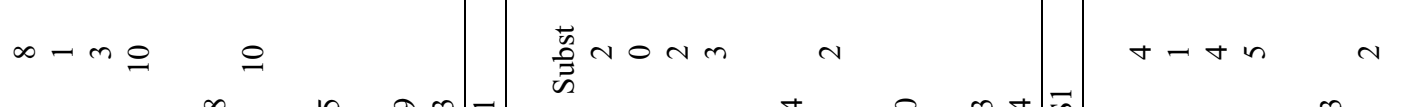

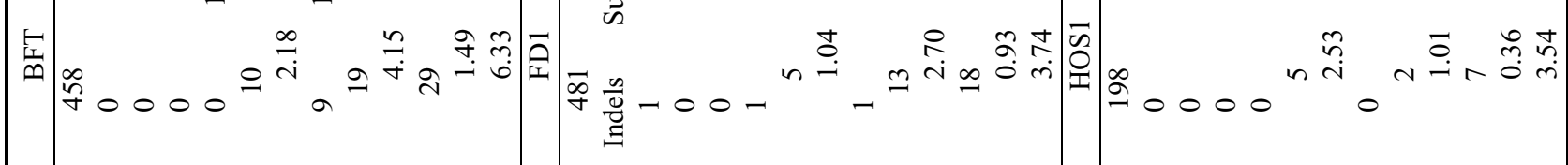

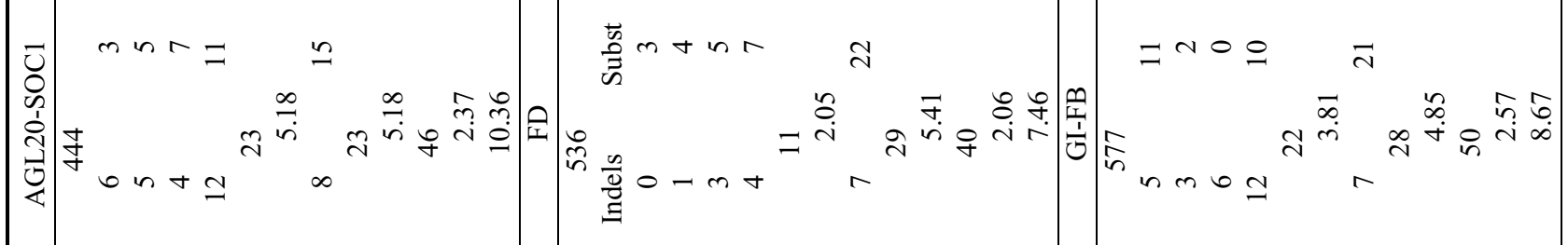

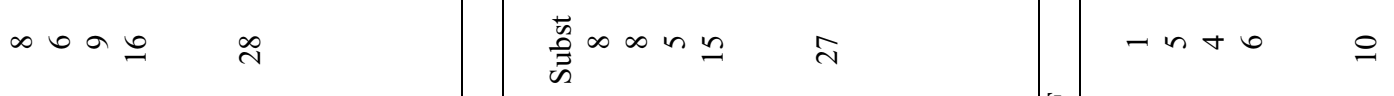

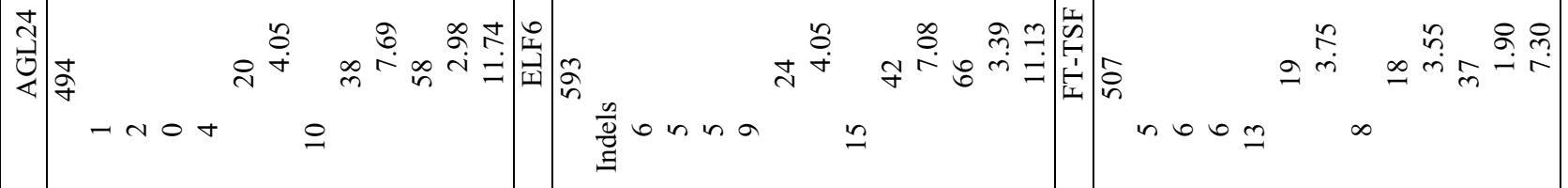

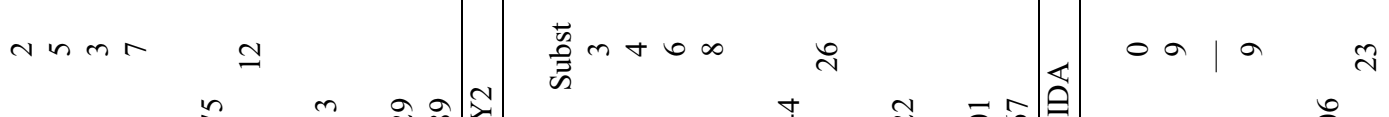

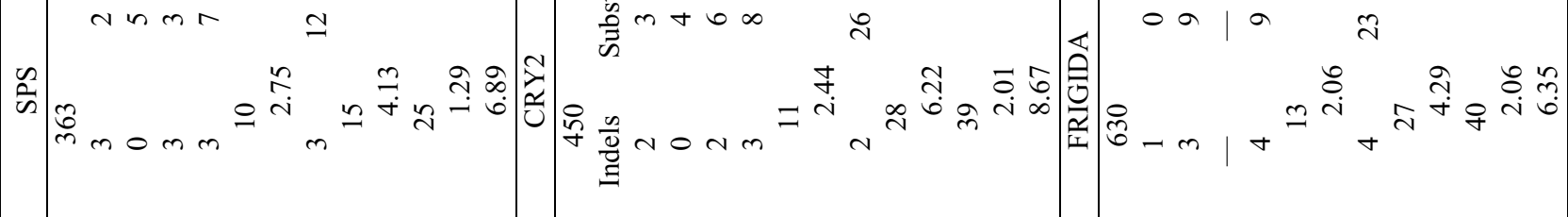

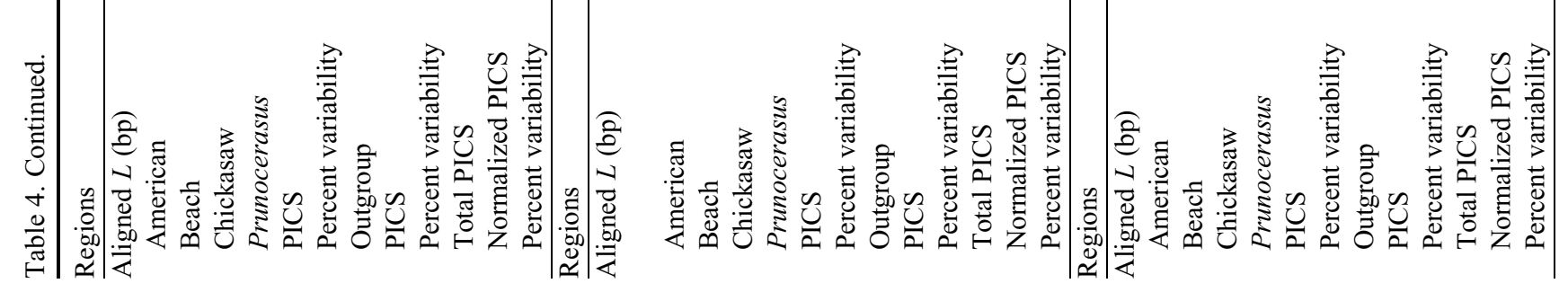




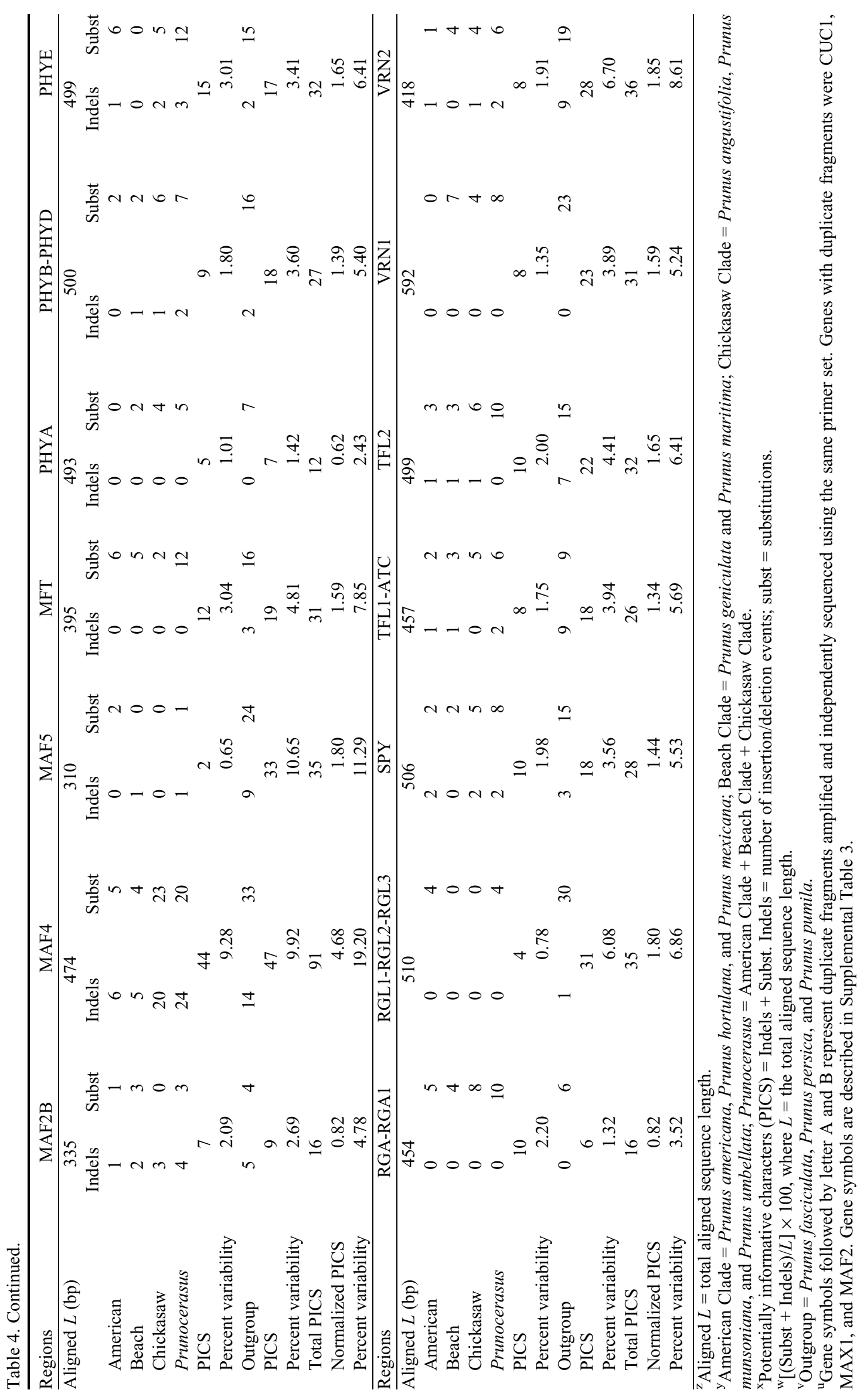


$9.28 \%, 7.13 \%, 6.67 \%, 5.60 \%, 5.18 \%, 4.05 \%$, and $4.05 \%$, respectively (Fig. 3). Percent variability for the outgroup species did not show a similar trend. Some genes had higher polymorphic rates among outgroup species. Section Prunocerasus was monophyletic in MP and ML analyses using diploid and haplotype datasets for all gene regions except FPF1, MAF1MAF3-AGL31, PHYA, and RGA-RGA1 (Supplemental Figs. 29-62; Supplemental Tables 6 and 7). MP analyses with gaps recovered some of the species relationships within section Prunocerasus, the American Clade and Chickasaw Clade, as previously identified using isozymes and tree architecture gene sequences.

AGL20-SOC1, BFT, BRM, CO, FD, FD1, FPF1, HOS1, MAF1-MAF3-AGL31, MAF2B, MAF4, MAF5, MFT, PHYA, PHYB-PHYD, RGA-RGA1, RGL1-RLG2-RLG3, TFL1-ATC, and VRN2, did not identify any novel relationships within section Prunocerasus. MP and ML analysis of phased haplotype data for these genes produced more species clusters to those produced using diploid data. Prunus munsoniana and $P$. angustifolia were clustered together (in some occasions also with $P$. umbellata). These additional groupings were not consistent across genes (Supplemental Figs. 30-62). Additional results per gene region can be found in Supplemental Information.

MP and ML analyses of combined vernalization-related genes diploid data recovered section Prunocerasus as a monophyletic group. Prunus angustifolia and P. munsoniana were clustered together in all analyses (Chickasaw Clade). Similar relationships were identified for $P$. americana and $P$. mexicana, and $P$. maritima and $P$. umbellata. MP analyses (with gaps) yielded three major groups: 1) Chickasaw Clade ( $P$. angustifolia and $P$. munsoniana) and $P$. geniculata; 2) $P$. umbellata and $P$. maritima; and 3 ) American Clade ( $P$. mexicana, $P$. americana, and $P$. hortulana). ML analyses of combined data recovered similar species groups to those produced by MP analysis with gaps. Prunus pumila was identified as sister species to section Prunocerasus (Supplemental Fig. 67).

The nonstandarized multilocus (phased haplotype) combined tree architecture genes sequence data network (Supplemental Fig. 68) recovered similar species relationships to those produced by MP (with gaps) and ML analyses. Prunus maritima was clustered to $P$. umbellata (similar to isozymes and tree architecture genes combined data analyses). Additional sequence data information and models of sequence evolution are available in Supplemental Tables 5, 6, 7, 9, and 11.

MP and ML analyses of the combined diploid sequence data for the nuclear genes (Fig. 6) recovered section Prunocerasus as a monophyletic group (similar to combined analyses of isozymes, tree architecture, and vernalization-related genes; Supplemental Figs. 63, 65, and 67, respectively). Prunus angustifolia and $P$. munsoniana were clustered together in all analyses (Chickasaw Clade). Prunus americana was paired with $P$. mexicana, and $P$. maritima with $P$. umbellata. Similarly, the nonstandarized multilocus sequence data network for the combined nuclear genes recovered similar relationships to those produced by MP and ML (Fig. 7).

\section{Total evidence}

The use of cytoplasmic and nuclear markers using the total evidence approach (consensus phylogeny) would allow for the tracking of maternal and paternal lineages present among species. A total of 27,909 bp of alignable sequence (1945
PICS, polymorphism rate of $6.97 \%$ ) was obtained for cpDNA, ITS, and nuclear genes (Tables 3 and 4). Gene regions were summarized based on their polymorphism rate (percent variability within section Prunocerasus and the outgroup species. Sequence information represented both intron(s) and exon(s). As expected, intron(s) had the highest polymorphism rate across the core collection of 11 Prunus species (Fig. 3B; Supplemental Table 5).

Total evidence (combined diploid sequence data for cpDNA, ITS, and nuclear genes) MP (with gaps) analyses yielded two major groups: 1) American Clade (P. mexicana, $P$. americana, and $P$. hortulana) and $P$. geniculata and 2) Chickasaw Clade (P. angustifolia, P. munsoniana, and P. umbellata) and P. maritima. ML analyses of combined data recovered similar species groups to those recovered using MP analysis with gaps. Prunus pumila was identified as sister species to section Prunocerasus (Fig. 8).

The nonstandarized multilocus (phased haplotype) sequence data network for the combined tree architecture genes sequence data (Fig. 9) recovered similar species relationships to those produced by MP (with gaps) and ML analyses. Prunus maritima was clustered to $P$. umbellata (similar to isozymes, tree architecture, and vernalization-related genes combined data analyses). Additional sequence data information and models of sequence evolution are available in Supplemental Tables 5-11.

\section{Discussion}

The current discussion will be focused in the use of SSR, cpDNA, ITS, and nuclear genes for the phylogenetic analyses of a core collection of North American plum species to differentiate species relationships. Although, our results included more specific details of the analyses for each one of the gene regions, the discussion is focused on the applicability of these regions to determine species relationships.

Chloroplast DNA has been extensively used for phylogenetic studies in Prunus (Badenes and Parfitt, 1995; Bortiri et al., 2001, 2002, 2006; Kaneko et al., 1986; Katayama and Uematsu, 2005; Shaw and Small, 2004, 2005; Wen et al., 2008). However, evidence for hybridization among Prunus species may not be detected if the analysis is based solely on cpDNA information. Human disturbance, relocation of species, and other factors could increase contact between previously isolated species and facilitate their hybridization.

The existence of hybrids of native plum species with other Prunus species has been reported by Wight (1915) based on morphological traits. Wight (1915) reported that $P$. americana hybridized with $P$. angustifolia, Prunus besseyi (i.e., $P$. pumila), P. hortulana, P. munsoniana, and $P$. simonnii. Similarly, $P$. angustifolia hybridized freely with $P$. americana, Prunus triflora, P. besseyi, P. munsoniana, P. cerasifera, and $P$. orthosepala.

Shaw et al. $(2005,2007)$ identified chloroplast regions that provided the greatest number of characters for low-level molecular phylogenetic studies. From those, seven regions (out of eight) were sequenced for our core collection of 11 species. Our polymorphism rate estimates were different to those reported by Shaw et al. $(2005,2007)$ results. They used $P$. hortulana, $P$. nigra, and Prunus virginiana L. to determine the number of PICS. Prunus hortulana and P. nigra belong to subgenus Prunus section Prunocerasus and P. virginiana to subgenus Cerasus. 

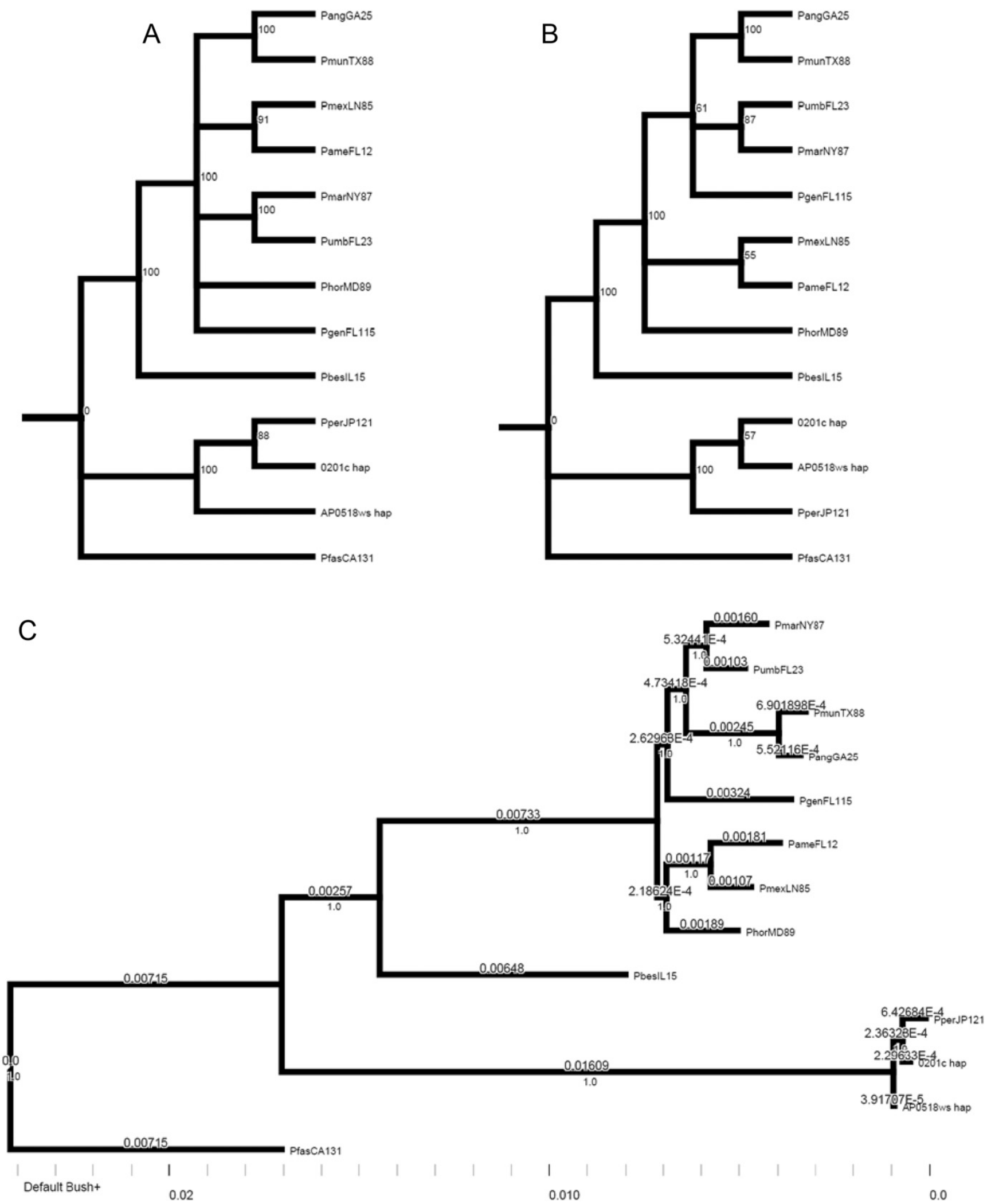

0.010

0.0

Fig. 6. Phylogenetic analyses of combined nuclear genes diploid sequence data (23,429 bp). Majority rule consensus trees using (A) maximum parsimony [2 trees, 1400 steps, consistency index $(\mathrm{CI})=0.913$, retention index $(\mathrm{RI})=0.920$, rescaled consistency index $(\mathrm{RC})=0.840]$ and $(\mathbf{B})$ maximum parsimony (including gaps) ( 1 tree, 2535 steps, $\mathrm{CI}=0.879, \mathrm{RI}=0.881, \mathrm{RC}=0.775)$ analyses. Bootstrap values greater than $50 \%$ are described above the branches. $(\mathbf{C})$ Maximum likelihood tree $(-\operatorname{lnL}=41,509.34)$. Branch lengths are described above the branches and posterior probability values below the branches. Trees are rooted with Prunus fasciculata. (PfasCA131). Other species in the cladogram are: P. americana (PameFL12), P. angustifolia (PangGA25), P. geniculata (PgenFL115), P. hortulana (PhorMD89), P. maritima (PmarNY87), P. mexicana (PmexLN85), P. munsoniana (PmunTX88), P. persica (PperJP121, 0201c_hap, AP0518ws_hap), P. pumila (PbesIL15), and P. umbellata (PumbFL23). 
A
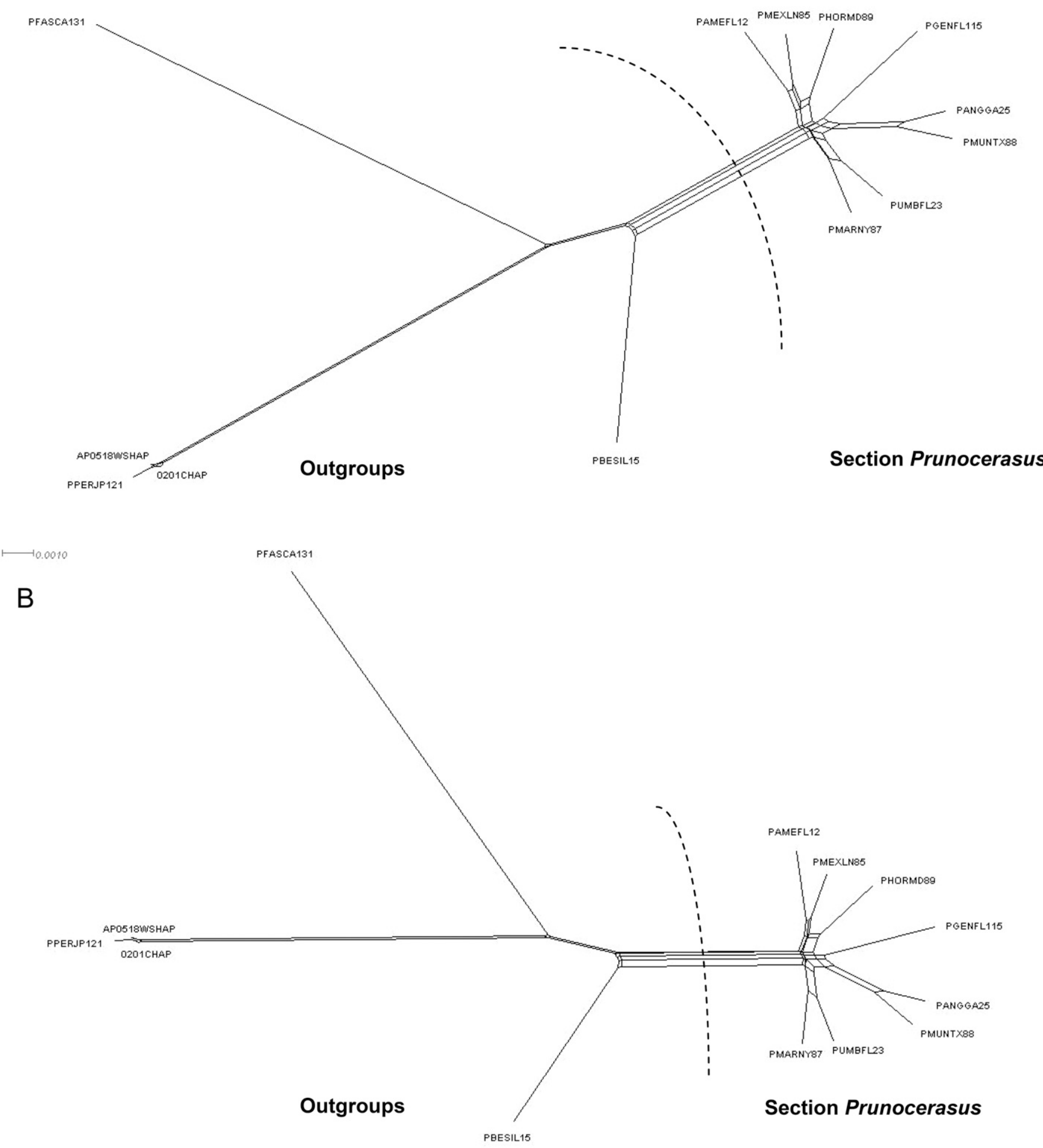

Fig. 7. A nonstandarized multilocus combined nuclear genes sequence data network using (A) gaps as missing characters and (B) gaps as fifth characters. Prunus species in the cladogram are: P. americana (PameFL12), P. angustifolia (PangGA25), P. fasciculata (PfasCA131), P. geniculata (PgenFL115), P. hortulana (PhorMD89), P. maritima (PmarNY87), P. mexicana (PmexLN85), P. munsoniana (PmunTX88), P. persica (PperJP121, 0201c_hap, AP0518ws_hap), P.pumila (PbesIL15), and P. umbellata (PumbFL23). 

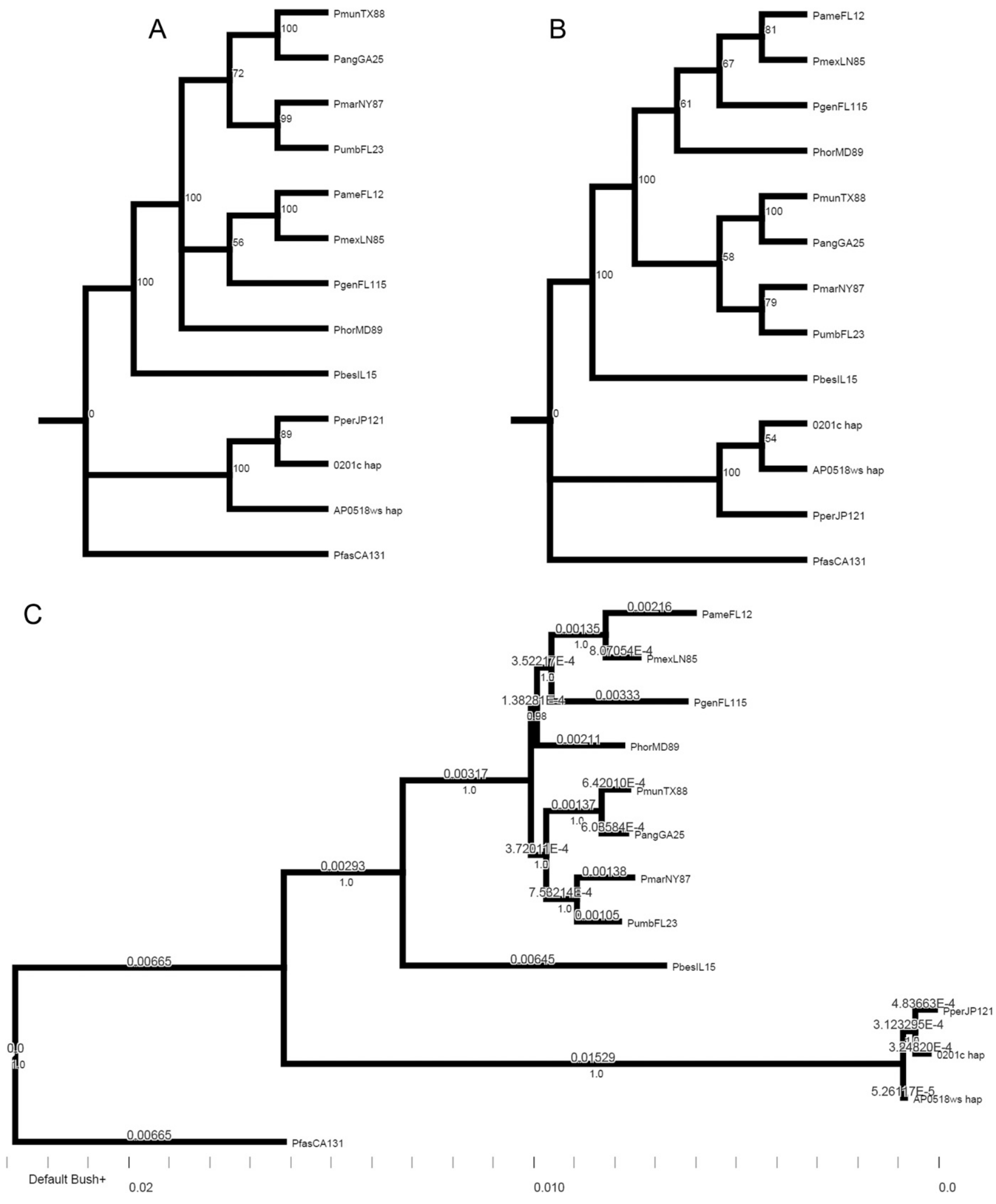

Fig. 8. Phylogenetic analyses of combined nuclear + chloroplast DNA + internal transcribed spacer diploid sequence data (27,623 bp). Majority rule consensus trees using $(\mathbf{A})$ maximum parsimony [1 tree, 1,594 steps, consistency index $(\mathrm{CI})=0.908$, retention index $(\mathrm{RI})=0.910$, rescaled consistency index $(\mathrm{RC})=0.827]$ and (B) maximum parsimony (including gaps) $(2$ trees, 2732 steps, $\mathrm{CI}=0.878, \mathrm{RI}=0.875, \mathrm{RC}=0.768)$ analyses. Bootstrap values greater than $50 \%$ are described above the branches. (C) Maximum likelihood tree $(-\operatorname{lnL}=48,496.34)$. Branch lengths described above the branches and posterior probability values below the branches. Trees are rooted with Prunus fasciculata (PfasCA131). Other species in the cladogram are: P. americana (PameFL12), P. angustifolia (PangGA25), $P$. geniculata (PgenFL115), P. hortulana (PhorMD89), P. maritima (PmarNY87), P. mexicana (PmexLN85), P. munsoniana (PmunTX88), P. persica (PperJP121, 0201c_hap, AP0518ws_hap), P. pumila (PbesIL15), and P. umbellata (PumbFL23). 
A
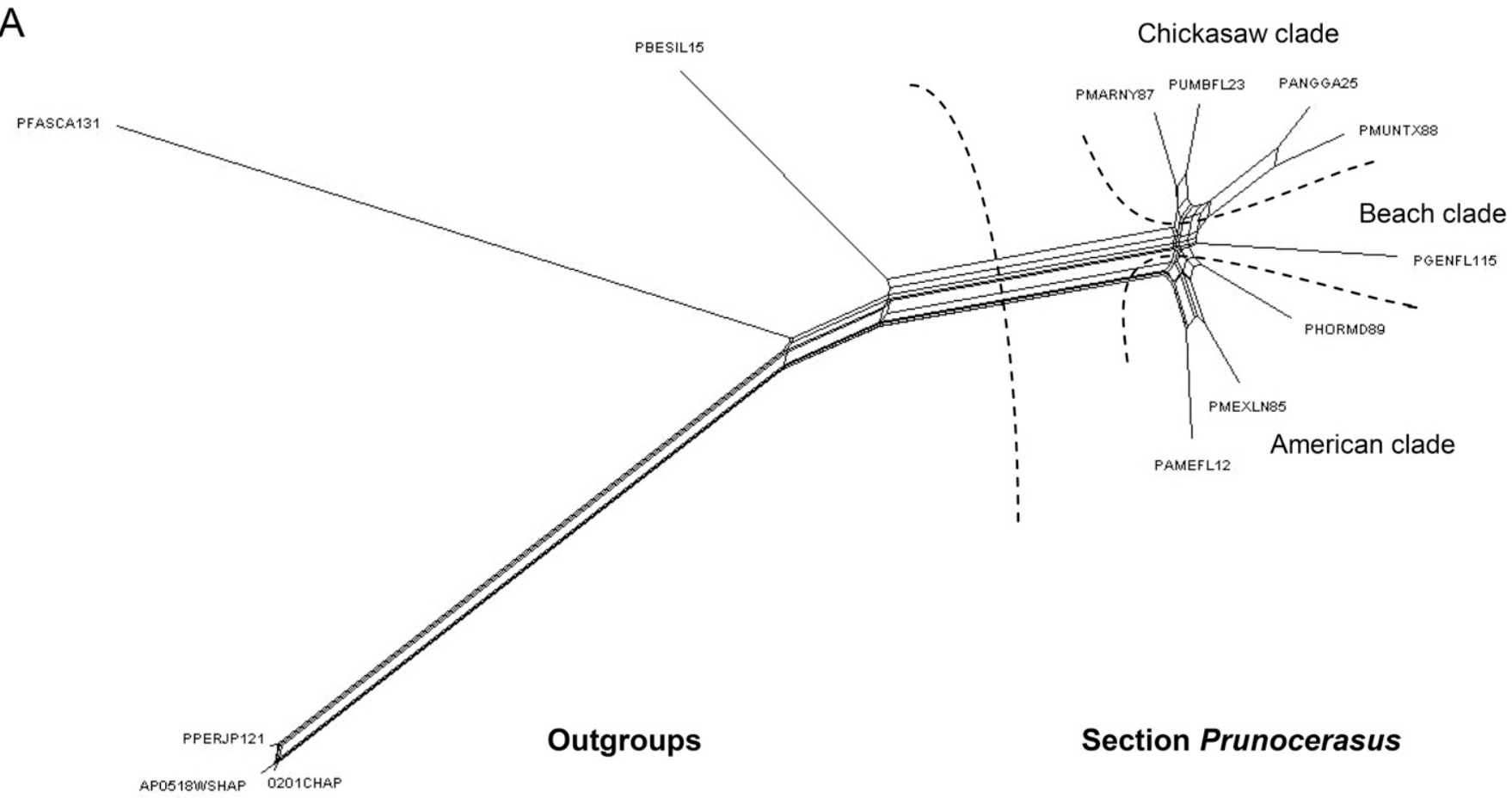

B
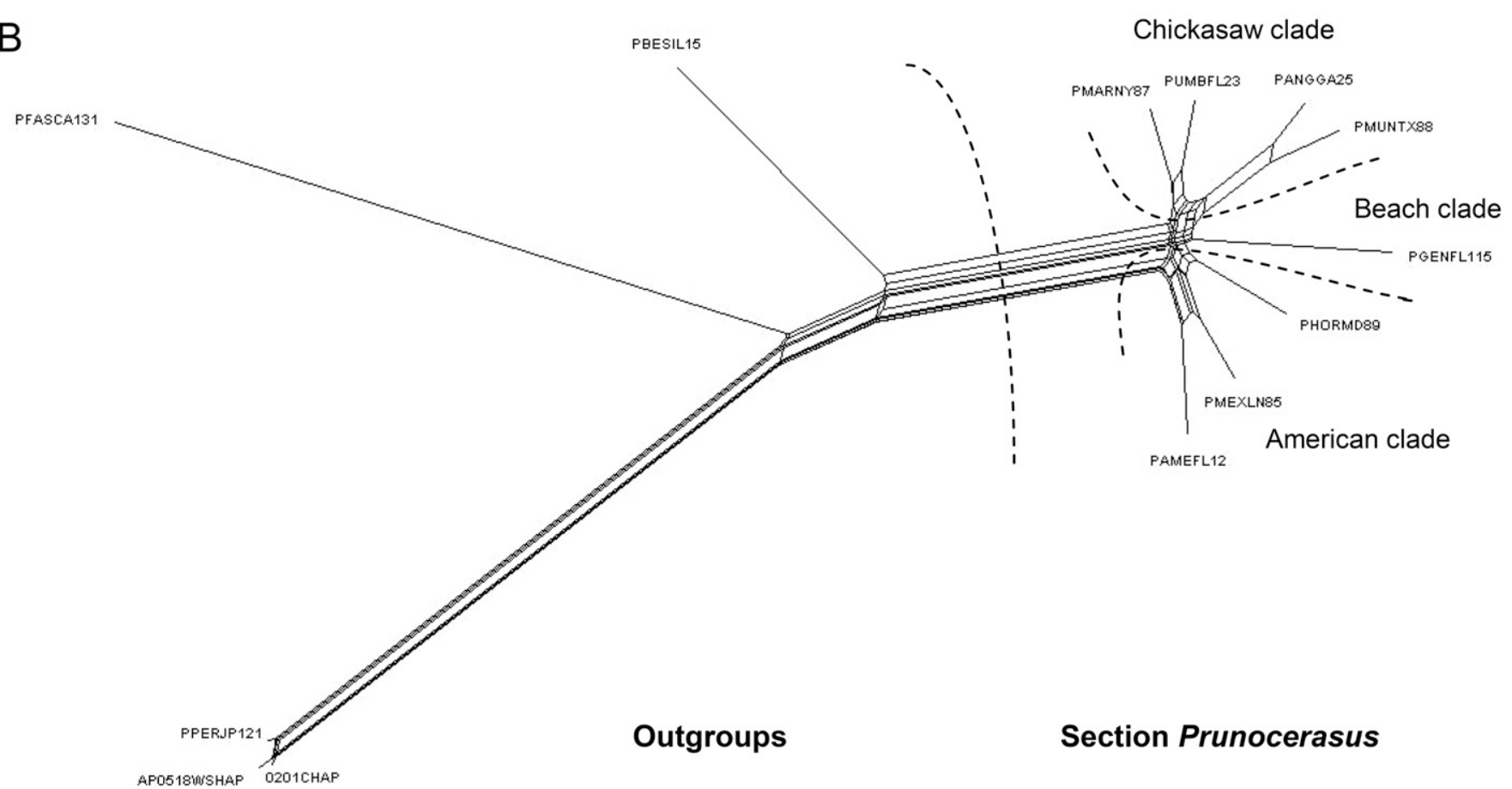

Fig. 9. A nonstandarized multilocus combined nuclear + chloroplast DNA + internal transcribed spacer sequence data network using (A) gaps as missing characters and (B) gaps as fifth characters. Prunus species in the cladogram are: P. americana (PameFL12), P. angustifolia (PangGA25), P. fasciculata (PfasCA131), P. geniculata (PgenFL115), P. hortulana (PhorMD89), P. maritima (PmarNY87), P. mexicana (PmexLN85), P. munsoniana (PmunTX88), P. persica (PperJP121, 0201c_hap, AP0518ws_hap), P. pumila (PbesIL15), and P. umbellata (PumbFL23). 
The regions with the highest PICS proved to be beneficial for the recovery of the phylogenetic relationships within section Prunocerasus (based on Shaw and Small, 2004).

Our analyses showed that the highest polymorphism rate (indels and substitutions) were mostly found at the outgroup level (Tables 3 and 4; Fig. 3). The limited number of species within section Prunocerasus and the broad comparison of levels across the genus Prunus was probably the reason for the higher polymorphism rates reported by Shaw et al. (2005, 2007). The highest frequency of informative sites in our study was observed in $3^{\prime}$ trn $V$ - $n d h C$ and trnH-psbA cpDNA regions (Fig. 3). Similar results were reported by Shaw et al. (2005, 2007).

It is important to recognize that although PICS are a good measure of how informative a region could be for a phylogenetic analysis, it did not represent the final phylogenetic relationships obtained from that region. Both methods were combined to select regions that could be used across species levels to identify phylogenetic patterns.

The presence of common alleles between the different species could be evidence for interspecific hybridization and gene flow between species. The use of cytoplasmic and nuclear markers would allow for the tracking of maternal and paternal lineages present in a more diverse phylogenetic study (Chavez, 2013).

Rohrer et al. (2004) analyzed the values of SSRs to resolve the relationships within Prunus at the species level. He concluded that the resolution of species relationships within section Prunocerasus required the use of regions more variable than ITS and less variable than SSRs. In our study, the outgroup species clustered together using 41 and six (highest PIC values) SSRs (Fig. 2). Cluster analyses using information from the 41 SSRs supported $P$. angustifolia and $P$ munsoniana as sister species to $P$. mexicana and $P$. geniculata. Similarly, $P$. americana, $P$. maritima, and $P$. umbellata clustered together. No previously reported relationships (such as the American Clade, Beach Clade, and Chickasaw Clade) were obtained.

Analysis of the species with the six SSR primers with the highest PIC values identified $P$. mexicana as sister species to all the outgroup species. The Beach Clade $(P$. geniculata and $P$. maritima) and Chickasaw Clade ( $P$. angustifolia and $P$. munsoniana) were recovered. Prunus americana and $P$. umbellata were monophyletic, and $P$. hortulana was found basal to all the section Prunocerasus Clade (Fig. 2).

The SSR data indicated a high level of heterezygosity as described by Rohrer et al. (2004). The use of SSRs distributed across the genome was expected to provide a sample of different levels of polymorphisms. However, it was observed that the use of six SSR markers (with the highest PIC values) yielded more consistent results than the analyses with 41 SSR markers, in comparison with known phylogenetic relationships in section Prunocerasus (Shaw and Small, 2004).

The observed level of heterozygosity for all genotypes was high compared with University of Florida's peach germplasm, 0.58 and 0.41, respectively (Chavez et al., 2014). Wild Prunus species typically express gametophythic self-incompatibility, making them highly heterozygous. Our results were concordant with these expectations.

ITS results were consistent with the remarks of Rohrer et al. (2004). He was not able to define the species relationships within section Prunocerasus using this region. In our study, ITS sequence data were an important baseline for comparison with other regions (nuclear and cpDNA). A polymorphism rate of
$10.82 \%$ was obtained for ITS (Table 4). This rate was intermediate between nuclear (high) and cpDNA (low) regions. The results obtained from the ITS phylogenetic results were consistent with our cpDNA results (Supplemental Fig. 7).

Three different groups of nuclear genes were used in this study: 1) isozymes, 2) tree architecture genes, and 3) vernalizationrelated genes. The isozyme genes were used due to their expected neutrality to selection and adaptation. The highest PICS values within section Prunocerasus and between outgroup were obtained for PGI, followed by s6pdh and PGDH (Table 4; Fig. 3). Section Prunocerasus was recovered as a monophyletic clade for all analyses (Supplemental Figs. 8-10; Supplemental Tables 6 and 7). These regions were more variable than cpDNA and less variable than ITS within section Prunocerasus (Fig. 3). Similar results were also obtained for tree architecture and vernalization-related genes (Supplemental Figs. 11-62; Supplemental Tables 6 and 7).

The tree architecture and vernalization-related genes were used due to their importance in plant development and expected selection and adaptation pressure. The highest PICS values within section Prunocerasus and between outgroup for tree architecture genes were obtained for MAX4, MAX1, CUC2, MAX3, and AXR1 (Table 4). Section Prunocerasus was recovered as a monophyletic clade with MP and ML analyses using diploid and haplotype datasets (Supplemental Figs. 11-28; Supplemental Tables 6 and 7). MP analyses with gaps did provide additional support (bootstrap) for some clades when using different genes. Similarly, the highest amount of PICS within section Prunocerasus and between outgroup for vernalization-related genes were observed in MAF4, FLC-FLF, LFY, MAF2A, AGL20-SOC1, ELF6, and AGL24 (Table 4). Section Prunocerasus was monophyletic in MP and ML analyses using diploid and haplotype datasets for all gene regions except FPF1, MAF1-MAF3-AGL31, PHYA, and RGA-RGA1 (Supplemental Figs. 29-62; Supplemental Tables 6 and 7). MP analyses with gaps recovered additional species grouping within section Prunocerasus.

MP and ML phased haplotype and the nonstandarized multilocus analyses yielded similar results as using diploid sequence data. This consistency across analyses and different gene regions supported the presence of a phylogenetic signal. It was observed that regions with the highest polymorphism rates within section Prunocerasus allowed identification of additional species relationships (clades), as compared with known clades in section Prunocerasus (Shaw and Small, 2004). These regions constituted good candidates for future phylogenetic relationships in Prunus.

MP and ML analyses of isozymes, tree architecture, and vernalization-related genes found two major groups, similar to those described by Waugh's (1901) classification of section Prunocerasus. The American Clade and Chickasaw Clade were identified in our analyses. Our results support section Prunocerasus as a monophyletic group using individual and combined analyses as previously reported by Bortiri et al. (2001) and Shaw and Small (2004). However, there was no evidence for $P$. maritima and $P$. geniculata being sister species (Beach Clade) as reported by Shaw and Small (2004). In the majority of the analyses, $P$. maritima was supported as a member of the Chickasaw Clade (in specific sister species to $P$. umbellata). Similarly, $P$. geniculata was identified as a member species of the American Clade (Supplemental Figs. 63-68; Figs. 6 and 7). 
Our analyses provided similar results to those reported by Mowrey and Werner (1990). They identified a clade formed by $P$. maritima and P. umbellata within section Prunocerasus based on isozyme data. In another study, Rohrer et al. (2008) used LEAFY and s6pdh sequences to examine the North American plums phylogeny. They reported that $P$. geniculata was clustered with $P$. texana. In addition, $P$. umbellata, $P$. maritima, $P$. alleghaniensis, and $P$. gracilis formed a paraphyletic group using LEAFY.

The use of different sequence regions, in a core collection of section Prunocerasus and outgroup species, constituted an important study to identify genome regions with the highest level (number) of PICS and the most valuable phylogenetic signal based on previously known clades within section Prunocerasus (Shaw and Small, 2004). Out of the 52 nuclear genes ( 3 isozyme, 16 tree architecture, and 33 vernalizationrelated genes), 7 cpDNA regions, and ITS sequences, trnH$p s b A$, PGI, MAX4, AXR1, LFY, PHYE, and VRN1, are recommended and chosen to be used for a phylogenetic analysis with a larger number of taxa. Regions with high polymorphism rates and strong phylogenetic signal as compared with known phylogenetic relationships within section Prunocerasus were trnH-psbA, PGI, MAX4, AXR1, and LFY. PHYE and VRN1 were regions with low polymorphism rates. However, they presented a strong phylogenetic signal (polymorphism within these regions were highly informative).

\section{Conclusions}

The identification and use of additional genomic regions that provided the greatest number of characters, greatest variability, and improved the phylogenetic signal at the low level in Prunus section Prunocerasus relationships was achieved. The PICS content was highly variable across regions, with nuclear genes being the highest, followed by ITS, and cpDNA regions. The species relationships within section Prunocerasus and outgroup species were recovered for the majority of regions in comparison with known clades as reported by Shaw and Small (2004). Our study yielded a set of candidate regions that could be used for future phylogenetic relationships in Prunus.

It is important to identify PICS as a good measure of how informative a region will be for phylogenetic analysis, but always complemented with the phylogenetic analysis by itself. A preliminary study should always include several species that fall in different levels of the genus of study. Both methods should be combined to select regions that will provide the most value.

These sequences could be used to create hybridization probes for microarray chips to study the presence or absence of certain polymorphic sites associated with vernalization response, and plant architecture and branching genes.

\section{Literature Cited}

Akaike, H. 1974. A new look at the statistical model identification criteria. IEEE Trans. Automat. Contr. 19:716-723.

Badenes, M.L. and D.E. Parfitt. 1995. Phylogenetic relationships of cultivated Prunus species from an analysis of chloroplast DNA variation. Theor. Appl. Genet. 90:1035-1041.

Benson, D.A., M. Cavanaugh, K. Clark, I. Karsch-Mizrachi, D.J. Lipman, J. Ostell, and E.W. Sayers. 2013. GenBank. Nucl. Acids Res. 41:D36-D42.

Bortiri, E., S.-H. Oh, F.-Y. Gao, and D. Potter. 2002. The phylogenetic utility of nucleotide sequences of sorbitol 6-phosphate dehydrogenase in Prunus (Rosaceae). Amer. J. Bot. 89:1697-1708.
Bortiri, E., S.-H. Oh, J. Jiang, S. Baggett, A. Granger, C. Weeks, M. Buckingham, D. Potter, and D.E. Parfitt. 2001. Phylogeny and systematic of Prunus (Rosaceae) as determined by sequence analysis of ITS and the chloroplast trnL-trnF spacer DNA. Syst. Bot. 26:797807.

Bortiri, E., B. Vanden Heuvel, and D. Potter. 2006. Phylogenetic analysis of morphology in Prunus reveals extensive homoplasy. Plant Syst. Evol. 259:53-71.

Carrillo-Mendoza, O. 2012. Genetics of tree architecture in peach (Prunus persica (L.) Batsch). PhD Diss., Univ. Florida, Gainesville, FL.

Chavez, D.J. 2013. The North American plums (Prunus spp.) and their use as germplasm resources: From populations to phylogenetic studies - A breeder's perspective. PhD Diss., Univ. Florida, Gainesville, FL.

Chavez, D.J., T.G. Beckman, D.J. Werner, and J.X. Chaparro. 2014. Genetic diversity in peach [Prunus persica (L.) Batsch] at the University of Florida: Past, present and future. Tree Genet. Genomes 10:1399-1417.

Chavez, D.J. and J.X. Chaparro. 2011. Identification of markers linked to seedlessness in Citrus kinokuni hort. ex Tanaka and its progeny using bulked segregant analysis. HortScience 46:693-697.

Chiang, T.-Y., B.A. Schaal, and C.-I. Peng. 1998. Universal primers for amplification and sequencing a noncoding spacer between atpB and rbcL genes of chloroplast DNA. Botanical Bul. Acad. Sinica. 39:245-250.

Clement, M., D. Posada, and K.A. Crandall. 2000. TCS: A computer program to estimate gene genealogies. Mol. Ecol. 9:1657-1659.

Dirlewanger, E., E. Graziano, T. Joobeur, F. Garriga-Caldere, P. Cosson, W. Howad, and P. Arús. 2004. Comparative mapping and marker-assisted selection in Rosaceae fruit crops. Proc. Natl. Acad. Sci. USA 101:9891-9896.

Edgar, R.C. 2004. MUSCLE: Multiple sequence alignment with high accuracy and high throughput. Nucl. Acids Res. 32:1792-1797.

Felsenstein, J. 1985. Confidence limits on phylogenies: An approach using the bootstrap. Evolution 39:783-791.

Goodstein, D.M., S. Shu, R. Howson, R. Neupane, R.D. Hayes, J. Fazo, T. Mitros, W. Dirks, U. Hellsten, N. Putnam, and D.S. Rokhsar. 2012. Phytozome: A comparative platform for green plant genomics. Nucl. Acids Res. 40:D1178-D1186.

Hedrick, U.P. 1911. The plums of New York. Rpt. New York State Agr. Expt. Sta. Albany, J.B. Lyon, Geneva, NY.

Huson, D.H. and D. Bryant. 2006. Application of phylogenetic networks in evolutionary studies. Mol. Biol. Evol. 23:254-267.

Joly, S. and A. Bruneau. 2006. Incorporating allelic variation for reconstructing the evolutionary history of organisms from multiple genes: An example from Rosa in North America. Syst. Biol. 55:623636.

Jung, S., M. Staton, T. Lee, A. Blenda, R. Svancara, A. Abbott, and D. Main. 2008. GDR (genome database for Rosaceae): Integrated webdatabase for Rosaceae genomics and genetic data. Nucl. Acids Res. 36:1034-1040.

Kaneko, T., T. Terachi, and K. Tsunewaki. 1986. Studies on the origin of crops species by restriction endonuclease analysis of organellar DNA. II. Restriction analysis of ctDNA of 11 Prunus species. Jpn. J. Genet. 61:157-168.

Katayama, H. and C. Uematsu. 2005. Structural analysis of chloroplast DNA in Prunus (Rosaceae): Evolution, genetic diversity and unequal mutations. Theor. Appl. Genet. 111:1430-1439.

Lee, S. and J. Wen. 2001. A phylogenetic analysis of Prunus and the Amygdaloideae (Rosaceae) using ITS sequences of nuclear ribosomal DNA. Amer. J. Bot. 88:150-160.

Librado, P. and J. Rozas. 2009. DnaSP v5: A software for comprehensive analysis of DNA polymorphism. Bioinformatics 25:14511452.

Liu, K. and S.V. Muse. 2005. PowerMarker: An integrated analysis environment for genetic marker analysis. Bioinformatics 21:21282129. 
Maddison, W.P. and D.R. Maddison. 2011. Mesquite: A modular system for evolutionary analysis. Version 2.75. 20 Dec. 2012. $<$ http://mesquiteproject.org $>$.

Mason, S.C. 1913. The pubescent-fruited species of Prunus of the southwestern states. J. Agr. Res. 1:147-179.

Miller, M.A., W. Pfeiffer, and T. Schwartz. 2010. Creating the CIPRES science gateway for inference of large phylogenetic trees. Proc. Gateway Computing Environ. Workshop. 14 Nov. 2010. New Orleans, LA. p. 1-7.

Morris, A.B., S.M. Ickert-Bond, D.B. Brunson, D.E. Soltis, and P.S. Soltis. 2008. Phylogeographic structure and temporal complexity in American sweetgum (Liquidambar styraciflua). Mol. Ecol. 17:38893900 .

Mowrey, B.D. and D.J. Werner. 1990. Phylogenetic relationships among species of Prunus as inferred by isozyme markers. Theor. Appl. Genet. 80:129-133.

Nei, M. and N. Takezaki. 1983. Estimation of genetic distances and phylogenetic trees from DNA analysis. Proc. 5 World Congr. Genet. Appl. Livestock Prod. 21:405-412.

Oatley, G., G. Voelker, T.M. Crowe, and R.C.K. Bowie. 2012. A multi-locus phylogeny reveals a complex pattern of diversification related to climate and habitat heterogeneity in southern African white-eyes. Mol. Phylogenet. Evol. 64:633-644.

Peakall, R. and P.E. Smouse. 2006. GENALEX 6: Genetic analysis in Excel. Population genetic software for teaching and research. Mol. Ecol. Notes 6:288-295.

Peakall, R. and P.E. Smouse. 2012. GenAlEx 6.5: Genetic analysis in Excel. Population genetic software for teaching and research-An update. Bioinformatics 28:2537-2539.

Posada, D. 2008. JModeltest: Phylogenetic model averaging. Mol. Biol. Evol. 25:1253-1256.

Rehder, A. 1940. Manual of cultivated trees and shrubs hardy in North America. 2nd ed. Macmillan, New York, NY.

Rohrer, J.R., R. Ahmad, S.M. Southwick, and D. Potter. 2004. Microsatellite analysis of relationships among North American plums (Prunus section Prunocerasus, Rosaceae). Plant Syst. Evol. 244:69-75.

Rohrer, J.R., M.A. O'Brien, and J.A. Anderson. 2008. Phylogenetic analysis of North American plums (Prunus section Prunocerasus: Rosaceae) based on nuclear LEAFY and S6PDH sequences. J. Bot. Res. Inst. Tex. 2:401-414.

Saitou, N. and M. Nei. 1987. The neighbor-joining method: A new method for reconstructing phylogenetic trees. Mol. Biol. Evol. 4: 406-425.

Sang, T., D.J. Crawford, and T.F. Stuessy. 1997. Chloroplast DNA phylogeny, reticulate evolution, and biogeography of Paeonia (Paeoniaceae). Amer. J. Bot. 84:1120-1136.

Schwarz, G. 1978. Estimating the dimension of a model. Ann. Stat. 6:461-464.

Shaw, J., E.B. Lickey, J.T. Beck, S.B. Farmer, W. Liu, J. Miller, K.C. Siripun, C.T. Winder, E.D. Schilling, and R.L. Small. 2005. The tortoise and the hare II: Relative utility of 21 noncoding chloroplast DNA sequences for phylogenetic analysis. Amer. J. Bot. 92:142-166.
Shaw, J., E.B. Lickey, E.E. Schilling, and R.L. Small. 2007. Comparison of whole chloroplast genome sequences to choose noncoding regions for phylogenetic studies in angiosperms: The tortoise and the hare III. Amer. J. Bot. 94:275-288.

Shaw, J. and R.L. Small. 2004. Addressing the "hardest puzzle in the American pomology:" Phylogeny of Prunus section Prunocerasus (Rosaceae) based on seven noncoding chloroplast DNA regions. Amer. J. Bot. 91:985-996.

Shaw, J. and R.L. Small. 2005. Chloroplast DNA phylogeny and phylogeography of the North American Plums (Prunus subgenus Prunus section Prunocerasus, Rosaceae). Amer. J. Bot. 92:20112030.

Small, R.L., J.A. Ryburn, R.C. Cronn, T. Seelanan, and J.F. Wendel. 1998. The tortoise and the hare: Choosing between noncoding plastome and nuclear Adh sequences for phylogeny reconstruction in a recently diverged plant group. Amer. J. Bot. 85:1301-1315.

Soltis, D.E. and P.S. Soltis. 1998. Choosing and approach and an appropriate gene for phylogenetic analysis, p. 1-42. In: D.E. Soltis, P.S. Soltis, and J.J. Doyle (eds.). Molecular systematics of plants II: DNA sequencing. Kluwer, Norwell, MA.

Stamatakis, A. 2006. RAxML-VI-HPC: Maximum likelihood-based phylogenetic analyses with thousands of taxa and mixed models. Bioinformatics 22:2688-2690.

Stamatakis, A., P. Hoover, and J. Rougemont. 2008. A fast bootstrapping algorithm for the RAxML web-servers. Syst. Biol. 57:758-771.

Swofford, D.L. 2002. PAUP*: Phylogenetic analysis using parsimony (* and other methods) version 4.0b10. Sinauer Assoc., Sunderland, MA.

Taberlet, P., L. Gielly, G. Pautou, and J. Bouvet. 1991. Universal primers for amplification of three non-coding regions of chloroplast DNA. Plant Mol. Biol. 17:1105-1109.

Tate, J.A. and B.B. Simpson. 2003. Paraphyly of Tarasa (Malvaceae) and diverse origins of the polyploid species. Systematic Bot. 28:723737.

Untergasser, A., I. Cutcutache, T. Koressaar, J. Ye, B.C. Faircloth, M. Remm, and S.G. Rozen. 2012. Primer3-new capabilities and interfaces. Nucl. Acids Res. 40:e115.

Waugh, F.A. 1901. Plums and plum culture. Orange Judd, New York, NY.

Wen, J., S.T. Berggren, C.-H. Lee, S. Ickert-Bond, T.-S. Yi, K.-O. Yoo, L. Xie, J. Shaw, and D. Potter. 2008. Phylogenetic inferences in Prunus (Rosaceae) using chloroplast $n d h F$ and nuclear ribosomal ITS sequences. J. Syst. Evol. 46:322-332.

Wen, J. and E.A. Zimmer. 1996. Phylogeny and biogeography of Panax L. (the ginseng genus, Araliaceae): Inferences from ITS sequences of nuclear ribosomal DNA. Mol. Phylogenet. Evol. 6:166177 .

Wight, W.F. 1915. Native American species of Prunus. U.S. Dept. Agr. Bul. 179.

Zwickl, D.J. 2006. Genetic algorithm approaches for the phylogenetic analysis of large biological sequence datasets under the maximum likelihood criterion. PhD Diss., Univ. Texas, Austin, TX. 


\section{Supplemental Information}

Tree ArChItecture. AXR1 recovered the American Clade relationships in diploid sequence analyses using maximum parsimony (MP) and maximum likelihood (ML). In haploid sequence analyses, two major groups were identified: 1) Prunus maritima, Prunus umbellata, and Prunus angustifolia, which shared the same haplotype sequence and 2) Prunus angustifolia (additional haplotype), Prunus geniculata, Prunus munsoniana, Prunus mexicana, Prunus americana, and Prunus hortulana. Both groups represented the Chickasaw Clade and the American Clade. P. geniculata and P. angustifolia haplotypes contained haplotypes in each clade (Supplemental Fig. 11). AXR1 recovered the American Clade and the Chickasaw Clade within section Prunocerasus and constituted a good candidate for further studies in genus Prunus.

BRC1, BRC2, and CUC1 (CUC1A and CUC1B) sequence data provided limited information for elucidating species relationships within section Prunocerasus. MP analyses recovered section Prunocerasus as a monophyletic group [except for CUC1 (Supplemental Figs. 12-15)]. The polymorphism rate of these genes was similar to some of the cpDNA regions. These regions could be candidates for analyses at the subgenus/ genus level in Prunus.

Analysis of CUC2 recovered section Prunocerasus as monophyletic group in MP and ML analyses using diploid and phased haplotype sequence data. Prunus americana, $P$. maritima, and $P$. mexicana clustered together in both analyses (Supplemental Fig. 16). Similarly, CUC3 phylogenetic analyses differentiated section Prunocerasus from the outgroup species for MP and ML analyses. Only the ML analyses of the diploid sequence data yielded monophyletic clades that supported the Beach Clade (with $P$. umbellata joined to this clade), the American Clade, and the Chickasaw Clade within section Prunocerasus. Haplotype sequence analyses recovered species haplotypes in different clades (Supplemental Fig. 17).

LAS analyses yielded a monophyletic clade for section Prunocerasus. The diploid MP analyses did not identify additional relationships. Diploid ML and phased haplotype MP-ML analyses presented $P$. americana and $P$. mexicana clustered together. Similarly, $P$. angustifolia, $P$. umbellata, and $P$. munsoniana (from the Chickasaw Clade) were clustered together (Supplemental Fig. 18).

Analysis of diploid and haplotype sequence for MAX1 [MAX1A and MAX1B (only for MP)] also identified section Prunocerasus as a monophyletic group, but it did not provide any additional resolution to species relationships within section Prunocerasus (Supplemental Figs. 19 and 20). Similarly, MAX2, MAX3, PIN, and RAX1 did not recover any species relationships within section Prunocerasus (Supplemental Figs. 21, 22, 24, and 25).

MAX4 identified section Prunocerasus as a monophyletic clade. Two major groups were recovered within section Prunocerasus in all analyses: 1) American Clade species and P. geniculata and 2) Chickasaw Clade species, Prunus pumila and $P$. maritima (Supplemental Fig. 23). This was the region with the highest percent variability. MP and ML recovered several species clusters within section Prunocerasus. MAX4 is a good candidate for a whole phylogenetic analysis to identify additional species clades in section Prunocerasus.

RAX2-RAX3 recovered Chickasaw Clade relationships in diploid sequence analyses using MP (with gaps) and ML.
Prunus maritima, P. umbellata, and P. angustifolia shared the same haplotype sequence and were clustered together (Supplemental Fig. 26). The phylogenetic relationships identified with RAX2-RAX3 were somewhat similar to those recovered with AXR1. No additional species groupings were obtained.

REV differentiated section Prunocerasus from the outgroup species in MP and ML analyses. The American Clade species, $P$. americana and $P$. mexicana, were clustered together in MP analyses. Additional species relationships within section Prunocerasus were recovered when using phased haplotype sequences (Supplemental Fig. 27).

Vernalization. MP clustered the Chickasaw Clade species and $P$. geniculata together using the AGL24 diploid and phased haplotype data. ML analyses did not yield similar results. Prunus maritima was located near the base of section Prunocerasus. Haplotype MP phylogenetic analysis recovered the American Clade and the Chickasaw Clade (Supplemental Fig. 29). The use of gaps in this analysis did not provide additional information. This region had some difficulties during sequencing due to high polymorphisms. Additional internal primers could be created to improve sequence quality and coverage.

CRY1 identified section Prunocerasus as a monophyletic group in MP analyses with diploid and phased haplotype sequence data. Prunus munsoniana and P. angustifolia were clustered together (Chickasaw Clade). This clade was sister to the other species within section Prunocerasus. Prunus maritima and $P$. umbellata were identified in a cluster. No additional species relationships were observed (Supplemental Fig. 34).

CRY2, ELF6, and FG diploid data analyses generated somewhat similar results than CRY1 (Supplemental Figs. 3537). Prunus munsoniana and $P$. angustifolia were clustered together (Chickasaw Clade). However, different species relationships were identified when using haplotype sequence data for each region. ELF6 MP analyses (with gaps) for diploid and haplotype data yielded more species clusters than their counterpart. FG phased haplotype MP and ML analyses recovered the Americana Clade and Chickasaw Clade relationships. No additional clusters were identified across these genes.

FLC-FLF recovered section Prunocerasus as a monophyletic clade. Additional species relationships within this section were not consistent, with MP (with and without gaps) and ML analyses producing different results (Supplemental Fig. 40). This region did not improve species phylogenetic relationships within section Prunocerasus, although this region had high polymorphism rate in comparison with other gene regions. Furthermore, $P$. americana, $P$. fasciculata, and $P$. pumila were not able to be sequenced with the primers designed (multiple fragments).

FRIGIDA phylogenetic analyses were not representative of the core collection species relationships as the $P$. umbellata, $P$. angustifolia, $P$. mexicana, $P$. hortulana, and $P$. munsoniana amplicons were not able to be sequenced. FRIGIDA MP and ML analyses of diploid and phased haplotype sequence data did recover section Prunocerasus as a monophyletic clade (Supplemental Fig. 42).

FT-TSF analyses supported section Prunocerasus as a monophyletic clade. MP analyses clustered $P$. angustifolia, $P$. munsoniana, and $P$. geniculata in one clade. Similarly, $P$. maritima and $P$. umbellata were identified as sister species in other clade. MP analyses using gaps (as additional characters) 
improved support for some clades. The American Clade was recovered. Analyses with phased haplotype sequence data yielded similar results. No major clades representing the American Clade and Chickasaw Clades within section Prunocerasus were found (Supplemental Fig. 43).

GI-FB analyses supported section Prunocerasus monophyly. Two major groups were obtained in MP (with gaps) and ML analyses using diploid and haplotype sequence data: 1) American Clade (P. americana, P. mexicana, and P. hortulana) and 2) Chickasaw Clade and Beach Clade species. Haplotype analyses did not show any additional groupings (Supplemental Fig. 44).

LFY identified section Prunocerasus as a monophyletic clade. Two major groups were recovered within section Prunocerasus for all analyses: 1) American Clade species and $P$. geniculata and 2) Chickasaw Clade species and $P$. maritima (Supplemental Fig. 46). This region had a high polymorphism rate and recovered several species relationships within section Prunocerasus as described above. LFY is a good candidate for a whole phylogenetic analysis within section Prunocerasus. Similar results were observed for MAF2A (Supplemental Fig. 48). MP analyses including gaps increased support for each clade. Diploid and phased haplotype results were similar.

PHYE analyses produced similar results that LFY and MAF2A phylogenetic results. One group represented Chickasaw Clade and Beach Clade species. The other group represented American Clade species. No additional support was obtained by using gaps in MP. Haplotype sequence data produced similar results to those produced by diploid data (Supplemental Fig. 55).

SPY-MP analyses with diploid and phased haplotype sequence data recovered $P$. americana and $P$. mexicana clustered together (American Clade). Similarly, $P$. angustifolia and $P$. munsoniana were found as sister species (Chickasaw Clade). ML analyses did not support any species relationships (Supplemental Fig. 58). Similarly, P. angustifolia and $P$. munsoniana were supported as sister species using TFL2 diploid and phased haplotype sequence data (Supplemental Fig. 60).

VRN1 supported section Prunocerasus monophyly. Two major groups were recovered within section Prunocerasus for all analyses: 1) American Clade species, P. umbellata and P. maritima and 2) Chickasaw Clade species. Prunus maritima was observed in a polytomy with the Chickasaw Clade (Supplemental Fig. 65). Results from diploid and haplotype sequence data were similar. No additional species clusters were identified using haplotype sequence data. VRN1 had a small polymorphism rate of $1.35 \%$ within section Prunocerasus. However, VRN1 is a good candidate for a whole phylogenetic analysis within section Prunocerasus, because its few amount of potentially informative characters represented a good phylogenetic signal. 

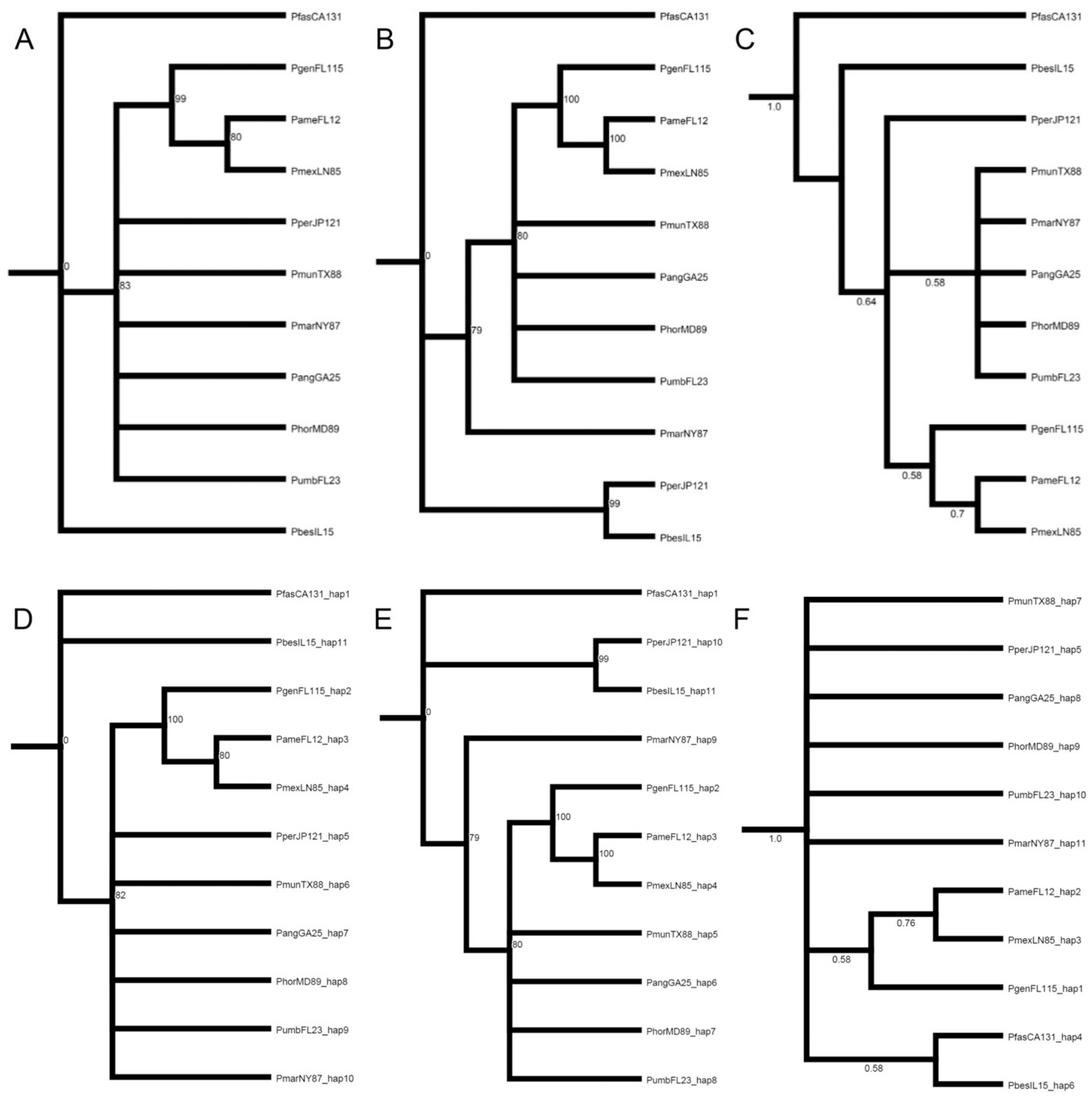

Supplemental Fig. 1. Majority rule consensus trees for $3^{\prime} \operatorname{trn} V$-ndhC chloroplast DNA region diploid sequence data using (A) maximum parsimony, (B) maximum parsimony (including gaps), and (C) maximum likelihood; and sequence data (with haplotype number prefix) using (D) maximum parsimony, (E) maximum parsimony (including gaps), and (F) maximum likelihood. Bootstrap values greater than $50 \%$ are described above the branches for maximum parsimony tree. Posterior probability values are described above the branches for maximum likehood tree. Trees are rooted with Prunus fasciculata (PfasCA131). Other species in the cladogram are Prunus americana (PameFL12), Prunus angustifolia (PangGA25), Prunus geniculata (PgenFL115), Prunus hortulana (PhorMD89), Prunus maritima (PmarNY87), Prunus mexicana (PmexLN85), Prunus munsoniana (PmunTX88), Prunus persica (PperJP121), Prunus pumila (PbesIL15), and Prunus umbellata (PumbFL23). 

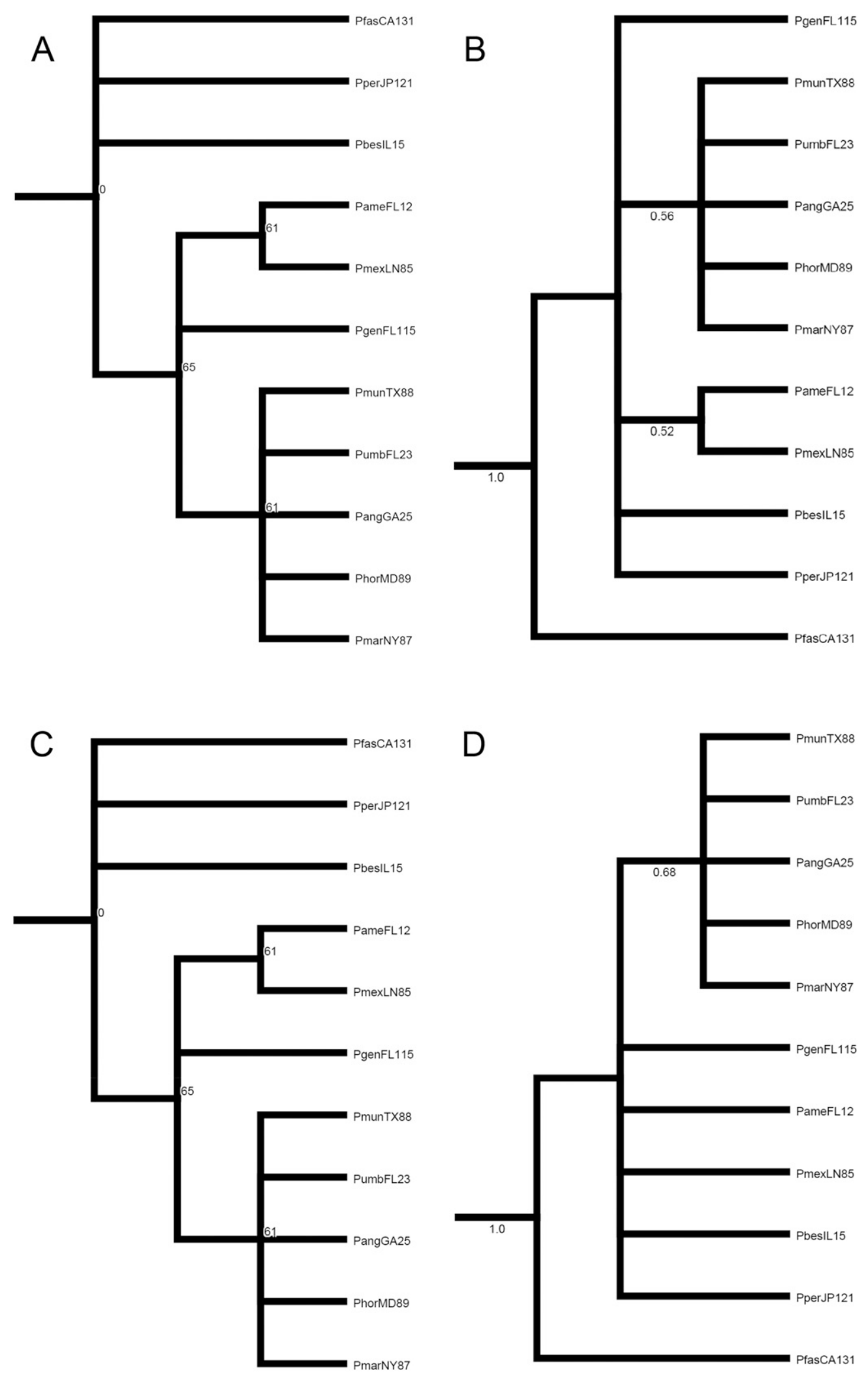

Supplemental Fig. 2. Majority rule consensus trees for $\operatorname{trn} L-\operatorname{trn} F$ and $\operatorname{trn} L$ intron chloroplast DNA regions diploid sequence data using (A) maximum parsimony and (B) maximum likelihood; and sequence data (haplotypes) using (C) maximum parsimony and (D) maximum likelihood. Bootstrap values greater than $50 \%$ are described above the branches for maximum parsimony tree. Posterior probability values are described above the branches for maximum likehood tree. Trees are rooted with Prunus fasciculata (PfasCA131). Other species in the cladogram are Prunus americana (PameFL12), Prunus angustifolia (PangGA25), Prunus geniculata (PgenFL115), Prunus hortulana (PhorMD89), Prunus maritima (PmarNY87), Prunus mexicana (PmexLN85), Prunus munsoniana (PmunTX88), Prunus persica (PperJP121), Prunus pumila (PbesIL15), and Prunus umbellata (PumbFL23). 

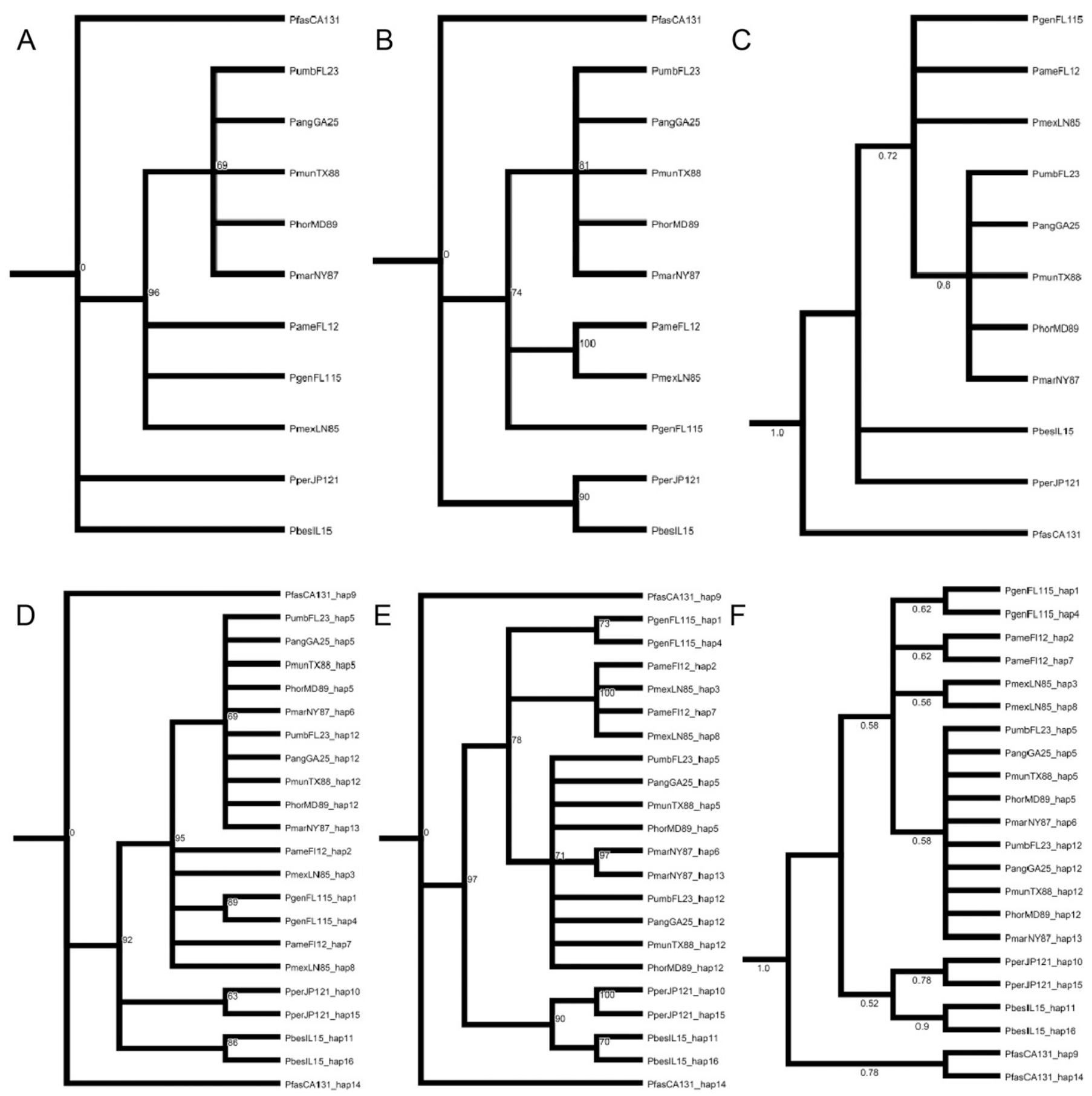

Supplemental Fig. 3. Majority rule consensus trees for trnQ-5'rps 16 chloroplast DNA region diploid sequence data using (A) maximum parsimony, (B) maximum parsimony (including gaps), and (C) maximum likelihood; and sequence data (with haplotype number prefix) using (D) maximum parsimony, (E) maximum parsimony (including gaps), and (F) maximum likelihood. Bootstrap values greater than $50 \%$ are described above the branches for maximum parsimony tree. Posterior probability values are described above the branches for maximum likehood tree. Trees are rooted with Prunus fasciculata (PfasCA131). Other species in the cladogram are Prunus americana (PameFL12), Prunus angustifolia (PangGA25), Prunus geniculata (PgenFL115), Prunus hortulana (PhorMD89), Prunus maritima (PmarNY87), Prunus mexicana (PmexLN85), Prunus munsoniana (PmunTX88), Prunus persica (PperJP121), Prunus pumila (PbesIL15), and Prunus umbellata (PumbFL23). 

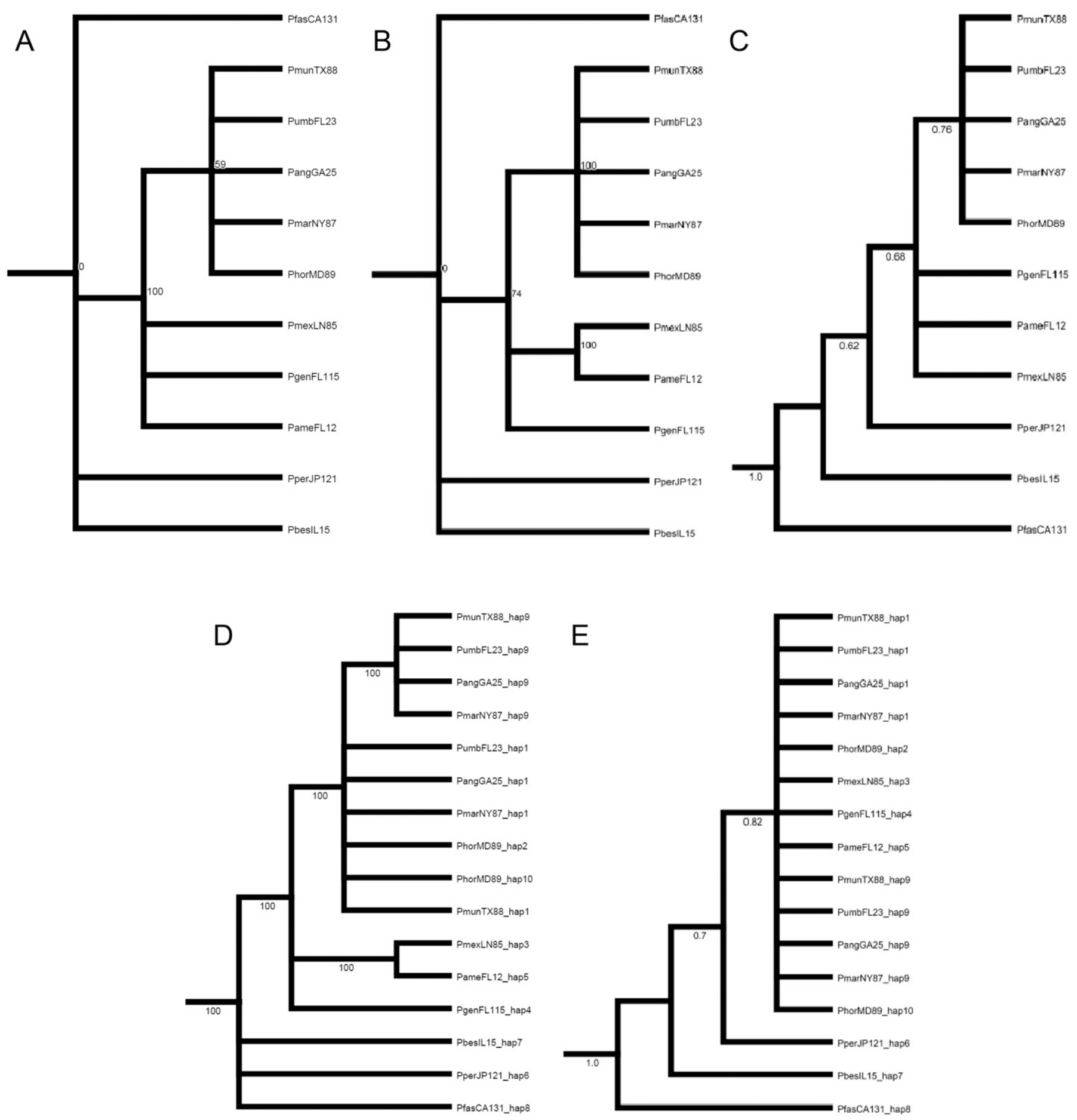

Supplemental Fig. 4. Majority rule consensus trees for $t r n H-p s b A$ chloroplast DNA region sequence data using (A) maximum parsimony, (B) maximum parsimony (including gaps), and (C) maximum likelihood; and sequence data (with haplotype number prefix) using (D) maximum parsimony (including gaps) and (E) maximum likelihood. Bootstrap values greater than 50\% are described above the branches for maximum parsimony tree. Posterior probability values are described above the branches for maximum likehood tree. Trees are rooted with Prunus fasciculata (PfasCA131). Other species in the cladogram are Prunus americana (PameFL12), Prunus angustifolia (PangGA25), Prunus geniculata (PgenFL115), Prunus hortulana (PhorMD89), Prunus maritima (PmarNY87), Prunus mexicana (PmexLN85), Prunus munsoniana (PmunTX88), Prunus persica (PperJP121), Prunus pumila (PbesIL15), and Prunus umbellata (PumbFL23). 

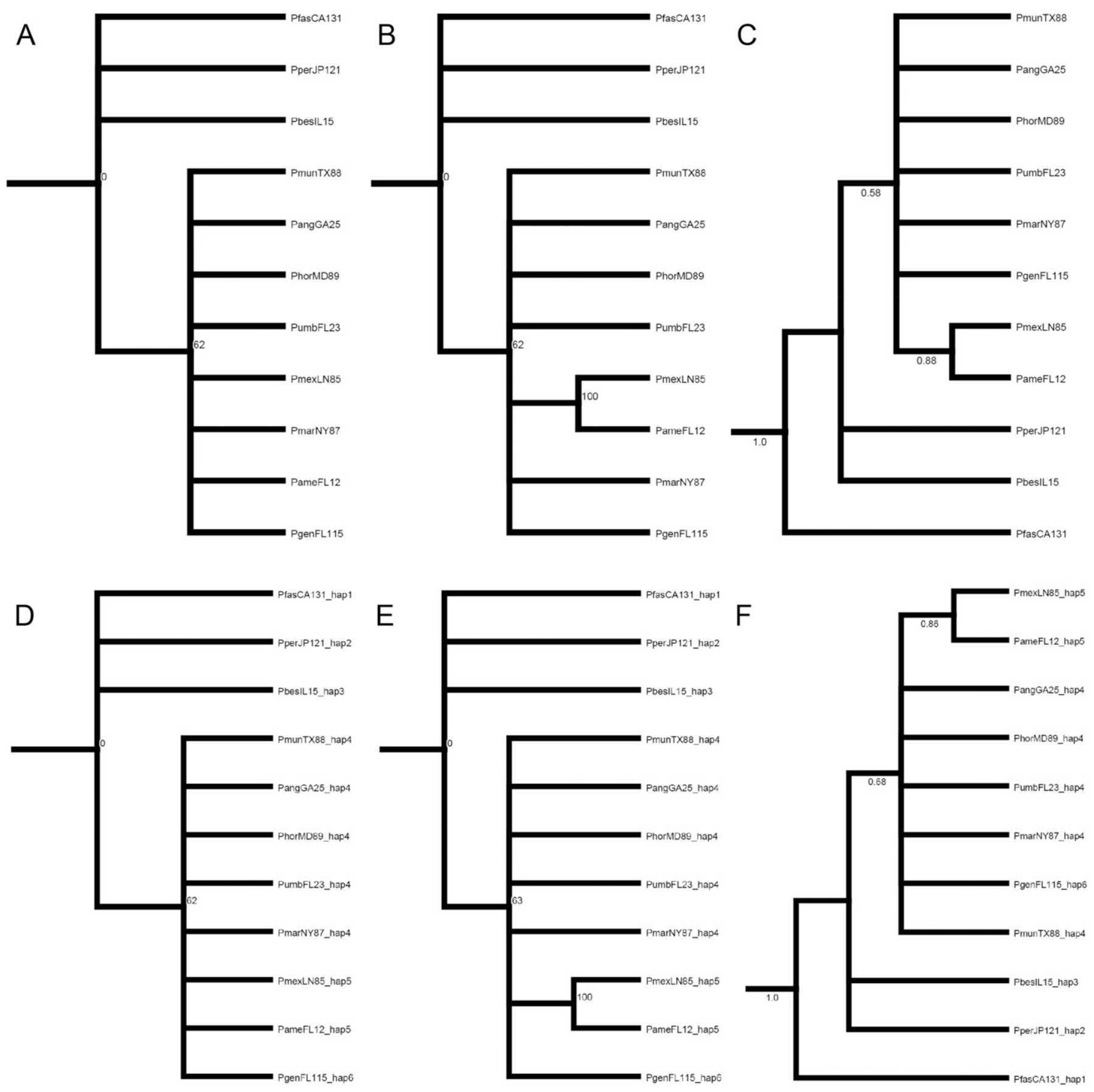

Supplemental Fig. 5. Majority rule consensus trees for $n d h F-r p L 32$ chloroplast DNA region diploid sequence data using (A) maximum parsimony, (B) maximum parsimony (including gaps), and (C) maximum likelihood; and sequence data (with haplotype number prefix) using (D) maximum parsimony, (E) maximum parsimony (including gaps), and (F) maximum likelihood. Bootstrap values greater than $50 \%$ are described above the branches for maximum parsimony tree. Posterior probability values are described above the branches for maximum likehood tree. Trees are rooted with Prunus fasciculata (PfasCA131). Other species in the cladogram are Prunus americana (PameFL12), Prunus angustifolia (PangGA25), Prunus geniculata (PgenFL115), Prunus hortulana (PhorMD89), Prunus maritima (PmarNY87), Prunus mexicana (PmexLN85), Prunus munsoniana (PmunTX88), Prunus persica (PperJP121), Prunus pumila (PbesIL15), and Prunus umbellata (PumbFL23). 

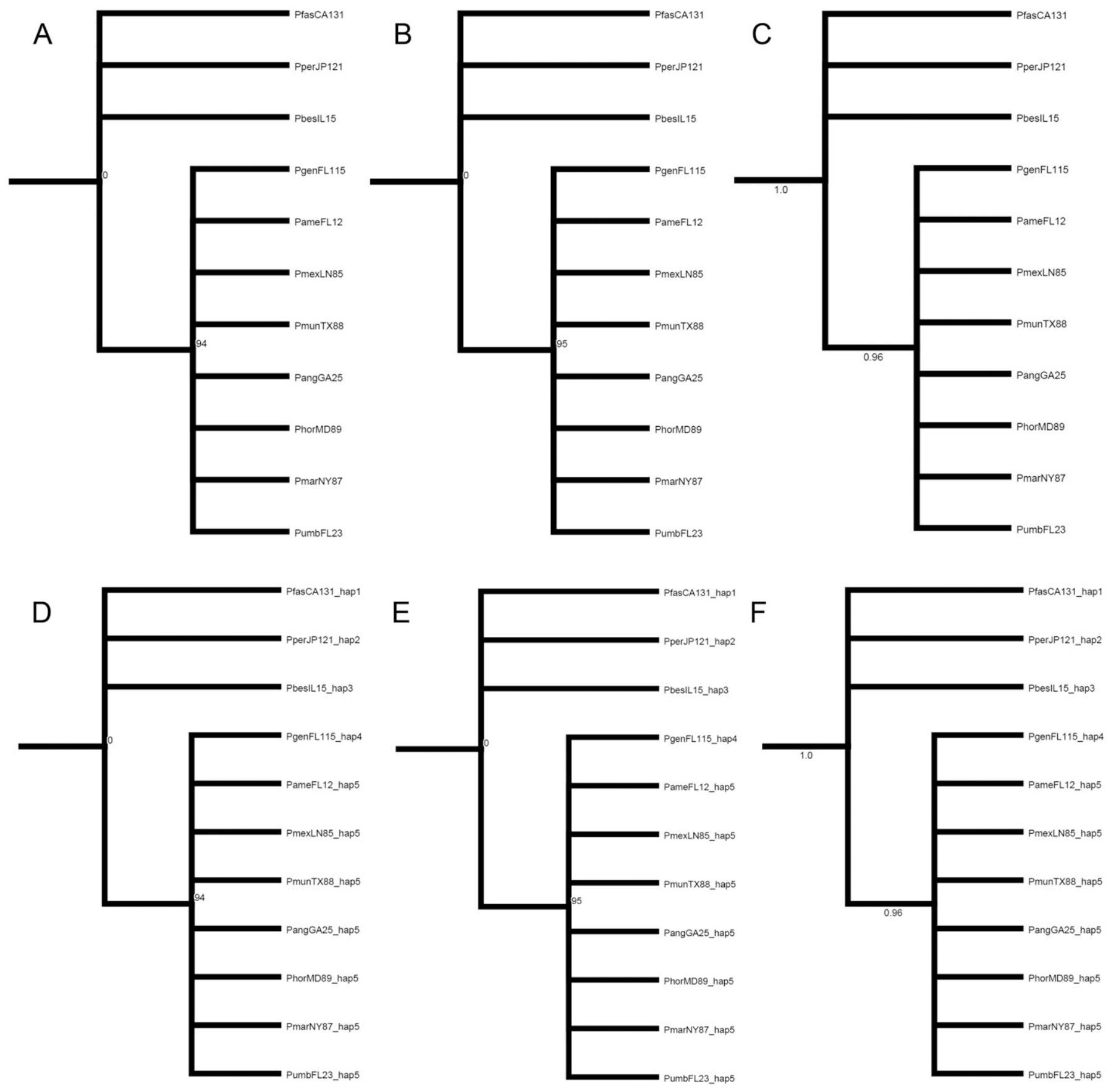

Supplemental Fig. 6. Majority rule consensus trees for $a t p B-r b c L$ chloroplast DNA region diploid sequence data using (A) maximum parsimony, (B) maximum parsimony (including gaps), and (C) maximum likelihood; and sequence data (with haplotype number prefix) using (D) maximum parsimony, (E) maximum parsimony (including gaps), and (F) maximum likelihood. Bootstrap values greater than $50 \%$ are described above the branches for maximum parsimony tree. Posterior probability values are described above the branches for maximum likehood tree. Trees are rooted with Prunus fasciculata (PfasCA131). Other species in the cladogram are Prunus americana (PameFL12), Prunus angustifolia (PangGA25), Prunus geniculata (PgenFL115), Prunus hortulana (PhorMD89), Prunus maritima (PmarNY87), Prunus mexicana (PmexLN85), Prunus munsoniana (PmunTX88), Prunus persica (PperJP121), Prunus pumila (PbesIL15), and Prunus umbellata (PumbFL23). 

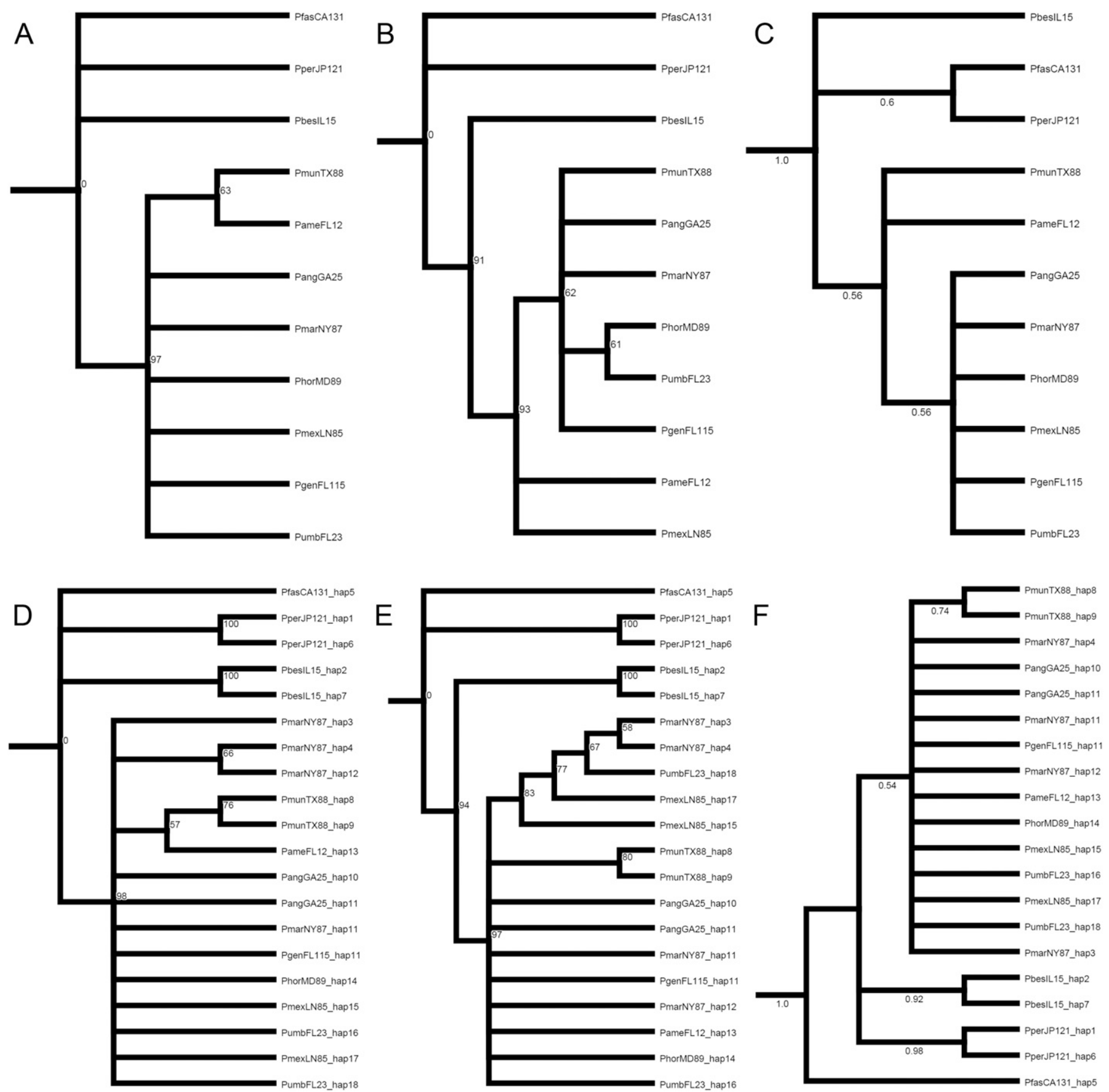

Supplemental Fig. 7. Majority rule consensus trees for internal transcribed spacer diploid sequence data using (A) maximum parsimony, (B) maximum parsimony (including gaps), and (C) maximum likelihood; and haplotypic sequence data using (D) maximum parsimony, (E) maximum parsimony (including gaps), and (F) maximum likelihood. Bootstrap values greater than $50 \%$ are described above the branches for maximum parsimony tree. Posterior probability values are described above the branches for maximum likehood tree. Trees are rooted with Prunus fasciculata (PfasCA131). Other species in the cladogram are Prunus americana (PameFL12), Prunus angustifolia (PangGA25), Prunus geniculata (PgenFl115), Prunus hortulana (PhorMD89), Prunus maritima (PmarNY87), Prunus mexicana (PmexLN85), Prunus munsoniana (PmunTX88), Prunus persica (PperJP121), Prunus pumila (PbesIL15), and Prunus umbellata (PumbFL23). 

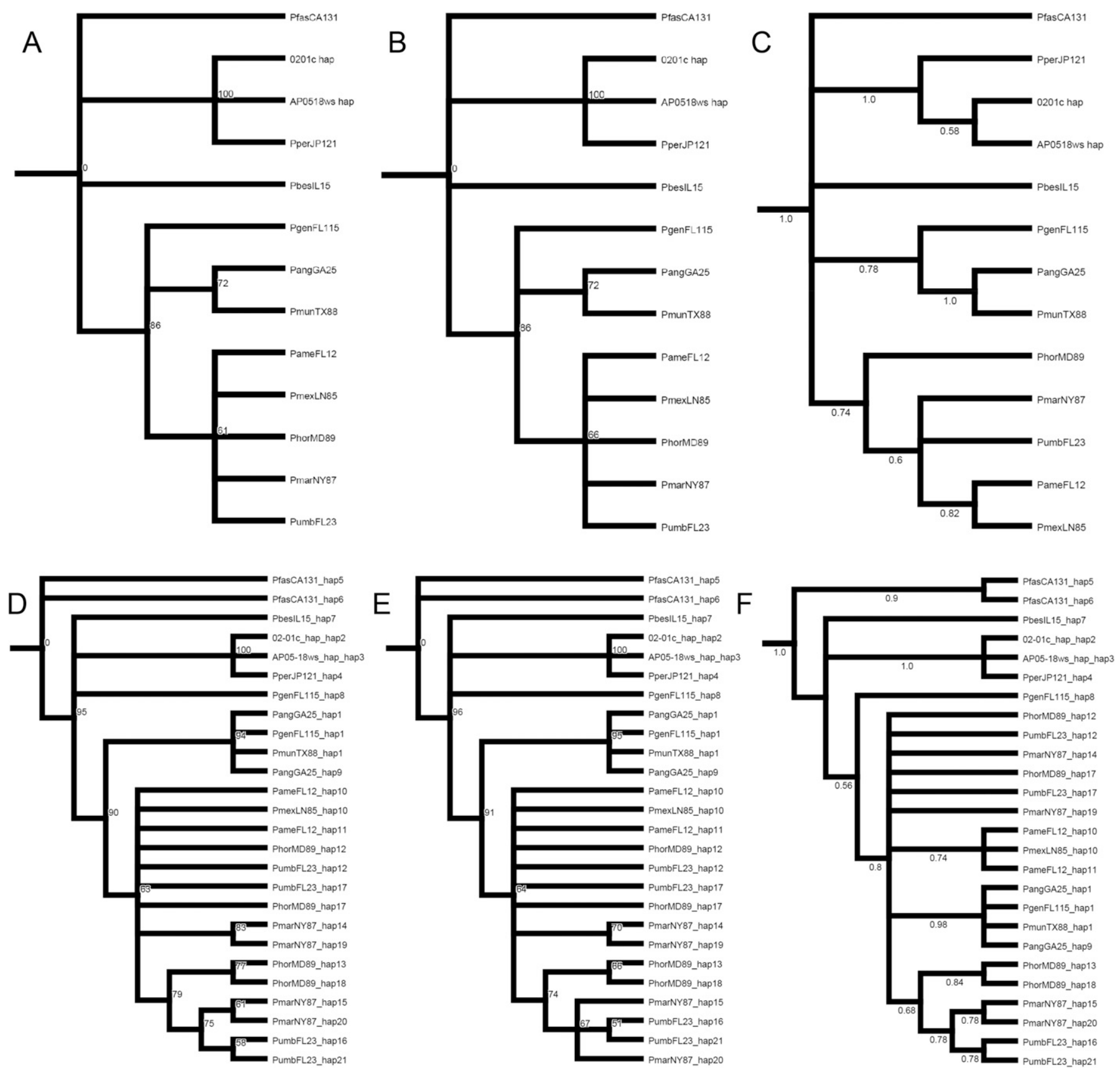

Supplemental Fig. 8. Majority rule consensus trees for PGDH diploid sequence data using (A) maximum parsimony, (B) maximum parsimony (including gaps), and (C) maximum likelihood; and haplotypic sequence data using (D) maximum parsimony, (E) maximum parsimony (including gaps), and (F) maximum likelihood. Bootstrap values greater than $50 \%$ are described above the branches for maximum parsimony tree. Posterior probability values are described above the branches for maximum likehood tree. Trees are rooted with Prunus fasciculata (PfasCA131). Other species in the cladogram are Prunus americana (PameFL12), Prunus angustifolia (PangGA25), Prunus geniculata (PgenFL115), Prunus hortulana (PhorMD89), Prunus maritima (PmarNY87), Prunus mexicana (PmexLN85), Prunus munsoniana (PmunTX88), Prunus persica (PperJP121, 0201c_hap, AP0518ws_hap), Prunus pumila (PbesIL15), and Prunus umbellata (PumbFL23). 

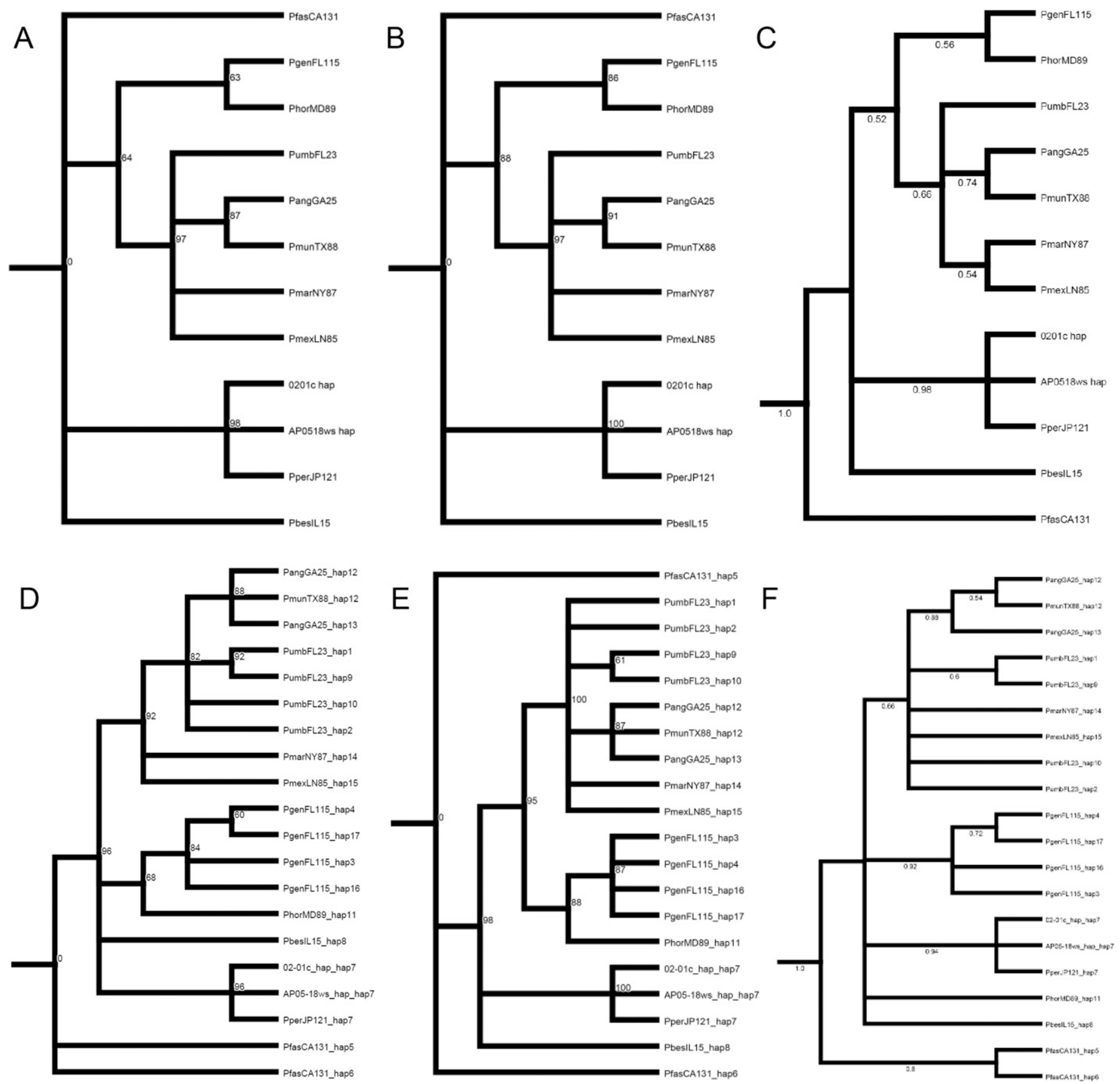

Supplemental Fig. 9. Majority rule consensus trees for PGI diploid sequence data using (A) maximum parsimony, (B) maximum parsimony (including gaps), and (C) maximum likelihood; and haplotypic sequence data using (D) maximum parsimony, (E) maximum parsimony (including gaps), and (F) maximum likelihood. Bootstrap values greater than $50 \%$ are described above the branches for maximum parsimony tree. Posterior probability values are described above the branches for maximum likehood tree. Trees are rooted with Prunus fasciculata (PfasCA131). Other species in the cladogram are Prunus americana (PameFL12), Prunus angustifolia (PangGA25), Prunus geniculata (PgenFL115), Prunus hortulana (PhorMD89), Prunus maritima (PmarNY87), Prunus mexicana (PmexLN85), Prunus munsoniana (PmunTX88), Prunus persica (PperJP121, 0201c_hap, AP0518ws_hap), Prunus pumila (PbesIL15), and Prunus umbellata (PumbFL23). 

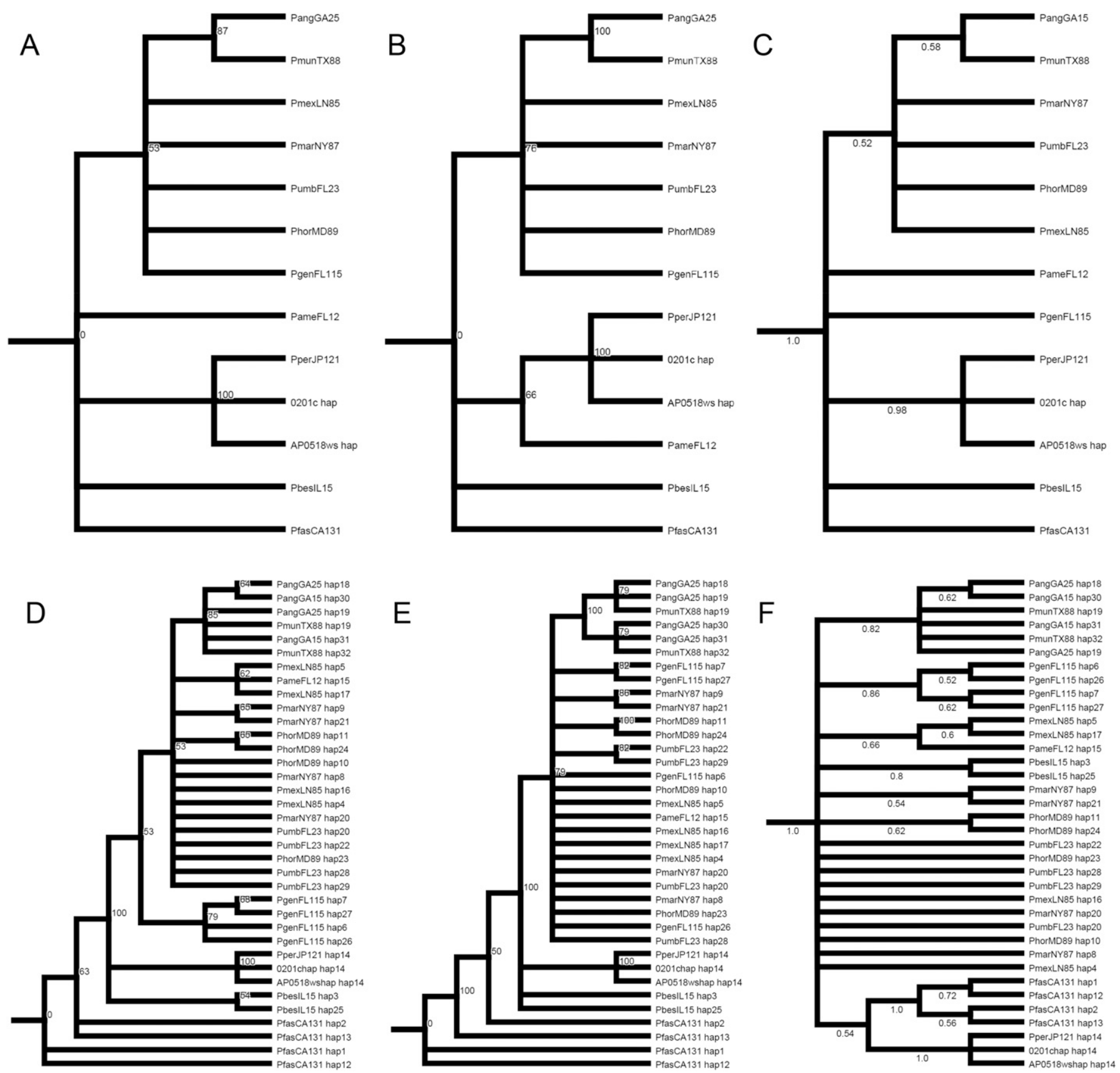

Supplemental Fig. 10. Majority rule consensus trees for S6PDH diploid sequence data using (A) maximum parsimony, (B) maximum parsimony (including gaps), and (C) maximum likelihood; and haplotypic sequence data using (D) maximum parsimony, (E) maximum parsimony (including gaps), and (F) maximum likelihood. Bootstrap values greater than $50 \%$ are described above the branches for maximum parsimony tree. Posterior probability values are described above the branches for maximum likehood tree. Trees are rooted with Prunus fasciculata (PfasCA131). Other species in the cladogram are Prunus americana (PameFL12), Prunus angustifolia (PangGA25), Prunus geniculata (PgenFL115), Prunus hortulana (PhorMD89), Prunus maritima (PmarNY87), Prunus mexicana (PmexLN85), Prunus munsoniana (PmunTX88), Prunus persica (PperJP121, 0201c_hap, AP0518ws_hap), Prunus pumila (PbesIL15), and Prunus umbellata (PumbFL23). 

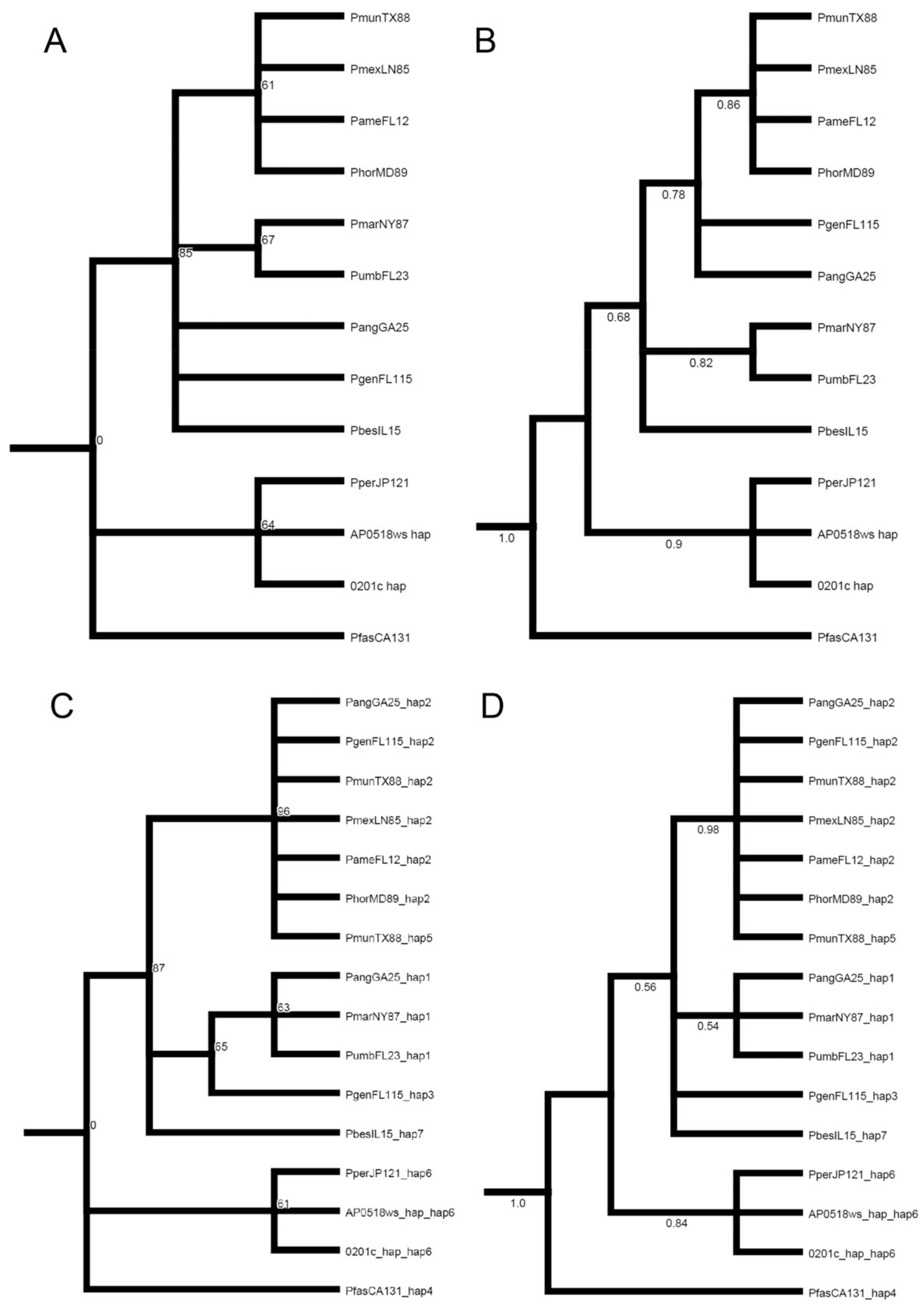

Supplemental Fig. 11. Majority rule consensus trees for AXR1 diploid sequence data using (A) maximum parsimony and (B) maximum likelihood; and haplotypic sequence data using $(\mathbf{C})$ maximum parsimony and (D) maximum likelihood. Bootstrap values greater than $50 \%$ are described above the branches for maximum parsimony tree. Posterior probability values are described above the branches for maximum likehood tree. Trees are rooted with Prunus fasciculata (PfasCA131). Other species in the cladogram are Prunus americana (PameFL12), Prunus angustifolia (PangGA25), Prunus geniculata (PgenFL115), Prunus hortulana (PhorMD89), Prunus maritima (PmarNY87), Prunus mexicana (PmexLN85), Prunus munsoniana (PmunTX88), Prunus persica (PperJP121, 0201c_hap, AP0518ws_hap), Prunus pumila (PbesIL15), and Prunus umbellata (PumbFL23). 

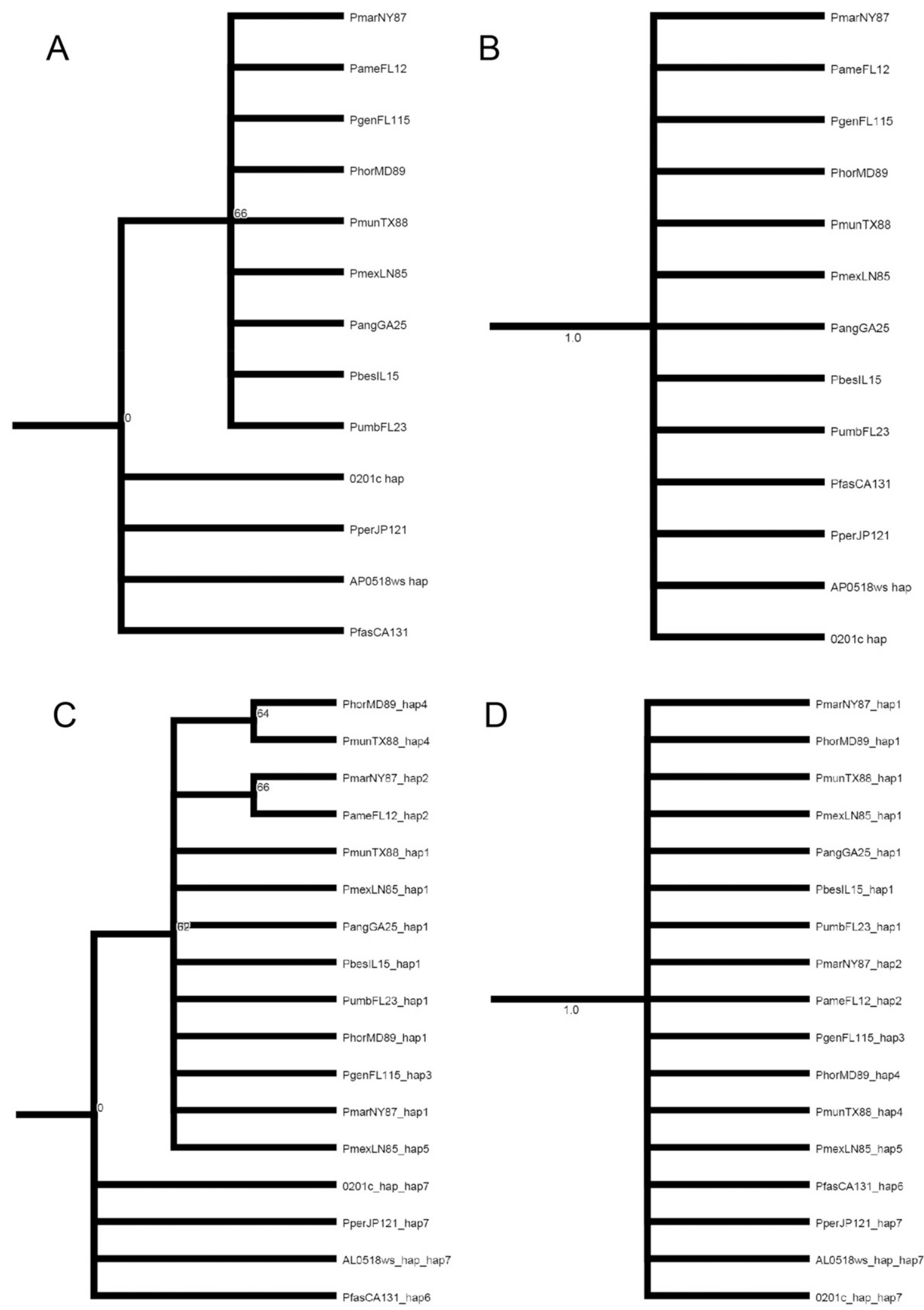

Supplemental Fig. 12. Majority rule consensus trees for BRC1 diploid sequence data using (A) maximum parsimony and (B) maximum likelihood; and haplotypic sequence data using $(\mathbf{C})$ maximum parsimony and (D) maximum likelihood. Bootstrap values greater than $50 \%$ are described above the branches for maximum parsimony tree. Posterior probability values are described above the branches for maximum likehood tree. Trees are rooted with Prunus fasciculata (PfasCA131). Other species in the cladogram are Prunus americana (PameFL12), Prunus angustifolia (PangGA25), Prunus geniculata (PgenFL115), Prunus hortulana (PhorMD89), Prunus maritima (PmarNY87), Prunus mexicana (PmexLN85), Prunus munsoniana (PmunTX88), Prunus persica (PperJP121, 0201c_hap, AP0518ws_hap), Prunus pumila (PbesIL15), and Prunus umbellata (PumbFL23). 

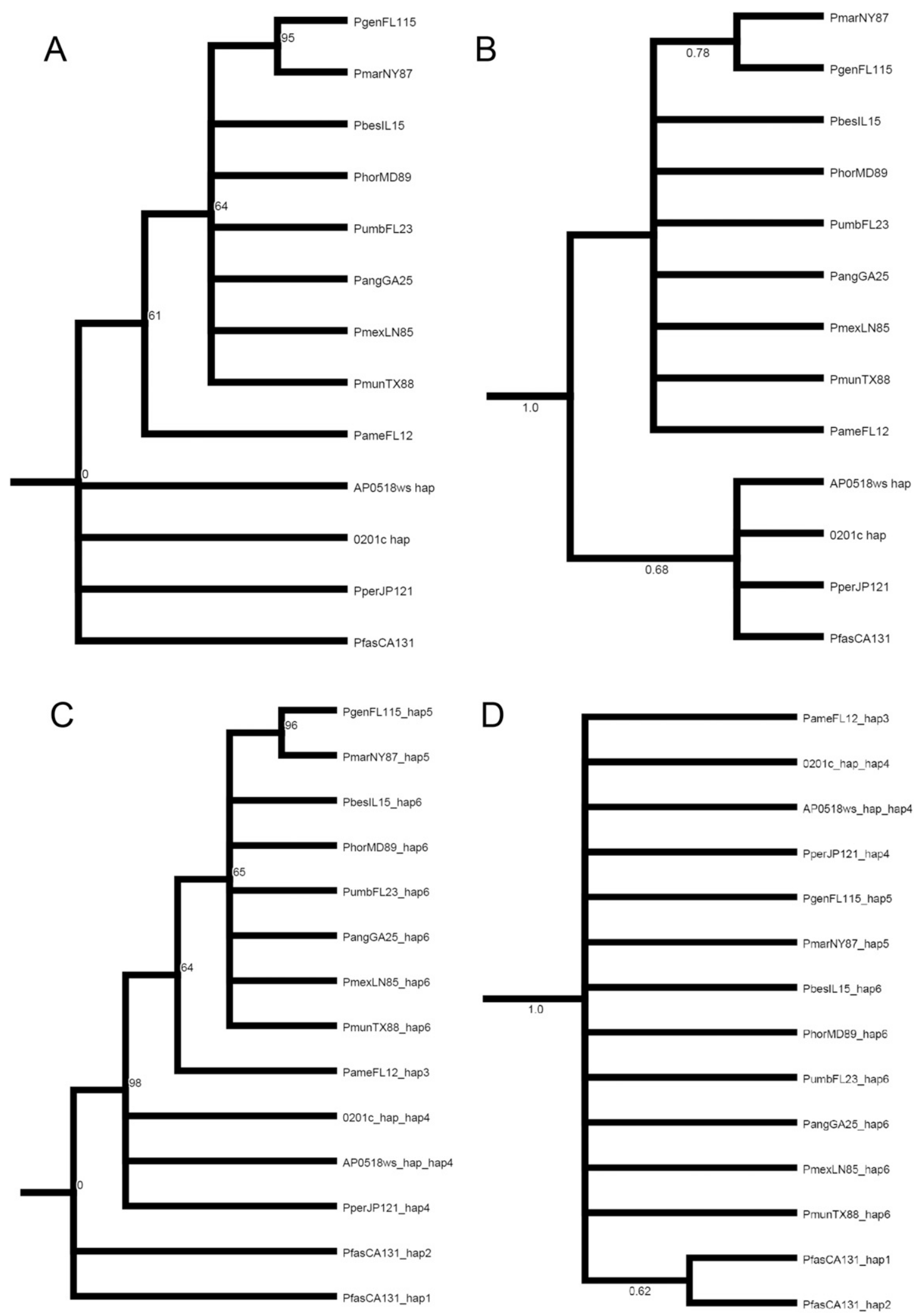

Supplemental Fig. 13. Majority rule consensus trees for BRC2 diploid sequence data using (A) maximum parsimony and (B) maximum likelihood; and haplotypic sequence data using $(\mathbf{C})$ maximum parsimony and (D) maximum likelihood. Bootstrap values greater than $50 \%$ are described above the branches for maximum parsimony tree. Posterior probability values are described above the branches for maximum likehood tree. Trees are rooted with Prunus fasciculata (PfasCA131). Other species in the cladogram are Prunus americana (PameFL12), Prunus angustifolia (PangGA25), Prunus geniculata (PgenFL115), Prunus hortulana (PhorMD89), Prunus maritima (PmarNY87), Prunus mexicana (PmexLN85), Prunus munsoniana (PmunTX88), Prunus persica (PperJP121, 0201c_hap, AP0518ws_hap), Prunus pumila (PbesIL15), and Prunus umbellata (PumbFL23). 

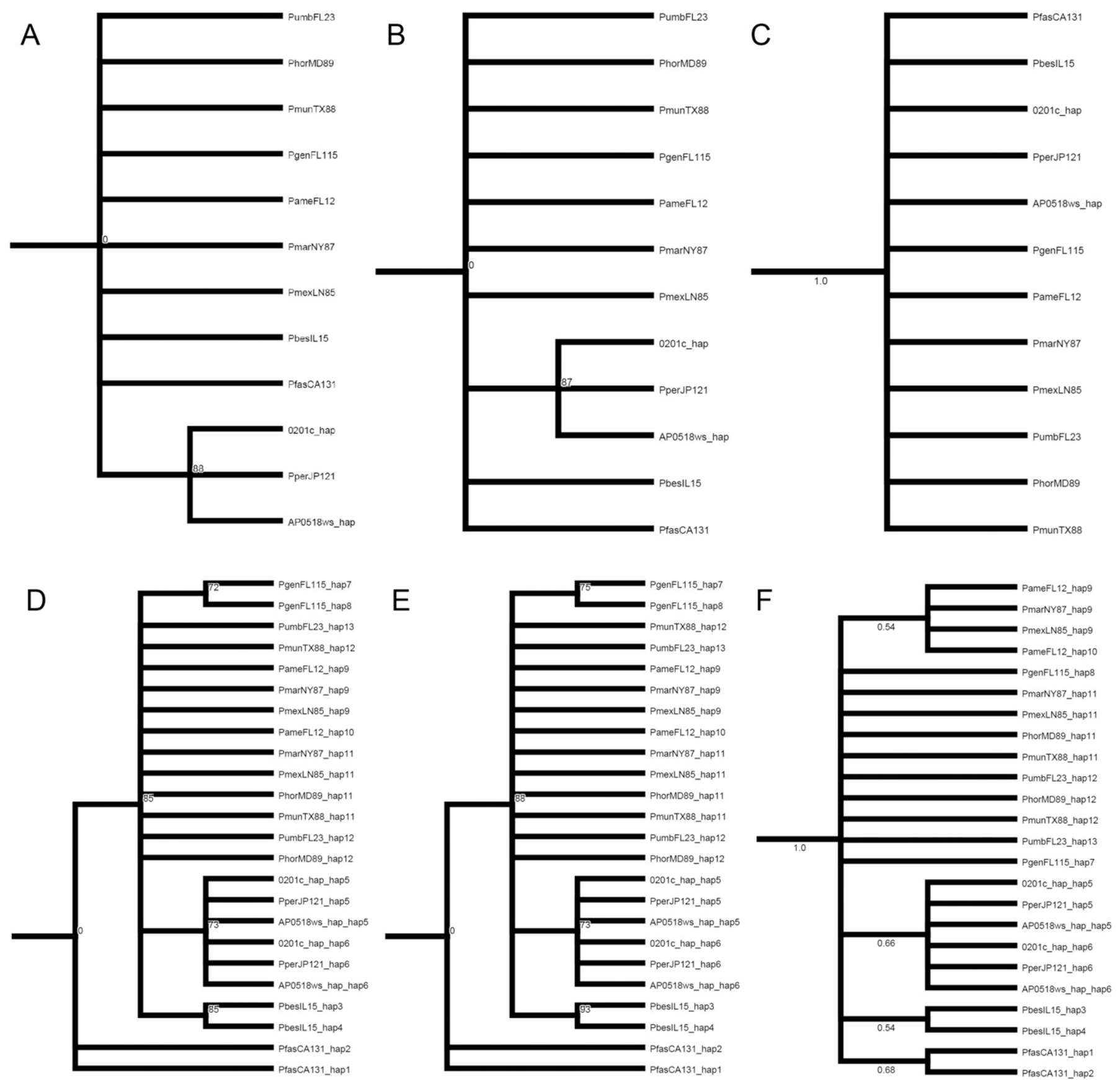

Supplemental Fig. 14. Majority rule consensus trees for CUC1A diploid sequence data using (A) maximum parsimony, (B) maximum parsimony (including gaps), and $(\mathbf{C})$ maximum likelihood; and haplotypic sequence data using (D) maximum parsimony, (E) maximum parsimony (including gaps), and (F) maximum likelihood. Bootstrap values greater than $50 \%$ are described above the branches for maximum parsimony tree. Posterior probability values are described above the branches for maximum likehood tree. Trees are rooted with Prunus fasciculata (PfasCA131). Other species in the cladogram are Prunus americana (PameFL12), Prunus angustifolia (PangGA25), Prunus geniculata (PgenFL115), Prunus hortulana (PhorMD89), Prunus maritima (PmarNY87), Prunus mexicana (PmexLN85), Prunus munsoniana (PmunTX88), Prunus persica (PperJP121, 0201c_hap, AP0518ws_hap), Prunus pumila (PbesIL15), and Prunus umbellata (PumbFL23). 

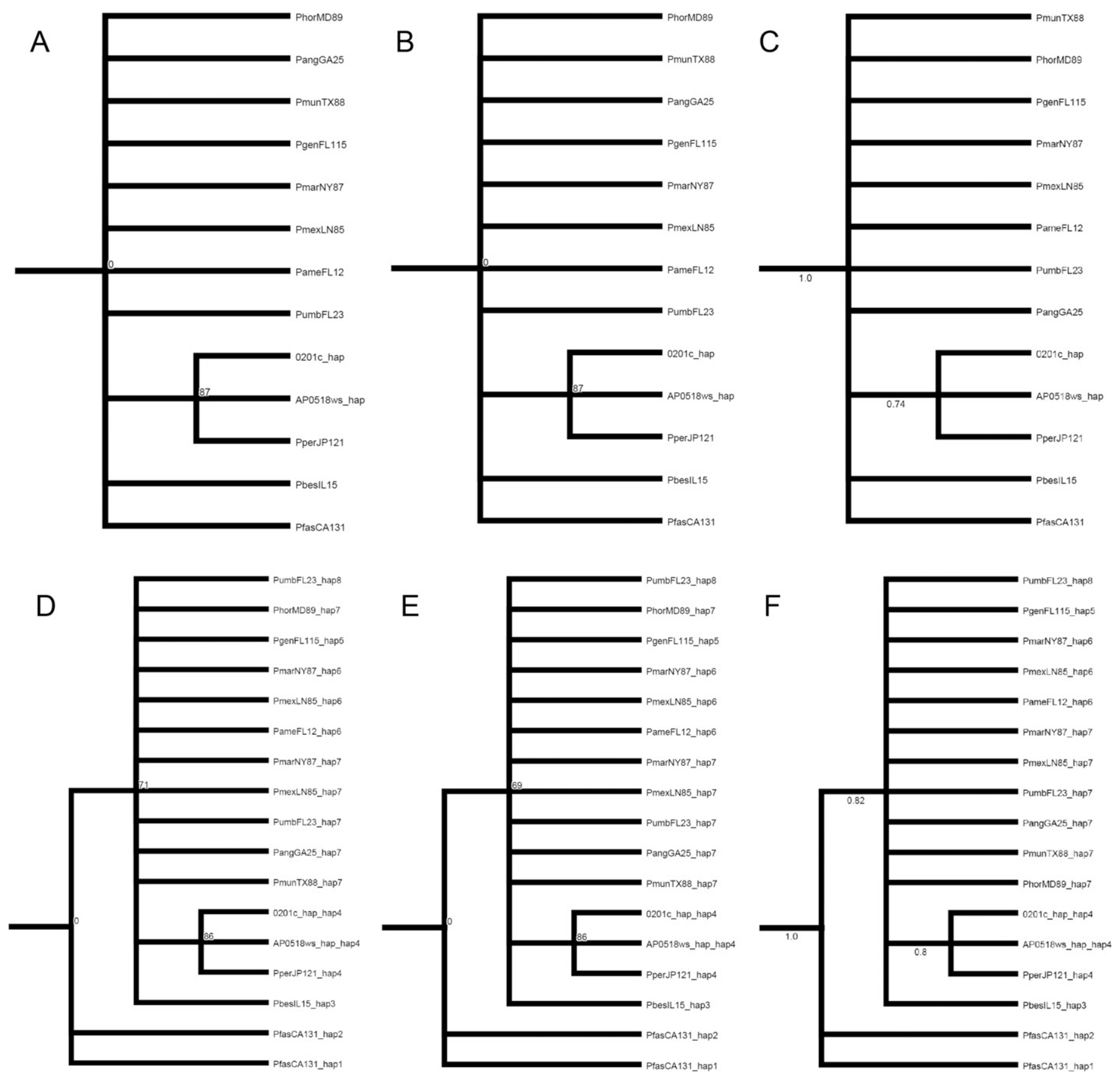

Supplemental Fig. 15. Majority rule consensus trees for CUC1B diploid sequence data using (A) maximum parsimony, (B) maximum parsimony (including gaps), and (C) maximum likelihood; and haplotypic sequence data using (D) maximum parsimony, (E) maximum parsimony (including gaps), and (F) maximum likelihood. Bootstrap values greater than $50 \%$ are described above the branches for maximum parsimony tree. Posterior probability values are described above the branches for maximum likehood tree. Trees are rooted with Prunus fasciculata (PfasCA131). Other species in the cladogram are Prunus americana (PameFL12), Prunus angustifolia (PangGA25), Prunus geniculata (PgenFL115), Prunus hortulana (PhorMD89), Prunus maritima (PmarNY87), Prunus mexicana (PmexLN85), Prunus munsoniana (PmunTX88), Prunus persica (PperJP121, 0201c_hap, AP0518ws_hap), Prunus pumila (PbesIL15), and Prunus umbellata (PumbFL23). 

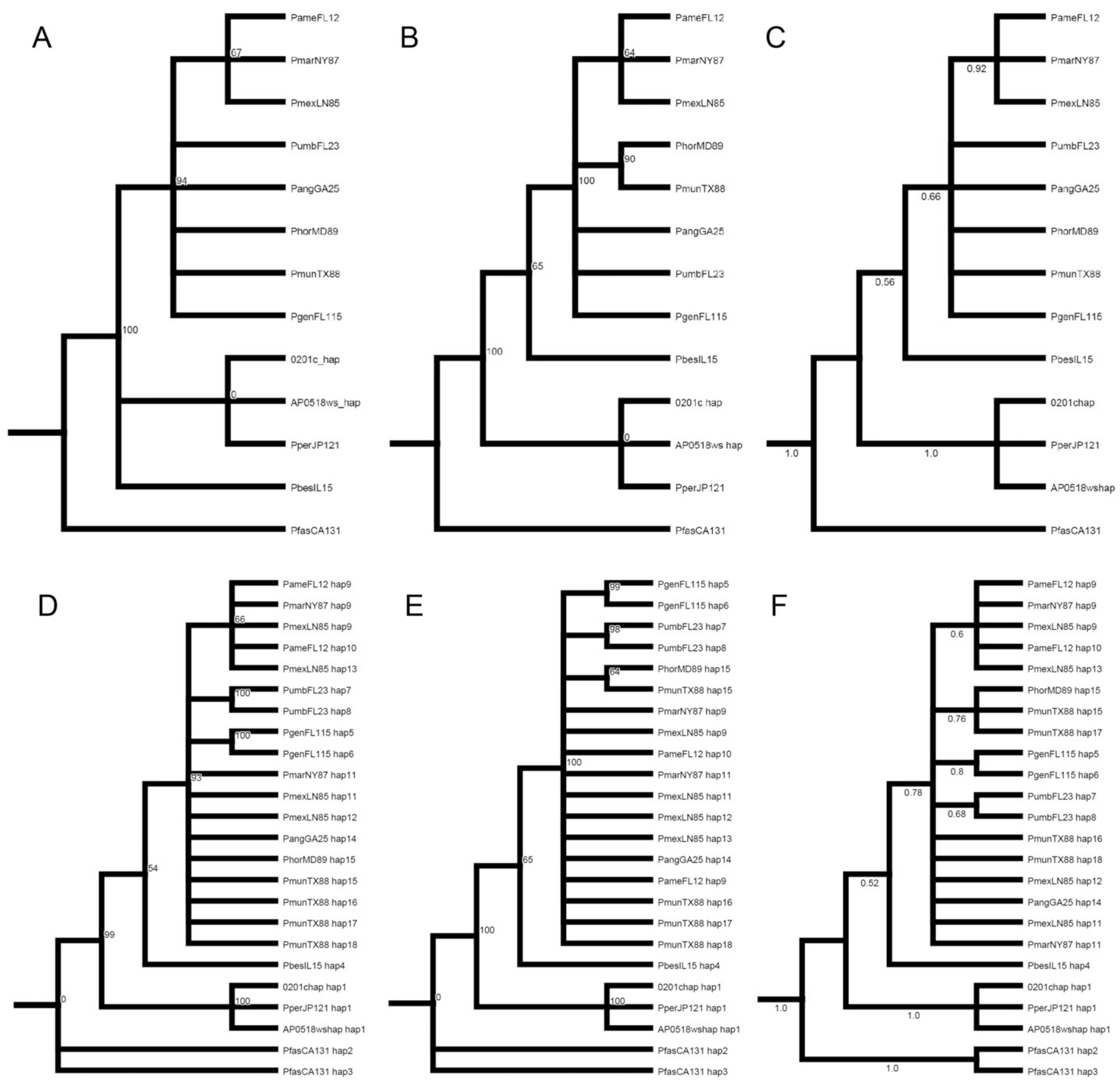

Supplemental Fig. 16. Majority rule consensus trees for CUC2 diploid sequence data using (A) maximum parsimony, (B) maximum parsimony (including gaps), and $(\mathbf{C})$ maximum likelihood; and haplotypic sequence data using $(\mathbf{D})$ maximum parsimony, (E) maximum parsimony (including gaps), and (F) maximum likelihood. Bootstrap values greater than $50 \%$ are described above the branches for maximum parsimony tree. Posterior probability values are described above the branches for maximum likehood tree. Trees are rooted with Prunus fasciculata (PfasCA131). Other species in the cladogram are Prunus americana (PameFL12), Prunus angustifolia (PangGA25), Prunus geniculata (PgenFL115), Prunus hortulana (PhorMD89), Prunus maritima (PmarNY87), Prunus mexicana (PmexLN85), Prunus munsoniana (PmunTX88), Prunus persica (PperJP121, 0201c_hap, AP0518ws_hap), Prunus pumila (PbesIL15), and Prunus umbellata (PumbFL23). 

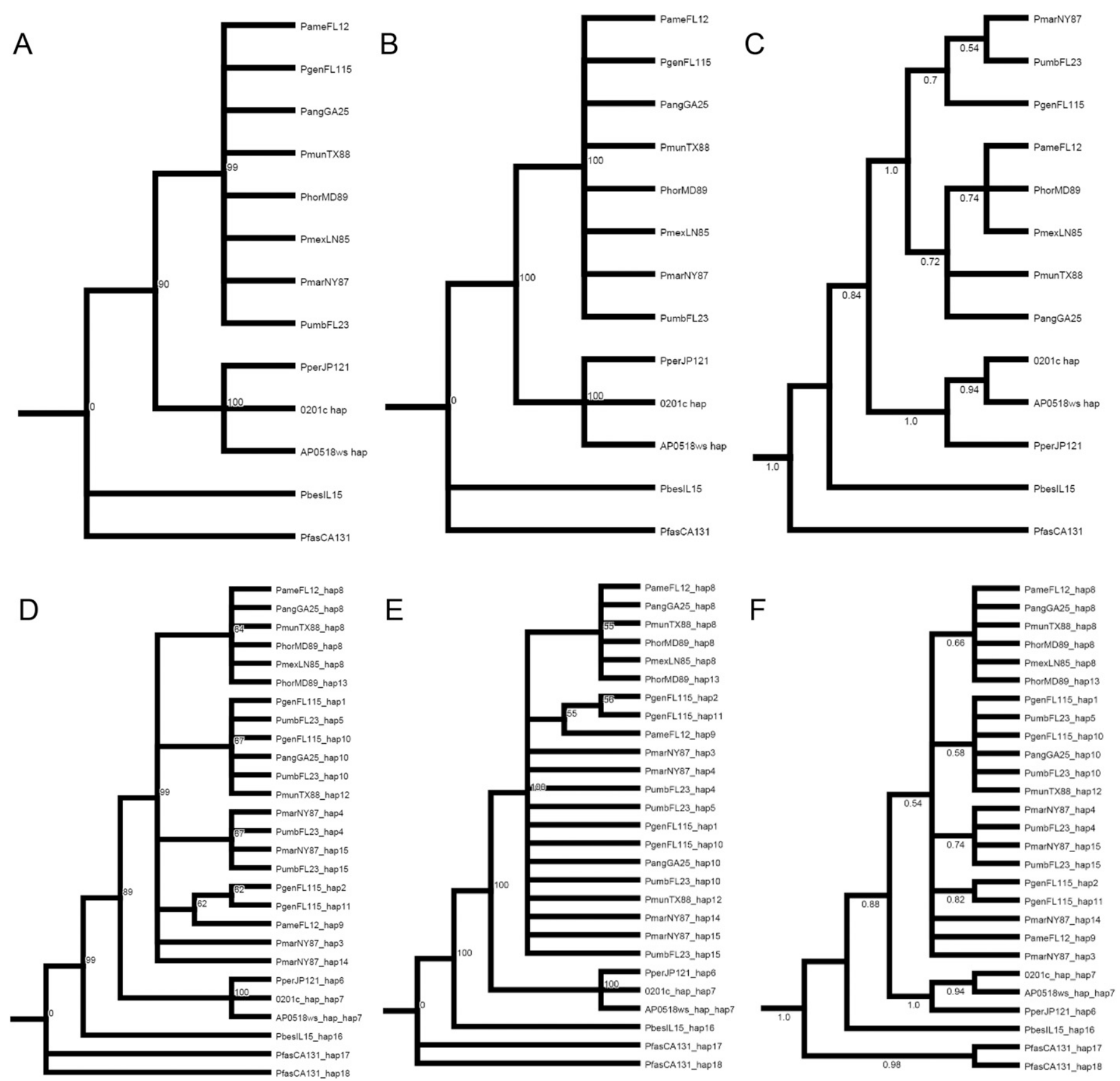

Supplemental Fig. 17. Majority rule consensus trees for CUC3 diploid sequence data using (A) maximum parsimony, (B) maximum parsimony (including gaps), and (C) maximum likelihood; and haplotypic sequence data using (D) maximum parsimony, (E) maximum parsimony (including gaps), and (F) maximum likelihood. Bootstrap values greater than $50 \%$ are described above the branches for maximum parsimony tree. Posterior probability values are described above the branches for maximum likehood tree. Trees are rooted with Prunus fasciculata (PfasCA131). Other species in the cladogram are Prunus americana (PameFL12), Prunus angustifolia (PangGA25), Prunus geniculata (PgenFl115), Prunus hortulana (PhorMD89), Prunus maritima (PmarNY87), Prunus mexicana (PmexLN85), Prunus munsoniana (PmunTX88), Prunus persica (PperJP121, 0201c_hap, AP0518ws_hap), Prunus pumila (PbesIL15), and Prunus umbellata (PumbFL23). 

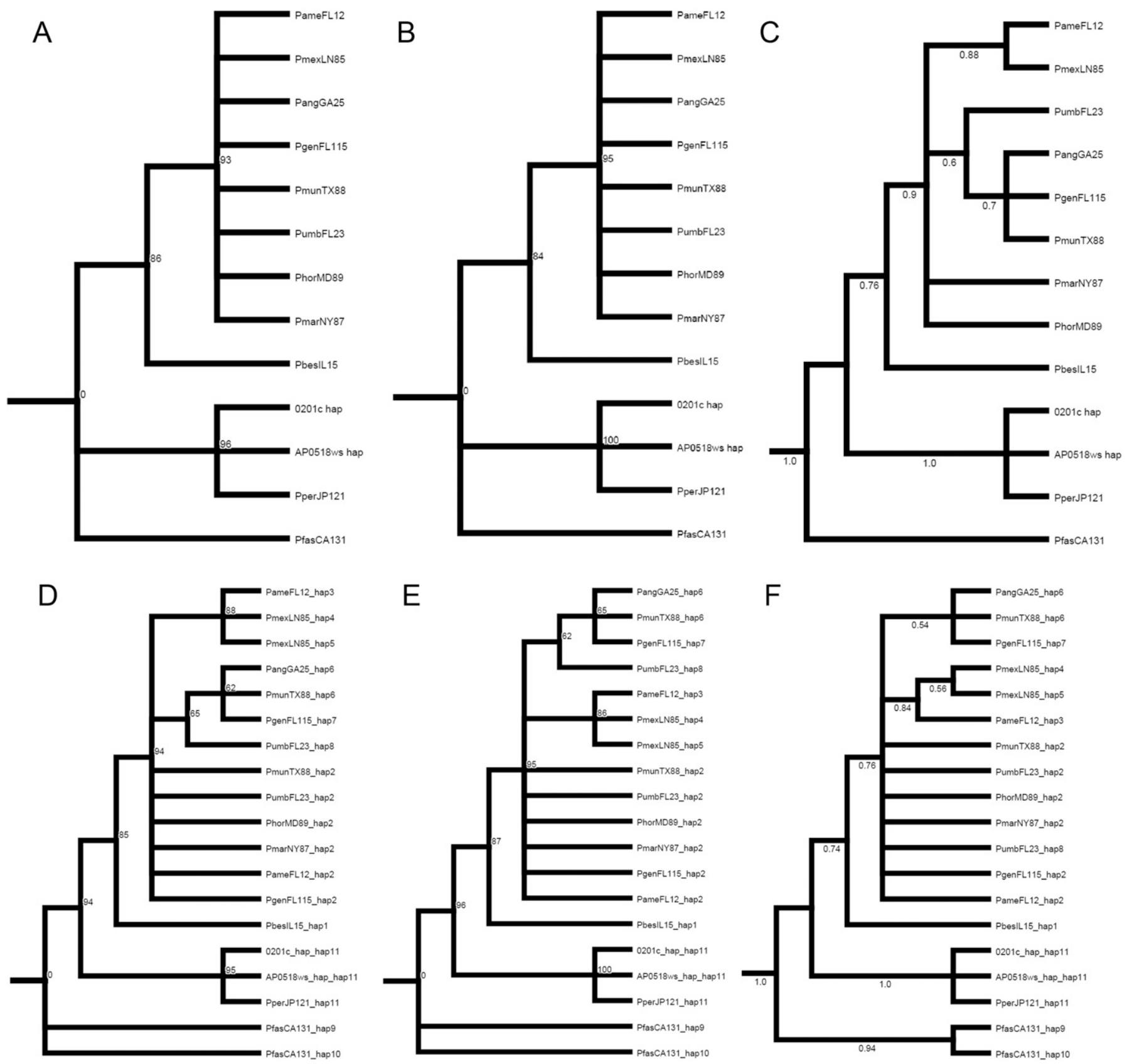

Supplemental Fig. 18. Majority rule consensus trees for LAS diploid sequence data using (A) maximum parsimony, (B) maximum parsimony (including gaps), and (C) maximum likelihood; and haplotypic sequence data using (D) maximum parsimony, (E) maximum parsimony (including gaps), and (F) maximum likelihood. Bootstrap values greater than $50 \%$ are described above the branches for maximum parsimony tree. Posterior probability values are described above the branches for maximum likehood tree. Trees are rooted with Prunus fasciculata (PfasCA131). Other species in the cladogram are Prunus americana (PameFL12), Prunus angustifolia (PangGA25), Prunus geniculata (PgenFL115), Prunus hortulana (PhorMD89), Prunus maritima (PmarNY87), Prunus mexicana (PmexLN85), Prunus munsoniana (PmunTX88), Prunus persica (PperJP121, 0201c_hap, AP0518ws_hap), Prunus pumila (PbesIL15), and Prunus umbellata (PumbFL23). 

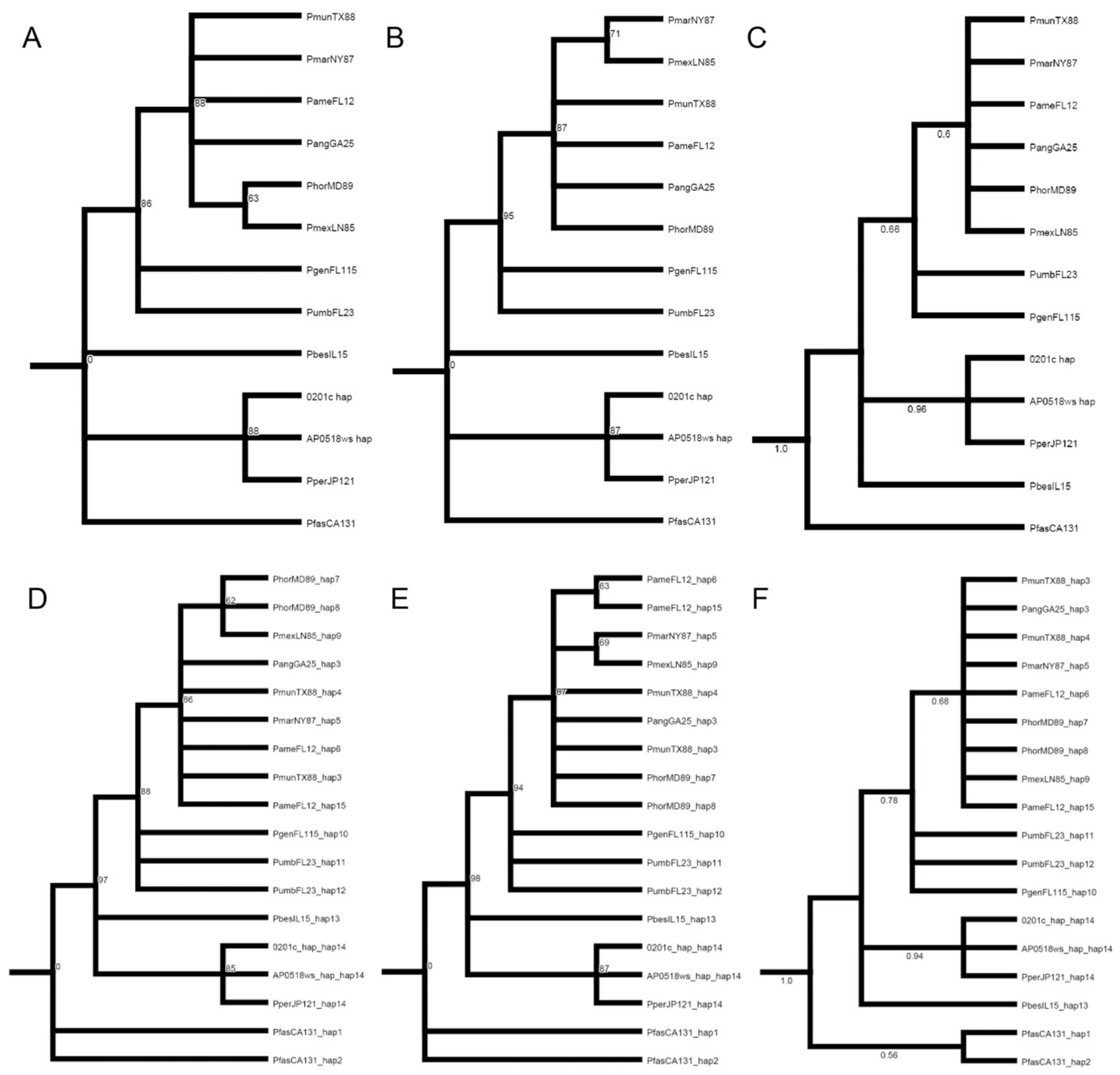

Supplemental Fig. 19. Majority rule consensus trees for MAX1A diploid sequence data using (A) maximum parsimony, (B) maximum parsimony (including gaps), and $(\mathbf{C})$ maximum likelihood; and haplotypic sequence data using (D) maximum parsimony, (E) maximum parsimony (including gaps), and (F) maximum likelihood. Bootstrap values greater than $50 \%$ are described above the branches for maximum parsimony tree. Posterior probability values are described above the branches for maximum likehood tree. Trees are rooted with Prunus fasciculata (PfasCA131). Other species in the cladogram are Prunus americana (PameFL12), Prunus angustifolia (PangGA25), Prunus geniculata (PgenFL115), Prunus hortulana (PhorMD89), Prunus maritima (PmarNY87), Prunus mexicana (PmexLN85), Prunus munsoniana (PmunTX88), Prunus persica (PperJP121, 0201c_hap, AP0518ws_hap), Prunus pumila (PbesIL15), and Prunus umbellata (PumbFL23). 

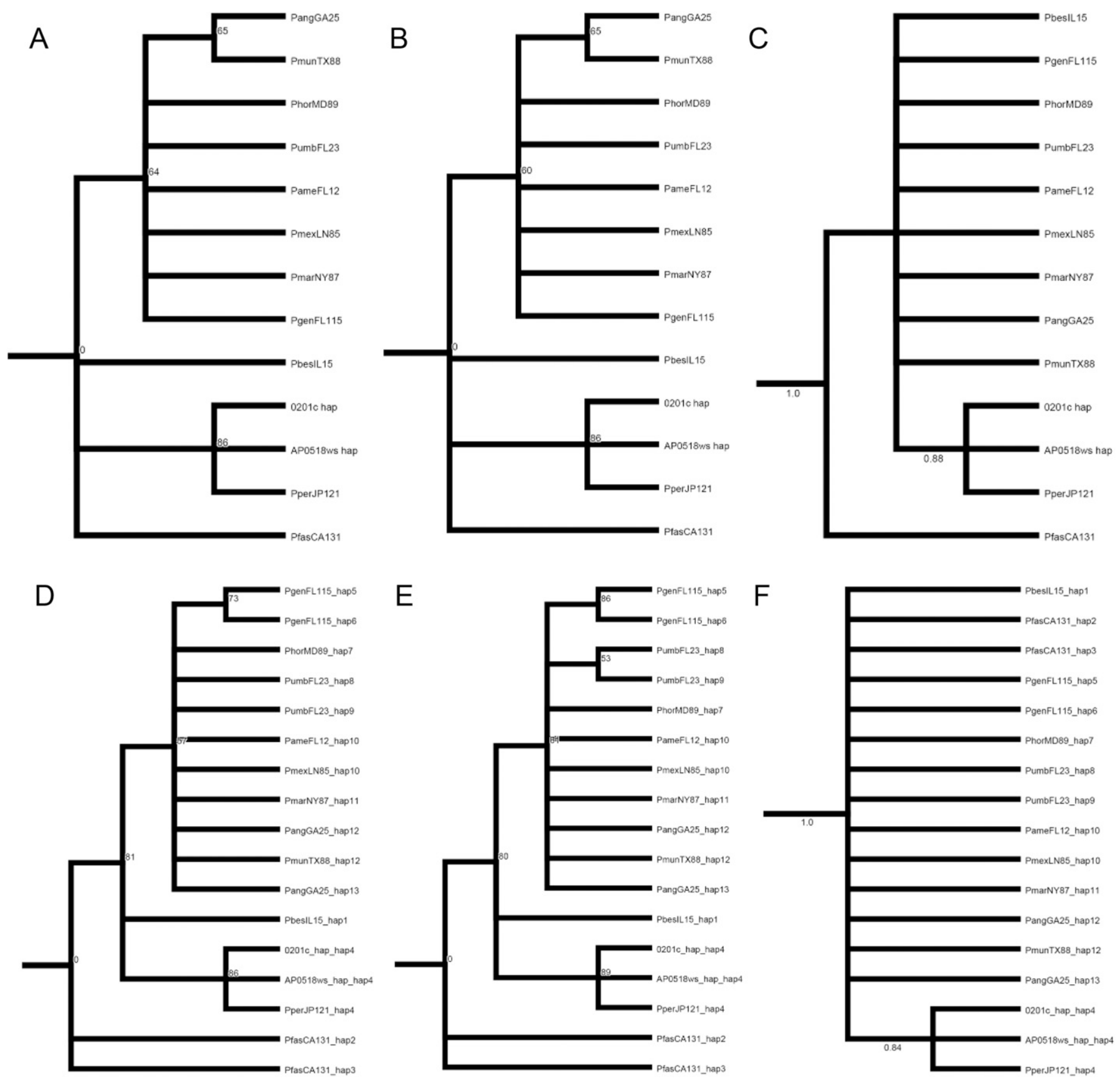

Supplemental Fig. 20. Majority rule consensus trees for MAX1B diploid sequence data using (A) maximum parsimony, (B) maximum parsimony (including gaps), and $(\mathbf{C})$ maximum likelihood; and haplotypic sequence data using (D) maximum parsimony, (E) maximum parsimony (including gaps), and (F) maximum likelihood. Bootstrap values greater than $50 \%$ are described above the branches for maximum parsimony tree. Posterior probability values are described above the branches for maximum likehood tree. Trees are rooted with Prunus fasciculata (PfasCA131). Other species in the cladogram are Prunus americana (PameFL12), Prunus angustifolia (PangGA25), Prunus geniculata (PgenFL115), Prunus hortulana (PhorMD89), Prunus maritima (PmarNY87), Prunus mexicana (PmexLN85), Prunus munsoniana (PmunTX88), Prunus persica (PperJP121, 0201c_hap, AP0518ws_hap), Prunus pumila (PbesIL15), and Prunus umbellata (PumbFL23) 

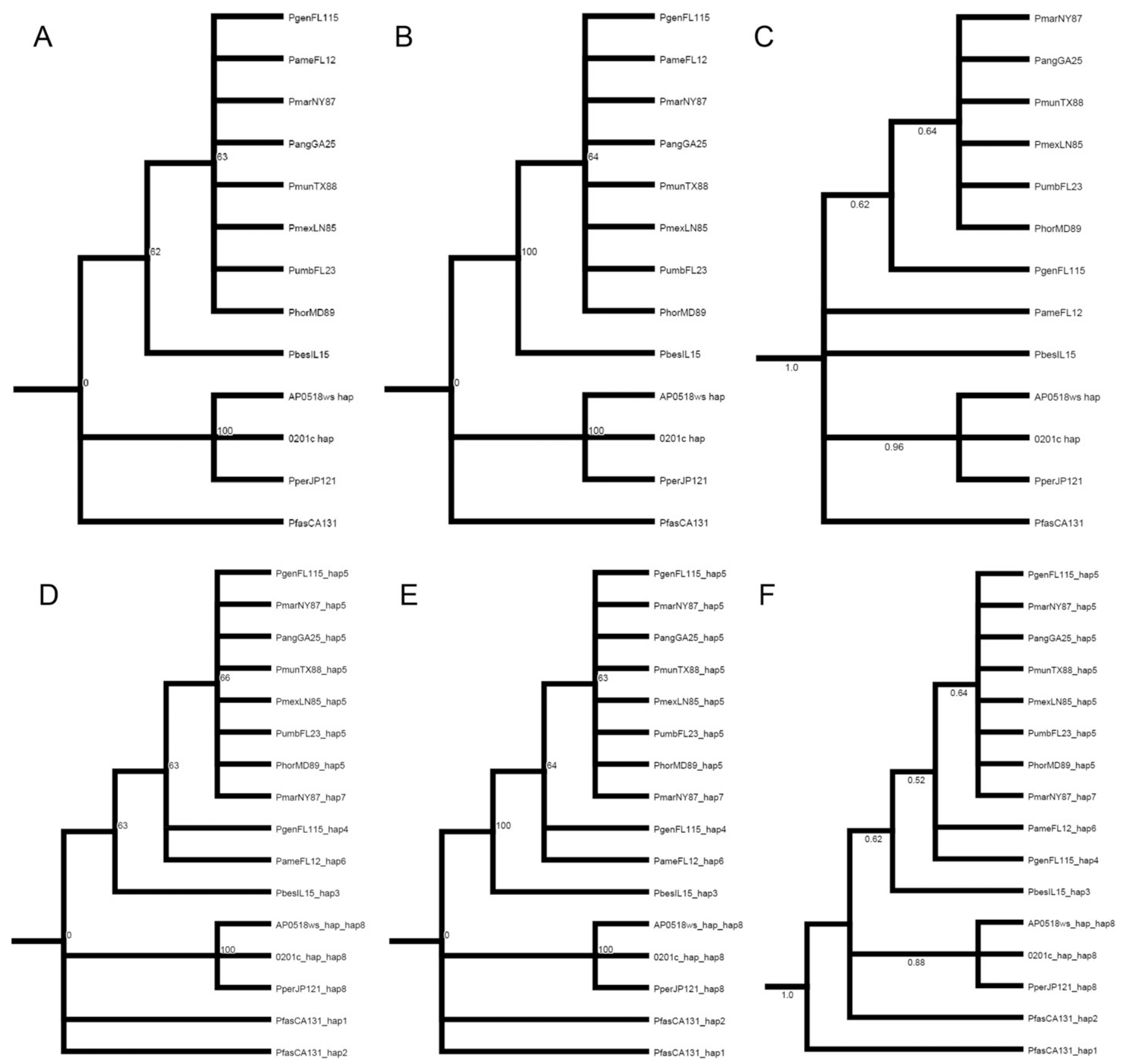

Supplemental Fig. 21. Majority rule consensus trees for MAX2 diploid sequence data using (A) maximum parsimony, (B) maximum parsimony (including gaps), and (C) maximum likelihood; and haplotypic sequence data using (D) maximum parsimony, (E) maximum parsimony (including gaps), and (F) maximum likelihood. Bootstrap values greater than $50 \%$ are described above the branches for maximum parsimony tree. Posterior probability values are described above the branches for maximum likehood tree. Trees are rooted with Prunus fasciculata (PfasCA131). Other species in the cladogram are Prunus americana (PameFL12), Prunus angustifolia (PangGA25), Prunus geniculata (PgenFL115), Prunus hortulana (PhorMD89), Prunus maritima (PmarNY87), Prunus mexicana (PmexLN85), Prunus munsoniana (PmunTX88), Prunus persica (PperJP121, 0201c_hap, AP0518ws_hap), Prunus pumila (PbesIL15), and Prunus umbellata (PumbFL23). 

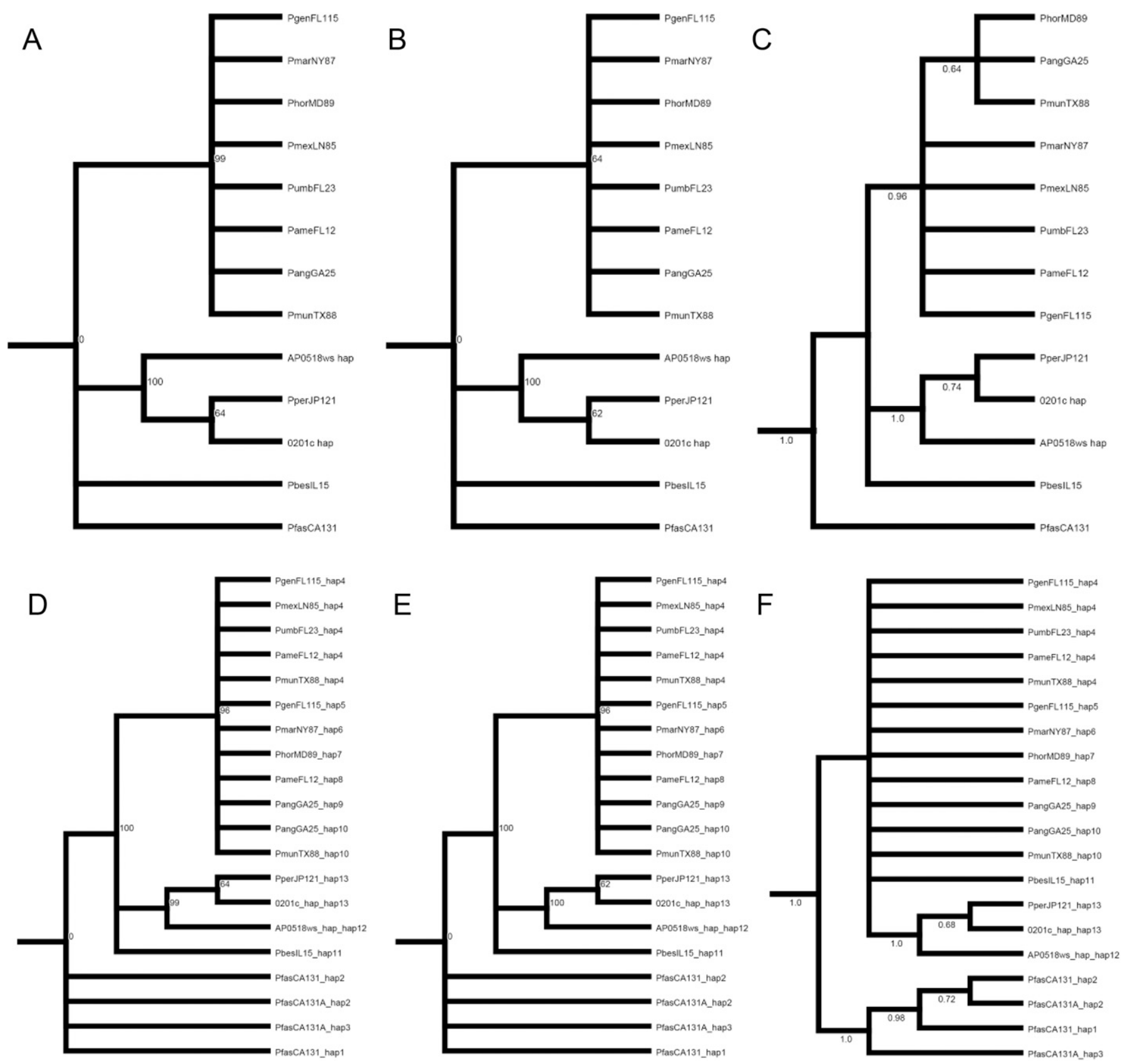

Supplemental Fig. 22. Majority rule consensus trees for MAX3 diploid sequence data using (A) maximum parsimony, (B) maximum parsimony (including gaps), and (C) maximum likelihood; and haplotypic sequence data using (D) maximum parsimony, (E) maximum parsimony (including gaps), and (F) maximum likelihood. Bootstrap values greater than $50 \%$ are described above the branches for maximum parsimony tree. Posterior probability values are described above the branches for maximum likehood tree. Trees are rooted with Prunus fasciculata (PfasCA131). Other species in the cladogram are Prunus americana (PameFL12), Prunus angustifolia (PangGA25), Prunus geniculata (PgenFL115), Prunus hortulana (PhorMD89), Prunus maritima (PmarNY87), Prunus mexicana (PmexLN85), Prunus munsoniana (PmunTX88), Prunus persica (PperJP121, 0201c_hap, AP0518ws_hap), Prunus pumila (PbesIL15), and Prunus umbellata (PumbFL23). 

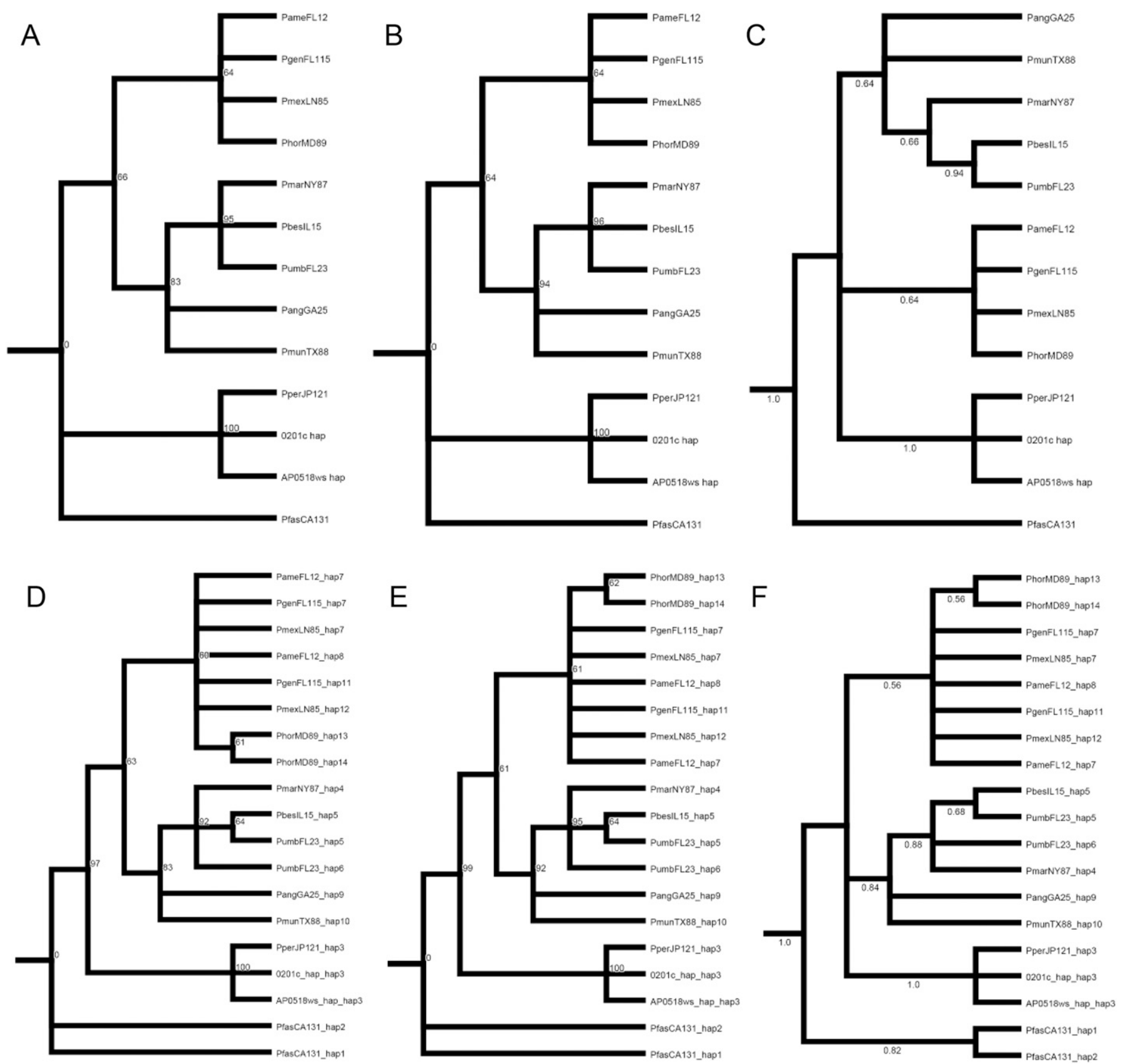

Supplemental Fig. 23. Majority rule consensus trees for MAX4 diploid sequence data using (A) maximum parsimony, (B) maximum parsimony (including gaps), and (C) maximum likelihood; and haplotypic sequence data using (D) maximum parsimony, (E) maximum parsimony (including gaps), and (F) maximum likelihood. Bootstrap values greater than $50 \%$ are described above the branches for maximum parsimony tree. Posterior probability values are described above the branches for maximum likehood tree. Trees are rooted with Prunus fasciculata (PfasCA131). Other species in the cladogram are Prunus americana (PameFL12), Prunus angustifolia (PangGA25), Prunus geniculata (PgenFL115), Prunus hortulana (PhorMD89), Prunus maritima (PmarNY87), Prunus mexicana (PmexLN85), Prunus munsoniana (PmunTX88), Prunus persica (PperJP121, 0201c_hap, AP0518ws_hap), Prunus pumila (PbesIL15), and Prunus umbellata (PumbFL23). 

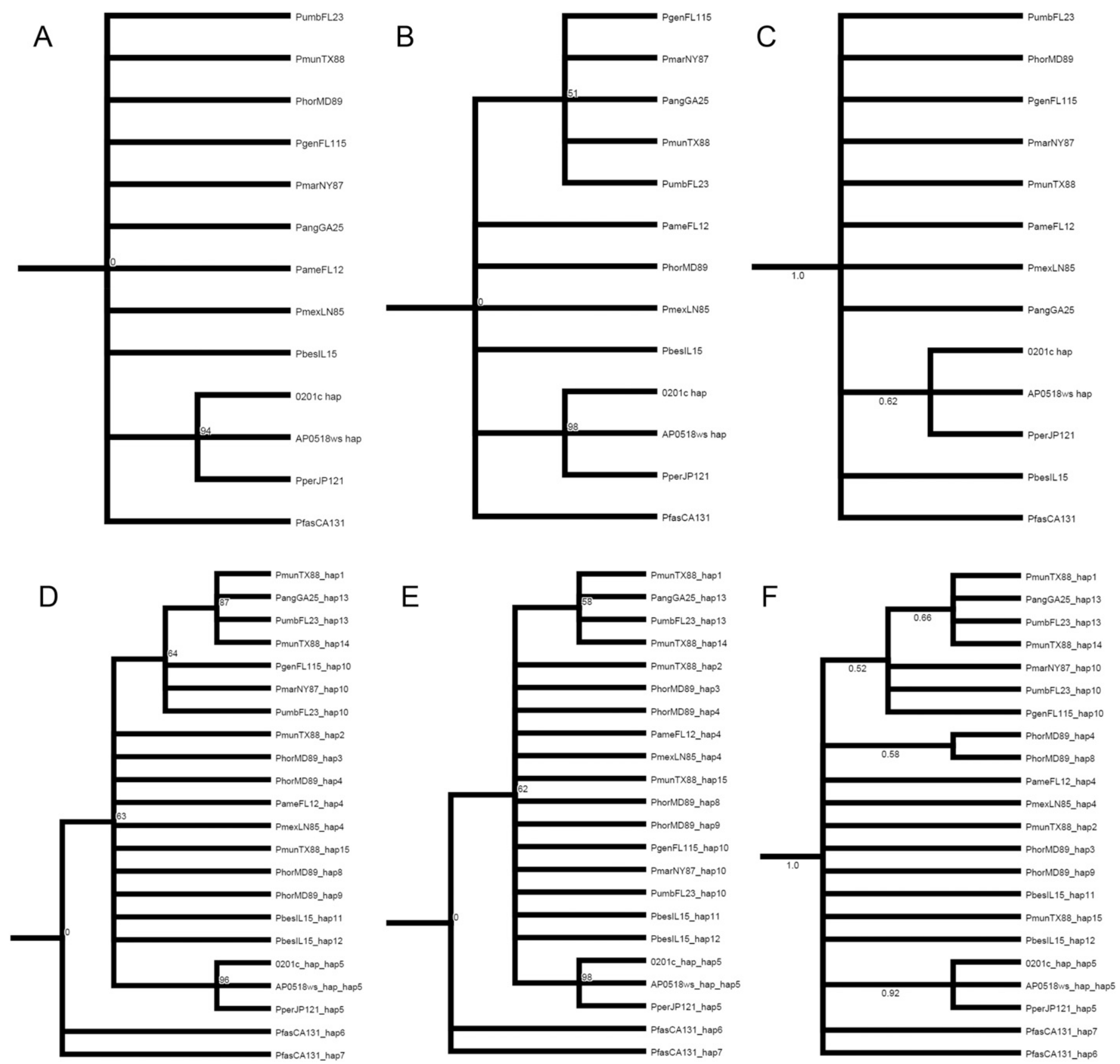

Supplemental Fig. 24. Majority rule consensus trees for PIN diploid sequence data using (A) maximum parsimony, (B) maximum parsimony (including gaps), and (C) maximum likelihood; and haplotypic sequence data using (D) maximum parsimony, (E) maximum parsimony (including gaps), and (F) maximum likelihood. Bootstrap values greater than $50 \%$ are described above the branches for maximum parsimony tree. Posterior probability values are described above the branches for maximum likehood tree. Trees are rooted with Prunus fasciculata (PfasCA131). Other species in the cladogram are Prunus americana (PameFL12), Prunus angustifolia (PangGA25), Prunus geniculata (PgenFL115), Prunus hortulana (PhorMD89), Prunus maritima (PmarNY87), Prunus mexicana (PmexLN85), Prunus munsoniana (PmunTX88), Prunus persica (PperJP121, 0201c_hap, AP0518ws_hap), Prunus pumila (PbesIL15), and Prunus umbellata (PumbFL23). 

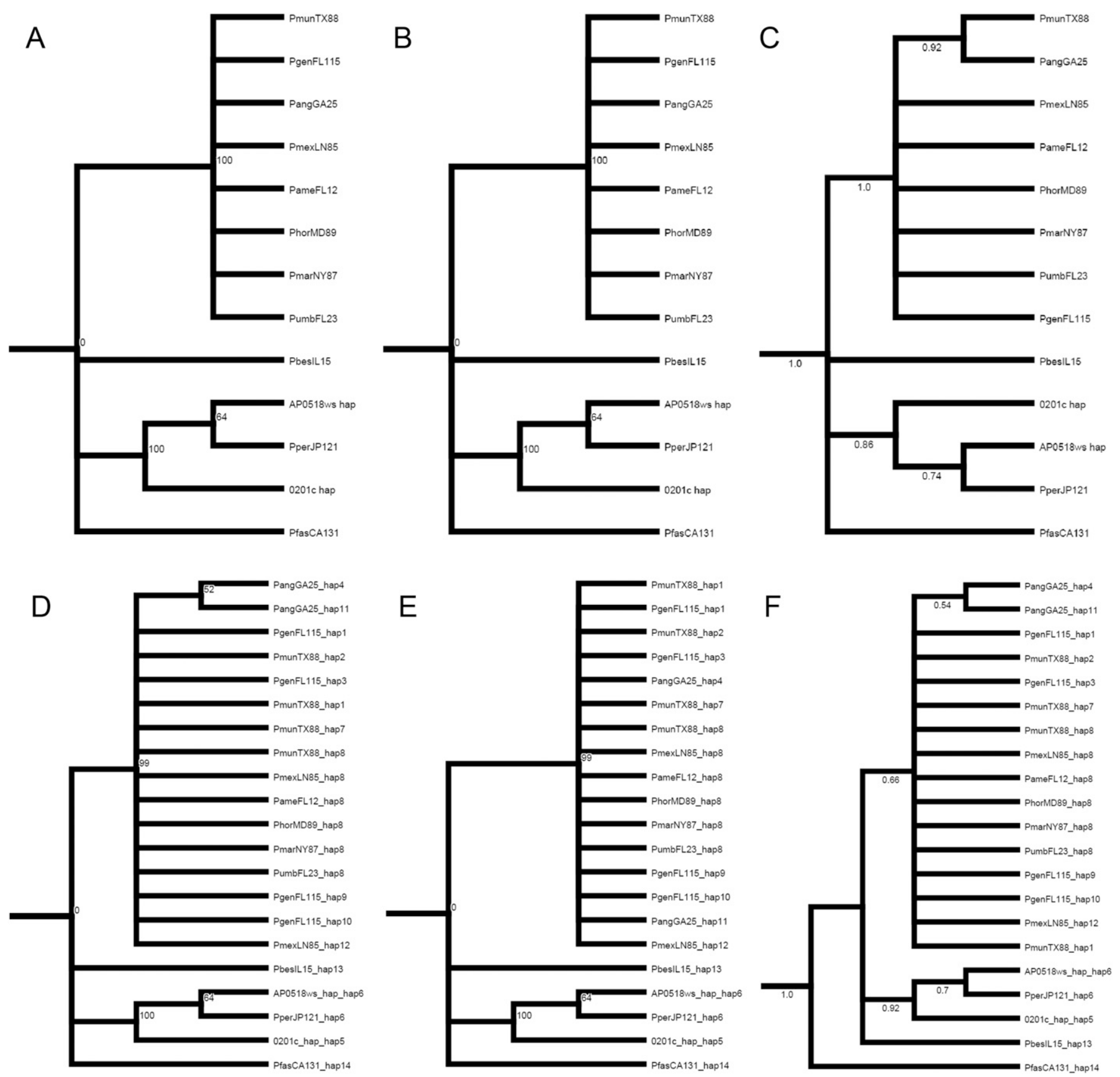

Supplemental Fig. 25. Majority rule consensus trees for RAX1 diploid sequence data using (A) maximum parsimony, (B) maximum parsimony (including gaps), and (C) maximum likelihood; and haplotypic sequence data using (D) maximum parsimony, (E) maximum parsimony (including gaps), and (F) maximum likelihood. Bootstrap values greater than $50 \%$ are described above the branches for maximum parsimony tree. Posterior probability values are described above the branches for maximum likehood tree. Trees are rooted with Prunus fasciculata (PfasCA131). Other species in the cladogram are Prunus americana (PameFL12), Prunus angustifolia (PangGA25), Prunus geniculata (PgenFL115), Prunus hortulana (PhorMD89), Prunus maritima (PmarNY87), Prunus mexicana (PmexLN85), Prunus munsoniana (PmunTX88), Prunus persica (PperJP121, 0201c_hap, AP0518ws_hap), Prunus pumila (PbesIL15), and Prunus umbellata (PumbFL23). 

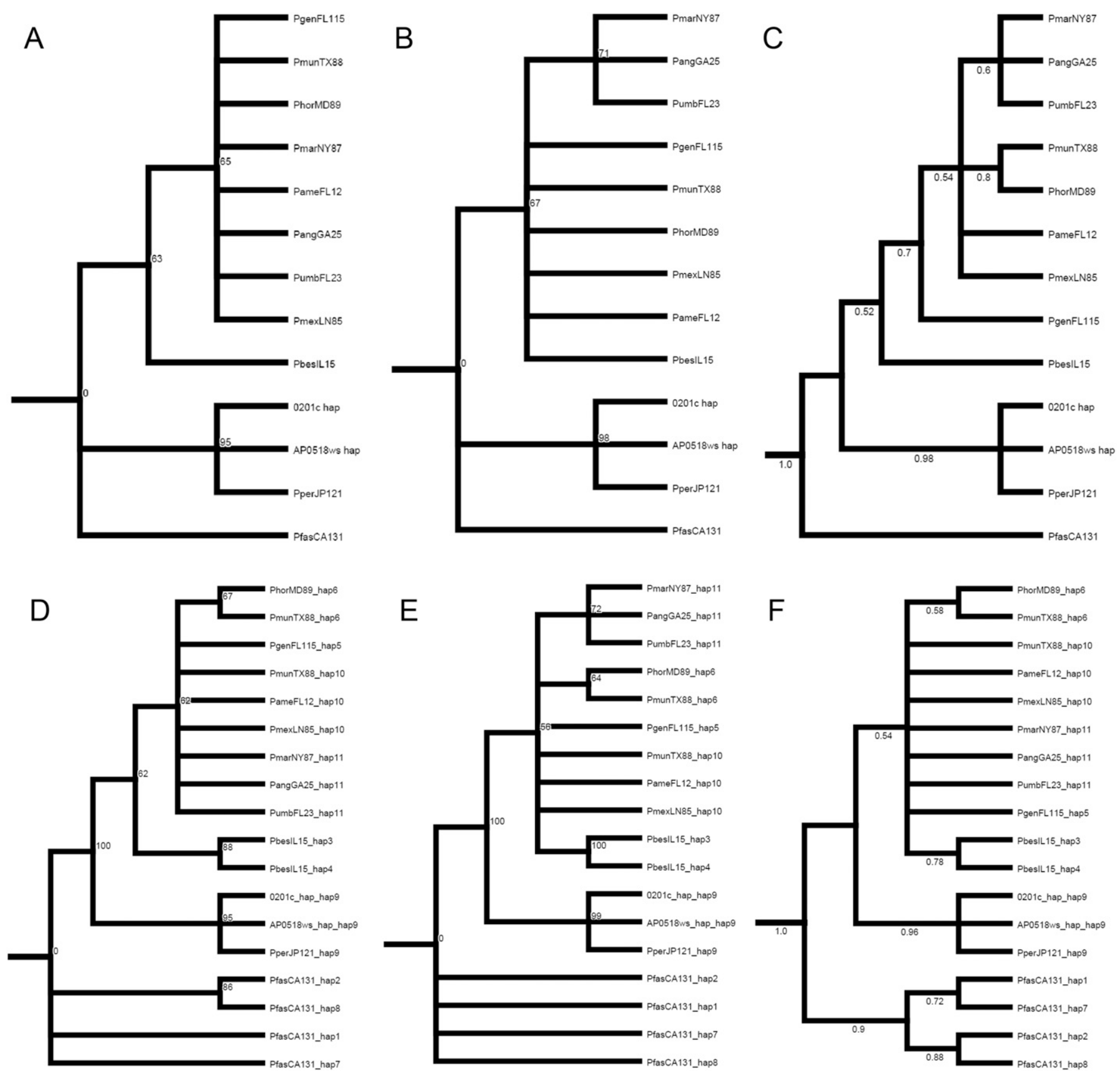

Supplemental Fig. 26. Majority rule consensus trees for RAX2-RAX3 diploid sequence data using (A) maximum parsimony, (B) maximum parsimony (including gaps), and (C) maximum likelihood; and haplotypic sequence data using (D) maximum parsimony, (E) maximum parsimony (including gaps), and (F) maximum likelihood. Bootstrap values greater than $50 \%$ are described above the branches for maximum parsimony tree. Posterior probability values are described above the branches for maximum likehood tree. Trees are rooted with Prunus fasciculata (PfasCA131). Other species in the cladogram are Prunus americana (PameFL12), Prunus angustifolia (PangGA25), Prunus geniculata (PgenFL115), Prunus hortulana (PhorMD89), Prunus maritima (PmarNY87), Prunus mexicana (PmexLN85), Prunus munsoniana (PmunTX88), Prunus persica (PperJP121, 0201c_hap, AP0518ws_hap), Prunus pumila (PbesIL15), and Prunus umbellata (PumbFL23). 

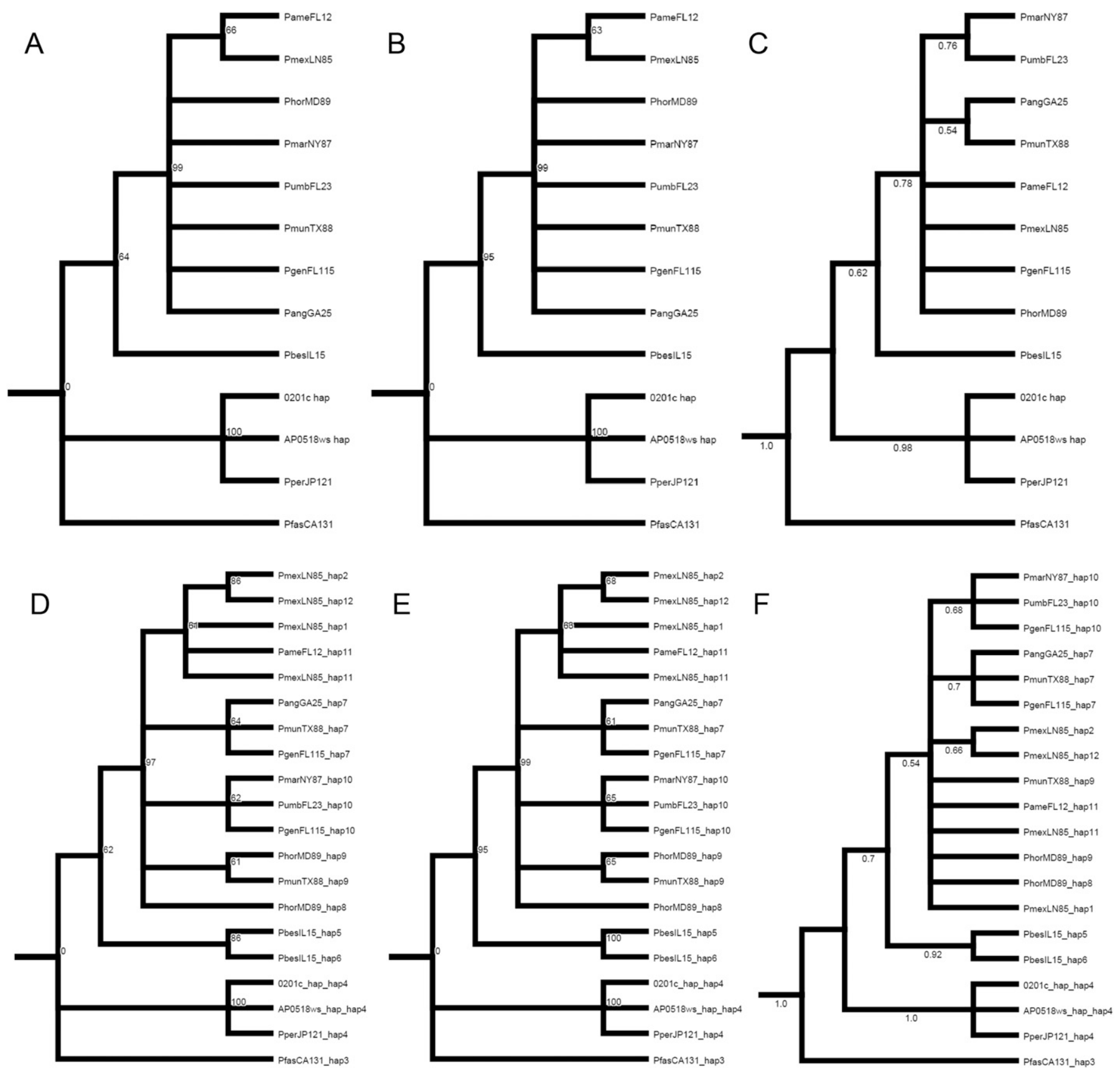

Supplemental Fig. 27. Majority rule consensus trees for REV diploid sequence data using (A) maximum parsimony, (B) maximum parsimony (including gaps), and (C) maximum likelihood; and haplotypic sequence data using (D) maximum parsimony, (E) maximum parsimony (including gaps), and (F) maximum likelihood. Bootstrap values greater than $50 \%$ are described above the branches for maximum parsimony tree. Posterior probability values are described above the branches for maximum likehood tree. Trees are rooted with Prunus fasciculata (PfasCA131). Other species in the cladogram are Prunus americana (PameFL12), Prunus angustifolia (PangGA25), Prunus geniculata (PgenFL115), Prunus hortulana (PhorMD89), Prunus maritima (PmarNY87), Prunus mexicana (PmexLN85), Prunus munsoniana (PmunTX88), Prunus persica (PperJP121, 0201c_hap, AP0518ws_hap), Prunus pumila (PbesIL15), and Prunus umbellata (PumbFL23). 

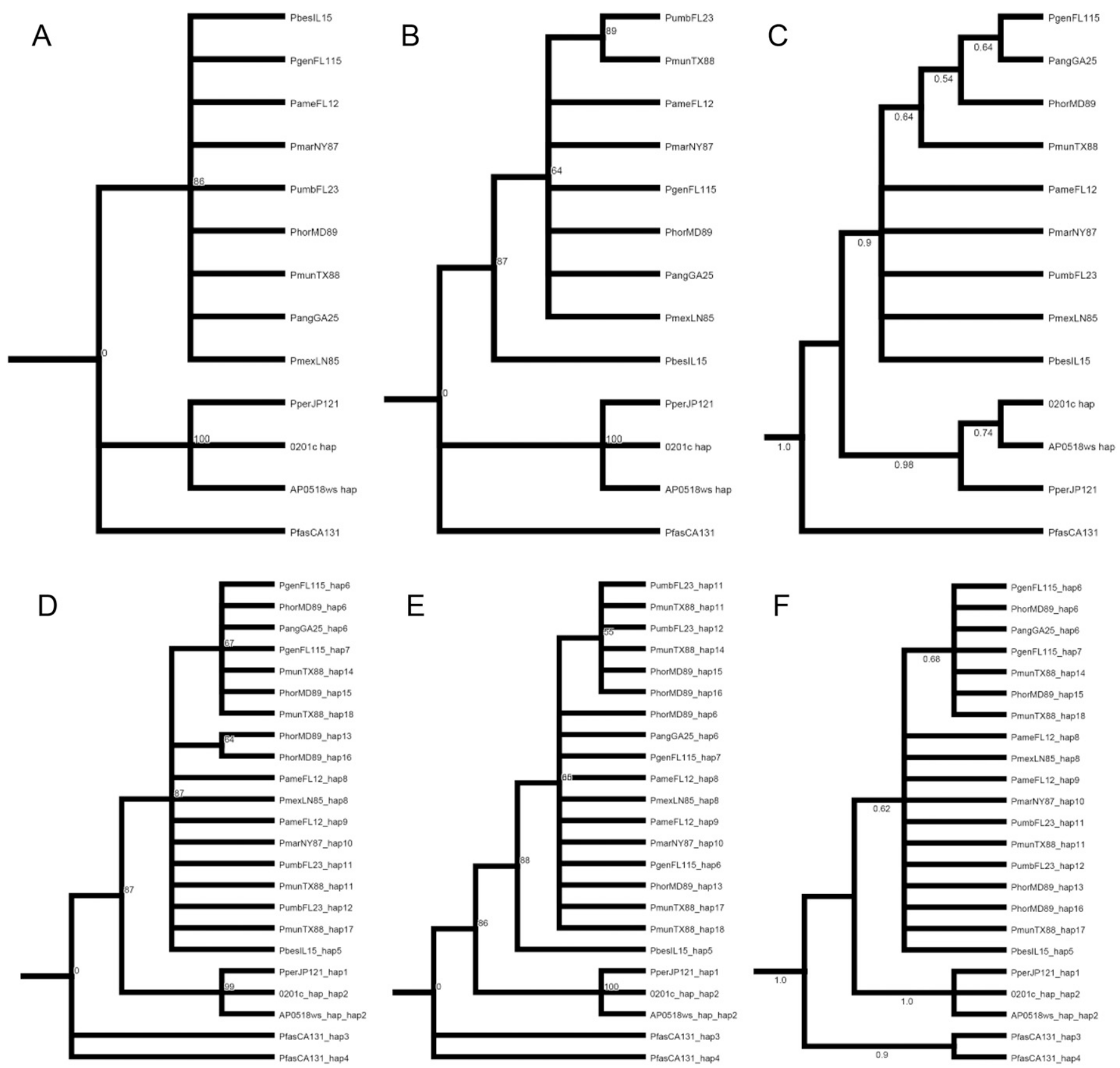

Supplemental Fig. 28. Majority rule consensus trees for SPS diploid sequence data using (A) maximum parsimony, (B) maximum parsimony (including gaps), and (C) maximum likelihood; and haplotypic sequence data using (D) maximum parsimony, (E) maximum parsimony (including gaps), and (F) maximum likelihood. Bootstrap values greater than $50 \%$ are described above the branches for maximum parsimony tree. Posterior probability values are described above the branches for maximum likehood tree. Trees are rooted with Prunus fasciculata (PfasCA131). Other species in the cladogram are Prunus americana (PameFL12), Prunus angustifolia (PangGA25), Prunus geniculata (PgenFL115), Prunus hortulana (PhorMD89), Prunus maritima (PmarNY87), Prunus mexicana (PmexLN85), Prunus munsoniana (PmunTX88), Prunus persica (PperJP121, 0201c_hap, AP0518ws_hap), Prunus pumila (PbesIL15), and Prunus umbellata (PumbFL23). 

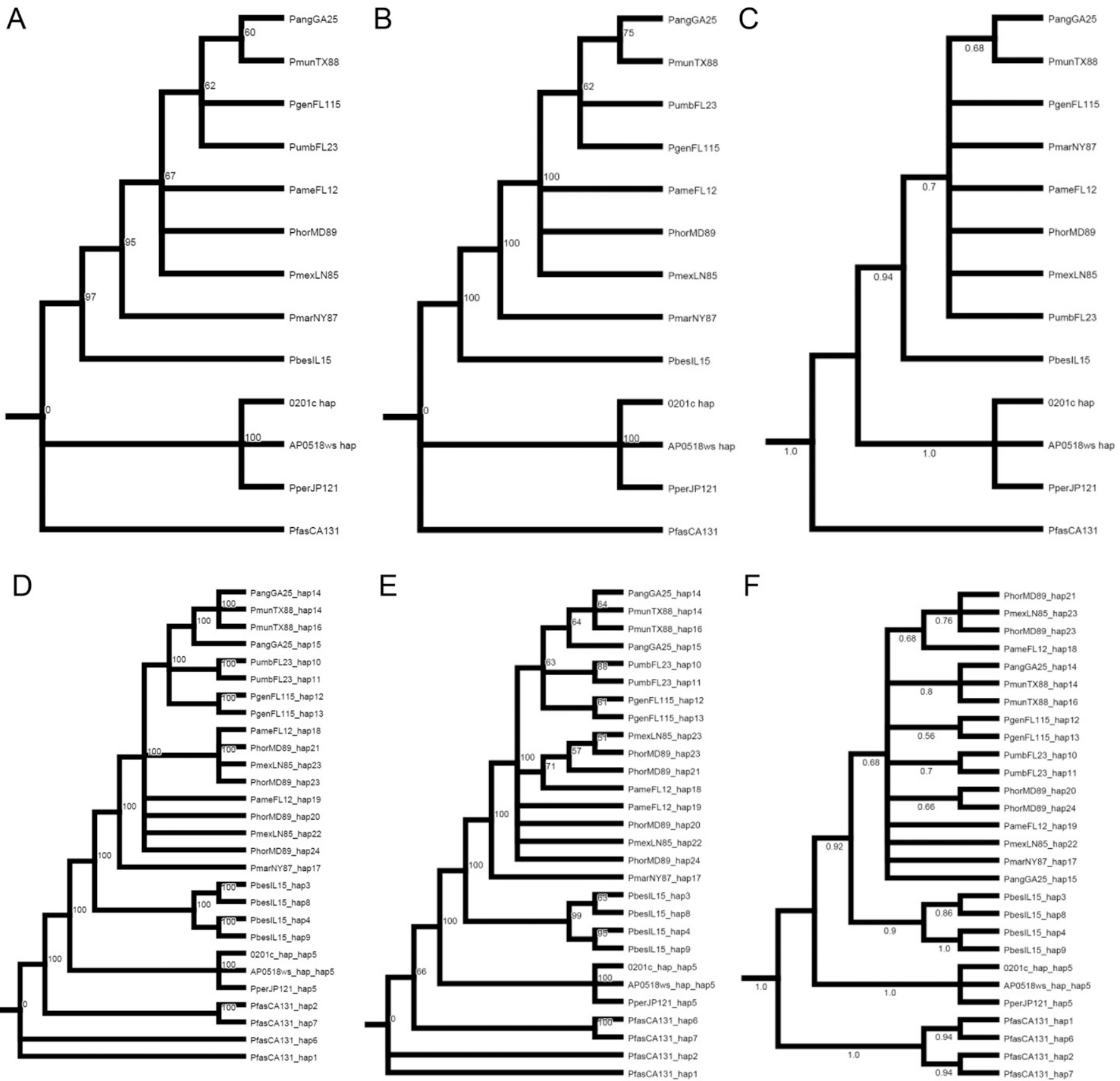

Supplemental Fig. 29. Majority rule consensus trees for AGL24 diploid sequence data using (A) maximum parsimony, (B) maximum parsimony (including gaps), and $(\mathbf{C})$ maximum likelihood; and haplotypic sequence data using $(\mathbf{D})$ maximum parsimony, (E) maximum parsimony (including gaps), and (F) maximum likelihood. Bootstrap values greater than $50 \%$ are described above the branches for maximum parsimony tree. Posterior probability values are described above the branches for maximum likehood tree. Trees are rooted with Prunus fasciculata (PfasCA131). Other species in the cladogram are Prunus americana (PameFL12), Prunus angustifolia (PangGA25), Prunus geniculata (PgenFL115), Prunus hortulana (PhorMD89), Prunus maritima (PmarNY87), Prunus mexicana (PmexLN85), Prunus munsoniana (PmunTX88), Prunus persica (PperJP121, 0201c_hap, AP0518ws_hap), Prunus pumila (PbesIL15), and Prunus umbellata (PumbFL23). 

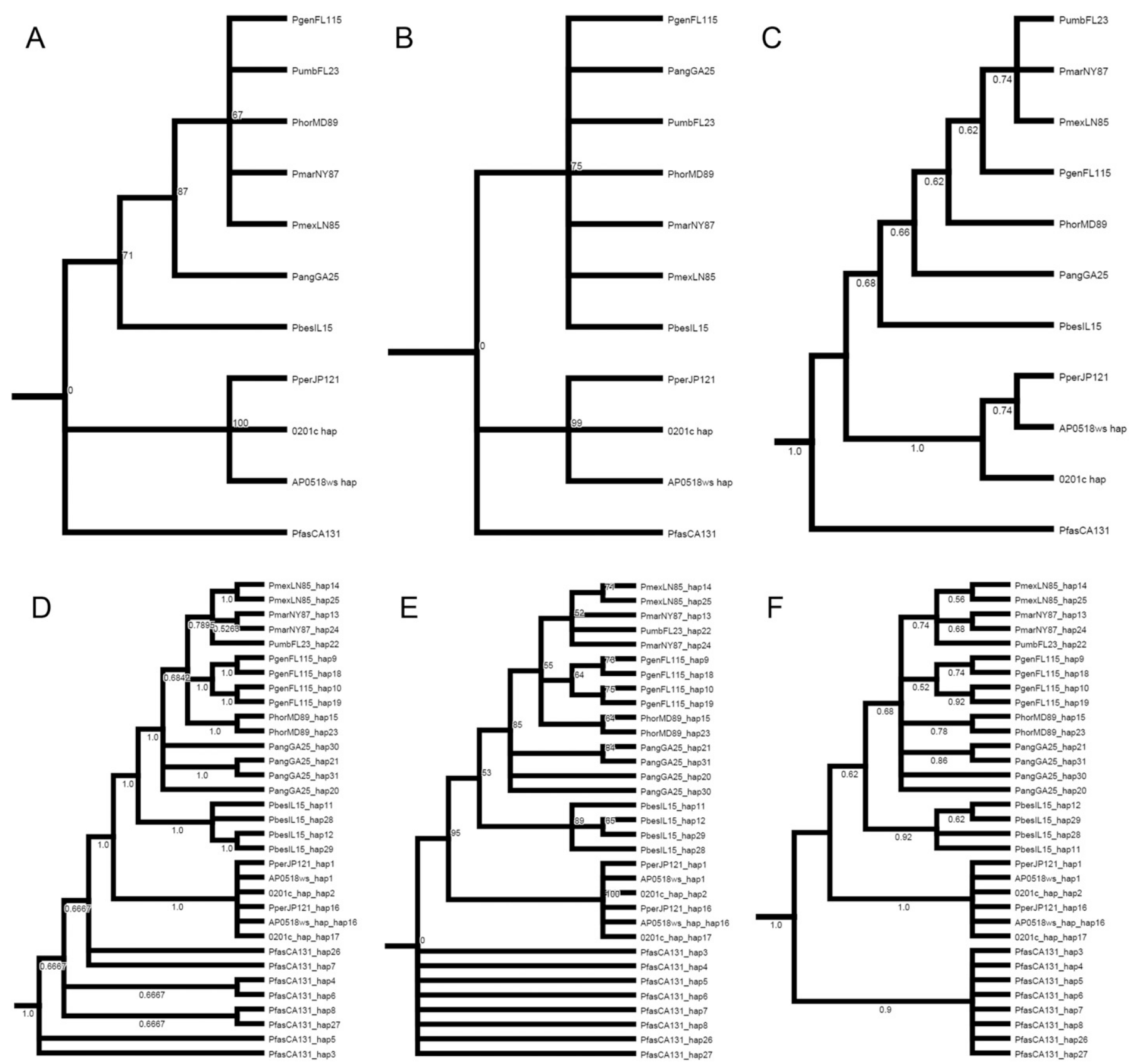

Supplemental Fig. 30. Majority rule consensus trees for AGL20-SOC1 diploid sequence data using (A) maximum parsimony, (B) maximum parsimony (including gaps), and (C) maximum likelihood; and haplotypic sequence data using (D) maximum parsimony, (E) maximum parsimony (including gaps), and (F) maximum likelihood. Bootstrap values greater than $50 \%$ are described above the branches for maximum parsimony tree. Posterior probability values are described above the branches for maximum likehood tree. Trees are rooted with Prunus fasciculata (PfasCA131). Other species in the cladogram are Prunus americana (PameFL12), Prunus angustifolia (PangGA25), Prunus geniculata (PgenFL115), Prunus hortulana (PhorMD89), Prunus maritima (PmarNY87), Prunus mexicana (PmexLN85), Prunus munsoniana (PmunTX88), Prunus persica (PperJP121, 0201c_hap, AP0518ws_hap), Prunus pumila (PbesIL15), and Prunus umbellata (PumbFL23). 

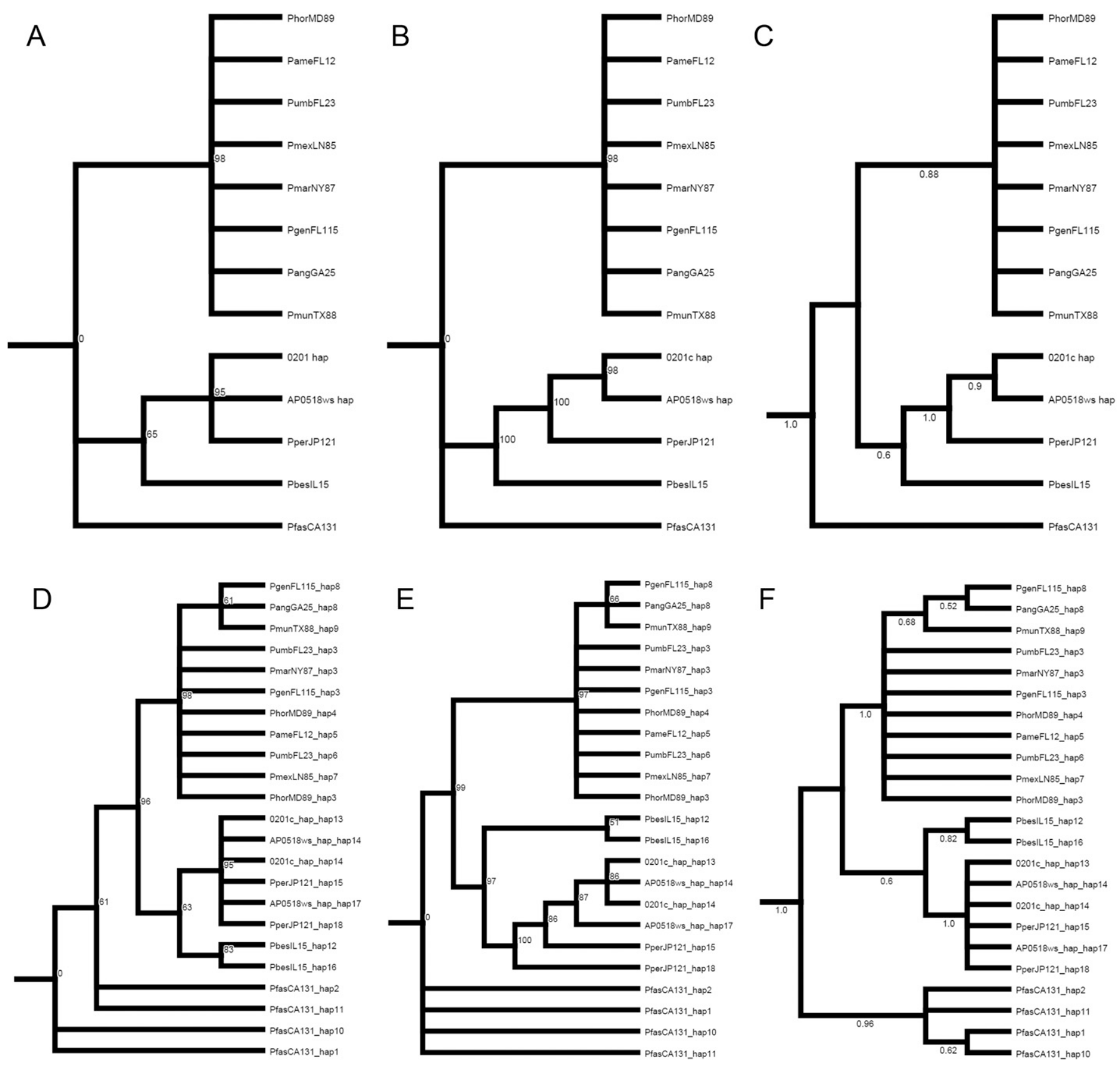

Supplemental Fig. 31. Majority rule consensus trees for BFT diploid sequence data using (A) maximum parsimony, (B) maximum parsimony (including gaps), and (C) maximum likelihood; and haplotypic sequence data using (D) maximum parsimony, (E) maximum parsimony (including gaps), and (F) maximum likelihood. Bootstrap values greater than $50 \%$ are described above the branches for maximum parsimony tree. Posterior probability values are described above the branches for maximum likehood tree. Trees are rooted with Prunus fasciculata (PfasCA131). Other species in the cladogram are Prunus americana (PameFL12), Prunus angustifolia (PangGA25), Prunus geniculata (PgenFL115), Prunus hortulana (PhorMD89), Prunus maritima (PmarNY87), Prunus mexicana (PmexLN85), Prunus munsoniana (PmunTX88), Prunus persica (PperJP121, 0201c_hap, AP0518ws_hap), Prunus pumila (PbesIL15), and Prunus umbellata (PumbFL23). 

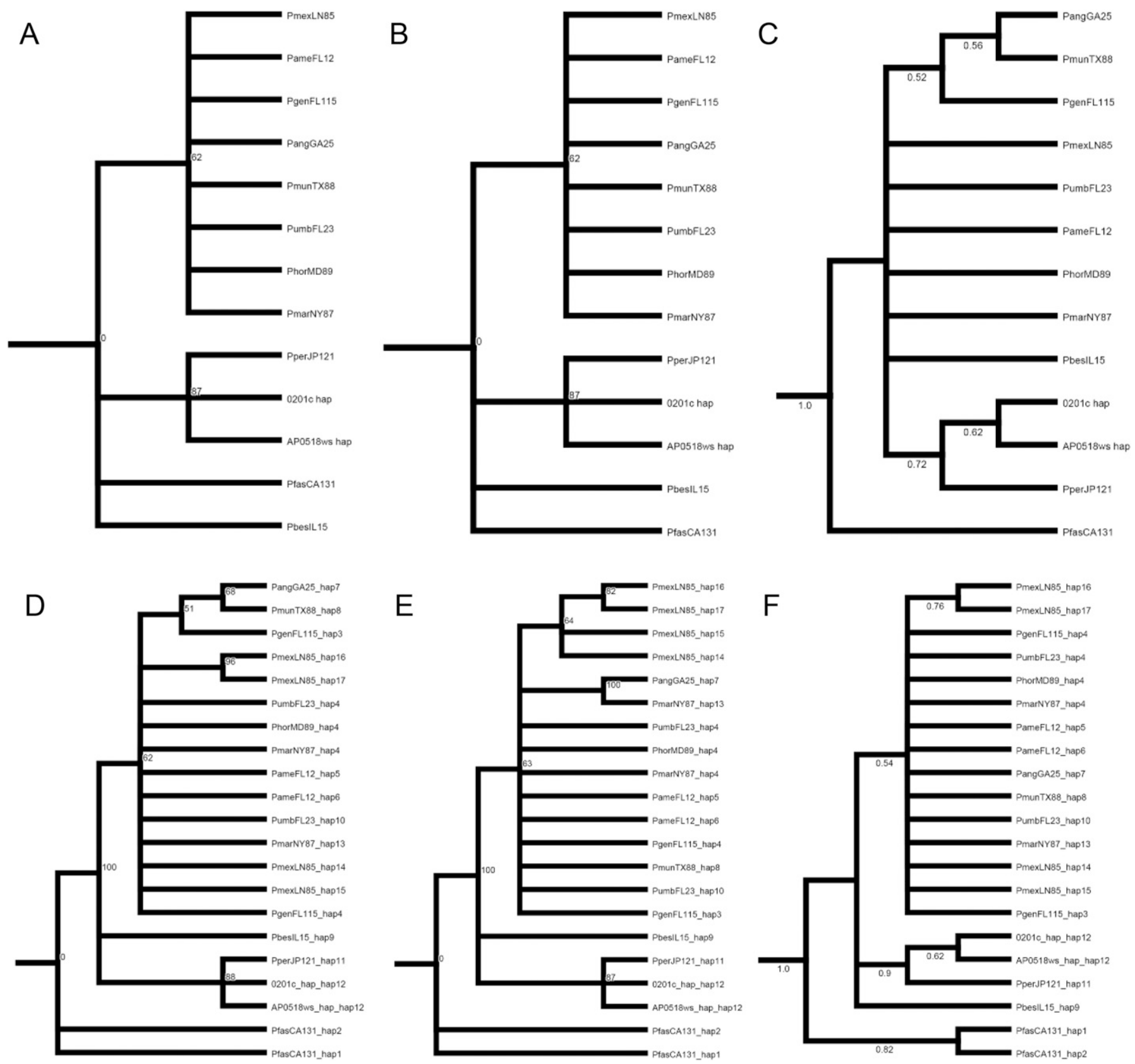

Supplemental Fig. 32. Majority rule consensus trees for BRM diploid sequence data using (A) maximum parsimony, (B) maximum parsimony (including gaps), and (C) maximum likelihood; and haplotypic sequence data using (D) maximum parsimony, (E) maximum parsimony (including gaps), and (F) maximum likelihood. Bootstrap values greater than $50 \%$ are described above the branches for maximum parsimony tree. Posterior probability values are described above the branches for maximum likehood tree. Trees are rooted with Prunus fasciculata (PfasCA131). Other species in the cladogram are Prunus americana (PameFL12), Prunus angustifolia (PangGA25), Prunus geniculata (PgenFL115), Prunus hortulana (PhorMD89), Prunus maritima (PmarNY87), Prunus mexicana (PmexLN85), Prunus munsoniana (PmunTX88), Prunus persica (PperJP121, 0201c_hap, AP0518ws_hap), Prunus pumila (PbesIL15), and Prunus umbellata (PumbFL23). 

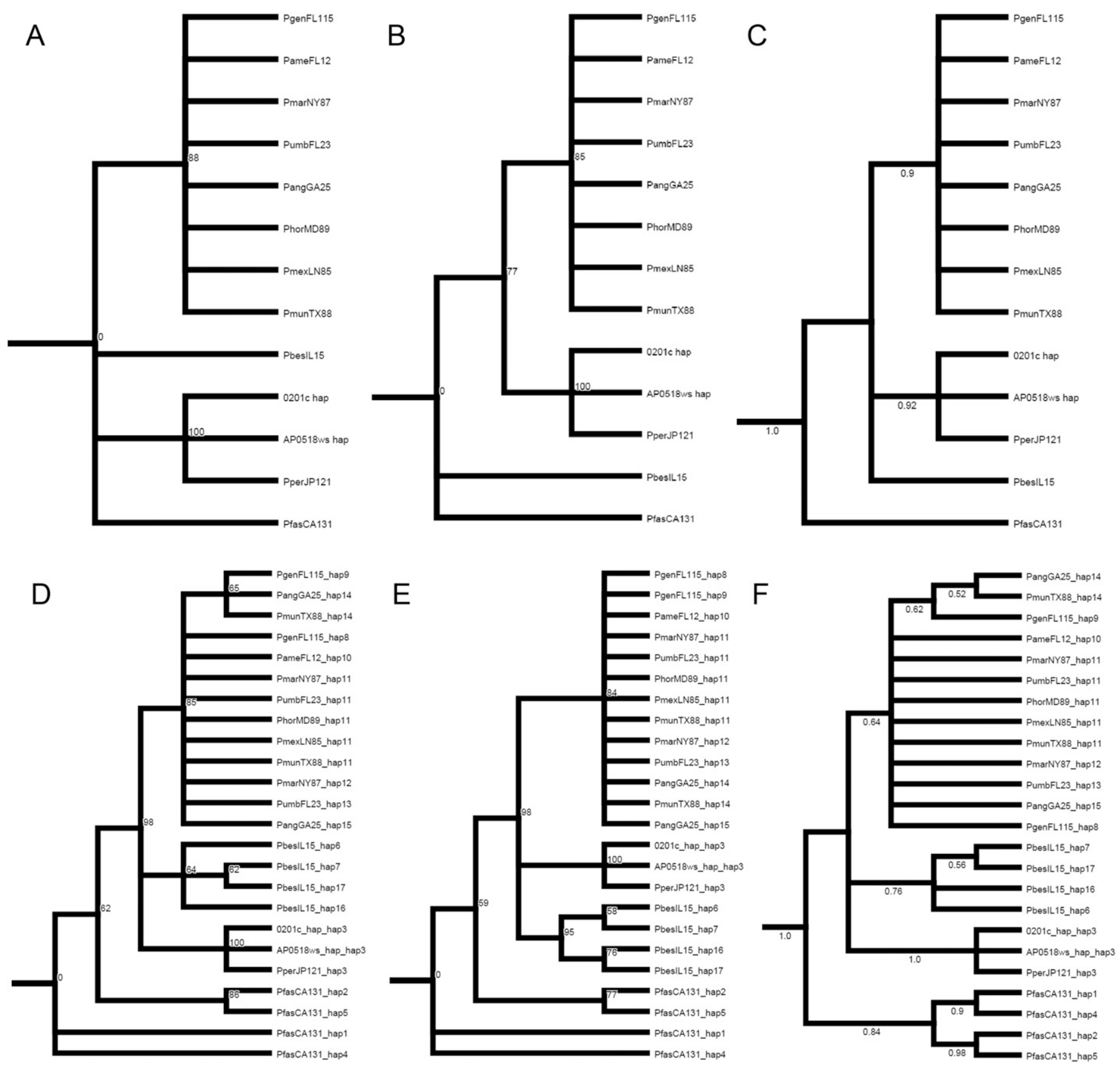

Supplemental Fig. 33. Majority rule consensus trees for CO diploid sequence data using (A) maximum parsimony, (B) maximum parsimony (including gaps), and (C) maximum likelihood; and haplotypic sequence data using (D) maximum parsimony, (E) maximum parsimony (including gaps), and (F) maximum likelihood. Bootstrap values greater than $50 \%$ are described above the branches for maximum parsimony tree. Posterior probability values are described above the branches for maximum likehood tree. Trees are rooted with Prunus fasciculata (PfasCA131). Other species in the cladogram are Prunus americana (PameFL12), Prunus angustifolia (PangGA25), Prunus geniculata (PgenFL115), Prunus hortulana (PhorMD89), Prunus maritima (PmarNY87), Prunus mexicana (PmexLN85), Prunus munsoniana (PmunTX88), Prunus persica (PperJP121, 0201c_hap, AP0518ws_hap), Prunus pumila (PbesIL15), and Prunus umbellata (PumbFL23). 

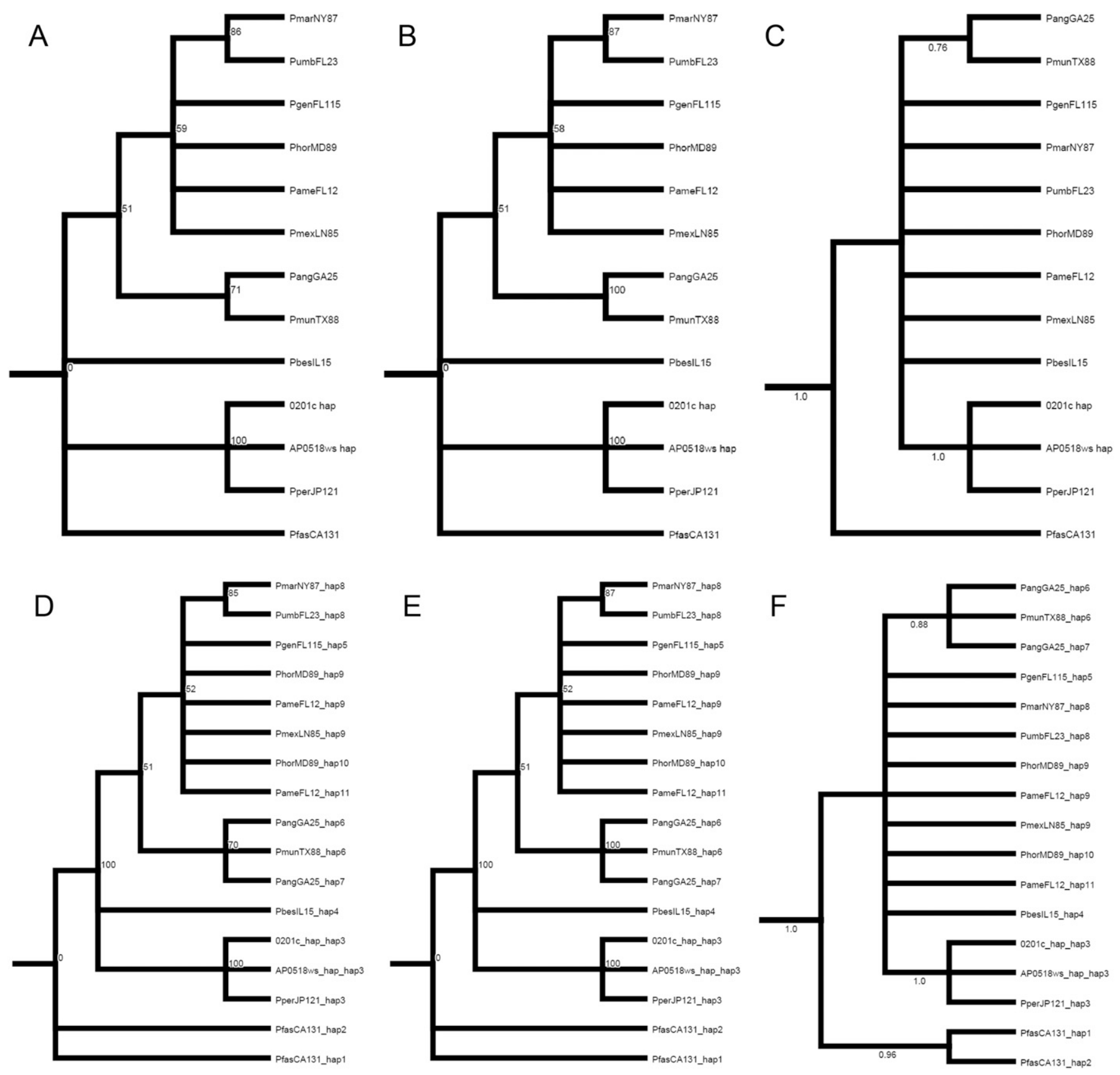

Supplemental Fig. 34. Majority rule consensus trees for CRY1 diploid sequence data using (A) maximum parsimony, (B) maximum parsimony (including gaps), and $(\mathbf{C})$ maximum likelihood; and haplotypic sequence data using (D) maximum parsimony, (E) maximum parsimony (including gaps), and (F) maximum likelihood. Bootstrap values greater than $50 \%$ are described above the branches for maximum parsimony tree. Posterior probability values are described above the branches for maximum likehood tree. Trees are rooted with Prunus fasciculata (PfasCA131). Other species in the cladogram are Prunus americana (PameFL12), Prunus angustifolia (PangGA25), Prunus geniculata (PgenFL115), Prunus hortulana (PhorMD89), Prunus maritima (PmarNY87), Prunus mexicana (PmexLN85), Prunus munsoniana (PmunTX88), Prunus persica (PperJP121, 0201c_hap, AP0518ws_hap), Prunus pumila (PbesIL15), and Prunus umbellata (PumbFL23). 

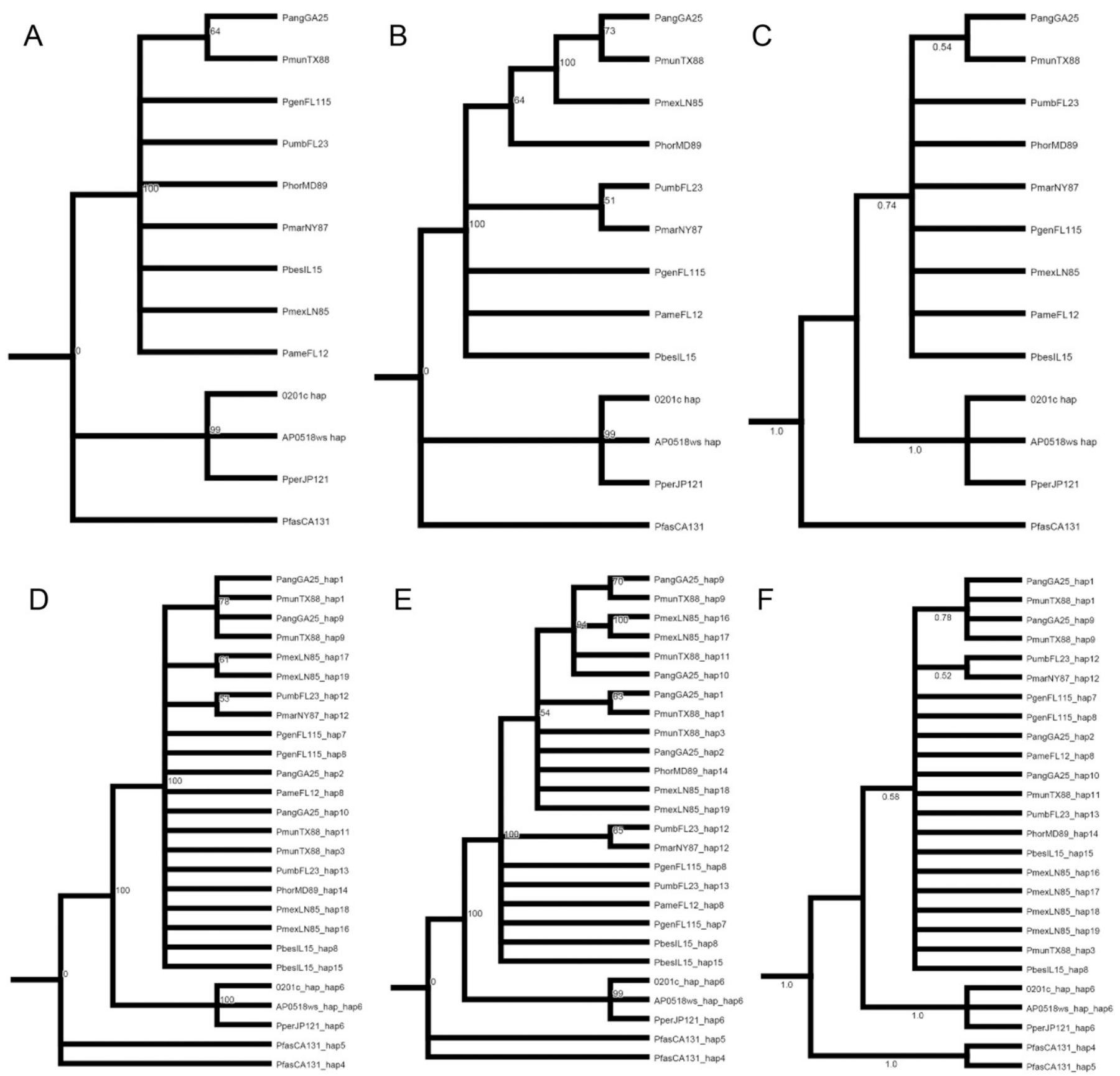

Supplemental Fig. 35. Majority rule consensus trees for CRY2 diploid sequence data using (A) maximum parsimony, (B) maximum parsimony (including gaps), and (C) maximum likelihood; and haplotypic sequence data using (D) maximum parsimony, (E) maximum parsimony (including gaps), and (F) maximum likelihood. Bootstrap values greater than $50 \%$ are described above the branches for maximum parsimony tree. Posterior probability values are described above the branches for maximum likehood tree. Trees are rooted with Prunus fasciculata (PfasCA131). Other species in the cladogram are Prunus americana (PameFL12), Prunus angustifolia (PangGA25), Prunus geniculata (PgenFl115), Prunus hortulana (PhorMD89), Prunus maritima (PmarNY87), Prunus mexicana (PmexLN85), Prunus munsoniana (PmunTX88), Prunus persica (PperJP121, 0201c_hap, AP0518ws_hap), Prunus pumila (PbesIL15), and Prunus umbellata (PumbFL23). 

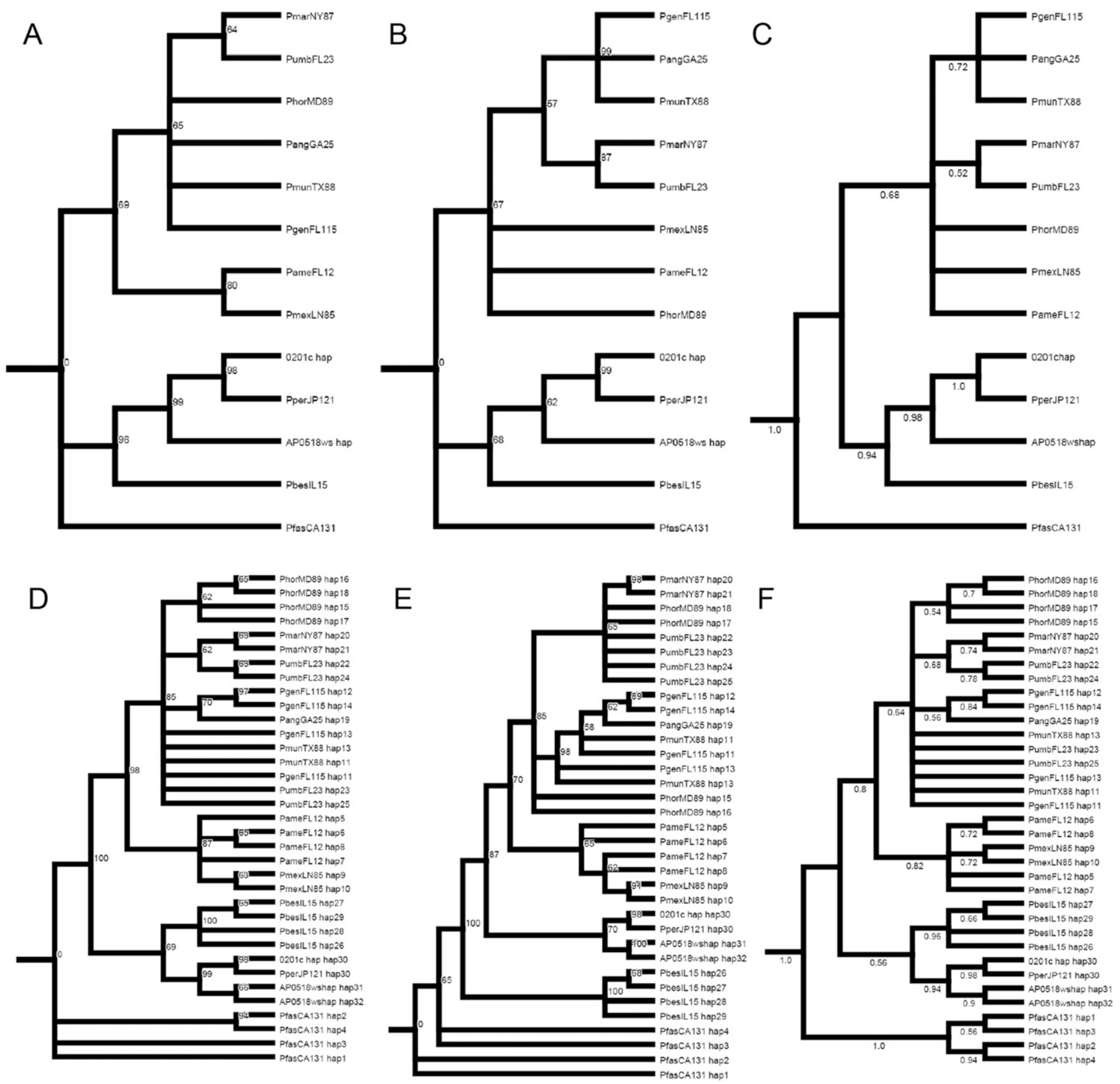

Supplemental Fig. 36. Majority rule consensus trees for ELF6 diploid sequence data using (A) maximum parsimony, (B) maximum parsimony (including gaps), and (C) maximum likelihood; and haplotypic sequence data using (D) maximum parsimony, (E) maximum parsimony (including gaps), and (F) maximum likelihood. Bootstrap values greater than $50 \%$ are described above the branches for maximum parsimony tree. Posterior probability values are described above the branches for maximum likehood tree. Trees are rooted with Prunus fasciculata (PfasCA131). Other species in the cladogram are Prunus americana (PameFL12), Prunus angustifolia (PangGA25), Prunus geniculata (PgenFL115), Prunus hortulana (PhorMD89), Prunus maritima (PmarNY87), Prunus mexicana (PmexLN85), Prunus munsoniana (PmunTX88), Prunus persica (PperJP121, 0201c_hap, AP0518ws_hap), Prunus pumila (PbesIL15), and Prunus umbellata (PumbFL23). 

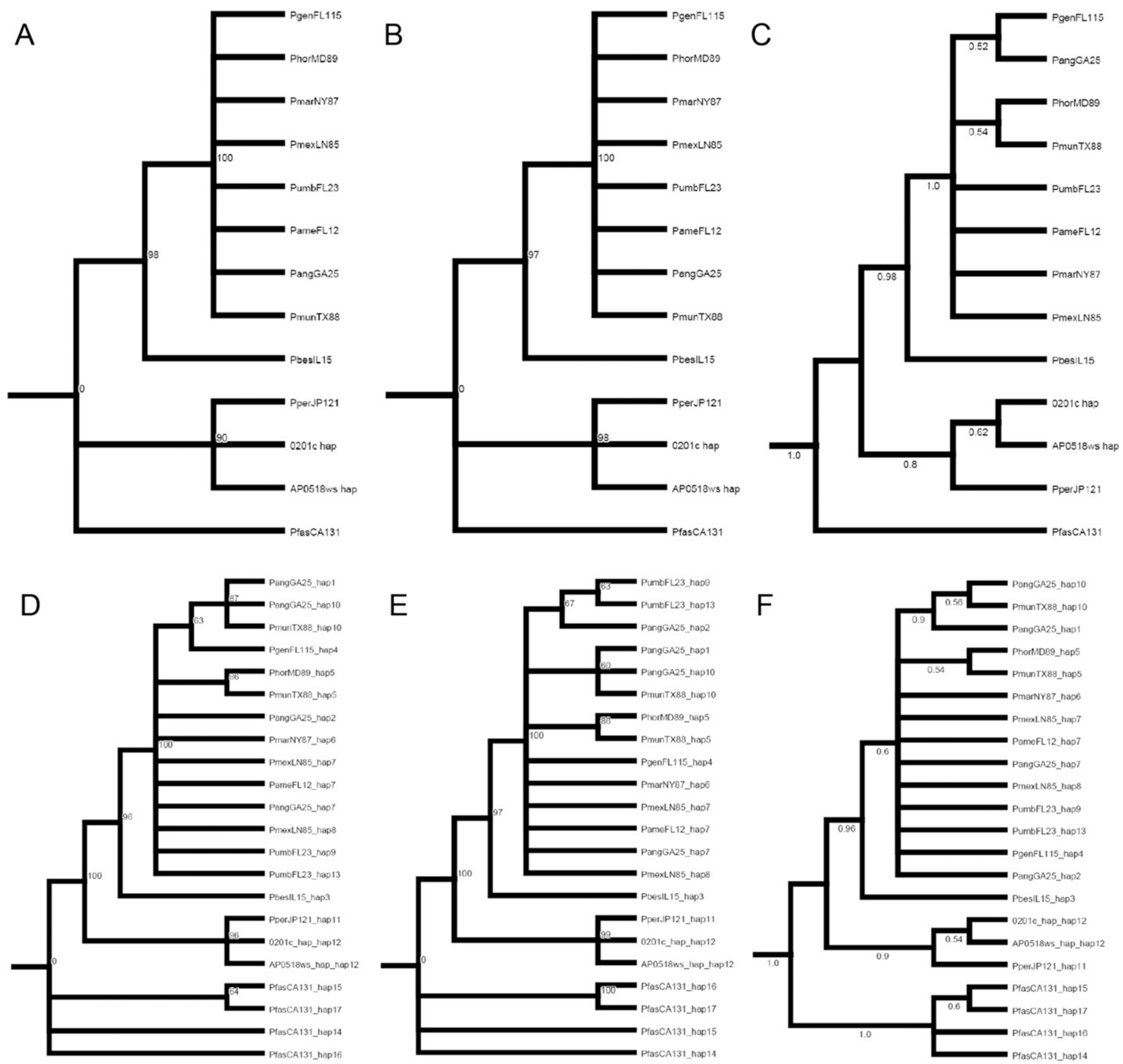

Supplemental Fig. 37. Majority rule consensus trees for FD diploid sequence data using (A) maximum parsimony, (B) maximum parsimony (including gaps), and (C) maximum likelihood; and haplotypic sequence data using (D) maximum parsimony, (E) maximum parsimony (including gaps), and (F) maximum likelihood. Bootstrap values greater than $50 \%$ are described above the branches for maximum parsimony tree. Posterior probability values are described above the branches for maximum likehood tree. Trees are rooted with Prunus fasciculata (PfasCA131). Other species in the cladogram are Prunus americana (PameFL12), Prunus angustifolia (PangGA25), Prunus geniculata (PgenFL115), Prunus hortulana (PhorMD89), Prunus maritima (PmarNY87), Prunus mexicana (PmexLN85), Prunus munsoniana (PmunTX88), Prunus persica (PperJP121, 0201c_hap, AP0518ws_hap), Prunus pumila (PbesIL15), and Prunus umbellata (PumbFL23). 

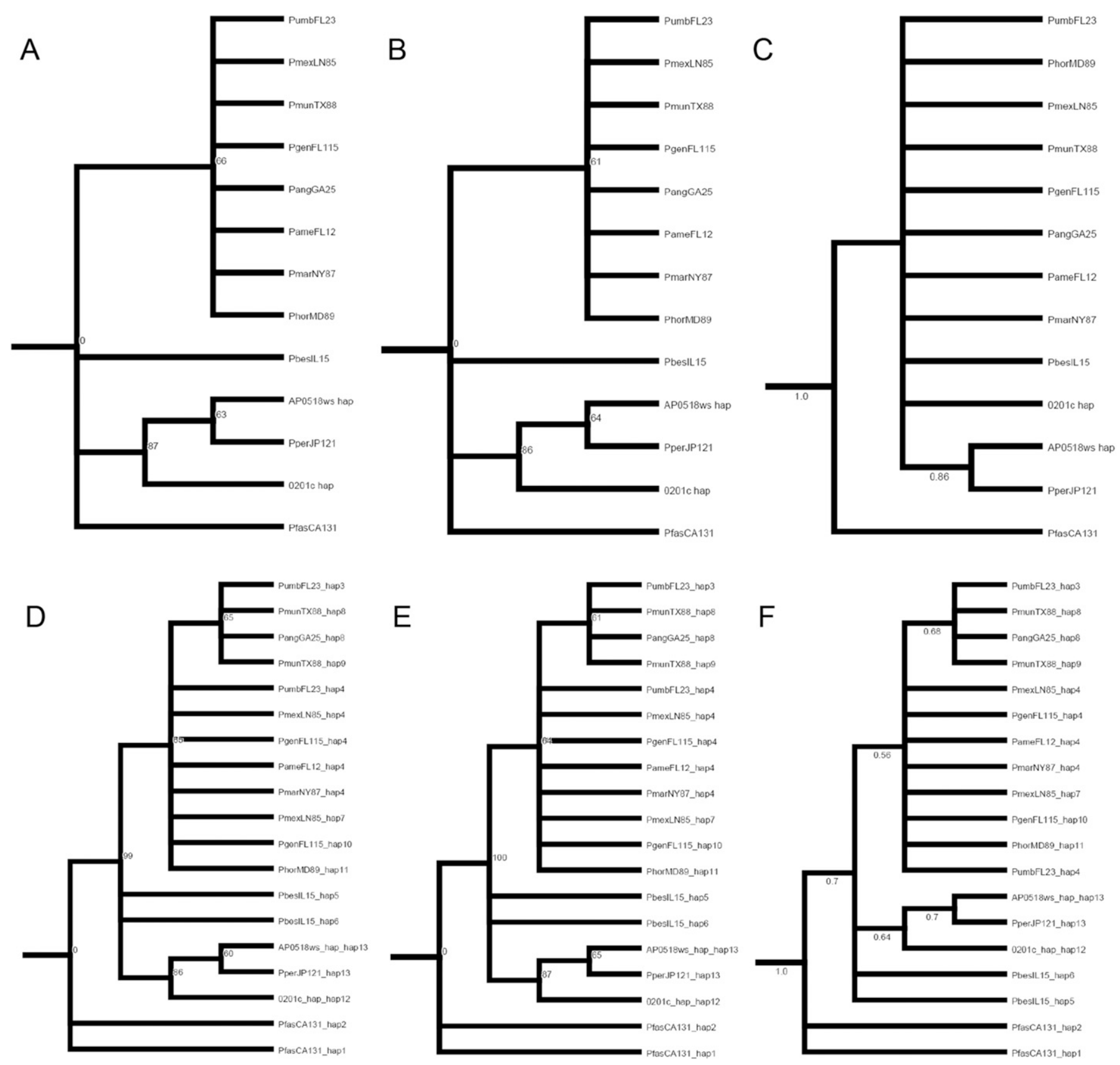

Supplemental Fig. 38. Majority rule consensus trees for FD1 diploid sequence data using (A) maximum parsimony, (B) maximum parsimony (including gaps), and (C) maximum likelihood; and haplotypic sequence data using (D) maximum parsimony, (E) maximum parsimony (including gaps), and (F) maximum likelihood. Bootstrap values greater than $50 \%$ are described above the branches for maximum parsimony tree. Posterior probability values are described above the branches for maximum likehood tree. Trees are rooted with Prunus fasciculata (PfasCA131). Other species in the cladogram are Prunus americana (PameFL12), Prunus angustifolia (PangGA25), Prunus geniculata (PgenFL115), Prunus hortulana (PhorMD89), Prunus maritima (PmarNY87), Prunus mexicana (PmexLN85), Prunus munsoniana (PmunTX88), Prunus persica (PperJP121, 0201c_hap, AP0518ws_hap), Prunus pumila (PbesIL15), and Prunus umbellata (PumbFL23). 

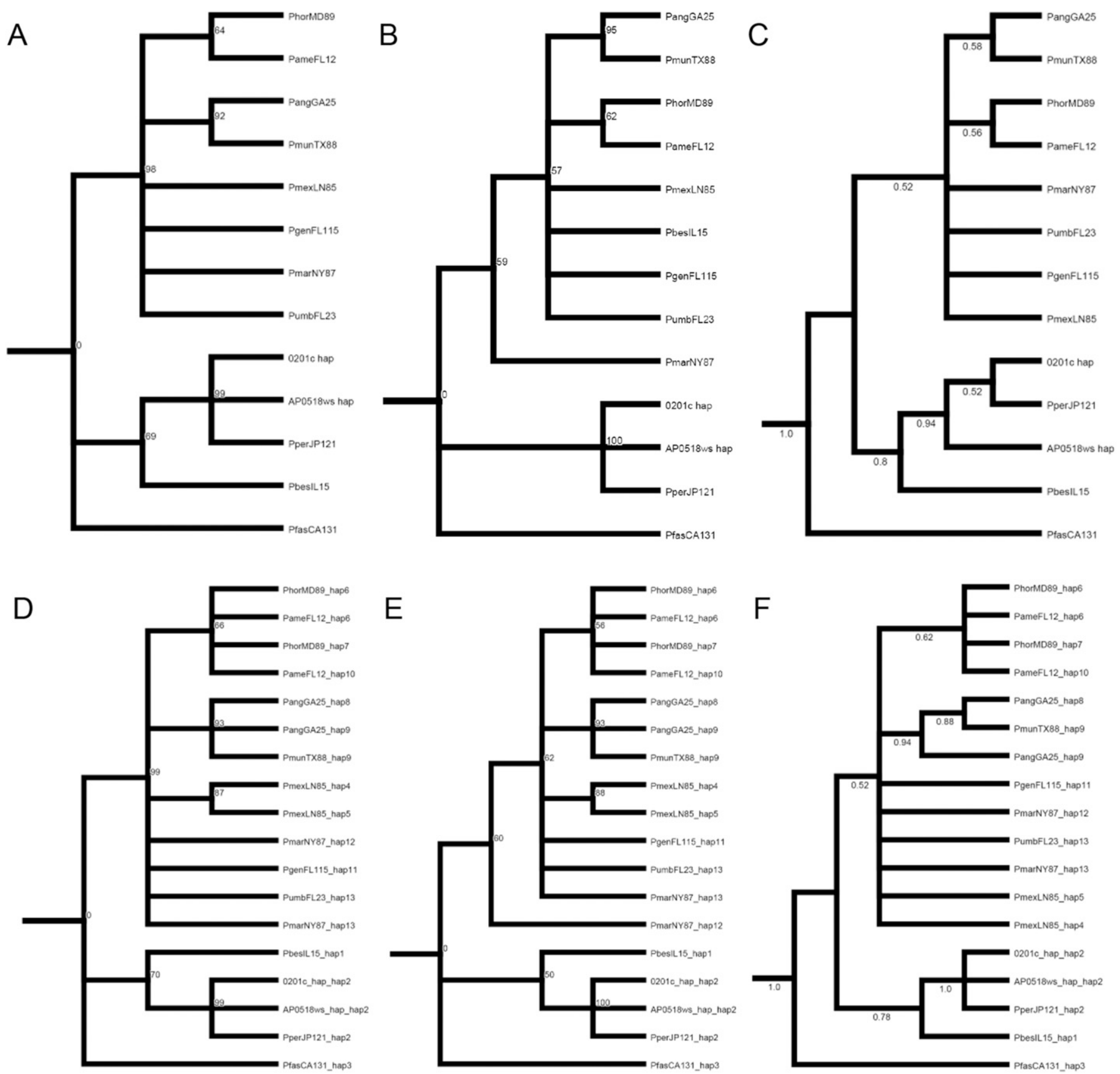

Supplemental Fig. 39. Majority rule consensus trees for FG diploid sequence data using (A) maximum parsimony, (B) maximum parsimony (including gaps), and (C) maximum likelihood; and haplotypic sequence data using (D) maximum parsimony, (E) maximum parsimony (including gaps), and (F) maximum likelihood. Bootstrap values greater than $50 \%$ are described above the branches for maximum parsimony tree. Posterior probability values are described above the branches for maximum likehood tree. Trees are rooted with Prunus fasciculata (PfasCA131). Other species in the cladogram are Prunus americana (PameFL12), Prunus angustifolia (PangGA25), Prunus geniculata (PgenFL115), Prunus hortulana (PhorMD89), Prunus maritima (PmarNY87), Prunus mexicana (PmexLN85), Prunus munsoniana (PmunTX88), Prunus persica (PperJP121, 0201c_hap, AP0518ws_hap), Prunus pumila (PbesIL15), and Prunus umbellata (PumbFL23). 

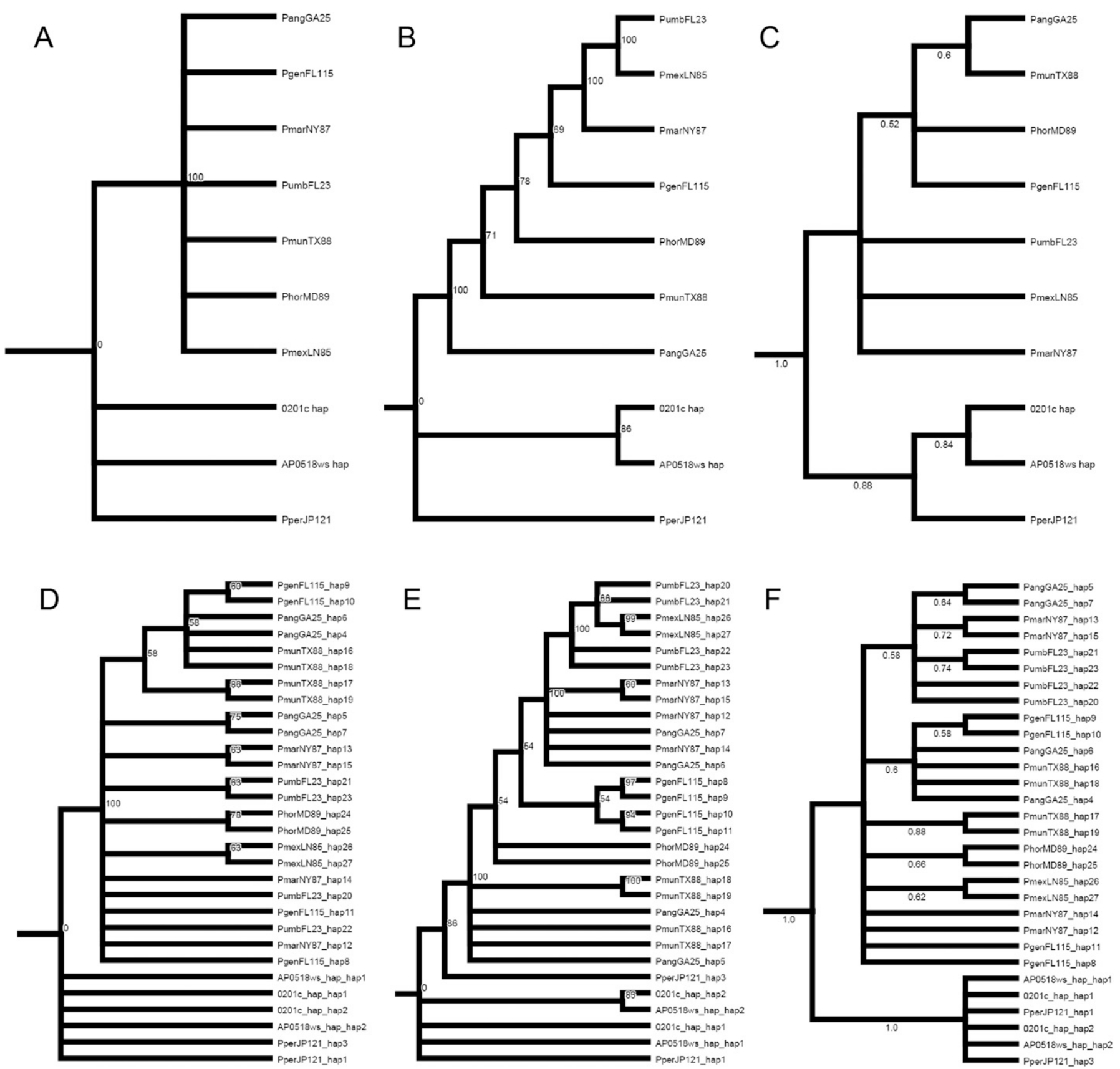

Supplemental Fig. 40. Majority rule consensus trees for FLC-FLF diploid sequence data using (A) maximum parsimony, (B) maximum parsimony (including gaps), and (C) maximum likelihood; and haplotypic sequence data using (D) maximum parsimony, (E) maximum parsimony (including gaps), and (F) maximum likelihood. Bootstrap values greater than $50 \%$ are described above the branches for maximum parsimony tree. Posterior probability values are described above the branches for maximum likehood tree. Trees are rooted with Prunus fasciculata (PfasCA131). Other species in the cladogram are Prunus americana (PameFL12), Prunus angustifolia (PangGA25), Prunus geniculata (PgenFL115), Prunus hortulana (PhorMD89), Prunus maritima (PmarNY87), Prunus mexicana (PmexLN85), Prunus munsoniana (PmunTX88), Prunus persica (PperJP121, 0201c_hap, AP0518ws_hap), Prunus pumila (PbesIL15), and Prunus umbellata (PumbFL23). 

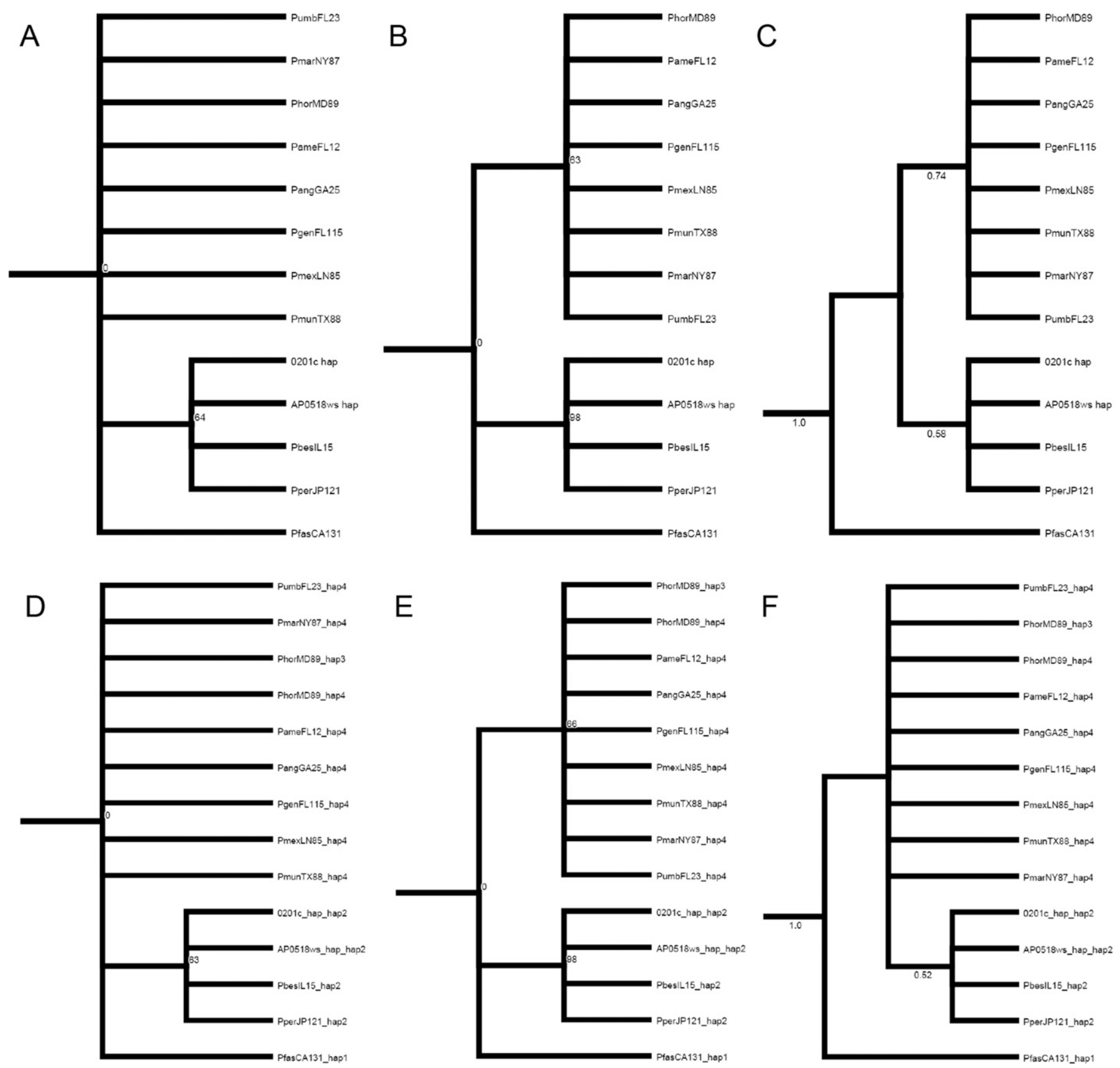

Supplemental Fig. 41. Majority rule consensus trees for FPF1 diploid sequence data using (A) maximum parsimony, (B) maximum parsimony (including gaps), and (C) maximum likelihood; and haplotypic sequence data using (D) maximum parsimony, (E) maximum parsimony (including gaps), and (F) maximum likelihood. Bootstrap values greater than $50 \%$ are described above the branches for maximum parsimony tree. Posterior probability values are described above the branches for maximum likehood tree. Trees are rooted with Prunus fasciculata (PfasCA131). Other species in the cladogram are Prunus americana (PameFL12), Prunus angustifolia (PangGA25), Prunus geniculata (PgenFL115), Prunus hortulana (PhorMD89), Prunus maritima (PmarNY87), Prunus mexicana (PmexLN85), Prunus munsoniana (PmunTX88), Prunus persica (PperJP121, 0201c_hap, AP0518ws_hap), Prunus pumila (PbesIL15), and Prunus umbellata (PumbFL23). 

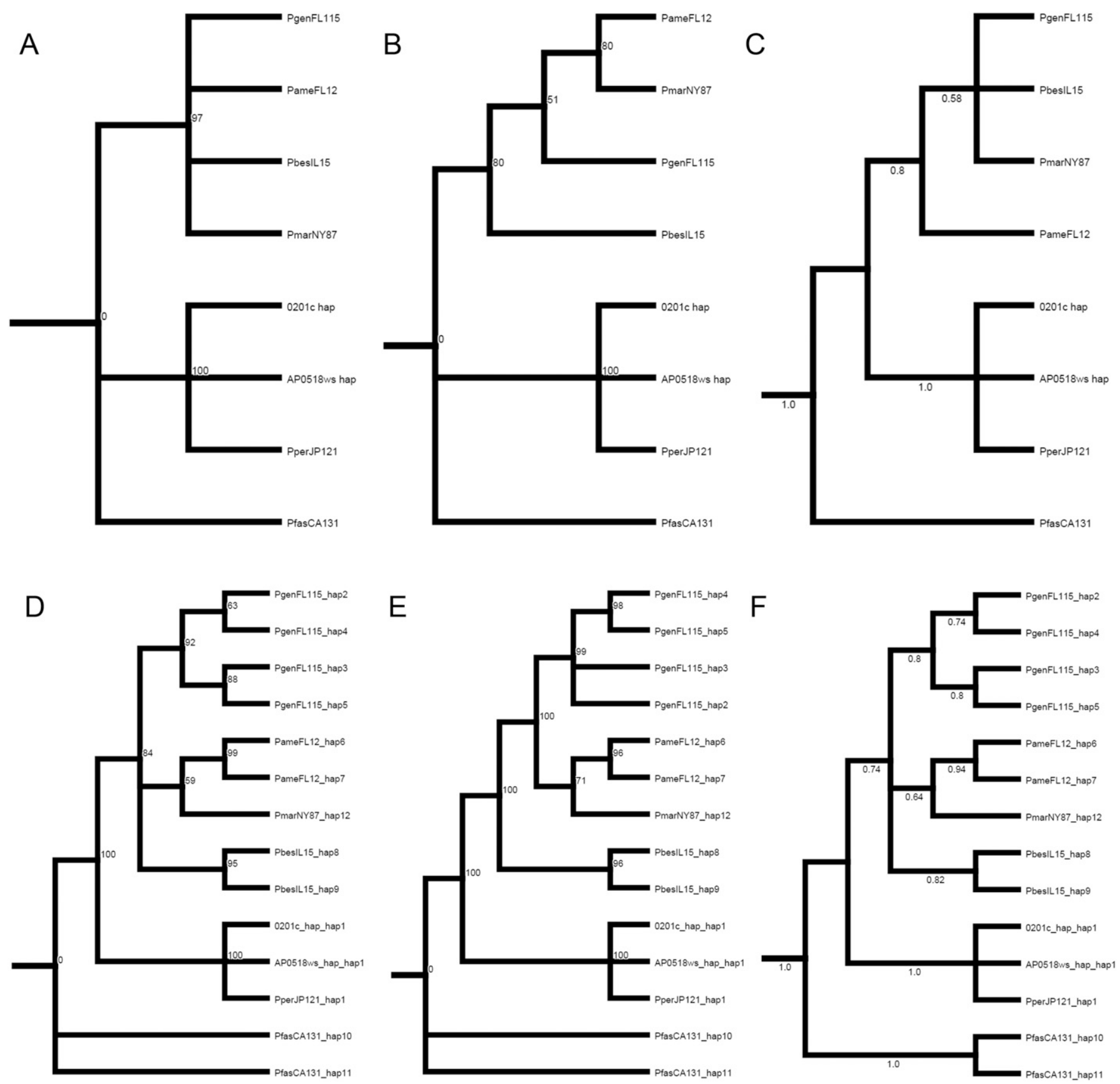

Supplemental Fig. 42. Majority rule consensus trees for FRIGIDA diploid sequence data using (A) maximum parsimony, (B) maximum parsimony (including gaps), and $(\mathbf{C})$ maximum likelihood; and haplotypic sequence data using (D) maximum parsimony, (E) maximum parsimony (including gaps), and (F) maximum likelihood. Bootstrap values greater than $50 \%$ are described above the branches for maximum parsimony tree. Posterior probability values are described above the branches for maximum likehood tree. Trees are rooted with Prunus fasciculata (PfasCA131). Other species in the cladogram are Prunus americana (PameFL12), Prunus angustifolia (PangGA25), Prunus geniculata (PgenFL115), Prunus hortulana (PhorMD89), Prunus maritima (PmarNY87), Prunus mexicana (PmexLN85), Prunus munsoniana (PmunTX88), Prunus persica (PperJP121, 0201c_hap, AP0518ws_hap), Prunus pumila (PbesIL15), and Prunus umbellata (PumbFL23). 

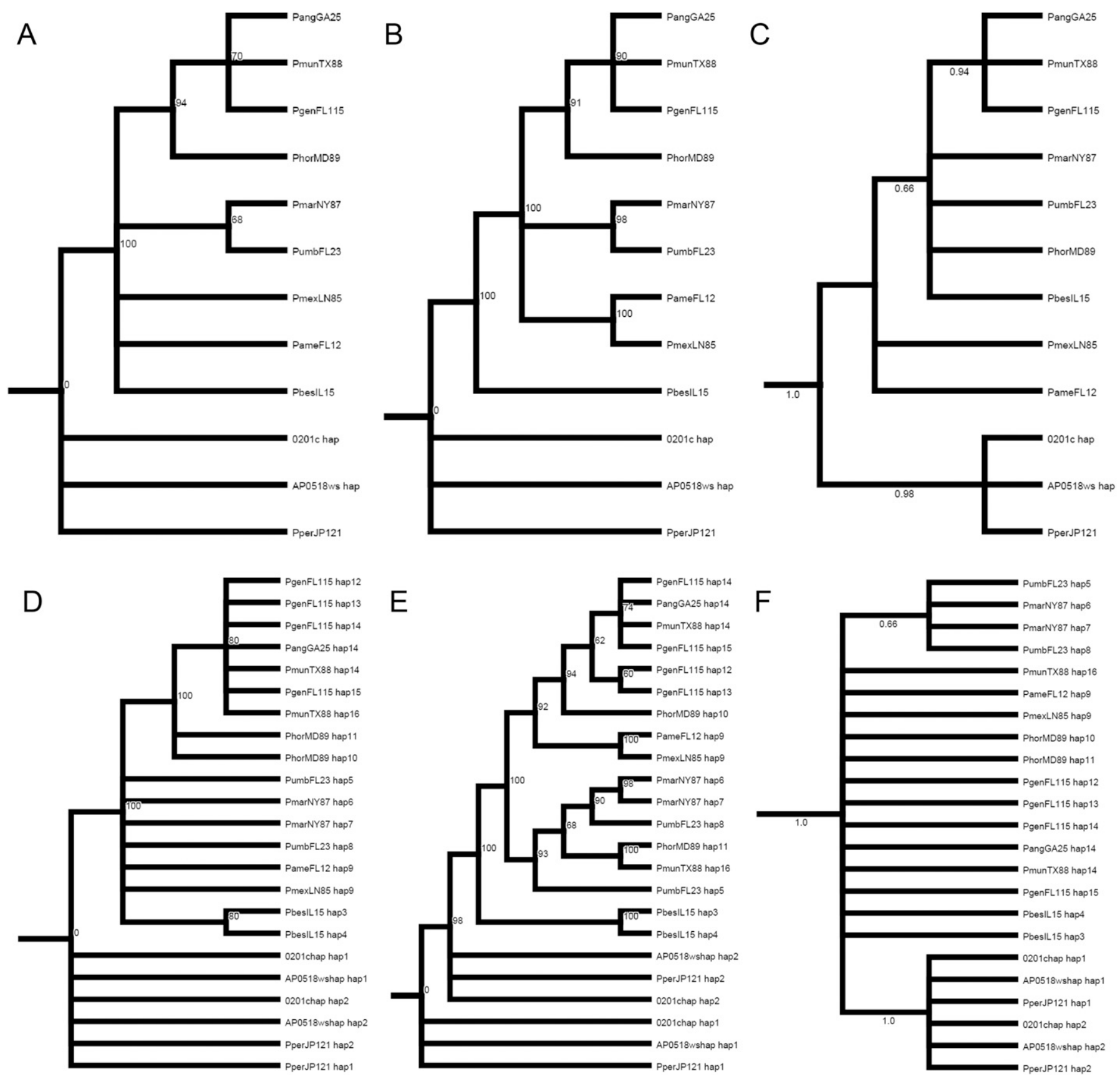

Supplemental Fig. 43. Majority rule consensus trees for FT-TSF diploid sequence data using (A) maximum parsimony, (B) maximum parsimony (including gaps), and (C) maximum likelihood; and haplotypic sequence data using (D) maximum parsimony, (E) maximum parsimony (including gaps), and (F) maximum likelihood. Bootstrap values greater than $50 \%$ are described above the branches for maximum parsimony tree. Posterior probability values are described above the branches for maximum likehood tree. Trees are rooted with Prunus fasciculata (PfasCA131). Other species in the cladogram are Prunus americana (PameFL12), Prunus angustifolia (PangGA25), Prunus geniculata (PgenFl115), Prunus hortulana (PhorMD89), Prunus maritima (PmarNY87), Prunus mexicana (PmexLN85), Prunus munsoniana (PmunTX88), Prunus persica (PperJP121, 0201c_hap, AP0518ws_hap), Prunus pumila (PbesIL15), and Prunus umbellata (PumbFL23). 

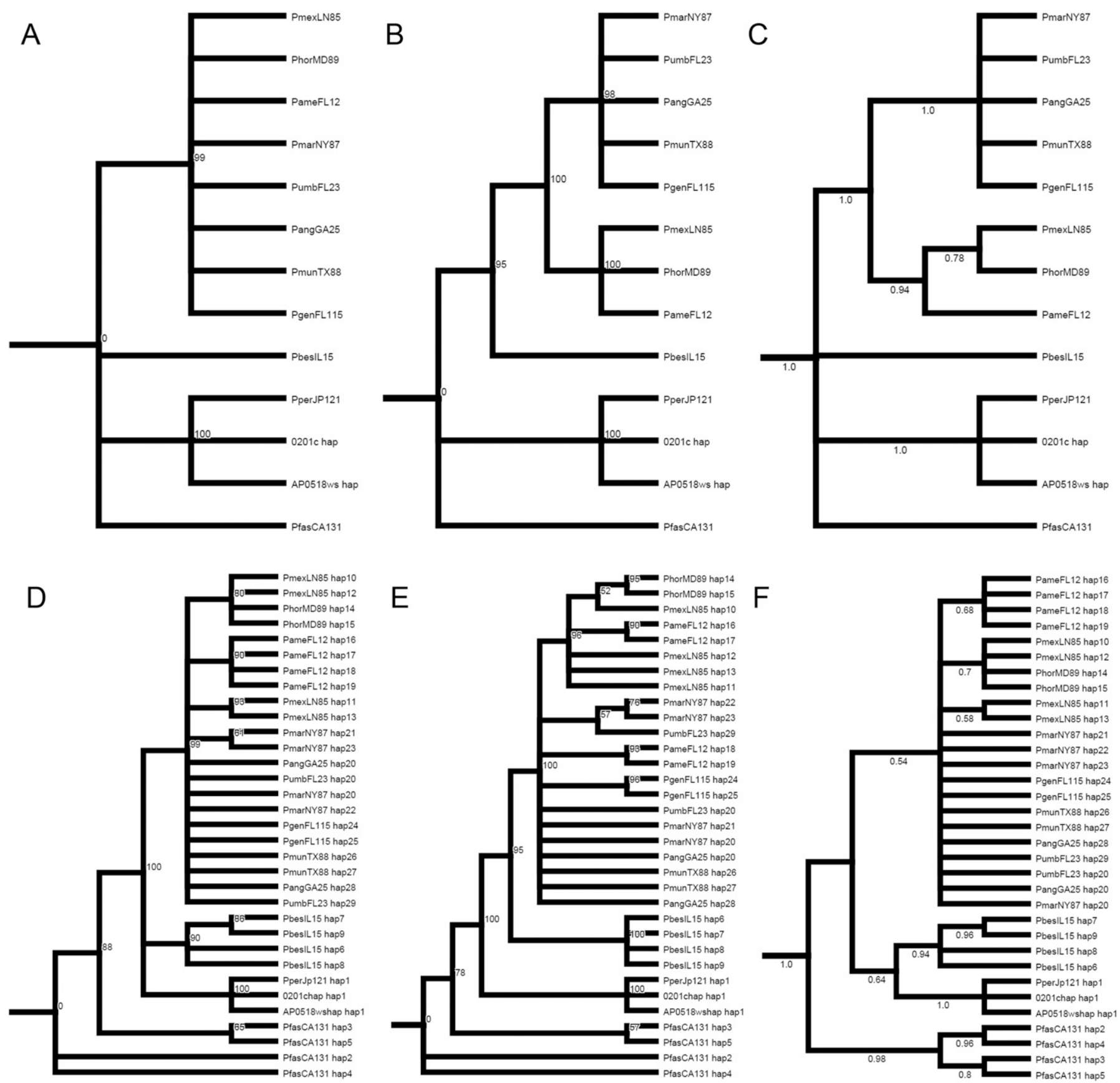

Supplemental Fig. 44. Majority rule consensus trees for GI-FB diploid sequence data using (A) maximum parsimony, (B) maximum parsimony (including gaps), and (C) maximum likelihood; and haplotypic sequence data using (D) maximum parsimony, (E) maximum parsimony (including gaps), and (F) maximum likelihood. Bootstrap values greater than $50 \%$ are described above the branches for maximum parsimony tree. Posterior probability values are described above the branches for maximum likehood tree. Trees are rooted with Prunus fasciculata (PfasCA131). Other species in the cladogram are Prunus americana (PameFL12), Prunus angustifolia (PangGA25), Prunus geniculata (PgenFL115), Prunus hortulana (PhorMD89), Prunus maritima (PmarNY87), Prunus mexicana (PmexLN85), Prunus munsoniana (PmunTX88), Prunus persica (PperJP121, 0201c_hap, AP0518ws_hap), Prunus pumila (PbesIL15), and Prunus umbellata (PumbFL23). 

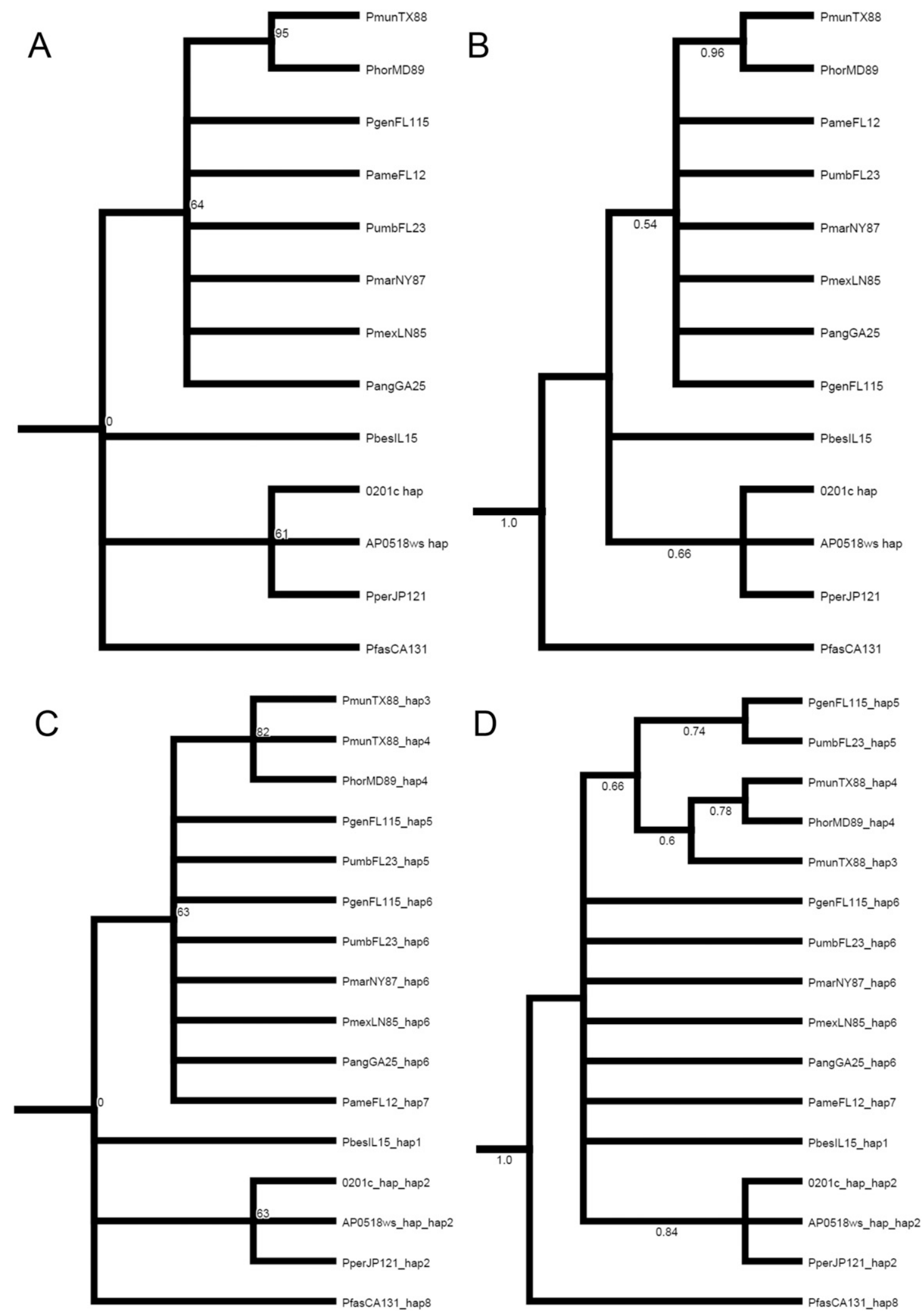

Supplemental Fig. 45. Majority rule consensus trees for HOS1 diploid sequence data using (A) maximum parsimony and (B) maximum likelihood; and haplotypic sequence data using (C) maximum parsimony and (D) maximum likelihood. Bootstrap values greater than $50 \%$ are described above the branches for maximum parsimony tree. Posterior probability values are described above the branches for maximum likehood tree. Trees are rooted with Prunus fasciculata (PfasCA131). Other species in the cladogram are Prunus americana (PameFL12), Prunus angustifolia (PangGA25), Prunus geniculata (PgenFL115), Prunus hortulana (PhorMD89), Prunus maritima (PmarNY87), Prunus mexicana (PmexLN85), Prunus munsoniana (PmunTX88), Prunus persica (PperJP121, 0201c_hap, AP0518ws_hap), Prunus pumila (PbesIL15), and Prunus umbellata (PumbFL23). 

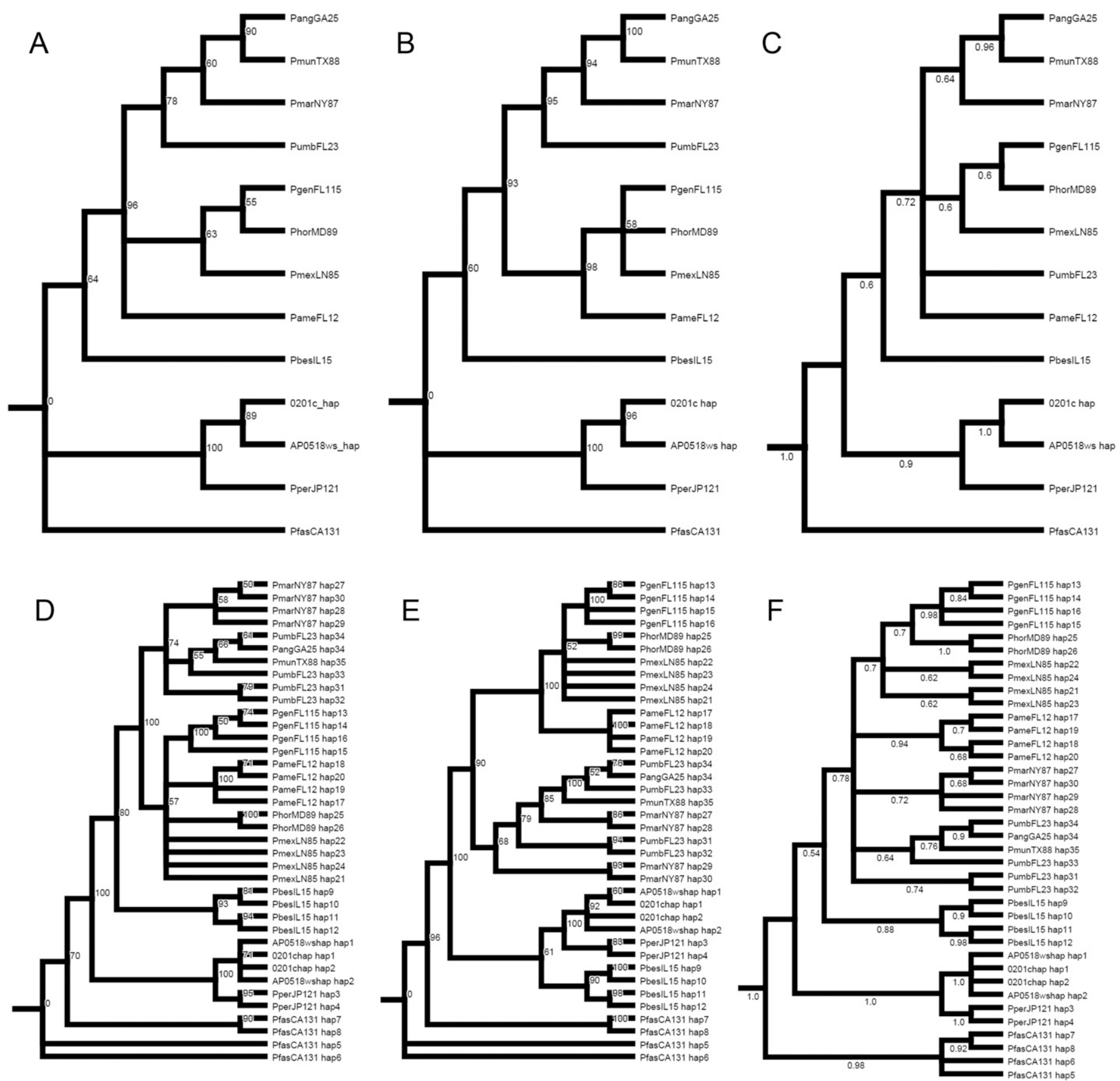

Supplemental Fig. 46. Majority rule consensus trees for LFY diploid sequence data using (A) maximum parsimony, (B) maximum parsimony (including gaps), and (C) maximum likelihood; and haplotypic sequence data using (D) maximum parsimony, (E) maximum parsimony (including gaps), and (F) maximum likelihood. Bootstrap values greater than $50 \%$ are described above the branches for maximum parsimony tree. Posterior probability values are described above the branches for maximum likehood tree. Trees are rooted with Prunus fasciculata (PfasCA131). Other species in the cladogram are Prunus americana (PameFL12), Prunus angustifolia (PangGA25), Prunus geniculata (PgenFL115), Prunus hortulana (PhorMD89), Prunus maritima (PmarNY87), Prunus mexicana (PmexLN85), Prunus munsoniana (PmunTX88), Prunus persica (PperJP121, 0201c_hap, AP0518ws_hap), Prunus pumila (PbesIL15), and Prunus umbellata (PumbFL23). 

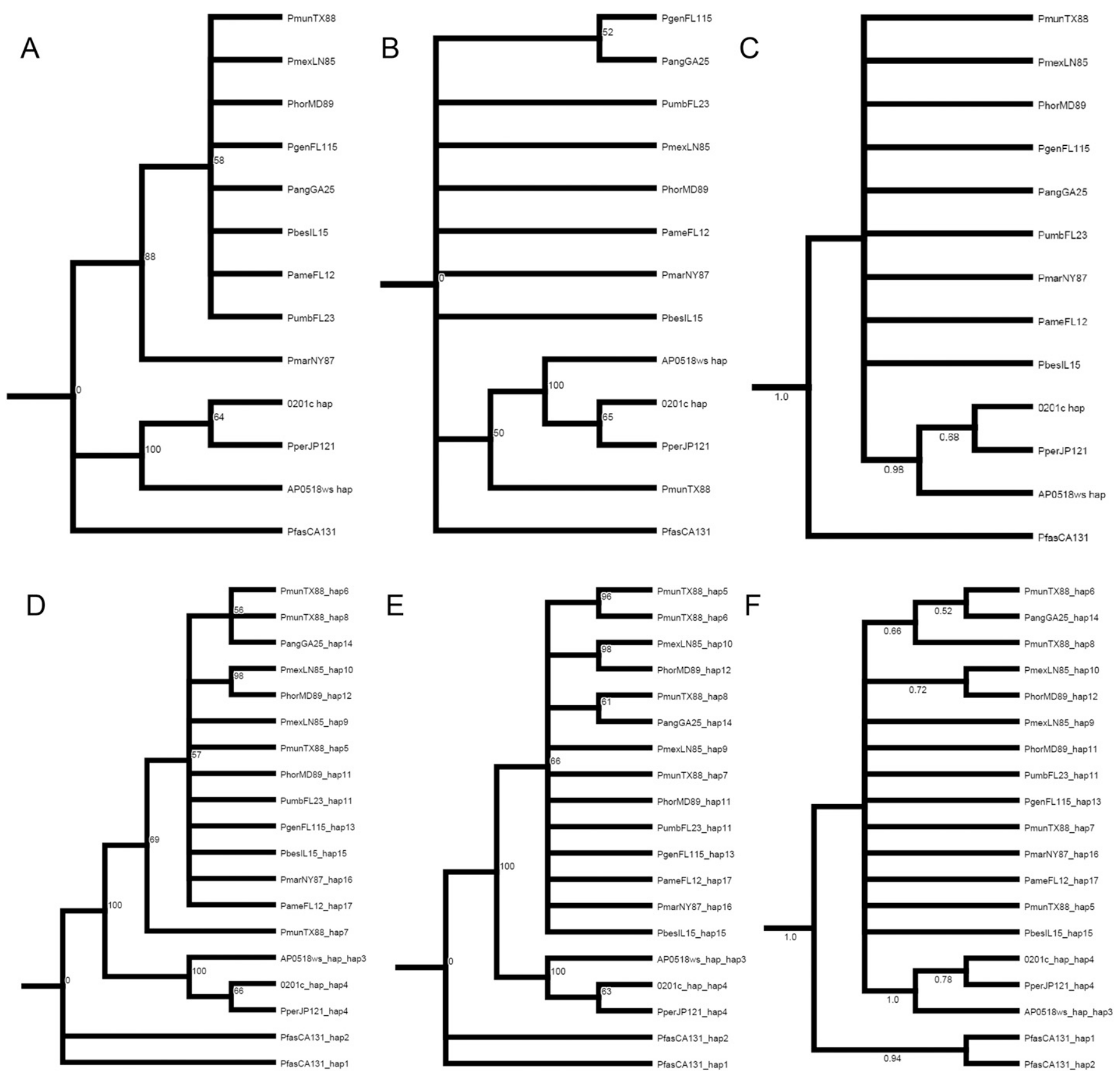

Supplemental Fig. 47. Majority rule consensus trees for MAF1-MAF3-AGL31 diploid sequence data using (A) maximum parsimony, (B) maximum parsimony (including gaps), and (C) maximum likelihood; and haplotypic sequence data using (D) maximum parsimony, (E) maximum parsimony (including gaps), and (F) maximum likelihood. Bootstrap values greater than $50 \%$ are described above the branches for maximum parsimony tree. Posterior probability values are described above the branches for maximum likehood tree. Trees are rooted with Prunus fasciculata (PfasCA131). Other species in the cladogram are Prunus americana (PameFL12), Prunus angustifolia (PangGA25), Prunus geniculata (PgenFL115), Prunus hortulana (PhorMD89), Prunus maritima (PmarNY87), Prunus mexicana (PmexLN85), Prunus munsoniana (PmunTX88), Prunus persica (PperJP121, 0201c_hap, AP0518ws_hap), Prunus pumila (PbesIL15), and Prunus umbellata (PumbFL23). 

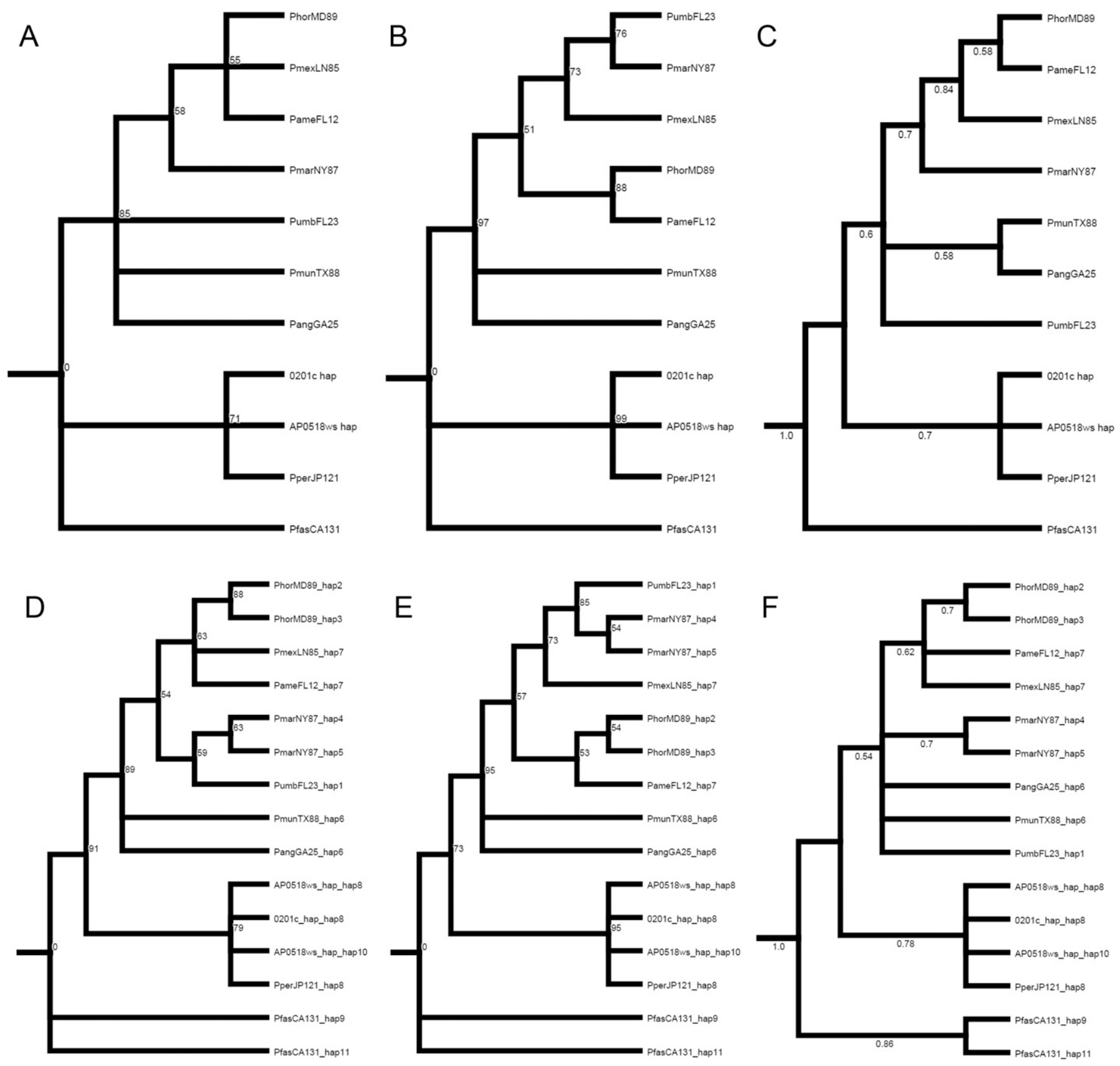

Supplemental Fig. 48. Majority rule consensus trees for MAF2A diploid sequence data using (A) maximum parsimony, (B) maximum parsimony (including gaps), and $(\mathbf{C})$ maximum likelihood; and haplotypic sequence data using $(\mathbf{D})$ maximum parsimony, (E) maximum parsimony (including gaps), and (F) maximum likelihood. Bootstrap values greater than $50 \%$ are described above the branches for maximum parsimony tree. Posterior probability values are described above the branches for maximum likehood tree. Trees are rooted with Prunus fasciculata (PfasCA131). Other species in the cladogram are Prunus americana (PameFL12), Prunus angustifolia (PangGA25), Prunus geniculata (PgenFL115), Prunus hortulana (PhorMD89), Prunus maritima (PmarNY87), Prunus mexicana (PmexLN85), Prunus munsoniana (PmunTX88), Prunus persica (PperJP121, 0201c_hap, AP0518ws_hap), Prunus pumila (PbesIL15), and Prunus umbellata (PumbFL23). 

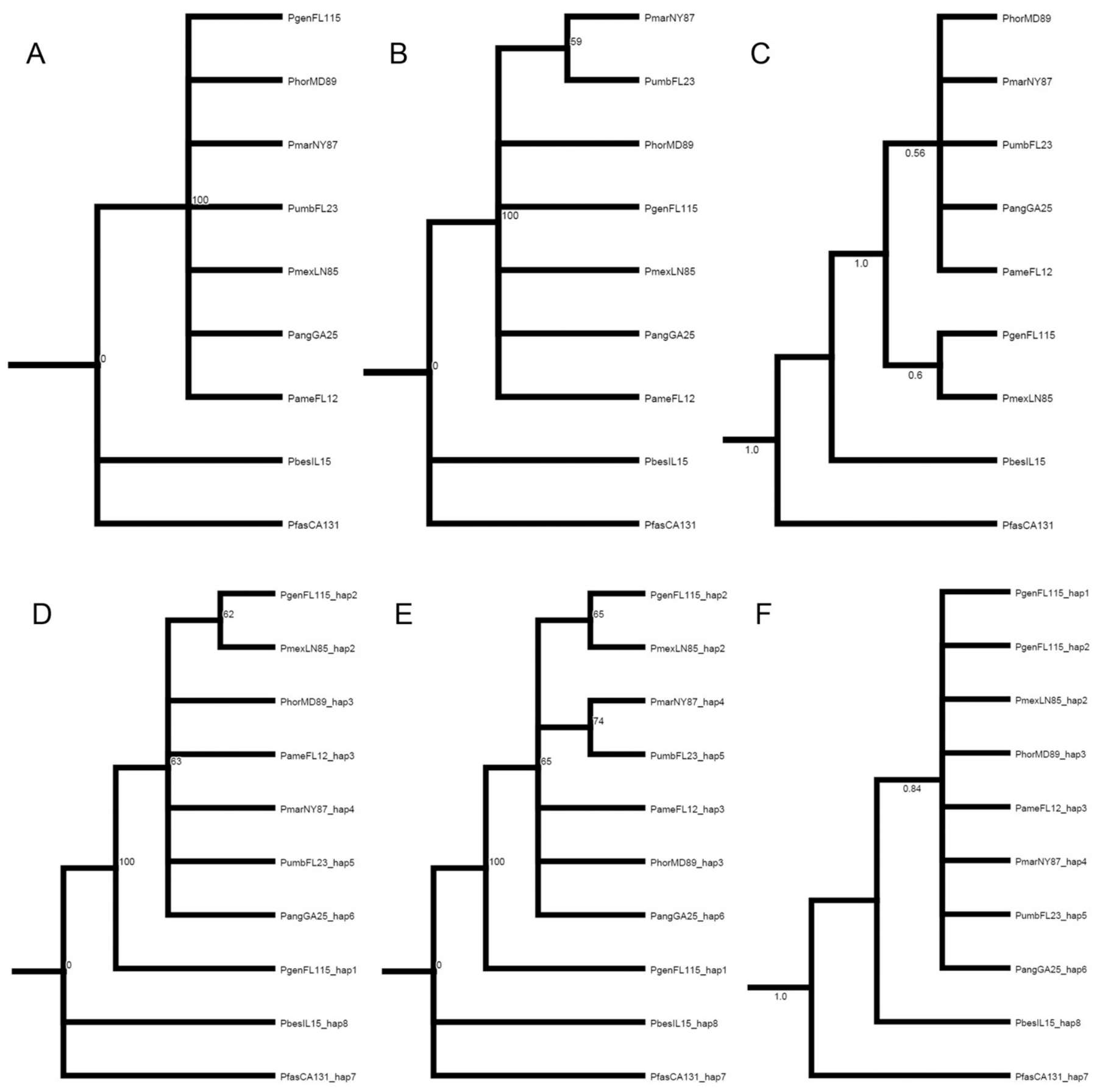

Supplemental Fig. 49. Majority rule consensus trees for MAF2B diploid sequence data using (A) maximum parsimony, (B) maximum parsimony (including gaps), and (C) maximum likelihood; and haplotypic sequence data using (D) maximum parsimony, (E) maximum parsimony (including gaps), and (F) maximum likelihood. Bootstrap values greater than $50 \%$ are described above the branches for maximum parsimony tree. Posterior probability values are described above the branches for maximum likehood tree. Trees are rooted with Prunus fasciculata (PfasCA131). Other species in the cladogram are Prunus americana (PameFL12), Prunus angustifolia (PangGA25), Prunus geniculata (PgenFL115), Prunus hortulana (PhorMD89), Prunus maritima (PmarNY87), Prunus mexicana (PmexLN85), Prunus munsoniana (PmunTX88), Prunus pumila (PbesIL15), and Prunus umbellata (PumbFL23). 

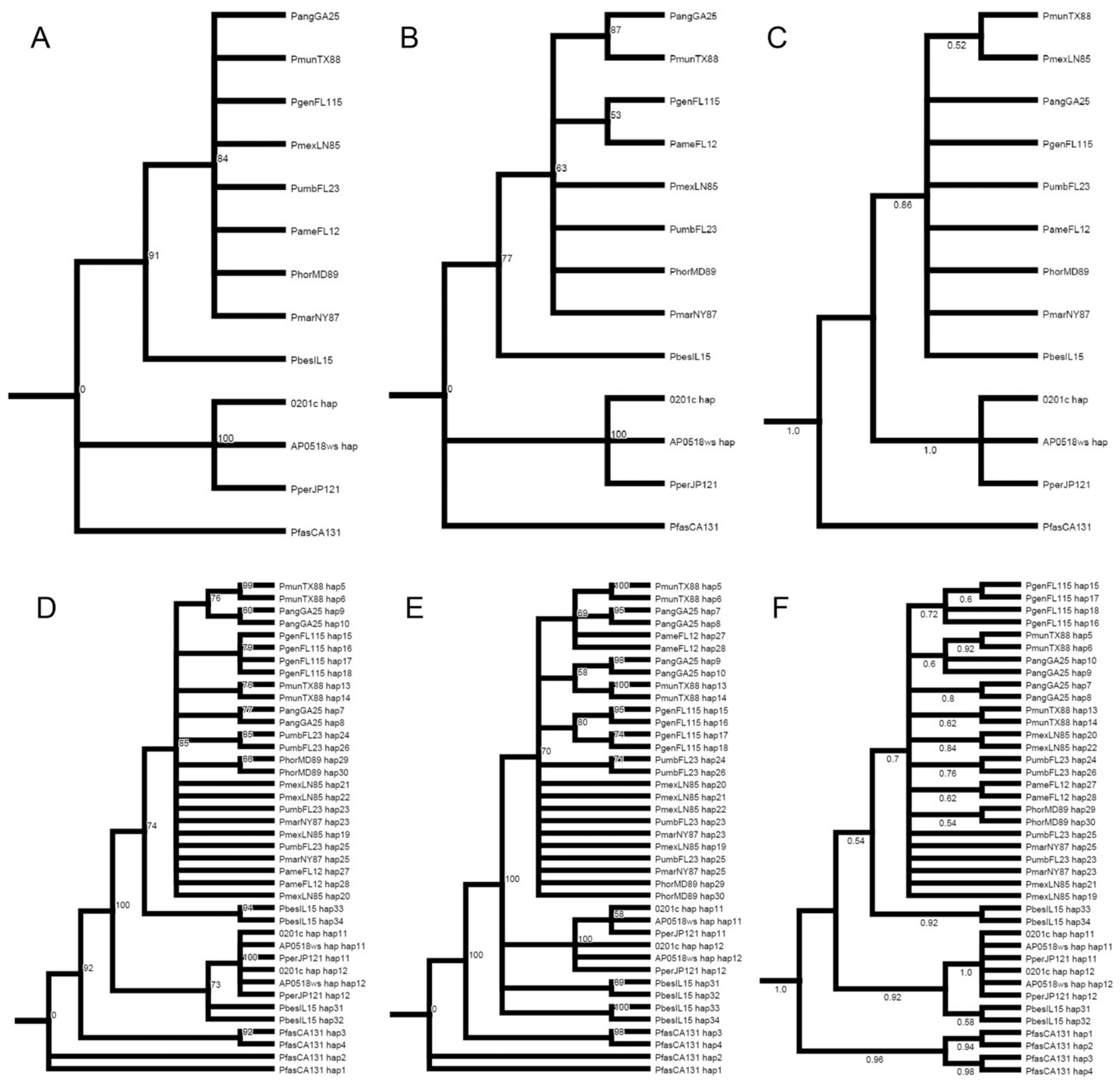

Supplemental Fig. 50. Majority rule consensus trees for MAF4 diploid sequence data using (A) maximum parsimony, (B) maximum parsimony (including gaps), and $(\mathbf{C})$ maximum likelihood; and haplotypic sequence data using (D) maximum parsimony, (E) maximum parsimony (including gaps), and (F) maximum likelihood. Bootstrap values greater than $50 \%$ are described above the branches for maximum parsimony tree. Posterior probability values are described above the branches for maximum likehood tree. Trees are rooted with Prunus fasciculata (PfasCA131). Other species in the cladogram are Prunus americana (PameFL12), Prunus angustifolia (PangGA25), Prunus geniculata (PgenFL115), Prunus hortulana (PhorMD89), Prunus maritima (PmarNY87), Prunus mexicana (PmexLN85), Prunus munsoniana (PmunTX88), Prunus persica (PperJP121, 0201c_hap, AP0518ws_hap), Prunus pumila (PbesIL15), and Prunus umbellata (PumbFL23). 


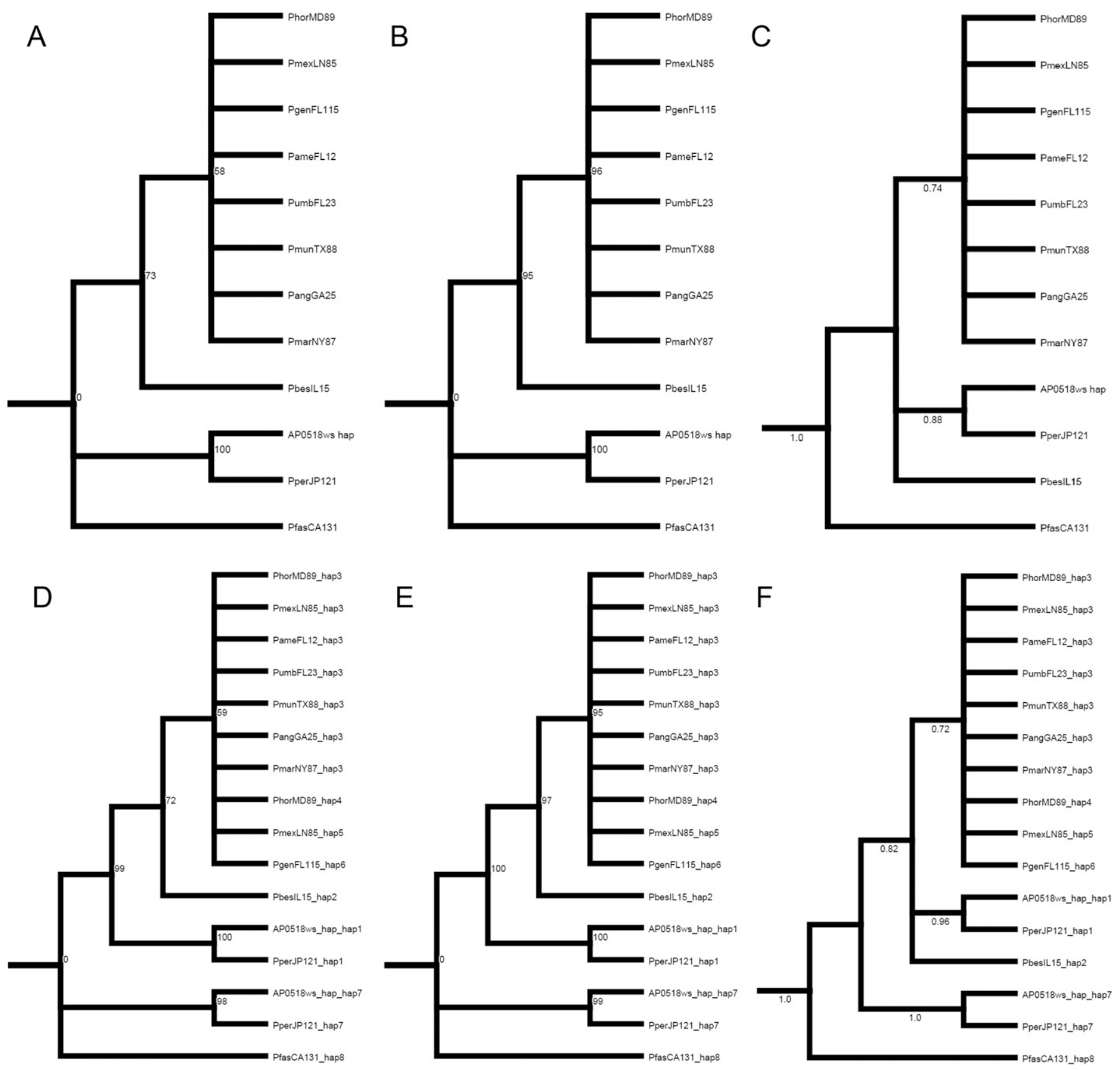

Supplemental Fig. 51. Majority rule consensus trees for MAF5 diploid sequence data using (A) maximum parsimony, (B) maximum parsimony (including gaps), and (C) maximum likelihood; and haplotypic sequence data using (D) maximum parsimony, (E) maximum parsimony (including gaps), and (F) maximum likelihood. Bootstrap values greater than $50 \%$ are described above the branches for maximum parsimony tree. Posterior probability values are described above the branches for maximum likehood tree. Trees are rooted with Prunus fasciculata (PfasCA131). Other species in the cladogram are Prunus americana (PameFL12), Prunus angustifolia (PangGA25), Prunus geniculata (PgenFL115), Prunus hortulana (PhorMD89), Prunus maritima (PmarNY87), Prunus mexicana (PmexLN85), Prunus munsoniana (PmunTX88), Prunus persica (PperJP121, 0201c_hap, AP0518ws_hap), Prunus pumila (PbesIL15), and Prunus umbellata (PumbFL23). 

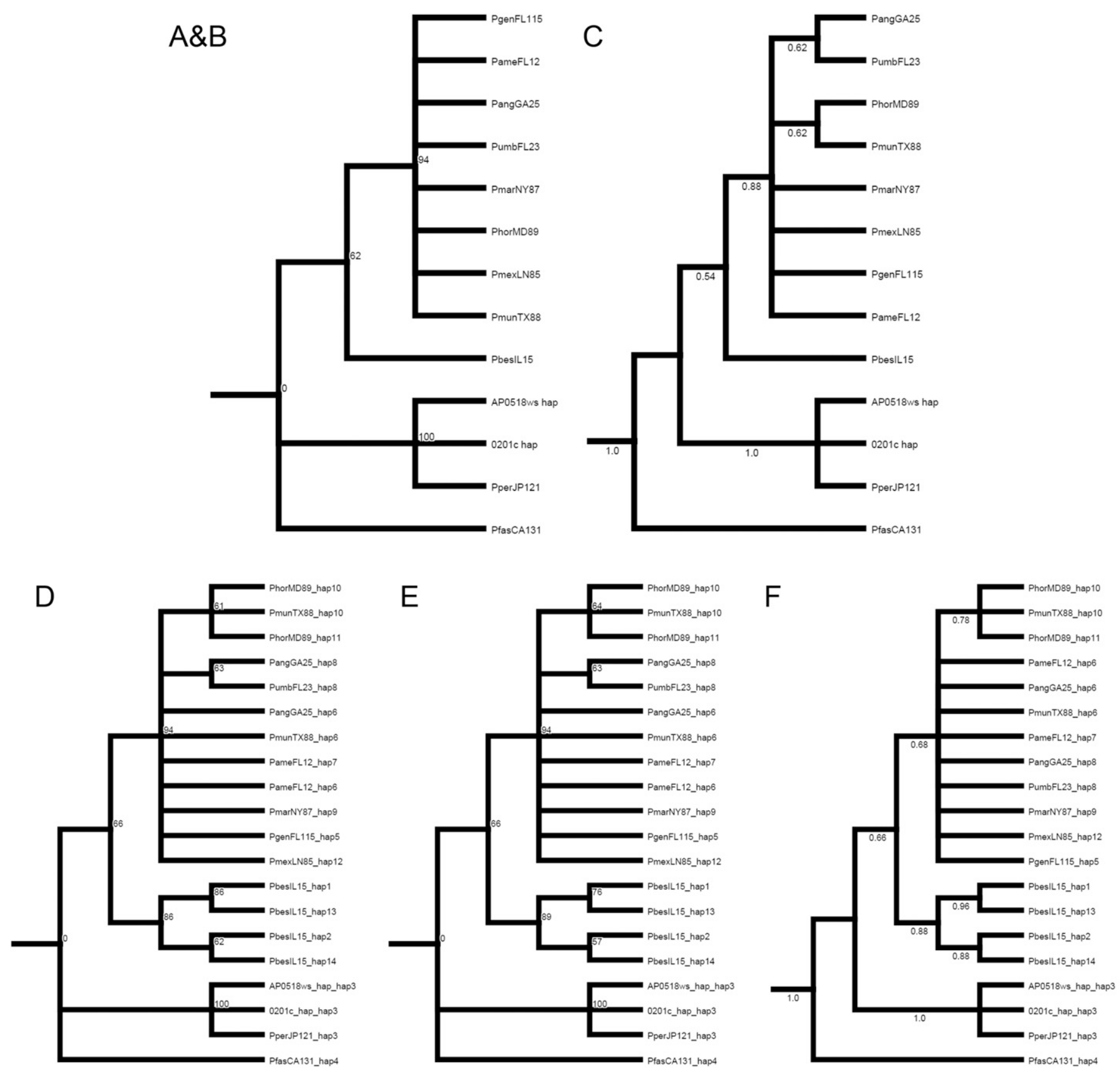

Supplemental Fig. 52. Majority rule consensus trees for MFT diploid sequence data using (A) maximum parsimony, (B) maximum parsimony (including gaps), and (C) maximum likelihood; and haplotypic sequence data using (D) maximum parsimony, (E) maximum parsimony (including gaps), and (F) maximum likelihood. Bootstrap values greater than $50 \%$ are described above the branches for maximum parsimony tree. Posterior probability values are described above the branches for maximum likehood tree. Trees are rooted with Prunus fasciculata (PfasCA131). Other species in the cladogram are Prunus americana (PameFL12), Prunus angustifolia (PangGA25), Prunus geniculata (PgenFL115), Prunus hortulana (PhorMD89), Prunus maritima (PmarNY87), Prunus mexicana (PmexLN85), Prunus munsoniana (PmunTX88), Prunus persica (PperJP121, 0201c_hap, AP0518ws_hap), Prunus pumila (PbesIL15), and Prunus umbellata (PumbFL23). 

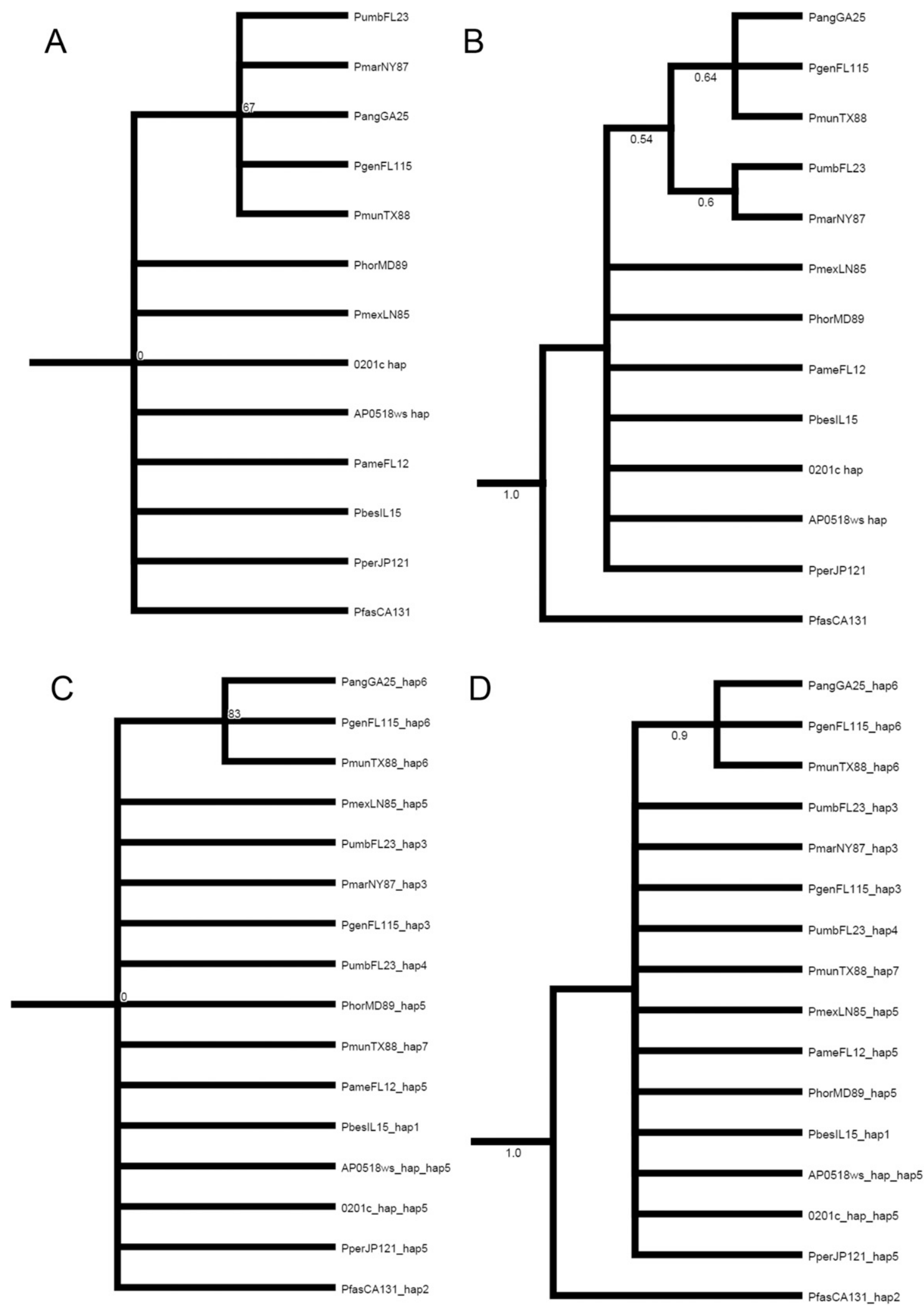

Supplemental Fig. 53. Majority rule consensus trees for PHYA diploid sequence data using (A) maximum parsimony and (B) maximum likelihood; and haplotypic sequence data using (C) maximum parsimony and (D) maximum likelihood. Bootstrap values greater than 50\% are described above the branches for maximum parsimony tree. Posterior probability values are described above the branches for maximum likehood tree. Trees are rooted with Prunus fasciculata (PfasCA131). Other species in the cladogram are Prunus americana (PameFL12), Prunus angustifolia (PangGA25), Prunus geniculata (PgenFL115), Prunus hortulana (PhorMD89), Prunus maritima (PmarNY87), Prunus mexicana (PmexLN85), Prunus munsoniana (PmunTX88), Prunus persica (PperJP121, 0201c_hap, AP0518ws hap), Prunus pumila (PbesIL15), and Prunus umbellata (PumbFL23). 

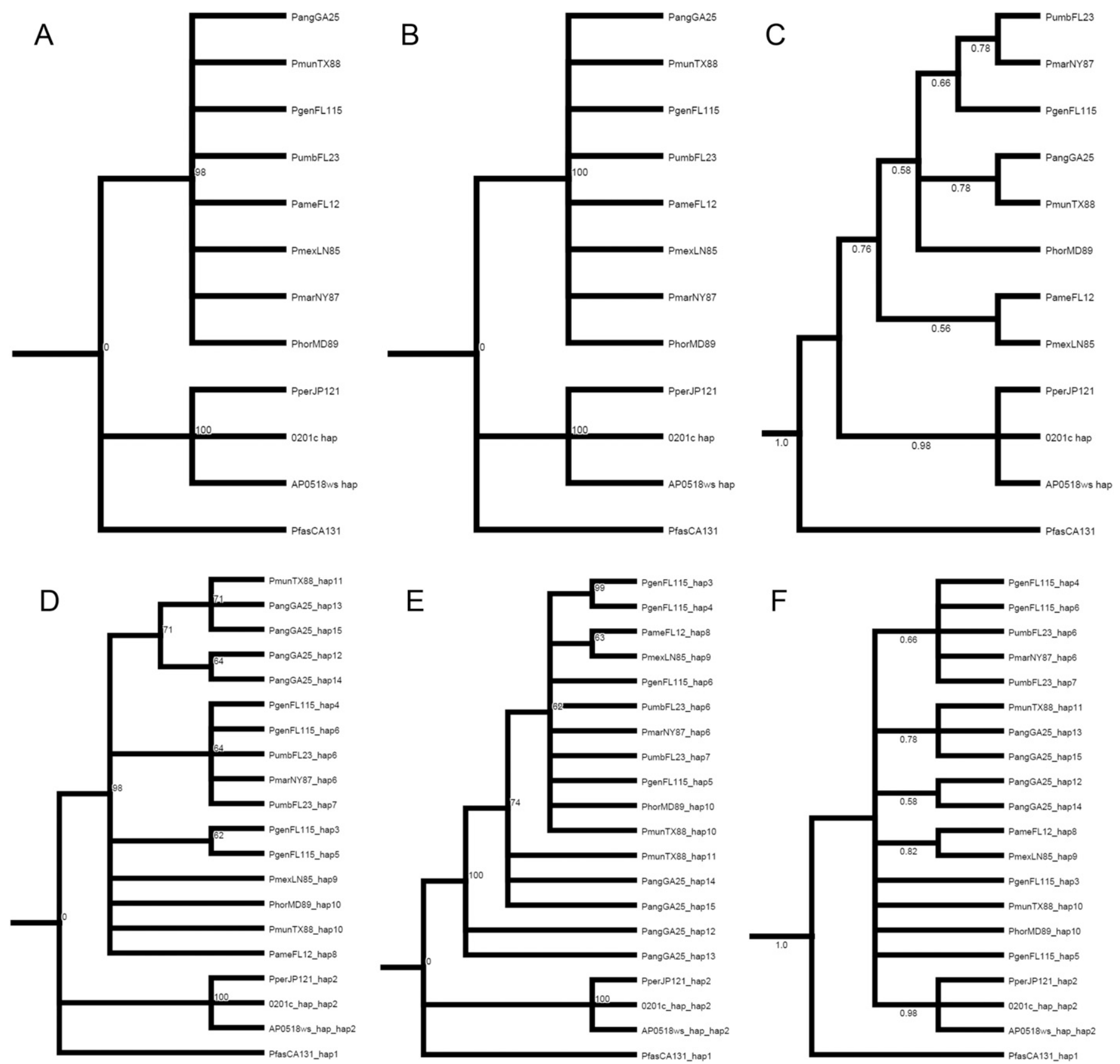

Supplemental Fig. 54. Majority rule consensus trees for PHYB-PHYD diploid sequence data using (A) maximum parsimony, (B) maximum parsimony (including gaps), and (C) maximum likelihood; and haplotypic sequence data using (D) maximum parsimony, (E) maximum parsimony (including gaps), and (F) maximum likelihood. Bootstrap values greater than $50 \%$ are described above the branches for maximum parsimony tree. Posterior probability values are described above the branches for maximum likehood tree. Trees are rooted with Prunus fasciculata (PfasCA131). Other species in the cladogram are Prunus americana (PameFL12), Prunus angustifolia (PangGA25), Prunus geniculata (PgenFL115), Prunus hortulana (PhorMD89), Prunus maritima (PmarNY87), Prunus mexicana (PmexLN85), Prunus munsoniana (PmunTX88), Prunus persica (PperJP121, 0201c_hap, AP0518ws_hap), Prunus pumila (PbesIL15), and Prunus umbellata (PumbFL23). 

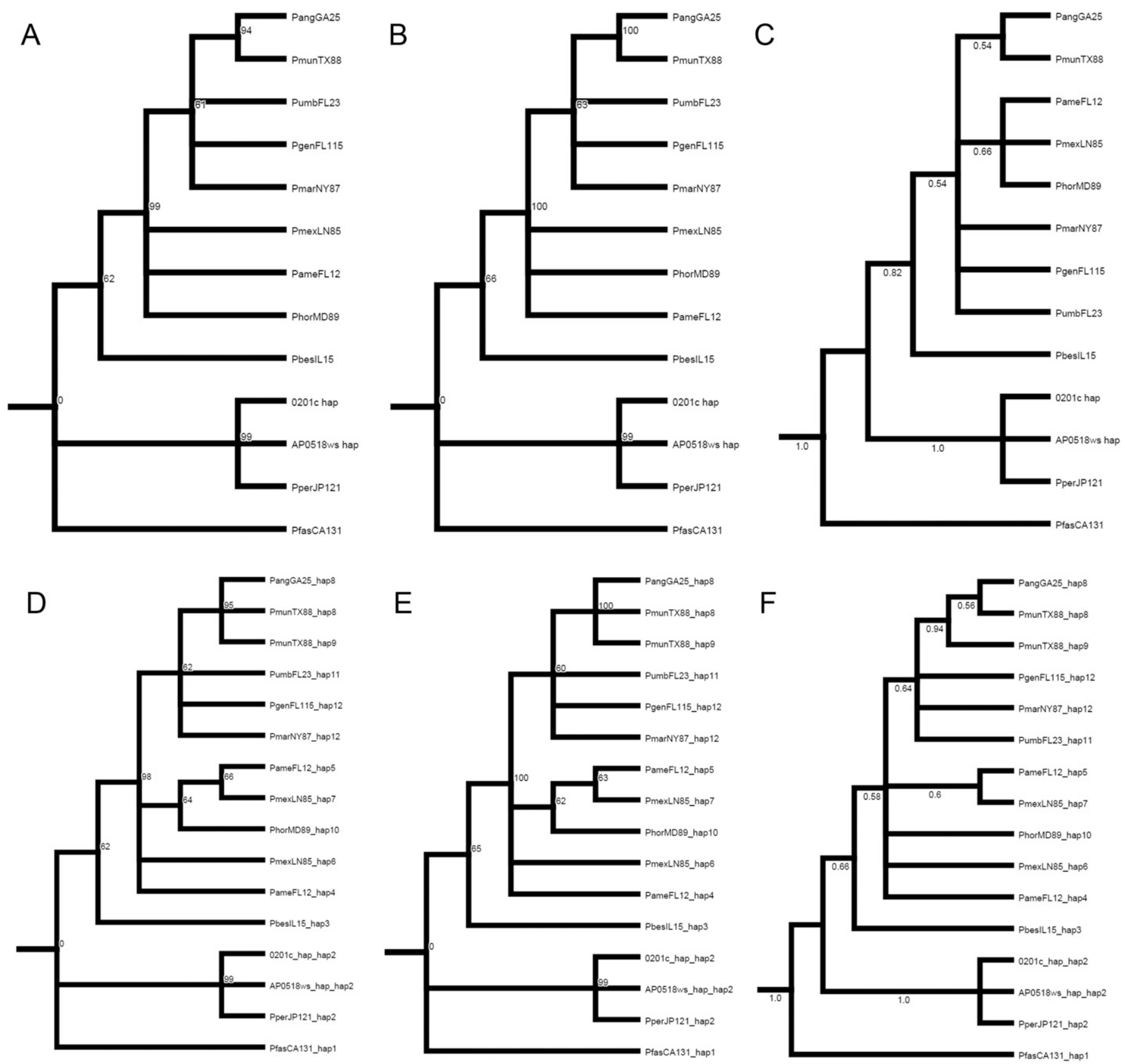

Supplemental Fig. 55. Majority rule consensus trees for PHYE diploid sequence data using (A) maximum parsimony, (B) maximum parsimony (including gaps), and (C) maximum likelihood; and haplotypic sequence data using (D) maximum parsimony, (E) maximum parsimony (including gaps), and (F) maximum likelihood. Bootstrap values greater than $50 \%$ are described above the branches for maximum parsimony tree. Posterior probability values are described above the branches for maximum likehood tree. Trees are rooted with Prunus fasciculata (PfasCA131). Other species in the cladogram are Prunus americana (PameFL12), Prunus angustifolia (PangGA25), Prunus geniculata (PgenFL115), Prunus hortulana (PhorMD89), Prunus maritima (PmarNY87), Prunus mexicana (PmexLN85), Prunus munsoniana (PmunTX88), Prunus persica (PperJP121, 0201c_hap, AP0518ws_hap), Prunus pumila (PbesIL15), and Prunus umbellata (PumbFL23). 

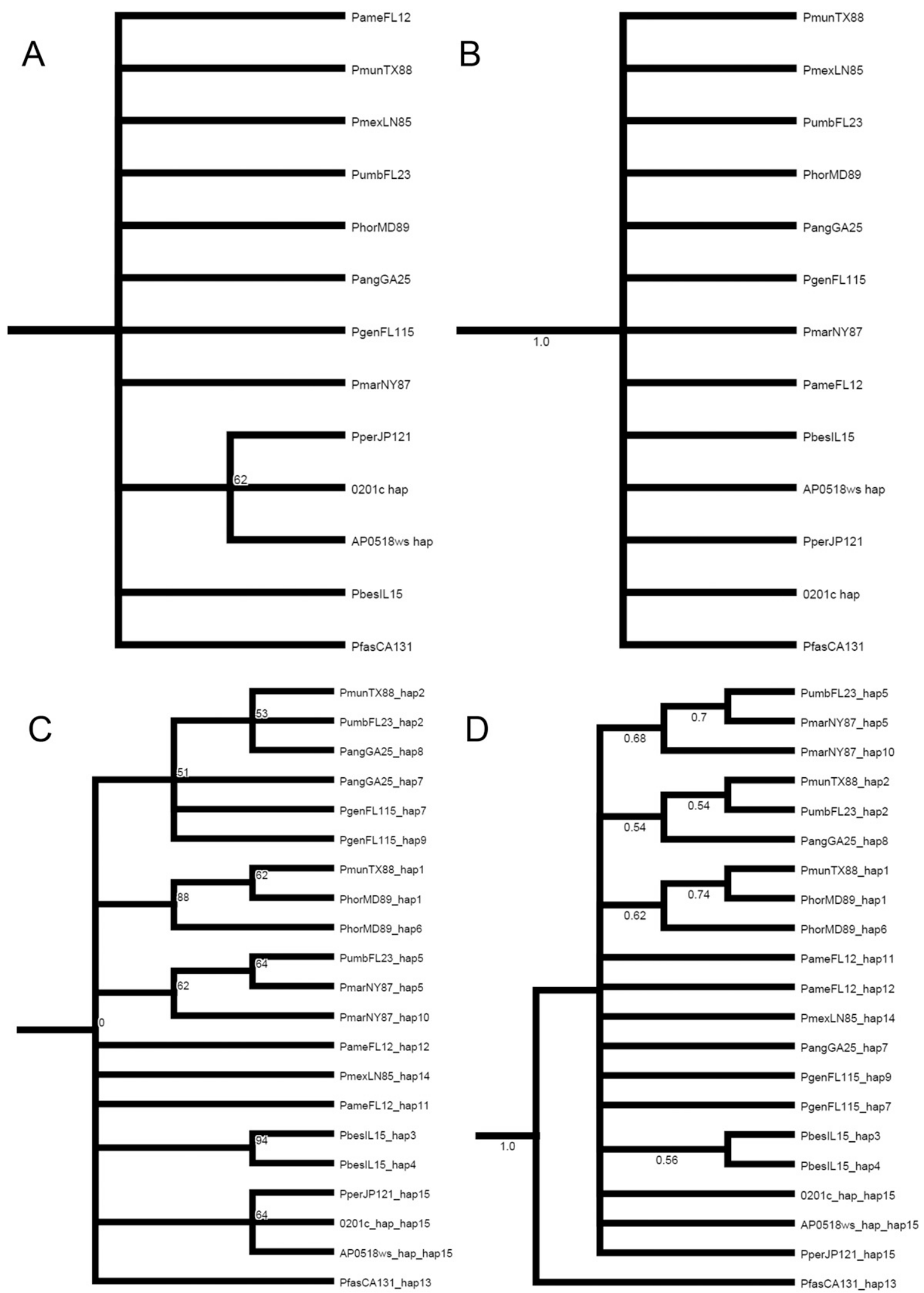

Supplemental Fig. 56. Majority rule consensus trees for RGA-RGA1 diploid sequence data using (A) maximum parsimony and (B) maximum likelihood; and haplotypic sequence data using $(\mathbf{C})$ maximum parsimony and (D) maximum likelihood. Bootstrap values greater than $50 \%$ are described above the branches for maximum parsimony tree. Posterior probability values are described above the branches for maximum likehood tree. Trees are rooted with Prunus fasciculata (PfasCA131). Other species in the cladogram are Prunus americana (PameFL12), Prunus angustifolia (PangGA25), Prunus geniculata (PgenFL115), Prunus hortulana (PhorMD89), Prunus maritima (PmarNY87), Prunus mexicana (PmexLN85), Prunus munsoniana (PmunTX88), Prunus persica (PperJP121, 0201c_hap, AP0518ws_hap), Prunus pumila (PbesIL15), and Prunus umbellata (PumbFL23). 

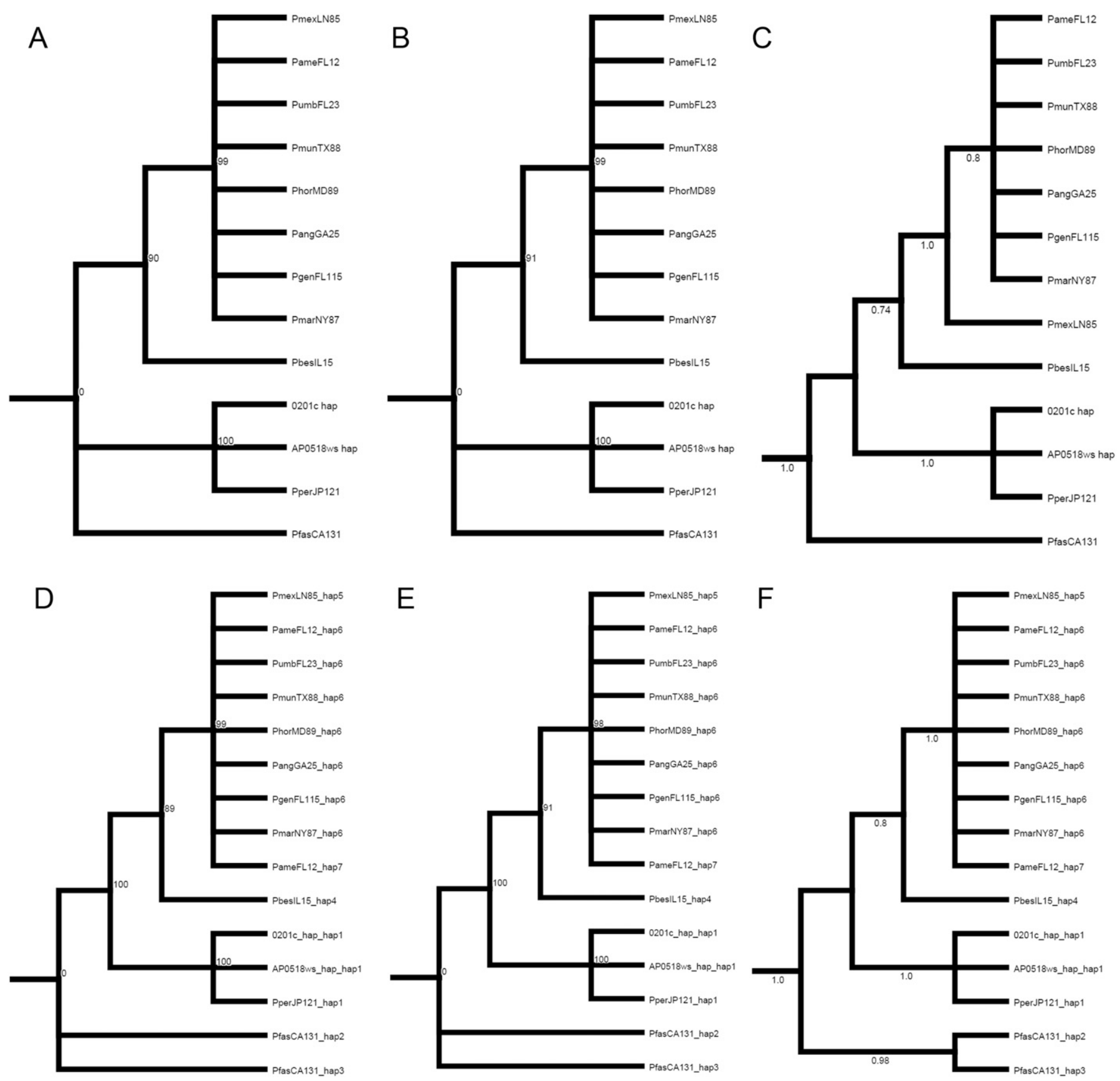

Supplemental Fig. 57. Majority rule consensus trees for RGL1-RGL2-RGL3 diploid sequence data using (A) maximum parsimony, (B) maximum parsimony (including gaps), and (C) maximum likelihood; and haplotypic sequence data using (D) maximum parsimony, (E) maximum parsimony (including gaps), and (F) maximum likelihood. Bootstrap values greater than $50 \%$ are described above the branches for maximum parsimony tree. Posterior probability values are described above the branches for maximum likehood tree. Trees are rooted with Prunus fasciculata (PfasCA131). Other species in the cladogram are Prunus americana (PameFL12), Prunus angustifolia (PangGA25), Prunus geniculata (PgenFL115), Prunus hortulana (PhorMD89), Prunus maritima (PmarNY87), Prunus mexicana (PmexLN85), Prunus munsoniana (PmunTX88), Prunus persica (PperJP121, 0201c_hap, AP0518ws_hap), Prunus pumila (PbesIL15), and Prunus umbellata (PumbFL23). 

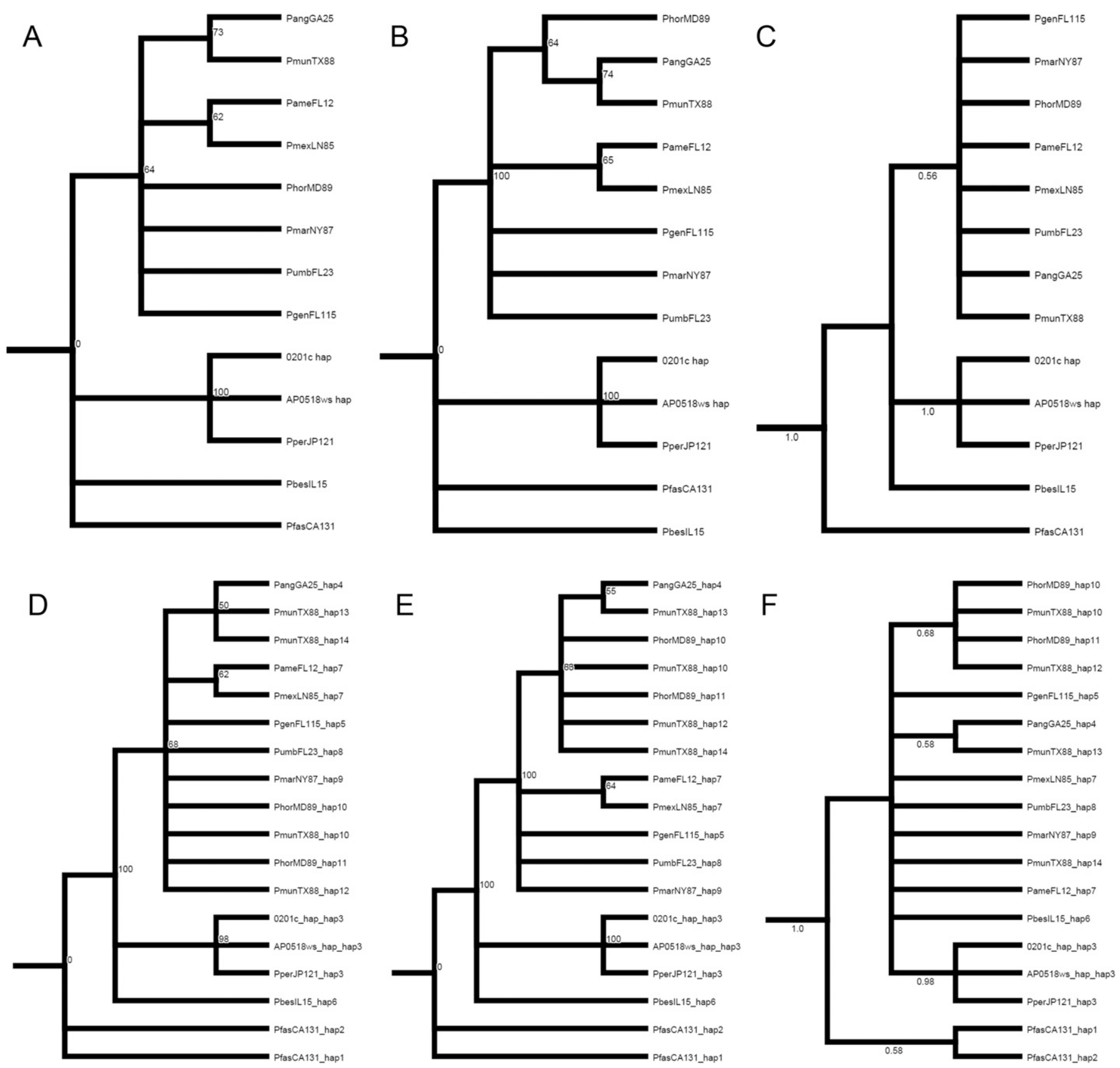

Supplemental Fig. 58. Majority rule consensus trees for SPY diploid sequence data using (A) maximum parsimony, (B) maximum parsimony (including gaps), and (C) maximum likelihood; and haplotypic sequence data using (D) maximum parsimony, (E) maximum parsimony (including gaps), and (F) maximum likelihood. Bootstrap values greater than $50 \%$ are described above the branches for maximum parsimony tree. Posterior probability values are described above the branches for maximum likehood tree. Trees are rooted with Prunus fasciculata (PfasCA131). Other species in the cladogram are Prunus americana (PameFL12), Prunus angustifolia (PangGA25), Prunus geniculata (PgenFL115), Prunus hortulana (PhorMD89), Prunus maritima (PmarNY87), Prunus mexicana (PmexLN85), Prunus munsoniana (PmunTX88), Prunus persica (PperJP121, 0201c_hap, AP0518ws_hap), Prunus pumila (PbesIL15), and Prunus umbellata (PumbFL23). 

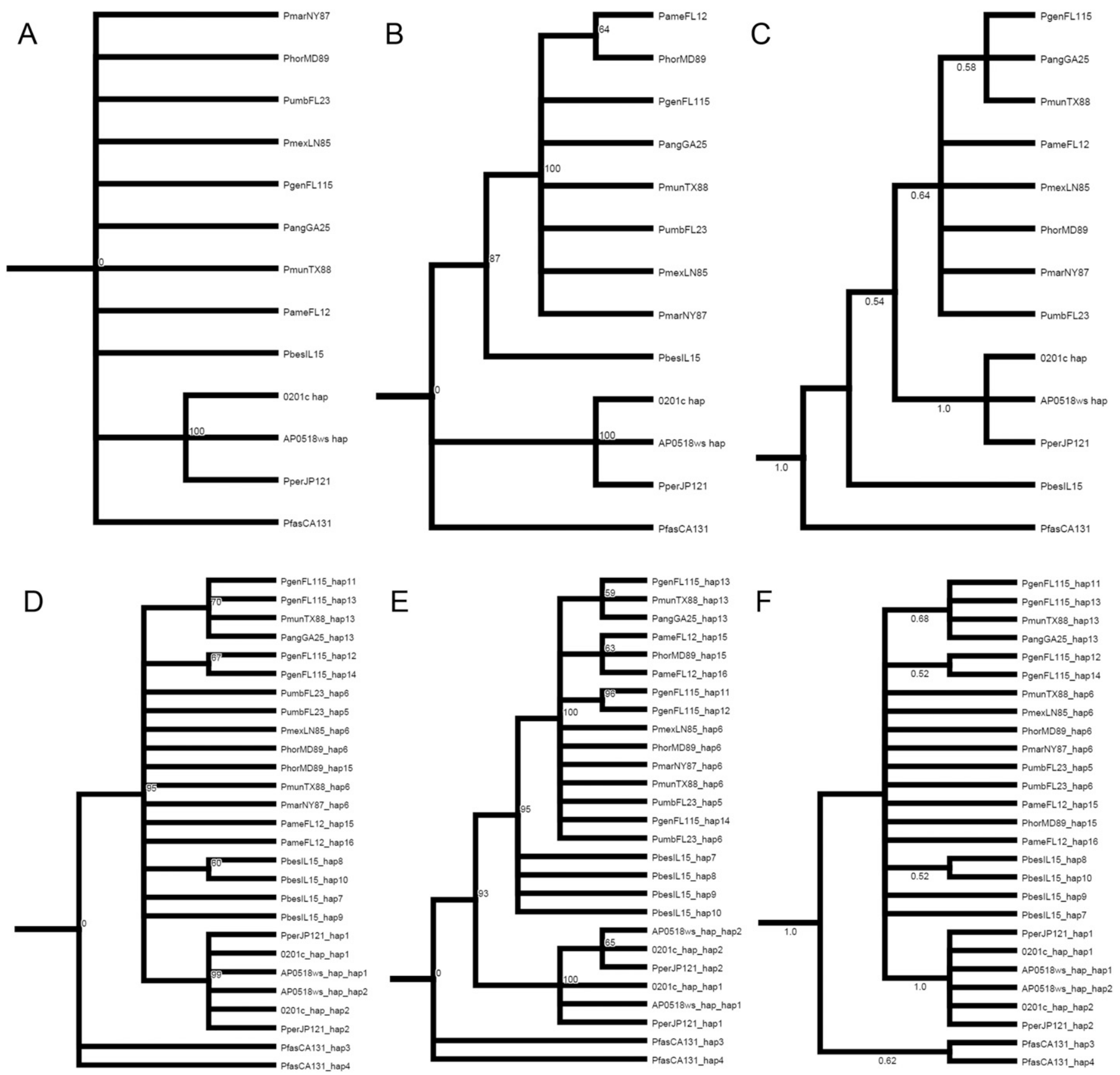

Supplemental Fig. 59. Majority rule consensus trees for TFL1-ATC diploid sequence data using (A) maximum parsimony, (B) maximum parsimony (including gaps), and (C) maximum likelihood; and haplotypic sequence data using (D) maximum parsimony, (E) maximum parsimony (including gaps), and (F) maximum likelihood. Bootstrap values greater than $50 \%$ are described above the branches for maximum parsimony tree. Posterior probability values are described above the branches for maximum likehood tree. Trees are rooted with Prunus fasciculata (PfasCA131). Other species in the cladogram are Prunus americana (PameFL12), Prunus angustifolia (PangGA25), Prunus geniculata (PgenFL115), Prunus hortulana (PhorMD89), Prunus maritima (PmarNY87), Prunus mexicana (PmexLN85), Prunus munsoniana (PmunTX88), Prunus persica (PperJP121, 0201c_hap, AP0518ws_hap), Prunus pumila (PbesIL15), and Prunus umbellata (PumbFL23). 

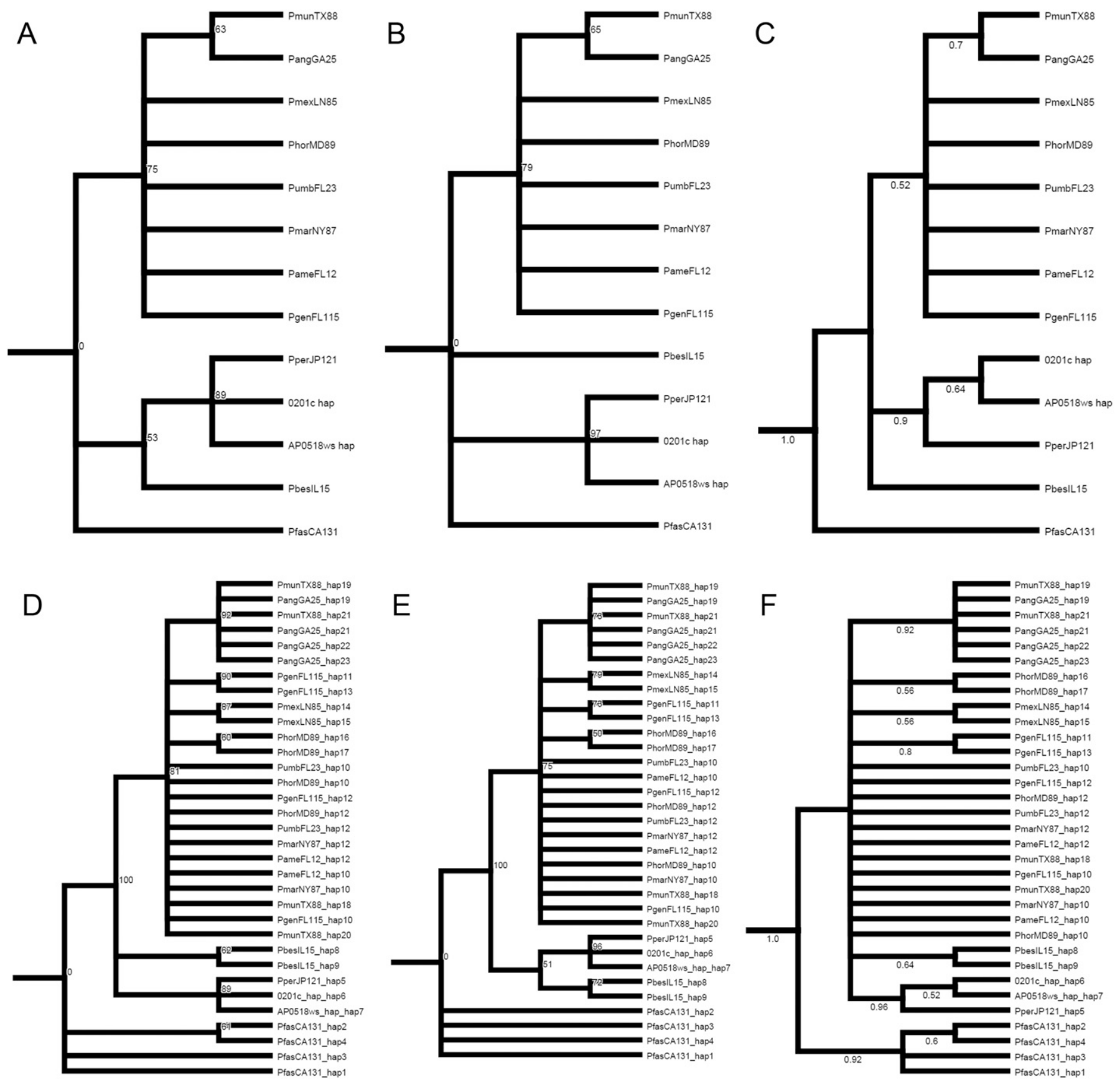

Supplemental Fig. 60. Majority rule consensus trees for TFL2 diploid sequence data using (A) maximum parsimony, (B) maximum parsimony (including gaps), and (C) maximum likelihood; and haplotypic sequence data using (D) maximum parsimony, (E) maximum parsimony (including gaps), and (F) maximum likelihood. Bootstrap values greater than $50 \%$ are described above the branches for maximum parsimony tree. Posterior probability values are described above the branches for maximum likehood tree. Trees are rooted with Prunus fasciculata (PfasCA131). Other species in the cladogram are Prunus americana (PameFL12), Prunus angustifolia (PangGA25), Prunus geniculata (PgenFL115), Prunus hortulana (PhorMD89), Prunus maritima (PmarNY87), Prunus mexicana (PmexLN85), Prunus munsoniana (PmunTX88), Prunus persica (PperJP121, 0201c_hap, AP0518ws_hap), Prunus pumila (PbesIL15), and Prunus umbellata (PumbFL23). 

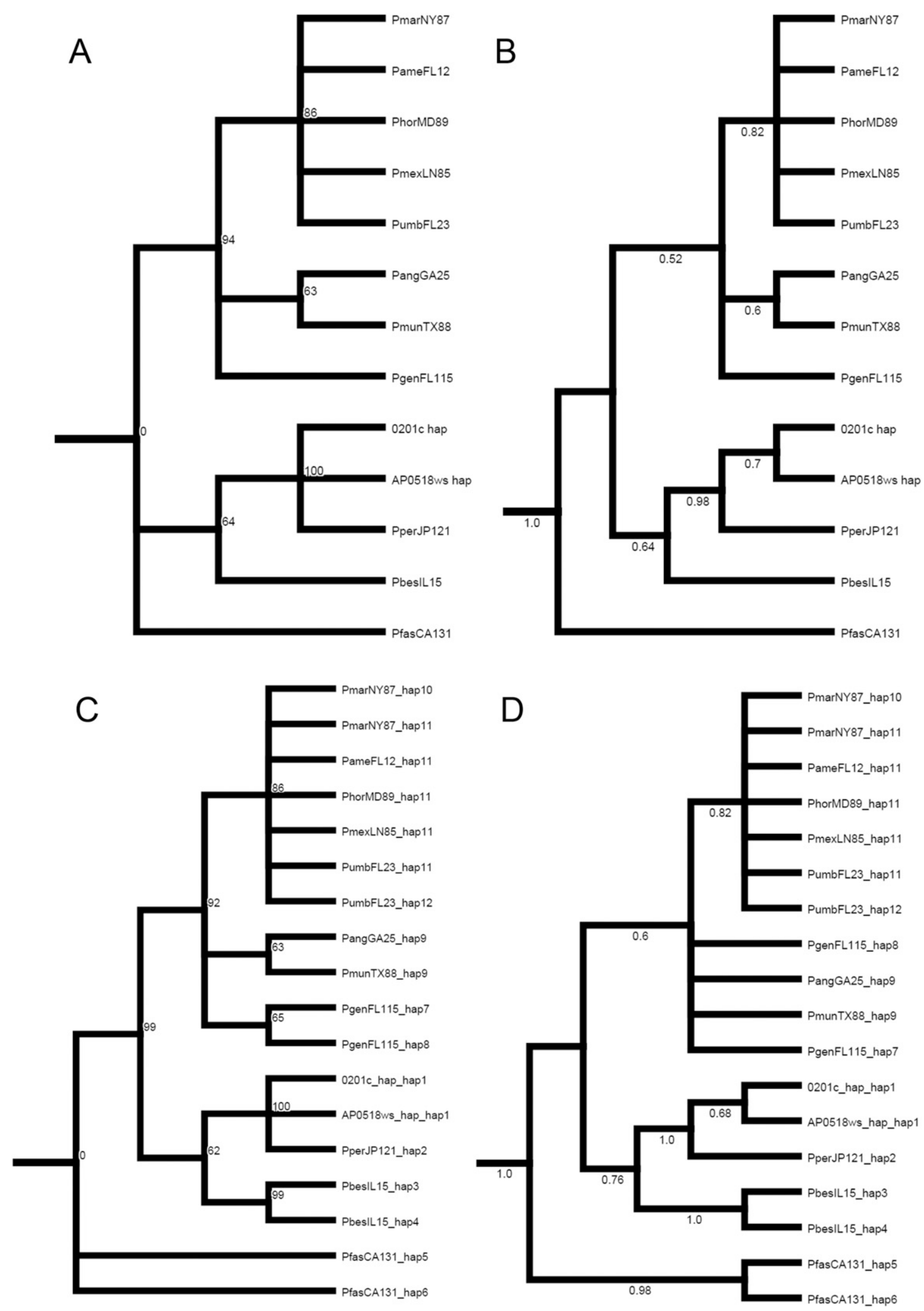

Supplemental Fig. 61. Majority rule consensus trees for VRN1 diploid sequence data using (A) maximum parsimony and (B) maximum likelihood; and haplotypic sequence data using (C) maximum parsimony and (D) maximum likelihood. Bootstrap values greater than 50\% are described above the branches for maximum parsimony tree. Posterior probability values are described above the branches for maximum likehood tree. Trees are rooted with Prunus fasciculata (PfasCA131). Other species in the cladogram are Prunus americana (PameFL12), Prunus angustifolia (PangGA25), Prunus geniculata (PgenFL115), Prunus hortulana (PhorMD89), Prunus maritima (PmarNY87), Prunus mexicana (PmexLN85), Prunus munsoniana (PmunTX88), Prunus persica (PperJP121, 0201c_hap, AP0518ws hap), Prunus pumila (PbesIL15), and Prunus umbellata (PumbFL23). 

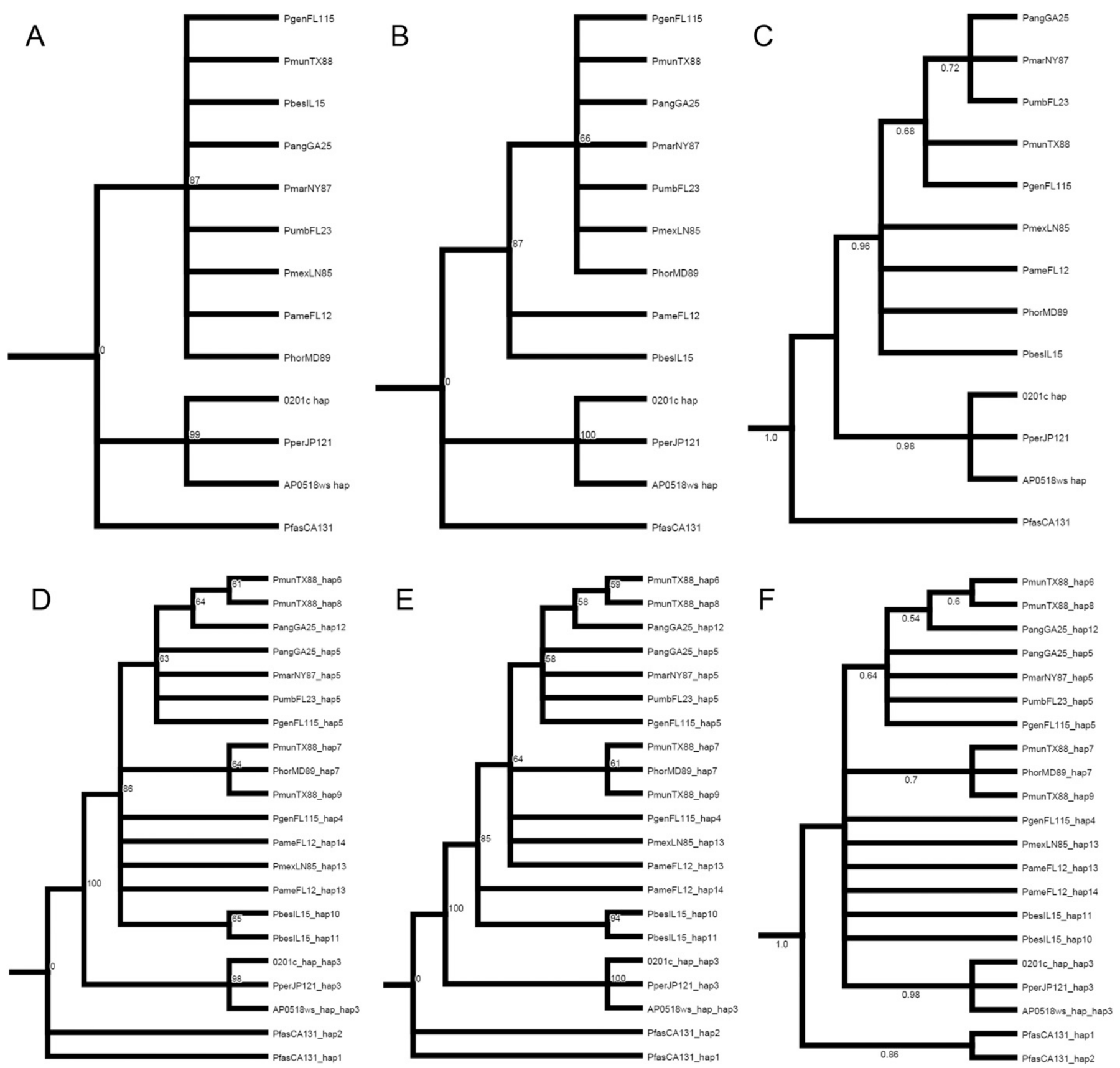

Supplemental Fig. 62. Majority rule consensus trees for VRN2 diploid sequence data using (A) maximum parsimony, (B) maximum parsimony (including gaps), and (C) maximum likelihood; and haplotypic sequence data using (D) maximum parsimony, (E) maximum parsimony (including gaps), and (F) maximum likelihood. Bootstrap values greater than $50 \%$ are described above the branches for maximum parsimony tree. Posterior probability values are described above the branches for maximum likehood tree. Trees are rooted with Prunus fasciculata (PfasCA131). Other species in the cladogram are Prunus americana (PameFL12), Prunus angustifolia (PangGA25), Prunus geniculata (PgenFL115), Prunus hortulana (PhorMD89), Prunus maritima (PmarNY87), Prunus mexicana (PmexLN85), Prunus munsoniana (PmunTX88), Prunus persica (PperJP121, 0201c_hap, AP0518ws_hap), Prunus pumila (PbesIL15), and Prunus umbellata (PumbFL23). 

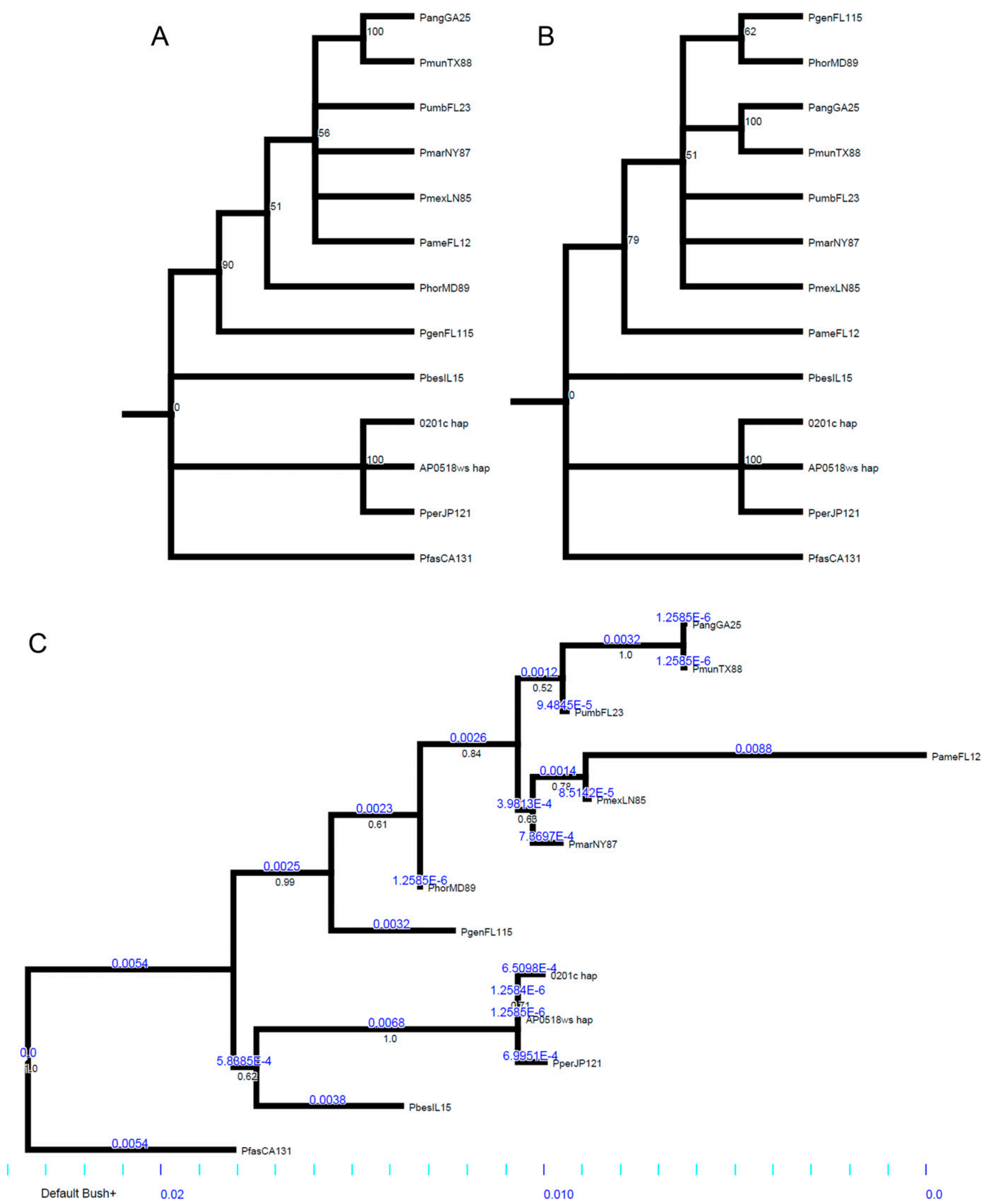

Supplemental Fig. 63. Phylogenetic analyses of combined isozymes diploid sequence data (1399 bp). Majority rule consensus trees using (A) maximum parsimony [38 trees, 82 steps, consistency index $(\mathrm{CI})=0.902$, retention index $(\mathrm{RI})=0.922$, rescaled consistency index $(\mathrm{RC})=0.832]$ and $(\mathbf{B})$ maximum parsimony (including gaps) ( 4 trees, 127 steps, $\mathrm{CI}=0.874, \mathrm{RI}=0.890, \mathrm{RC}=0.778$ ) analyses. Bootstrap values greater than $50 \%$ are described above the branches. $(\mathbf{C})$ Maximum likelihood tree $(-\operatorname{lnL}=2464.46)$. Branch lengths described above the branches and posterior probability values below the branches. Trees are rooted with Prunus fasciculata (PfasCA131). Other species in the cladogram are Prunus americana (PameFL12), Prunus angustifolia (PangGA25), Prunus geniculata (PgenFL115), Prunus hortulana (PhorMD89), Prunus maritima (PmarNY87), Prunus mexicana (PmexLN85), Prunus munsoniana (PmunTX88), Prunus persica (PperJP121, 0201c_hap, AP0518ws_hap), Prunus pumila (PbesIL15), and Prunus umbellata (PumbFL23). 
A

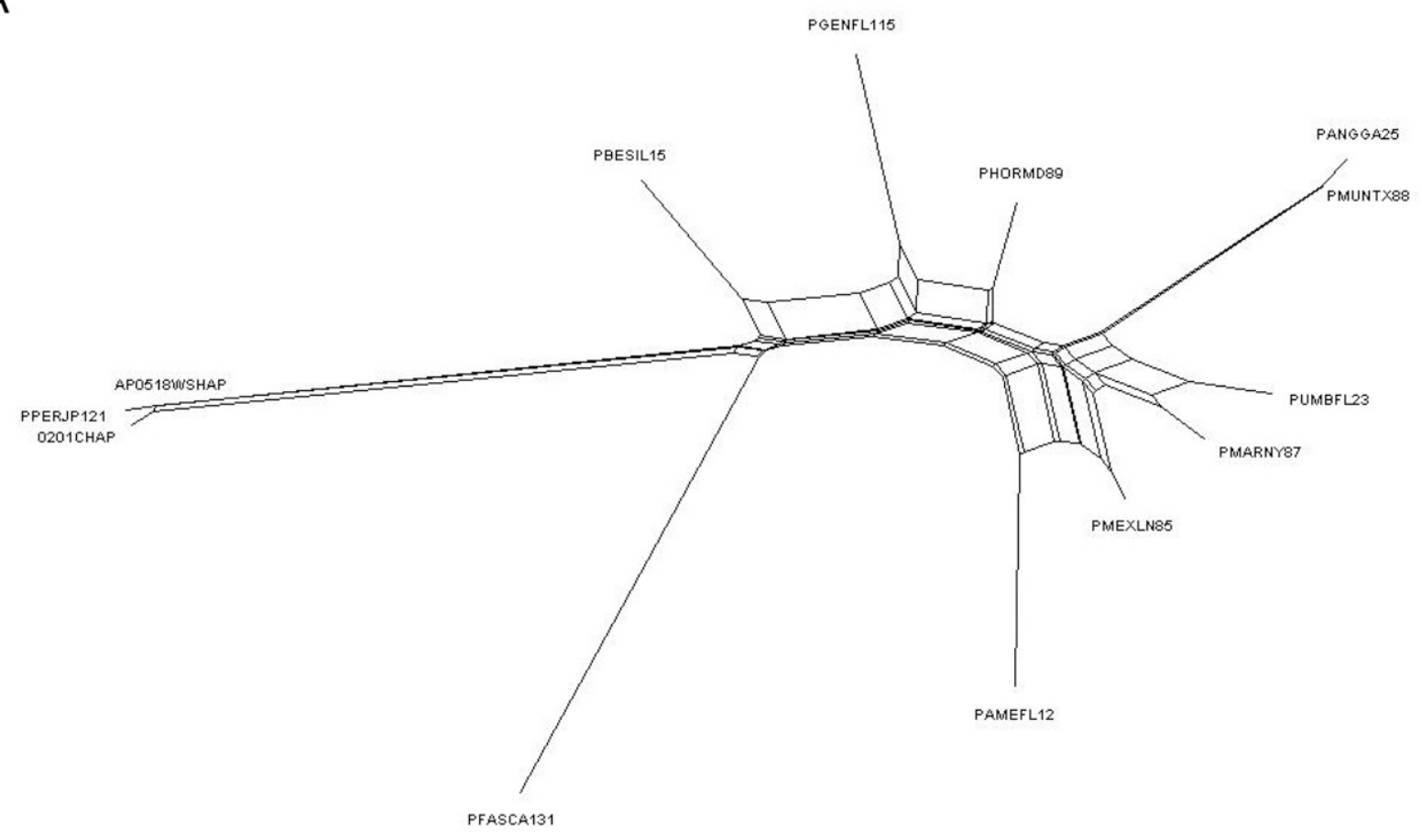

B

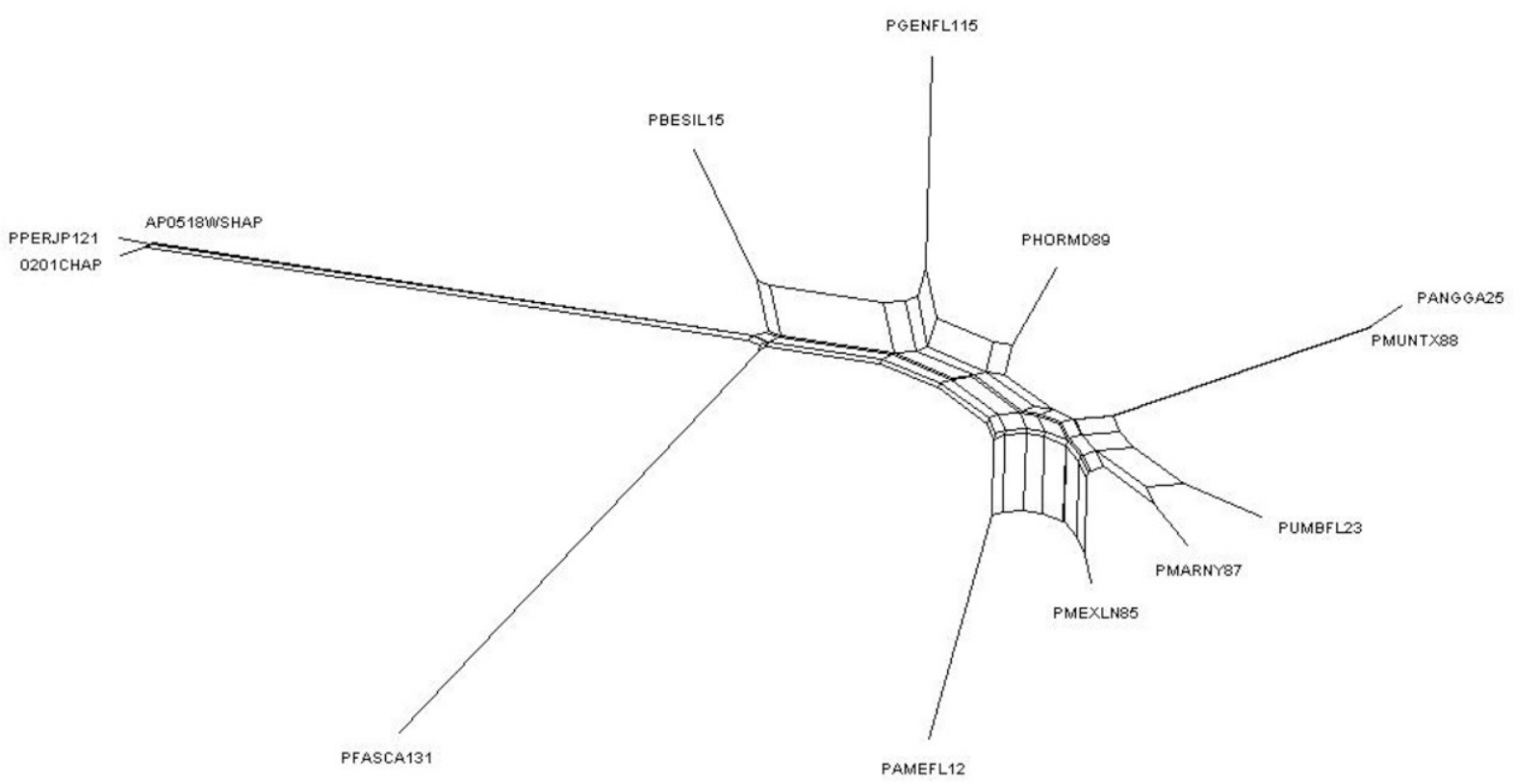

Supplemental Fig. 64. A nonstandarized multilocus combined isozymes genes sequence data network using (A) gaps as missing characters and (B) gaps as fifth characters. Prunus species in the cladogram are Prunus americana (PameFL12), Prunus angustifolia (PangGA25), Prunus fasciculata (PfasCA131), Prunus geniculata (PgenFL115), Prunus hortulana (PhorMD89), Prunus maritima (PmarNY87), Prunus mexicana (PmexLN85), Prunus munsoniana (PmunTX88), Prunus persica (PperJP121, 0201c_hap, AP0518ws_hap), Prunus pumila (PbesIL15), and Prunus umbellata (PumbFL23). 

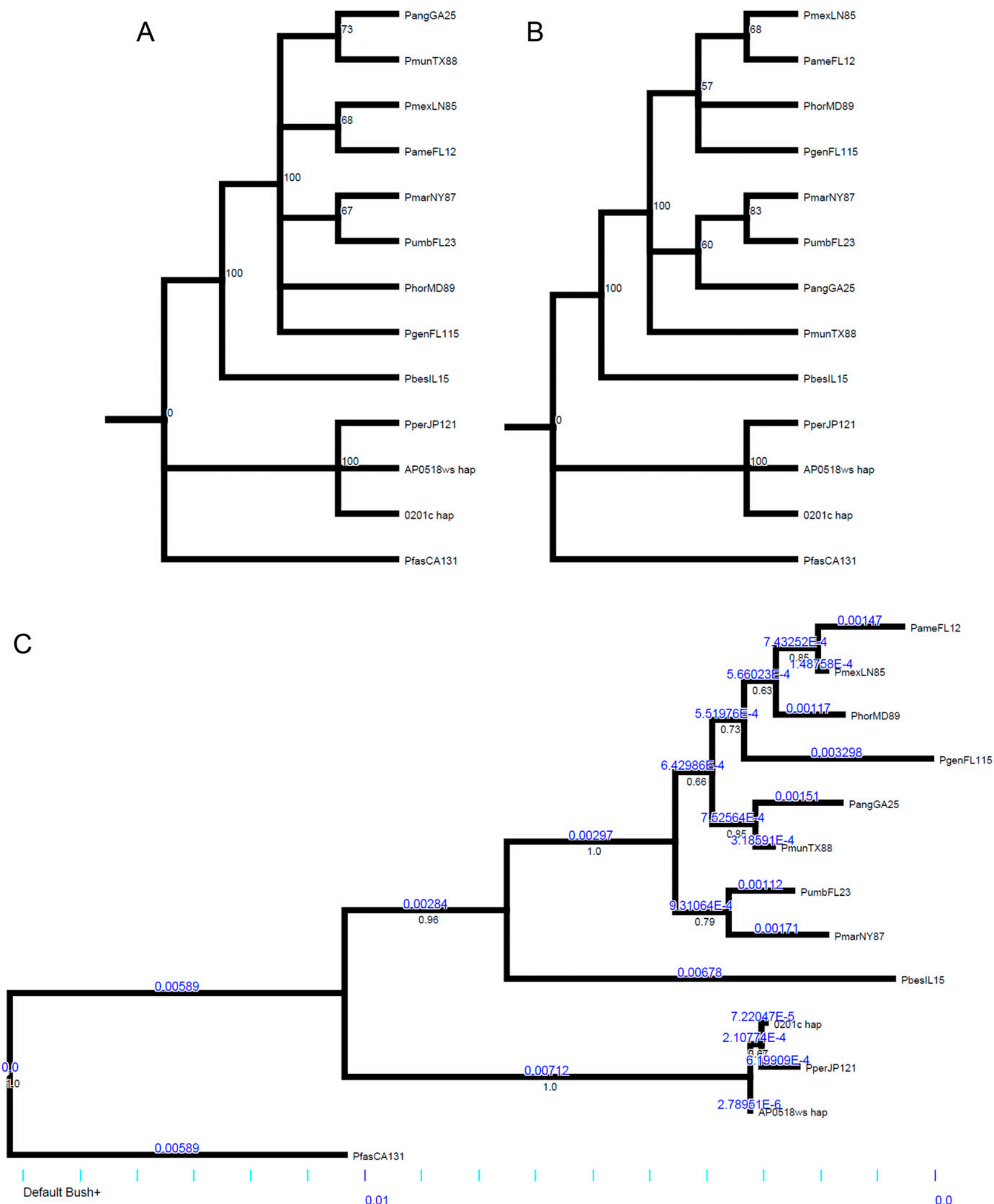

0.01

0.0

Supplemental Fig. 65. Phylogenetic analyses of combined tree architecture genes diploid sequence data (6659 bp). Majority rule consensus trees using (A) maximum parsimony [10 trees, 363 steps, consistency index $(\mathrm{CI})=0.917$, retention index $(\mathrm{RI})=0.927$, rescaled consistency index $(\mathrm{RC})=0.850]$ and $(\mathbf{B})$ maximum parsimony (including gaps) $(2$ trees, 560 steps, $\mathrm{CI}=0.914, \mathrm{RI}=0.920, \mathrm{RC}=0.842)$ analyses. Bootstrap values greater than $50 \%$ are described above the branches. (C) Maximum likelihood tree $(-\operatorname{lnL}=11,668.52)$. Branch lengths described above the branches and posterior probability values below the branches. Trees are rooted with Prunus fasciculata (PfasCA131). Other species in the cladogram are Prunus americana (PameFL12), Prunus angustifolia (PangGA25), Prunus geniculata (PgenFL115), Prunus hortulana (PhorMD89), Prunus maritima (PmarNY87), Prunus mexicana (PmexLN85), Prunus munsoniana (PmunTX88), Prunus persica (PperJP121, 0201c_hap, AP0518ws_hap), Prunus pumila (PbesIL15), and Prunus umbellata (PumbFL23). 
A

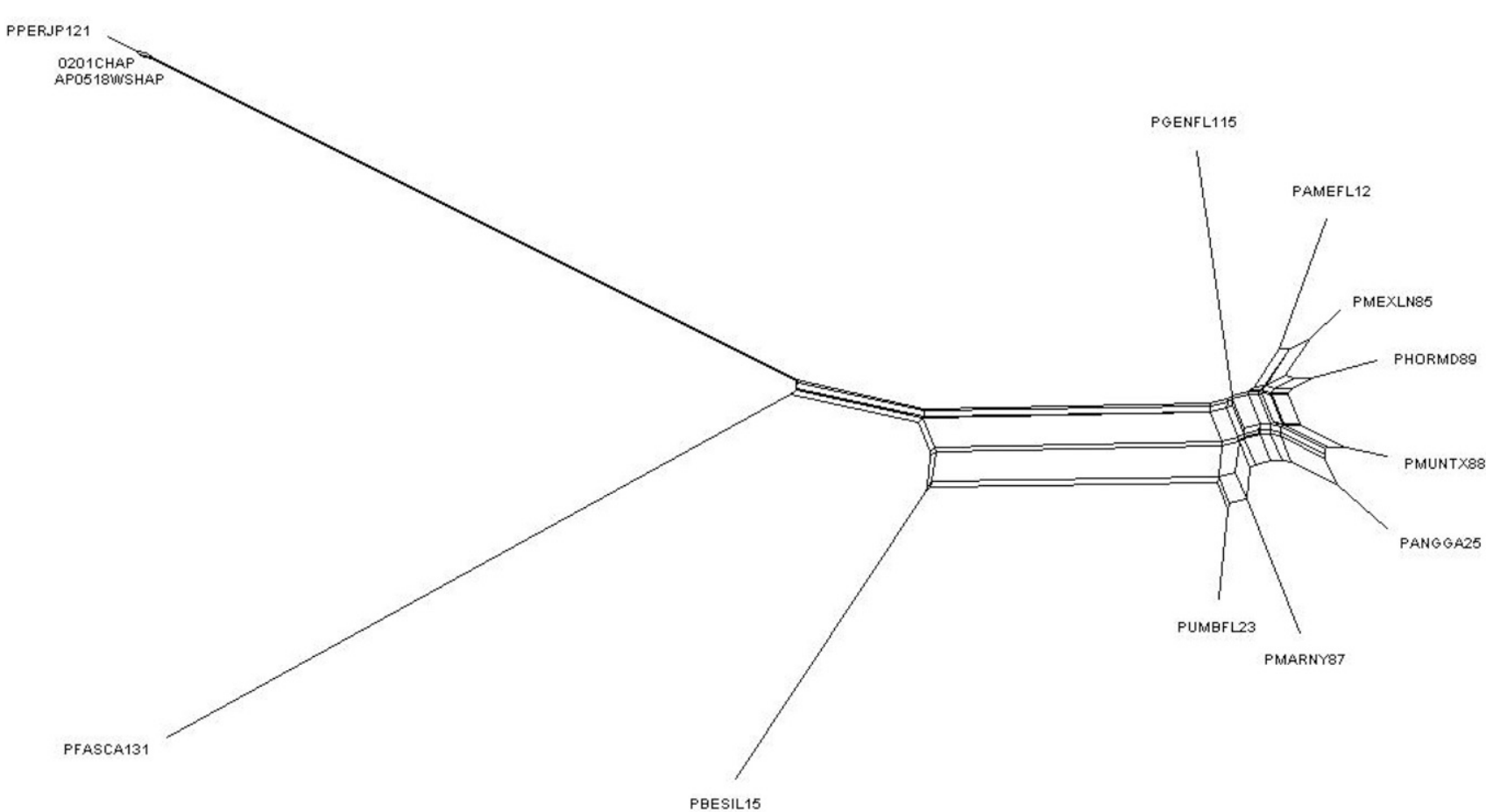

B

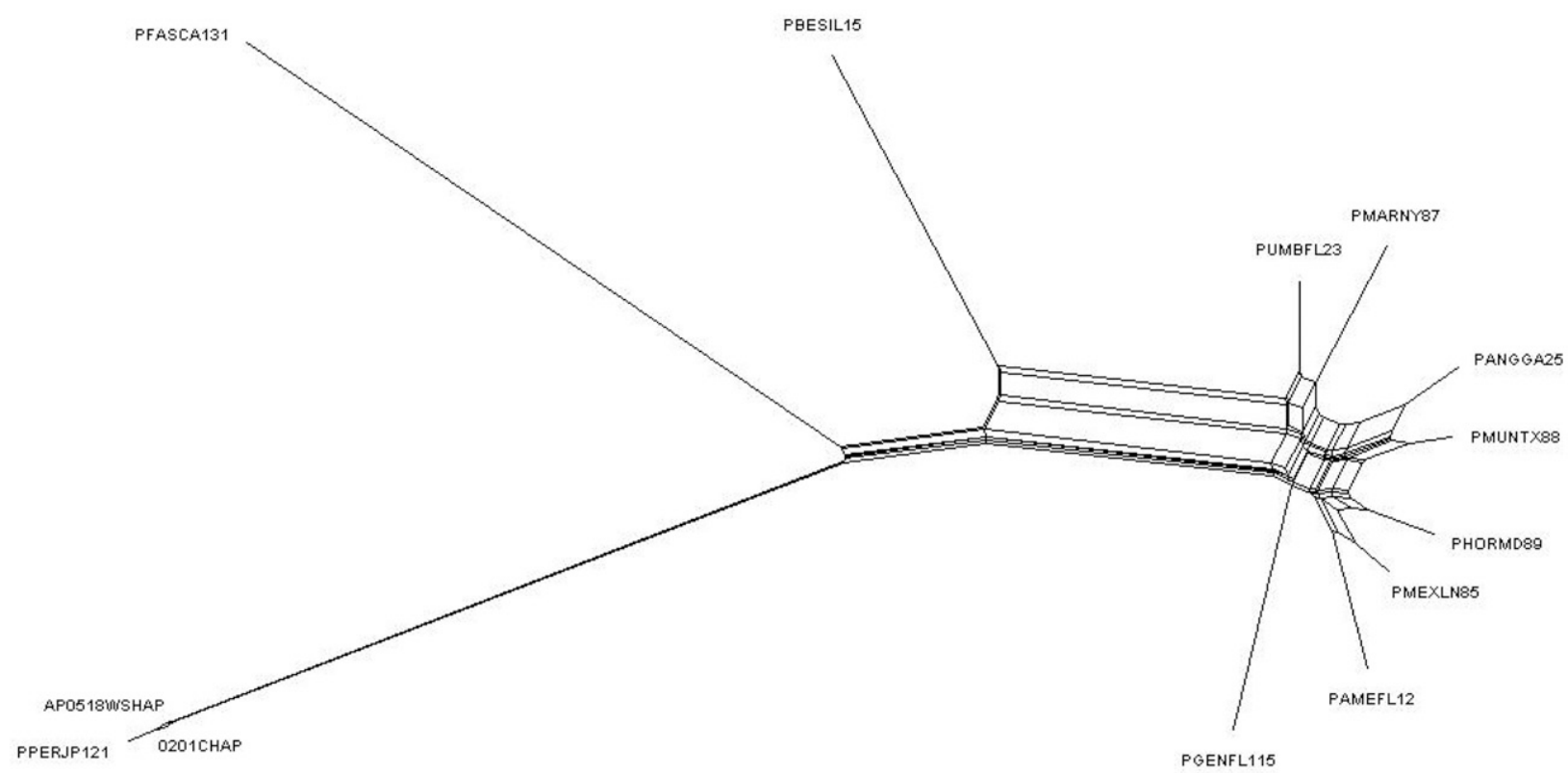

Supplemental Fig. 66. A nonstandarized multilocus combined tree architecture genes sequence data network using (A) gaps as missing characters and (B) gaps as fifth characters. Prunus species in the cladogram are Prunus americana (PameFL12), Prunus angustifolia (PangGA25), Prunus fasciculata (PfasCA131), Prunus geniculata (PgenFL115), Prunus hortulana (PhorMD89), Prunus maritima (PmarNY87), Prunus mexicana (PmexLN85), Prunus munsoniana (PmunTX88), Prunus persica (PperJP121, 0201c_hap, AP0518ws_hap), Prunus pumila (PbesIL15), and Prunus umbellata (PumbFL23). 

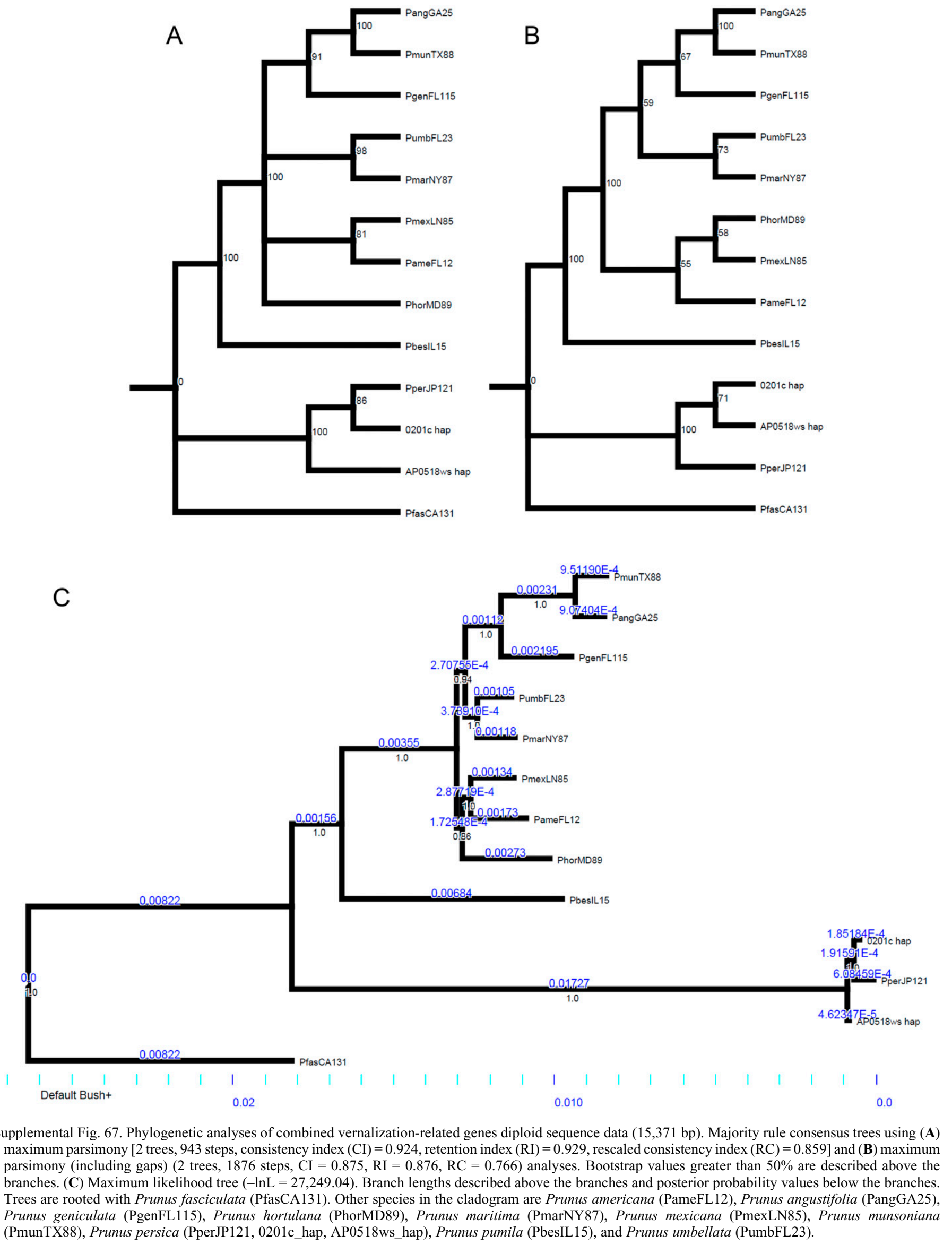

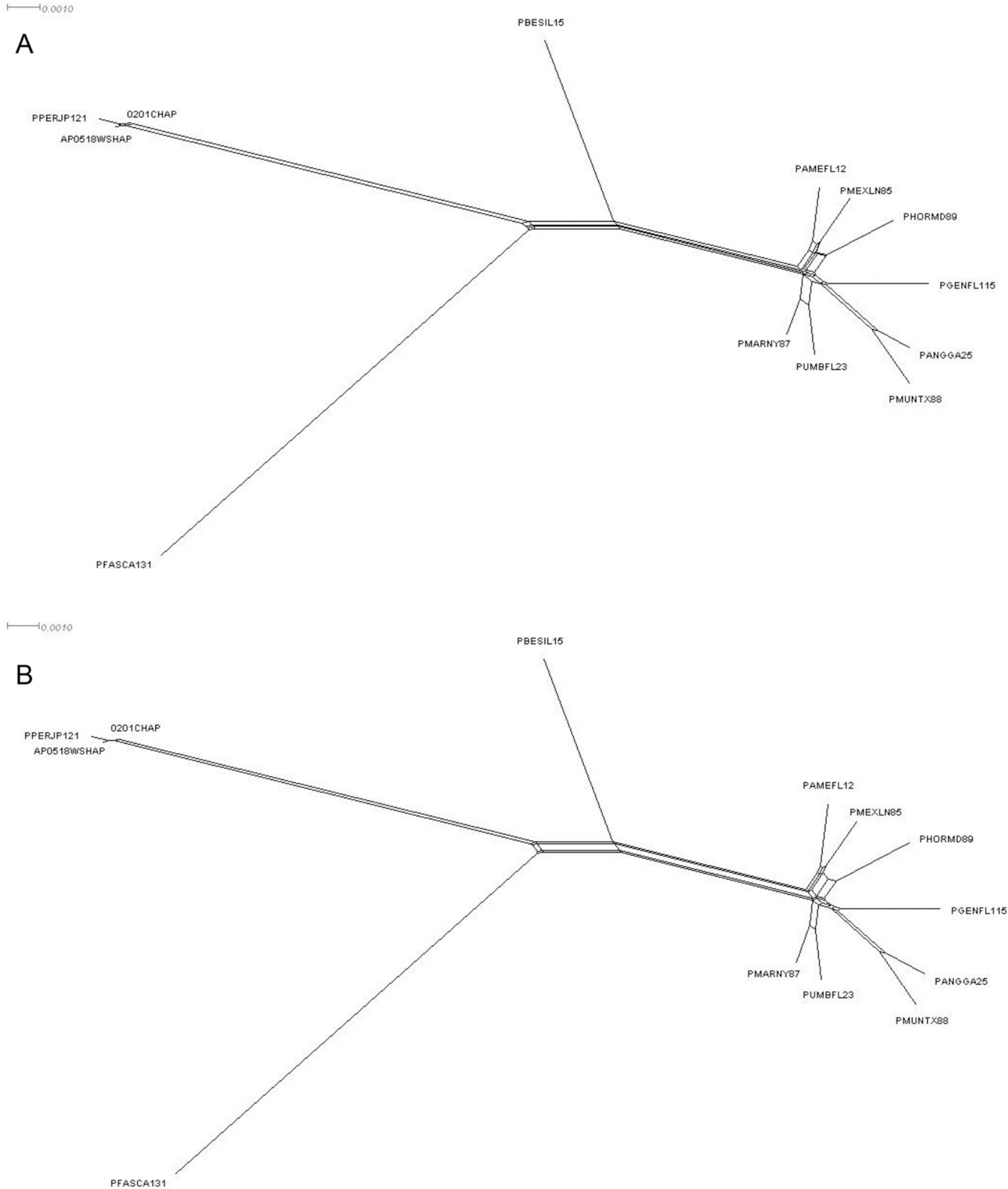

Supplemental Fig. 68. A nonstandarized multilocus combined vernalization-related genes sequence data network using (A) gaps as missing characters and (B) gaps as fifth characters. Prunus species in the cladogram are Prunus americana (PameFL12), Prunus angustifolia (PangGA25), Prunus fasciculata (PfasCA131), Prunus geniculata (PgenFL115), Prunus hortulana (PhorMD89), Prunus maritima (PmarNY87), Prunus mexicana (PmexLN85), Prunus munsoniana (PmunTX88), Prunus persica (PperJP121, 0201c_hap, AP0518ws_hap), Prunus pumila (PbesIL15), and Prunus umbellata (PumbFL23). 
Supplemental Table 1. Simple sequence repeat markers selected from the 'Texas' almond (Prunus dulcis) $\times$ 'Earlygold' peach $(\mathrm{T} \times \mathrm{E})$ reference map.

\begin{tabular}{|c|c|c|c|c|c|c|}
\hline Chromosome & Position & Marker & Fluorophore & $\mathrm{Ta}\left({ }^{\circ} \mathrm{C}\right)^{\mathrm{z}}$ & Forward sequence & Reverse sequence \\
\hline 1 & 9 & CPSCT008 & HEX & 62 & TGGATCCAATCCAAGAGTCTG & GCAGCAAGTTGTTCTTGGTTC \\
\hline 1 & 25.8 & СPDCT038 & HEX & 62 & ATCACAGGTGAAGGCTGTGG & CAGATTCATTGGCCCATCTT \\
\hline 1 & 33.9 & СРРСТ026 & HEX & 55 & AGACGCAGCACCCAAACTAC & CATTACATCACCGCCAACAA \\
\hline 1 & 47.3 & ВРРСТ027 & HEX & 57 & GGACGGACAGAAATGAAGGT & CCTTAACCCACGCAACTCC \\
\hline 1 & 55.2 & ВРРСТ016 & HEX & 57 & GATTGAGAGATTGGGCTGC & GAGGATTCTCATGATTTGTGC \\
\hline 1 & 65.1 & СРРСТ029 & HEX & 55 & ССАААТТССАААТСТССТААСА & TGATCAACTTTGAGATTTGTTGAA \\
\hline 1 & 77.4 & ВРРСТ028 & HEX & 57 & TCAAGTTAGCTGAGGATCGC & GAGCTTGCCTATGAGAAGACC \\
\hline 2 & 9.6 & UDP98-025 & FAM & 57 & GGGAGGTTACTATGCCATGAAG & CGCAGACATGTAGTAGGACCTC \\
\hline 2 & 25 & ВРРСТ013 & HEX & 57 & ACCCACAAATCAAGCATATCC & AGCTTCAGCCACCAAGC \\
\hline 2 & 38 & ВРРСТ030 & HEX & 57 & AATTGTACTTGCCAATGCTATGA & CTGCCTTCTGCTCACACC \\
\hline 2 & 48.6 & CPSCT034 & HEX & 62 & AGGTGGACAATAGCCGTGAT & TTTCCAGACCCTGAGAAAGC \\
\hline 3 & 18 & ВРРСТ039 & FAM & 57 & ATTACGTACCCTAAAGCTTCTGC & GATGTCATGAAGATTGGAGAGG \\
\hline 3 & 36.4 & СPDCT025 & HEX & 62 & GACCTCATCAGCATCACCAA & TTCCCTAACGTCCCTGACAC \\
\hline 3 & 46.4 & СРDCT027 & HEX & 62 & TGAGGAGAGCACTGGAGGAG & CAACCGATCCCTCTAGACCA \\
\hline 4 & 10.4 & СРРСТ005 & FAM & 52 & CATGAACTCTACTCTCCA & TGGTATGGACTCACCAAC \\
\hline 4 & 28.3 & UDP96-003 & FAM & 57 & TTGCTCAAAAGTGTCGTTGC & ACACGTAGTGCAACACTGGC \\
\hline 4 & 34.1 & EPDC3832 & FAM & 57 & CTTTTGAAGGCCCAATACCA & ATCACTGCTTCGCCTTCATT \\
\hline 4 & 45.4 & ВРРСТ023 & HEX & 57 & TGCAGCTCATTACCTTTTGC & AGATGTGCTCGTAGTTCGGAC \\
\hline 4 & 52.7 & EPPISF032 & HEX & 57 & TCCCCCACAGATATTTCAGC & GTCGAGGAGAGAGGGCTTTT \\
\hline 5 & 5.2 & ВРРСТ026 & FAM & 57 & ATACCTTTGCCACTTGCG & TGAGTTGGAAGAAAACGTAACA \\
\hline 5 & 20.1 & ВРРСТ017 & HEX & 57 & TTAAGAGTTTGTGATGGGAACC & AAGCATAATTTAGCATAACCAAGC \\
\hline 5 & 32.9 & ВРРСТ038 & FAM & 57 & TATATTGTTGGCTTCTTGCATG & GAGCTTGCCTATGAGAAGACC \\
\hline 5 & 44 & ВРРСТ014 & HEX & 57 & TTGTCTGCCTCTCATCTTAACC & CATCGCAGAGAACTGAGAGC \\
\hline 6 & 8.7 & СРРСТ008 & FAM & 59 & GAGCTCTCACGCATTAGTTT & TTTGACTGCATAACAAAACG \\
\hline 6 & 17.5 & UDP96-001 & HEX & 57 & AGTTTGATTTTCTGATGCATCC & TGCCATAAGGACCGGTATGT \\
\hline 6 & 30.1 & ВРРСТ008 & FAM & 57 & ATGGTGTGTATGGACATGATGA & ССТСАAССТАAGACACСТTCACT \\
\hline 6 & 35.8 & СРРСТ015 & FAM & 50 & TGGAGTGCCAATACTATTTA & CATATGCATGGTTATGGT \\
\hline 6 & 41 & EPPISF002 & FAM & 56 & CGACGTGTGACCAAAGGAC & GCAАCТССАТССАСАТТТСТС \\
\hline 6 & 56.4 & ВРРСТ025 & FAM & 57 & TCCTGCGTAGAAGAAGGTAGC & CGACATAAAGTCCAAATGGC \\
\hline 6 & 72 & UDP98-412 & HEX & 57 & AGGGAAAGTTTCTGCTGCAC & GCTGAAGACGACGATGATGA \\
\hline 7 & 9.5 & CPSCT004 & FAM & 62 & GCTCTGAAGCTCTGCATTGA & TTTGAAATGGCTATGGAGTACG \\
\hline 7 & 18.6 & СРРСТ022 & FAM & 50 & CAATTAGCTAGAGAGAATTATTG & GACAAGAAGCAAGTAGTTTG \\
\hline 7 & 29.6 & ВРРСТ029 & FAM & 57 & GGACGGACAGAAATGAAGGT & СCTTAACCCACGCAACTCC \\
\hline 7 & 38.9 & СРРСТ033 & HEX & 50 & TCAGCAAACTAGAAACAAACC & TTGCAATCTGGTTGATGTT \\
\hline 7 & 47.8 & PMS2 & FAM & 55 & CACTGTCTCCCAGGTTAAACT & CCTGAGCTTTTGACACATGC \\
\hline 7 & 64.7 & EPDCU3392 & HEX & 57 & СTTTTCATGGGTTCCTCACC & ATCAACCAGTTCACGCACAA \\
\hline 8 & 14.1 & ВРРСТ006 & HEX & 57 & GCTTGTGGCATGGAAGC & СССТGTTTCTCATAGAACTCACAT \\
\hline 8 & 20.8 & UDP96-019 & HEX & 57 & TTGGTCATGAGCTAAGAAAACA & TAGTGGCACAGAGCAACACC \\
\hline 8 & 24.8 & СРРСТ006 & HEX & 59 & AATTAACTCCAACAGCTCCA & ATGGTTGCTTAATTCAATGG \\
\hline 8 & 42.6 & СPDCT023 & FAM & 62 & GTGGCAAATGTTGGCAAAG & AACACAAAGCAGCACCAAGA \\
\hline 8 & 54.7 & EPDCU3117 & FAM & 57 & CAGAGGGAACAGTGTGAGCA & TGTTGTTGTCGACCCTGAAA \\
\hline
\end{tabular}

${ }^{\mathrm{z}} \mathrm{Ta}=$ annealing temperature. 
Supplemental Table 2. Primer sequences of chloroplast DNA as described by Shaw et al. (2005, 2007) and Morris et al. (2008).

\begin{tabular}{|c|c|}
\hline Region & Primer name and sequence $\left(5^{\prime}-3^{\prime}\right)$ \\
\hline \multirow[t]{2}{*}{$3^{\prime} t r n V-n d h C$} & $\operatorname{trnV}^{(\mathrm{UAC})} \mathrm{x} 2:$ GTC TAC GGT TCG ART CCG TA \\
\hline & ndhC: TAT TAT TAG AAA TGY CCA RAA AAT ATC ATA TTC \\
\hline$n d h J-t r n F$ & ndhJ: ATG CCY GAA AGT TGG ATA GG \\
\hline \multirow[t]{2}{*}{$\operatorname{trn} L$-trnF and $\operatorname{trn} L$ intron } & $5^{\prime} \operatorname{trnL}^{(\mathrm{UAA})}-\mathrm{F}:$ CGA AAT CGG TAG ACG CTA CG \\
\hline & $\operatorname{trnF}{ }^{(\mathrm{GAA})}$ : ATT TGA ACT GGT GAC ACG AG (Taberlet et al., 1991) \\
\hline $\operatorname{trn} T$-trnL & $\operatorname{trnT}^{(\mathrm{UGU})}-\mathrm{F}: \mathrm{CAT}$ TAC AAA TGC GAT GCT CT \\
\hline \multirow[t]{2}{*}{$\operatorname{trn} Q-5^{\prime} r p s 16$} & $\operatorname{trnQ}{ }^{(U U G)}:$ GCG TGG CCA AGY GGT AAG GC \\
\hline & rpS16x1: GTT GCT TTY TAC CAC ATC GTT T \\
\hline $\operatorname{trnH}-p s b A$ & $\begin{array}{l}\text { trnH }{ }^{(G U G)} \text { : CGC GCA TGG TGG ATT CAC AAT CC (Tate and Simpson, 2003) } \\
\text { psbA: GTT ATG CAT GAA CGT AAT GCT C (Sang et al., 1997) }\end{array}$ \\
\hline \multirow[t]{2}{*}{$n d h F-r p l 32$} & rpL32-R: CCA ATA TCC CTT YYT TTT CCA A \\
\hline & ndhF: GAA AGG TAT KAT CCA YGM ATA TT \\
\hline
\end{tabular}




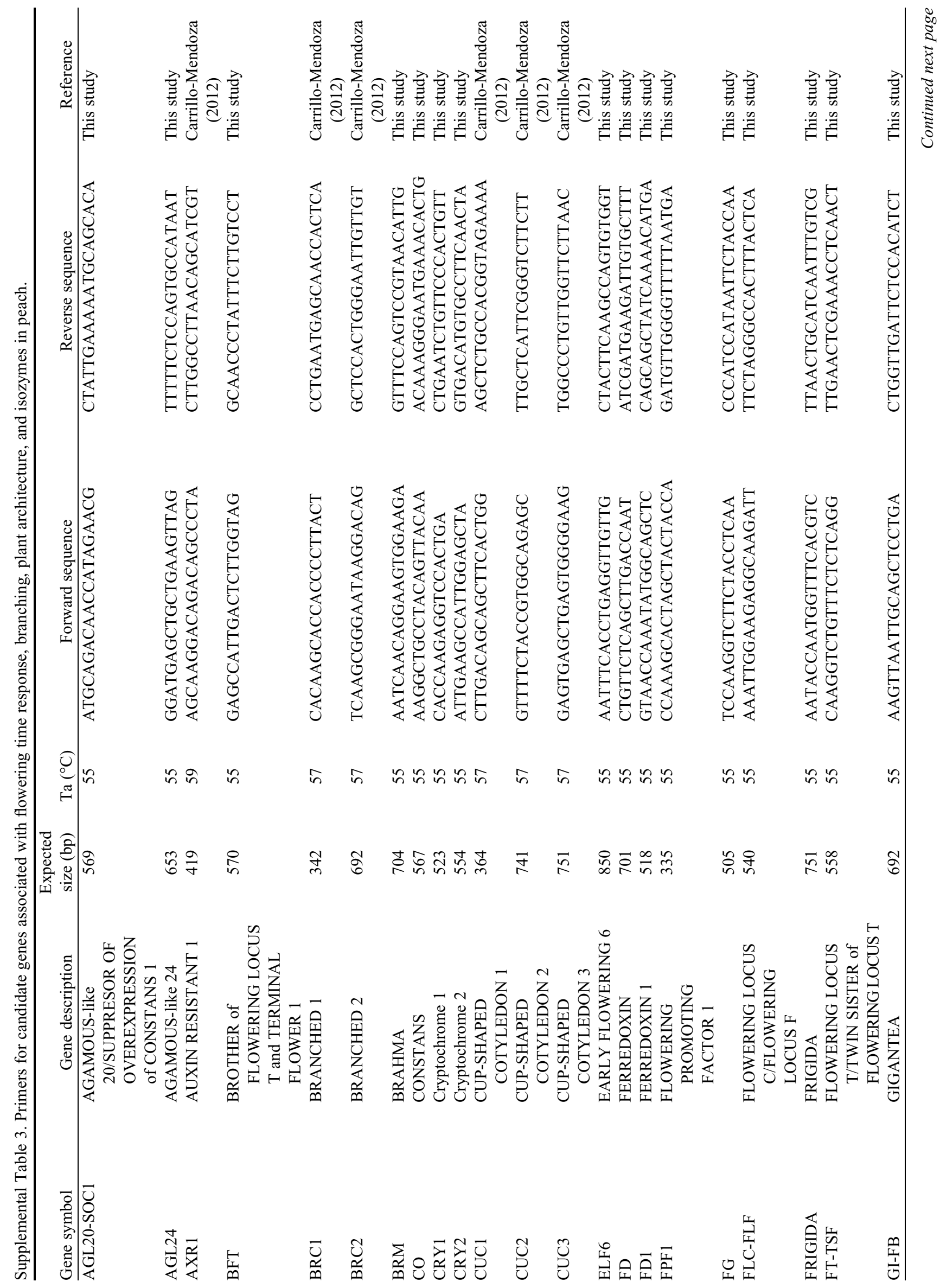




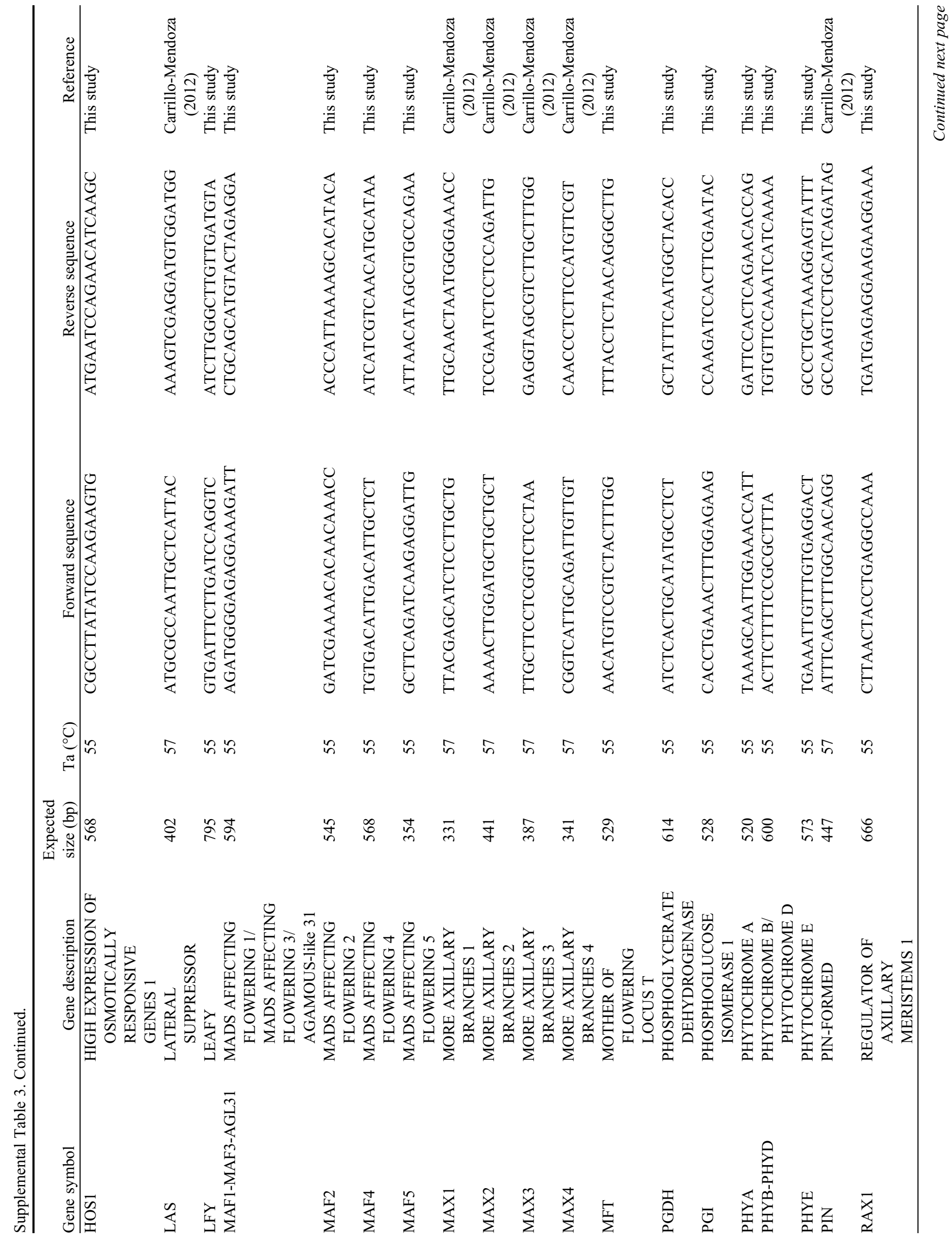




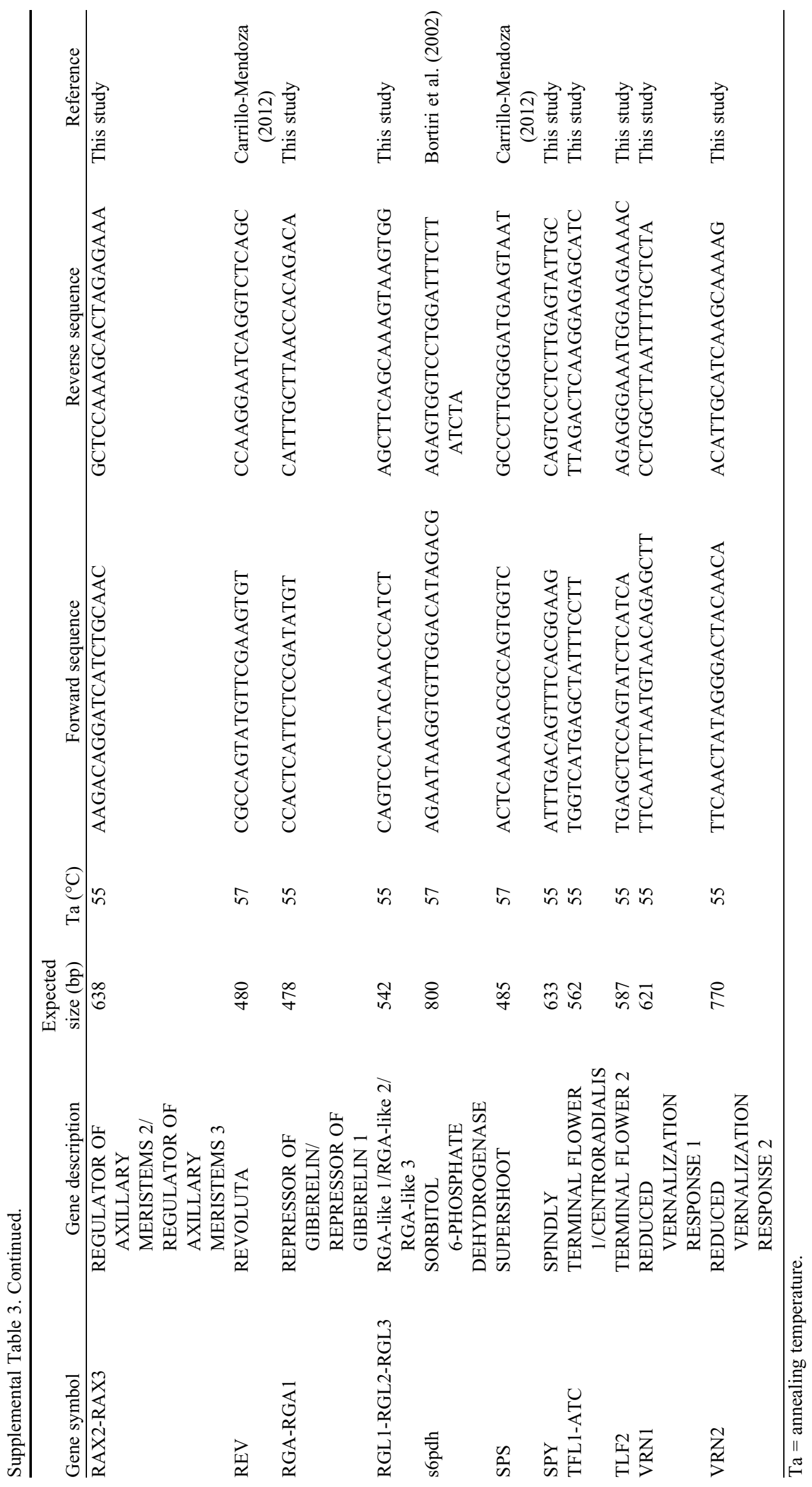


Supplemental Table 4. Internal primers used to improve sequence coverage, quality, and base-calling accuracy of gene regions associated with axillary meristem formation, vernalization response, and isozyme expression.

\begin{tabular}{|c|c|c|}
\hline Gene symbol & $\mathrm{Ta}\left({ }^{\circ} \mathrm{C}\right)$ & Sequence \\
\hline CUC2int-F & 55 & GAACTTTAGTGATCTCATGCTA \\
\hline CUC3int-R & 55 & CAAGCTGAAGAAGTACCACT \\
\hline RAX2-RAX3int-F & 55 & TCAATGTTTGTTCTCTCACA \\
\hline PGIint-R & 55 & CAGAGAACTTTTGGATCTTG \\
\hline PGDHint-F & 55 & CAAAGGTGTTCAATGATGAA \\
\hline PGDHint-R & 55 & GTGTGAGAGTGACATTCTCA \\
\hline AGL20-SOC1 int-F & 55 & TGACCAAAATATGCAGGTGA \\
\hline AGL20-SOC1int-R & 55 & СТCTCCAACTGTTGCTCAAT \\
\hline BRMint-F & 55 & CTGGCCTCTTAATTTGTC \\
\hline COint-F & 55 & CTTTGCTTAATGCTAGCTCA \\
\hline COint-R & 55 & CTGAAGATACTCCAAGAATATT \\
\hline ELF6int-F & 55 & CAATTCCTTTGTGAACAT \\
\hline FT-TSFint-R & 55 & AATACACACCAATGCAAATA \\
\hline GI-FBint-F & 55 & ATGTTAGGTCGTTGATGCAT \\
\hline GI-FBint-R & 55 & TTAGACCTGAAGAACCACTG \\
\hline HOS1 int-F & 55 & GACGTCCAACGCCTTTATTC \\
\hline HOS1 int-R & 55 & CATTAGATATGCCAGCCAAT \\
\hline LFYint-F & 55 & ACCAAGGTACGGAGTTTACC \\
\hline LFYint-R & 55 & TGGAATCACTATATATTTCTAACAA \\
\hline LFYint2-F & 55 & ATATAGTGATTCCAATAATGATG \\
\hline MAF2Aint-R & 55 & TCTCAACTCAACTGAGTACCAT \\
\hline MAF4int-F & 55 & TGATCATGTGTGGGAACTT \\
\hline MAF4int-R & 55 & AAGAAGTTCCCACACATGAT \\
\hline
\end{tabular}

$\mathrm{F}=$ forward sequence; $\mathrm{R}=$ reverse sequence; $\mathrm{Ta}=$ annealing temperature. 


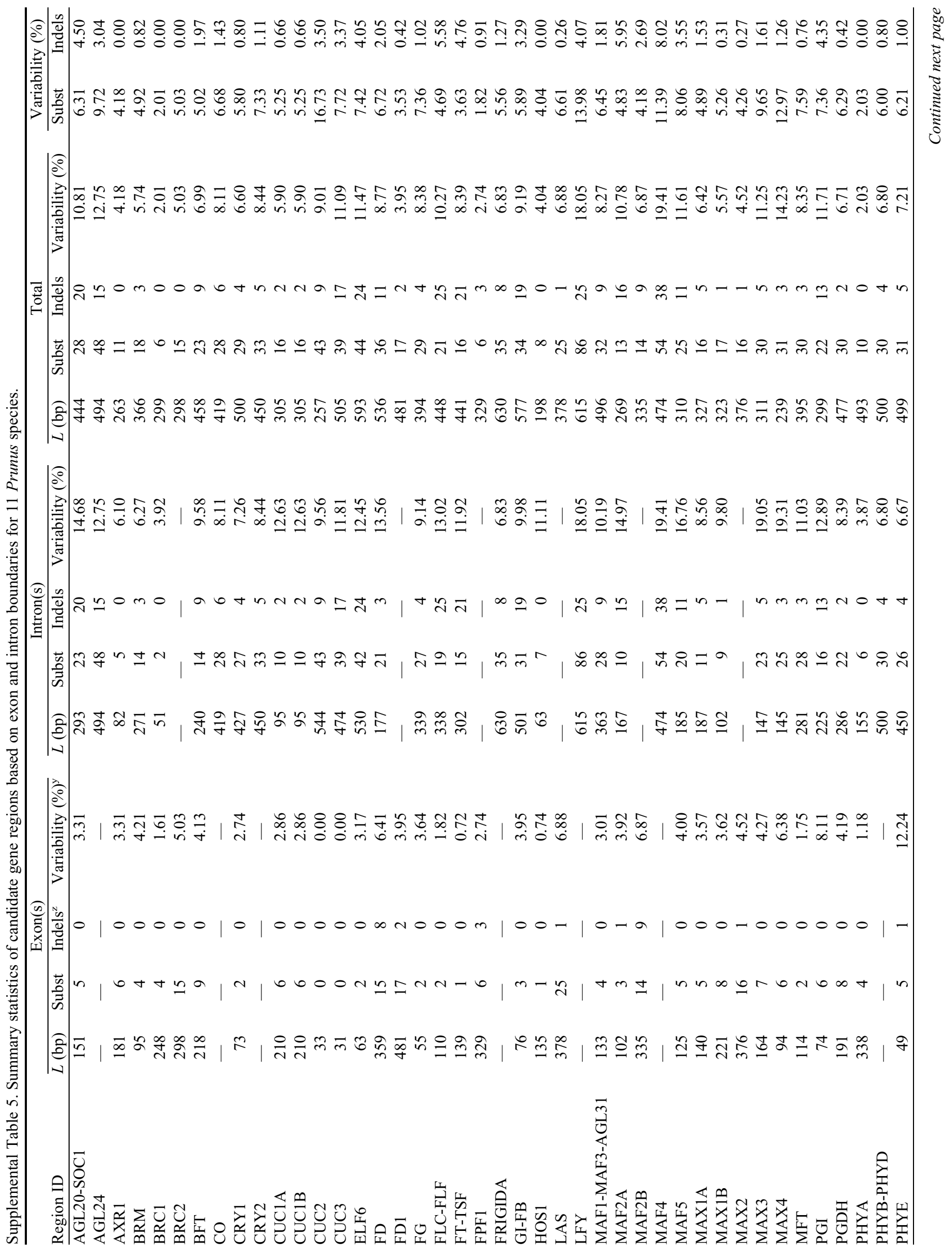




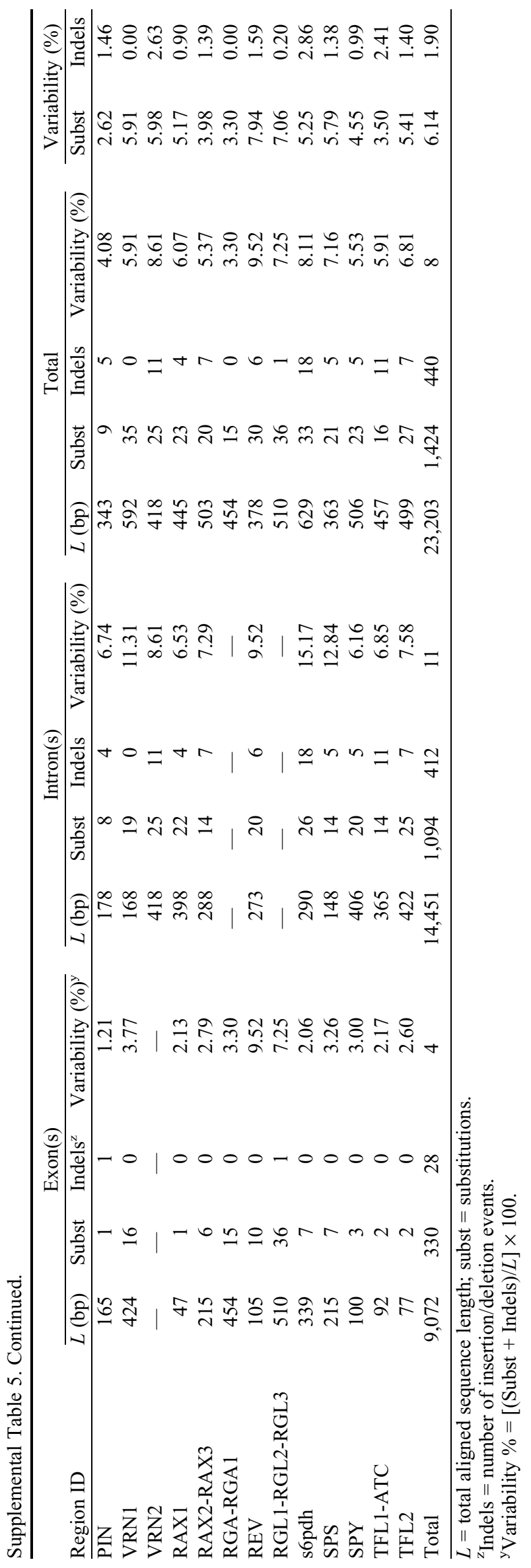




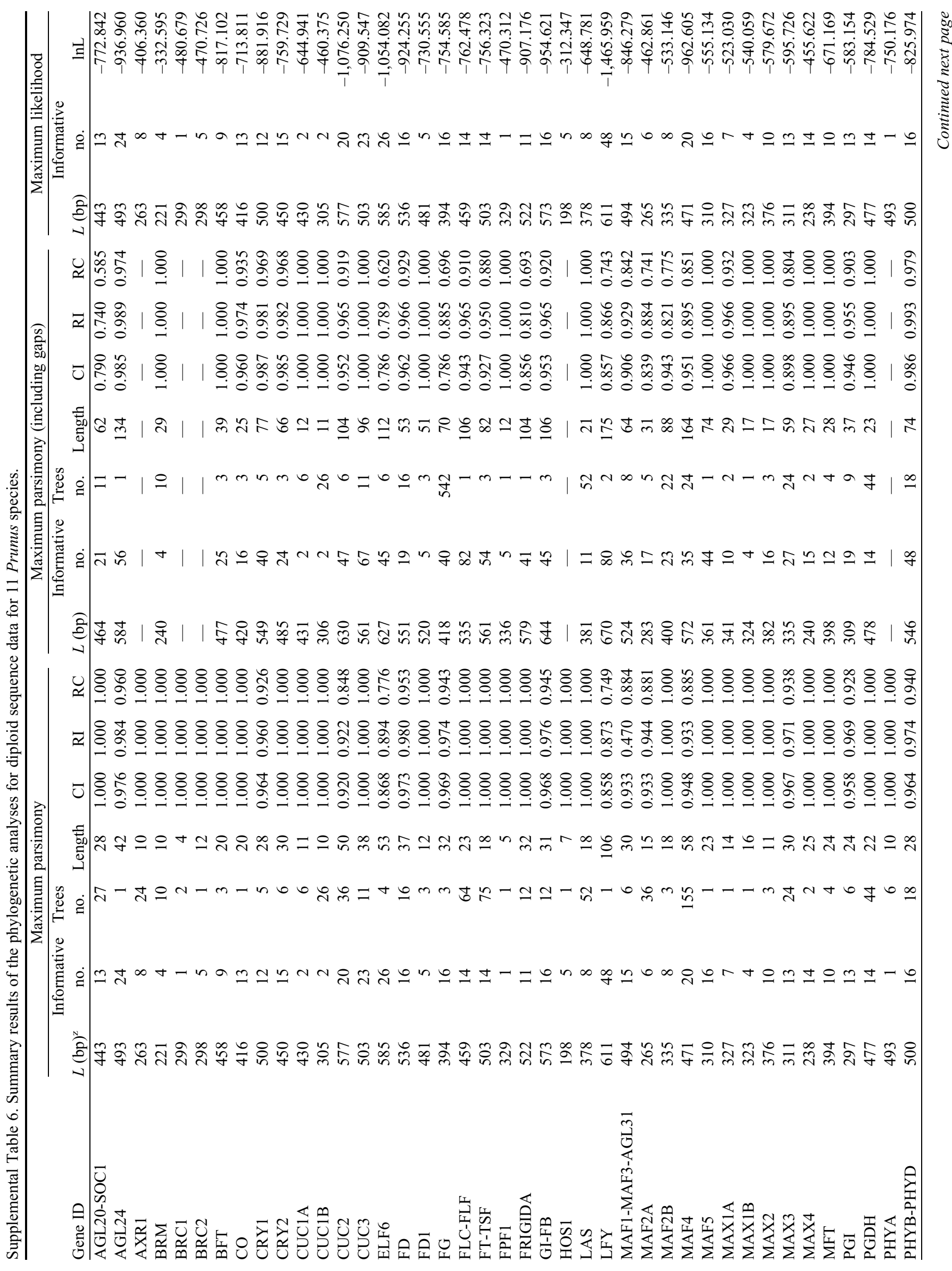




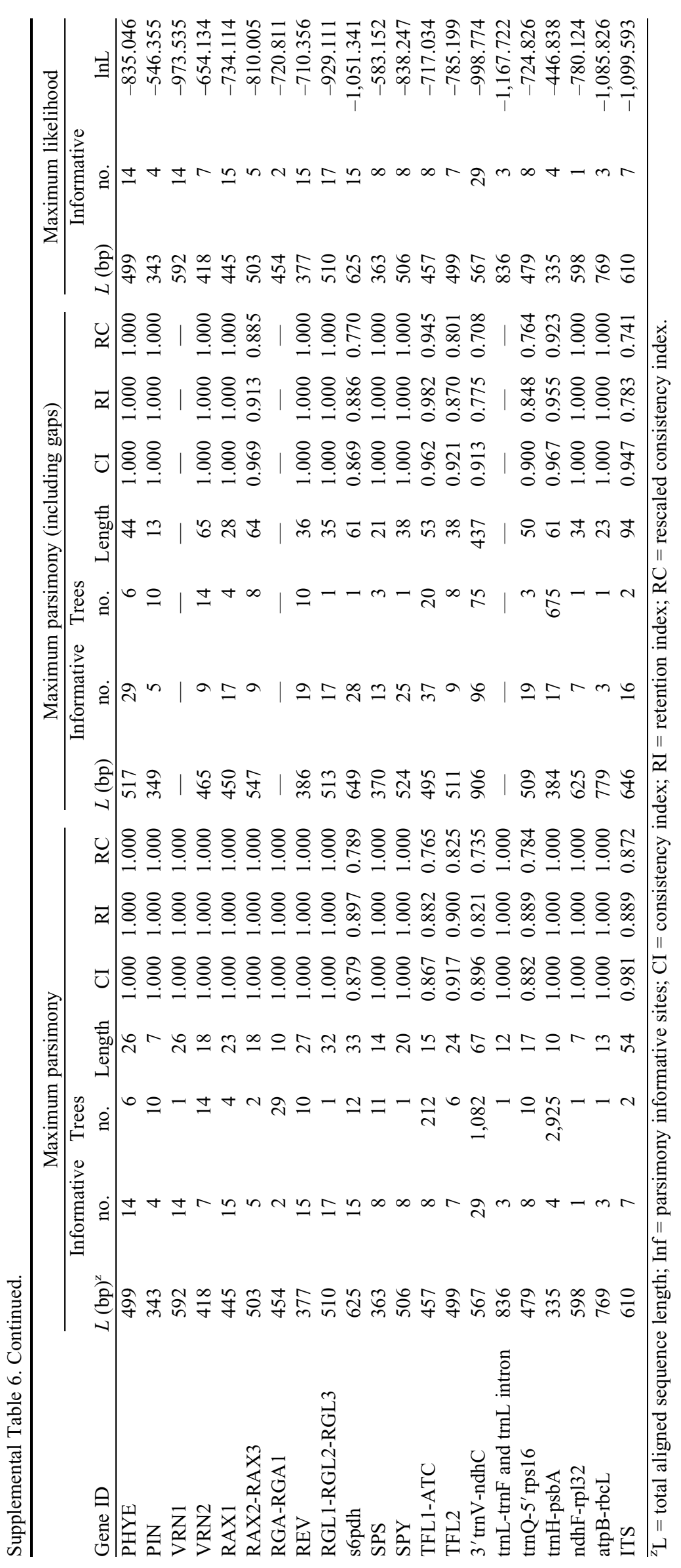




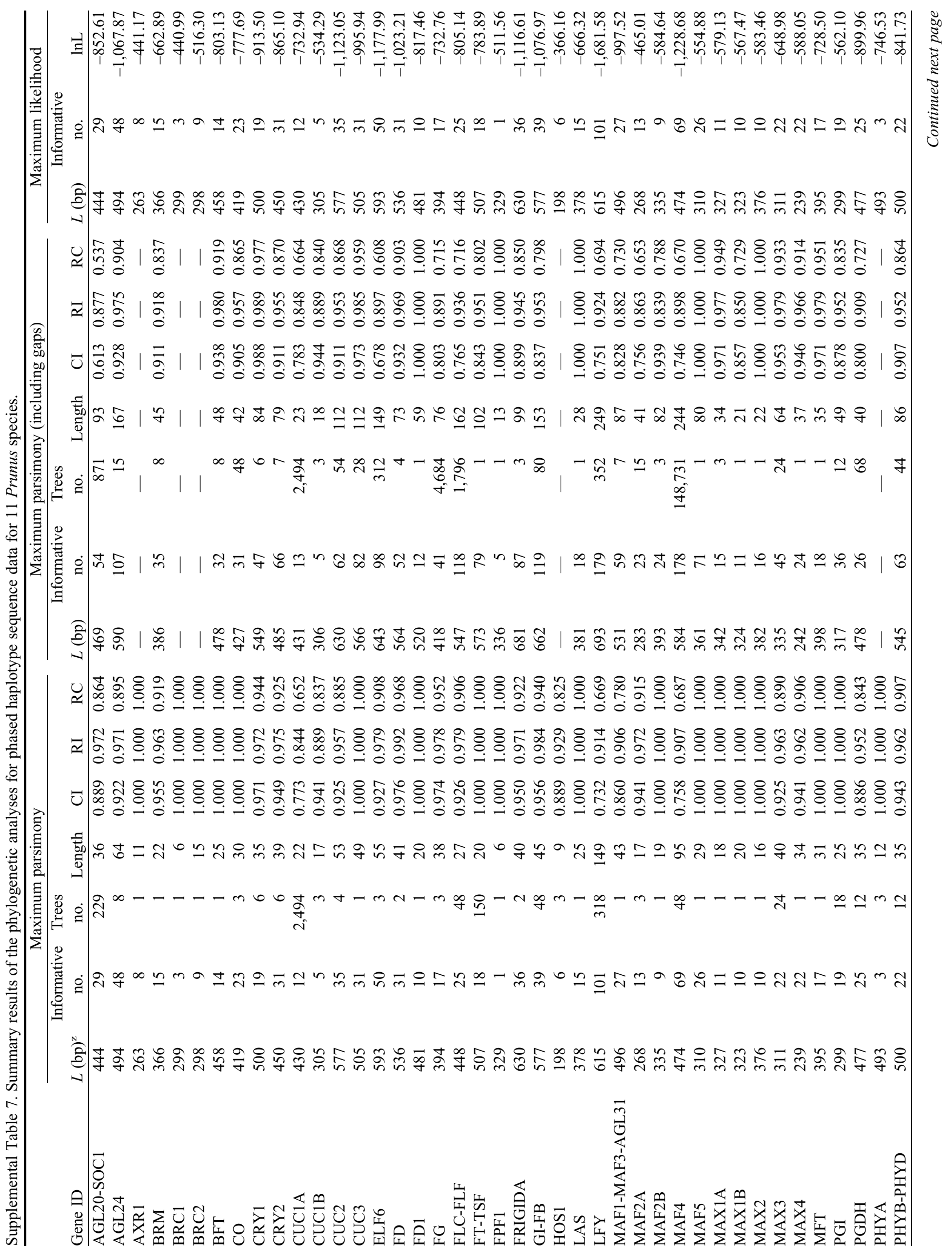




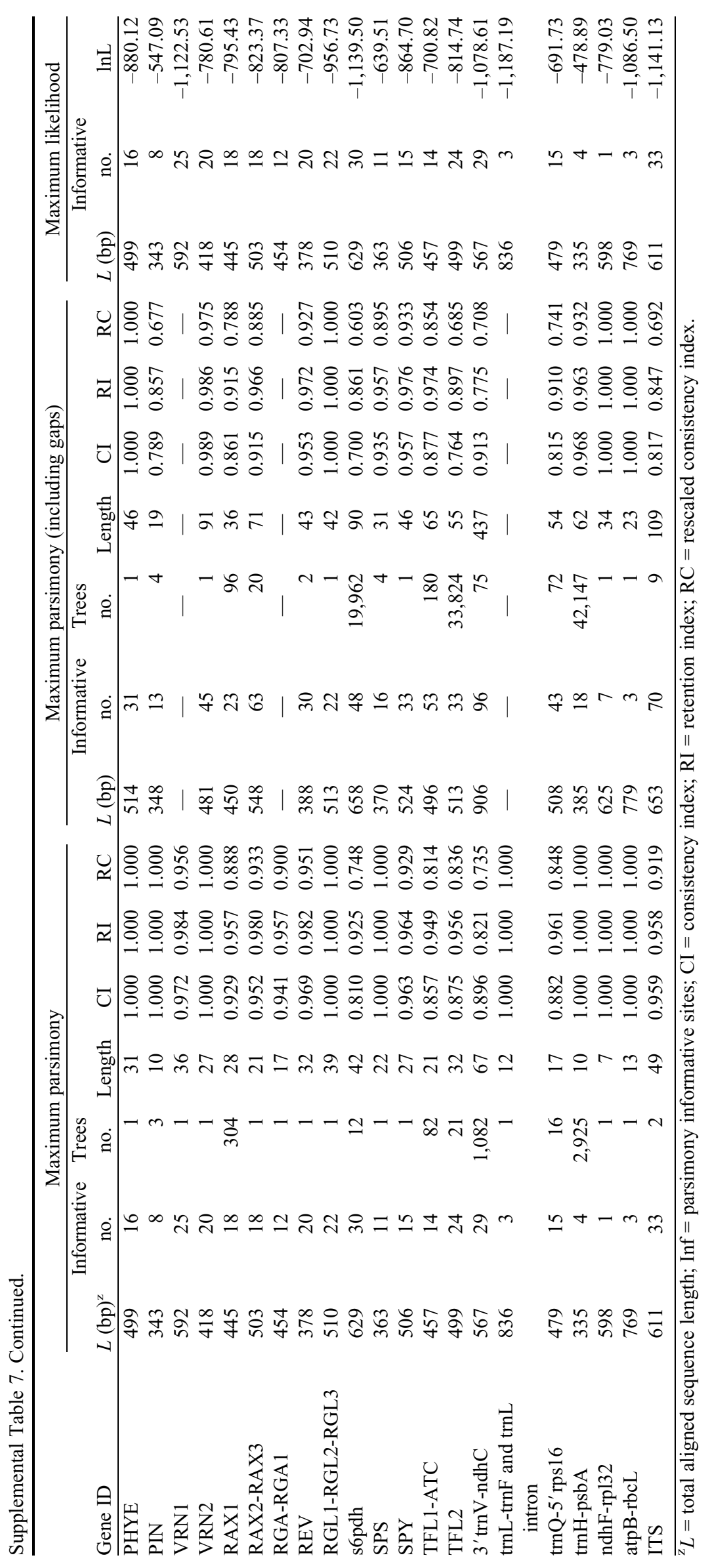




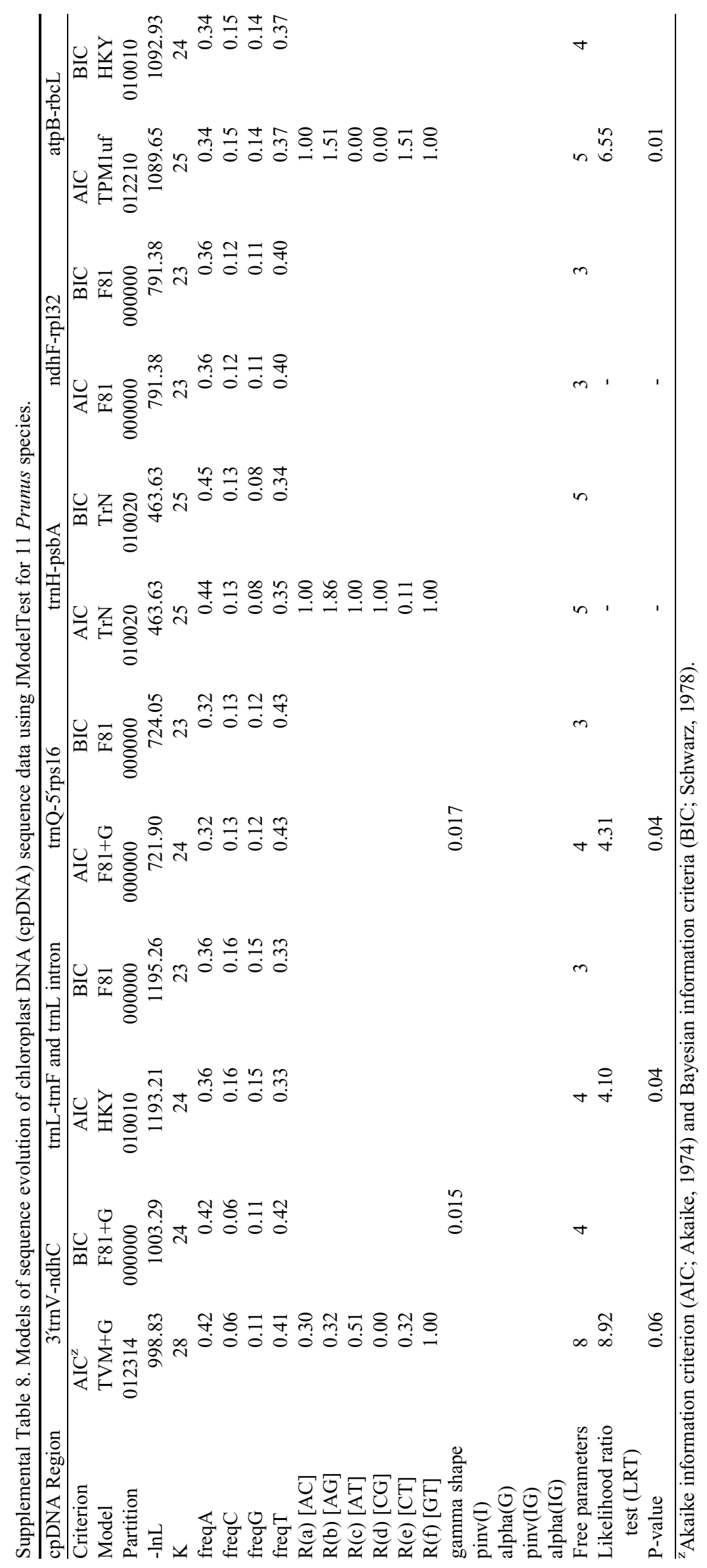




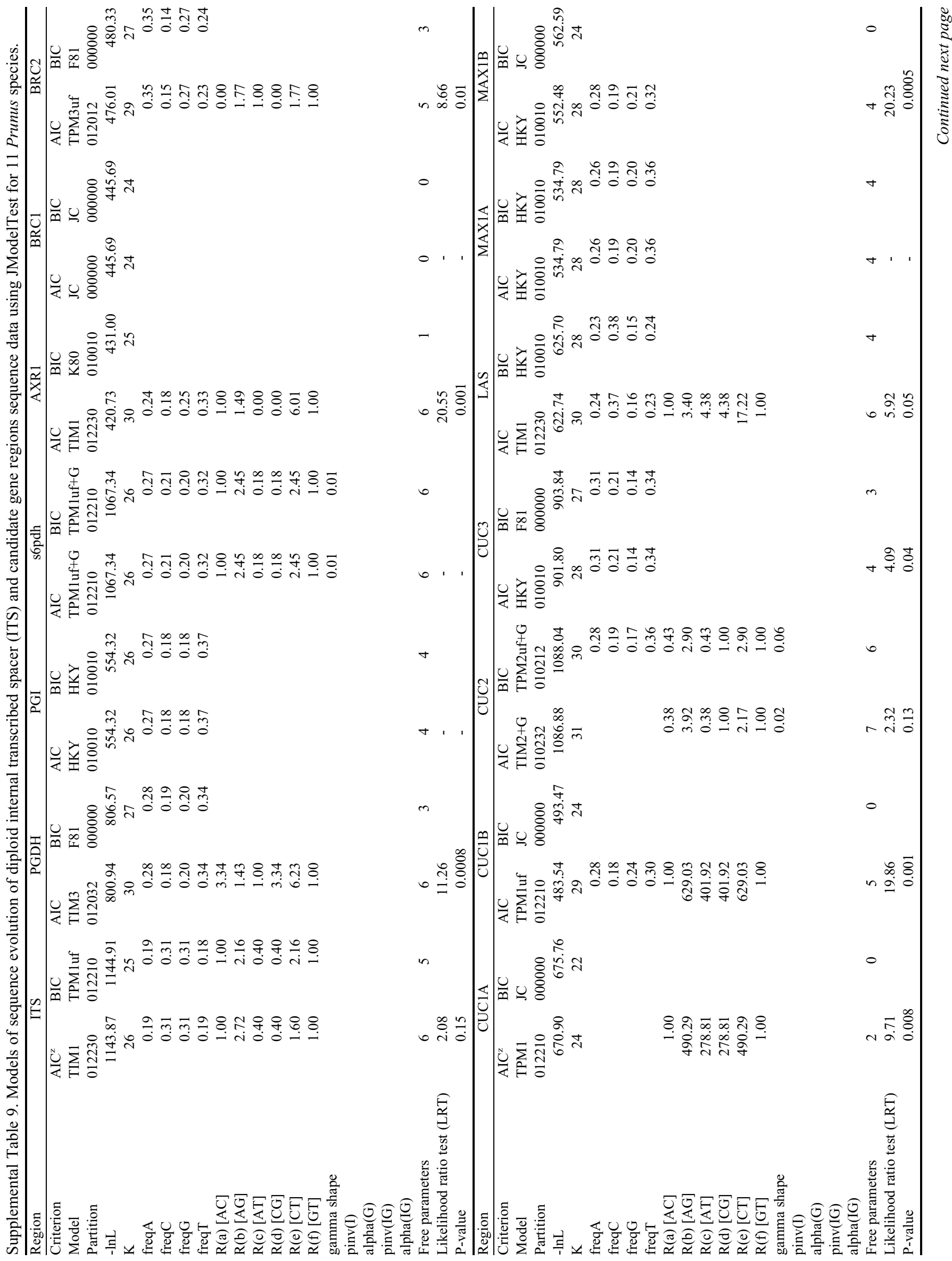




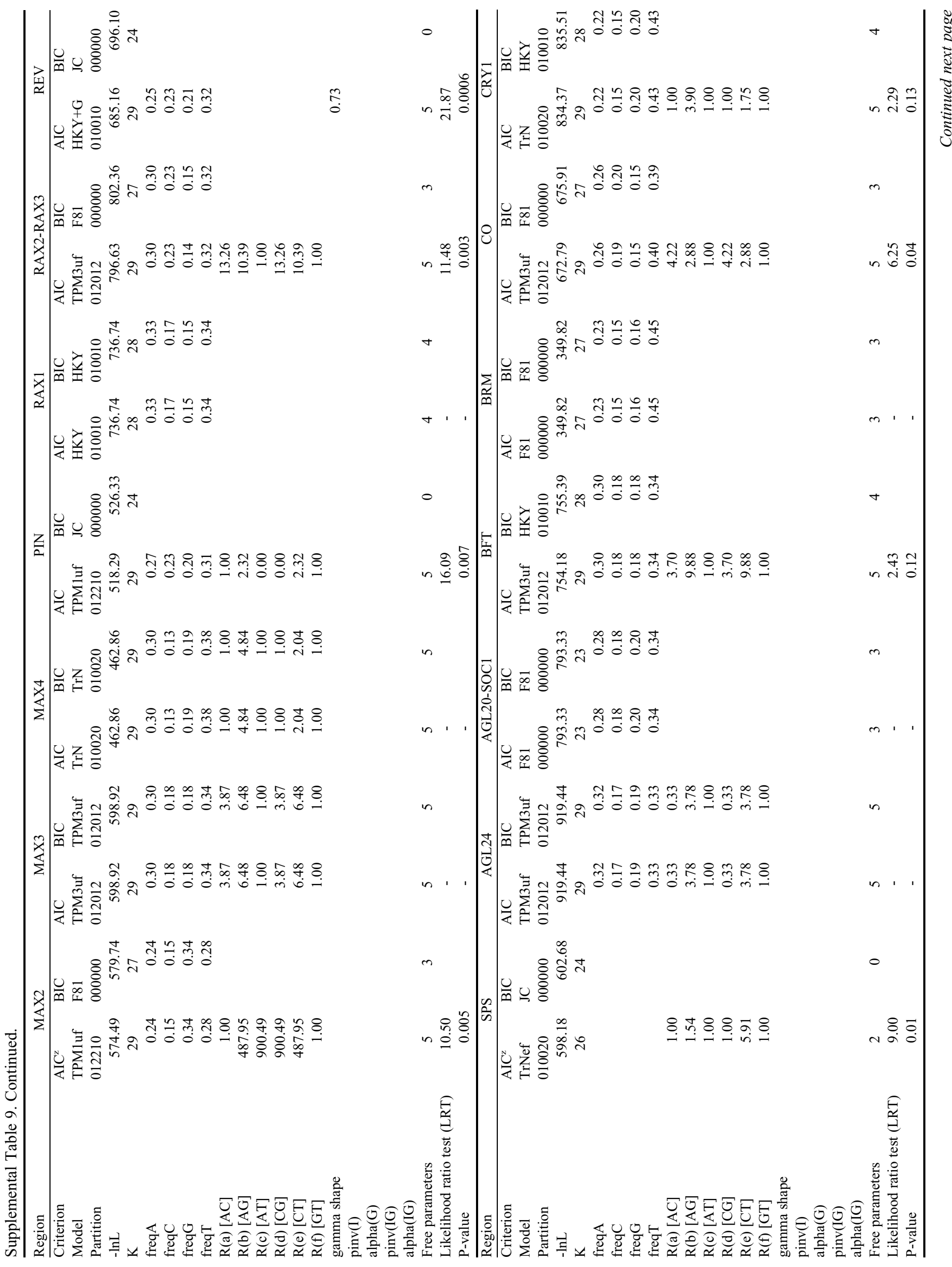




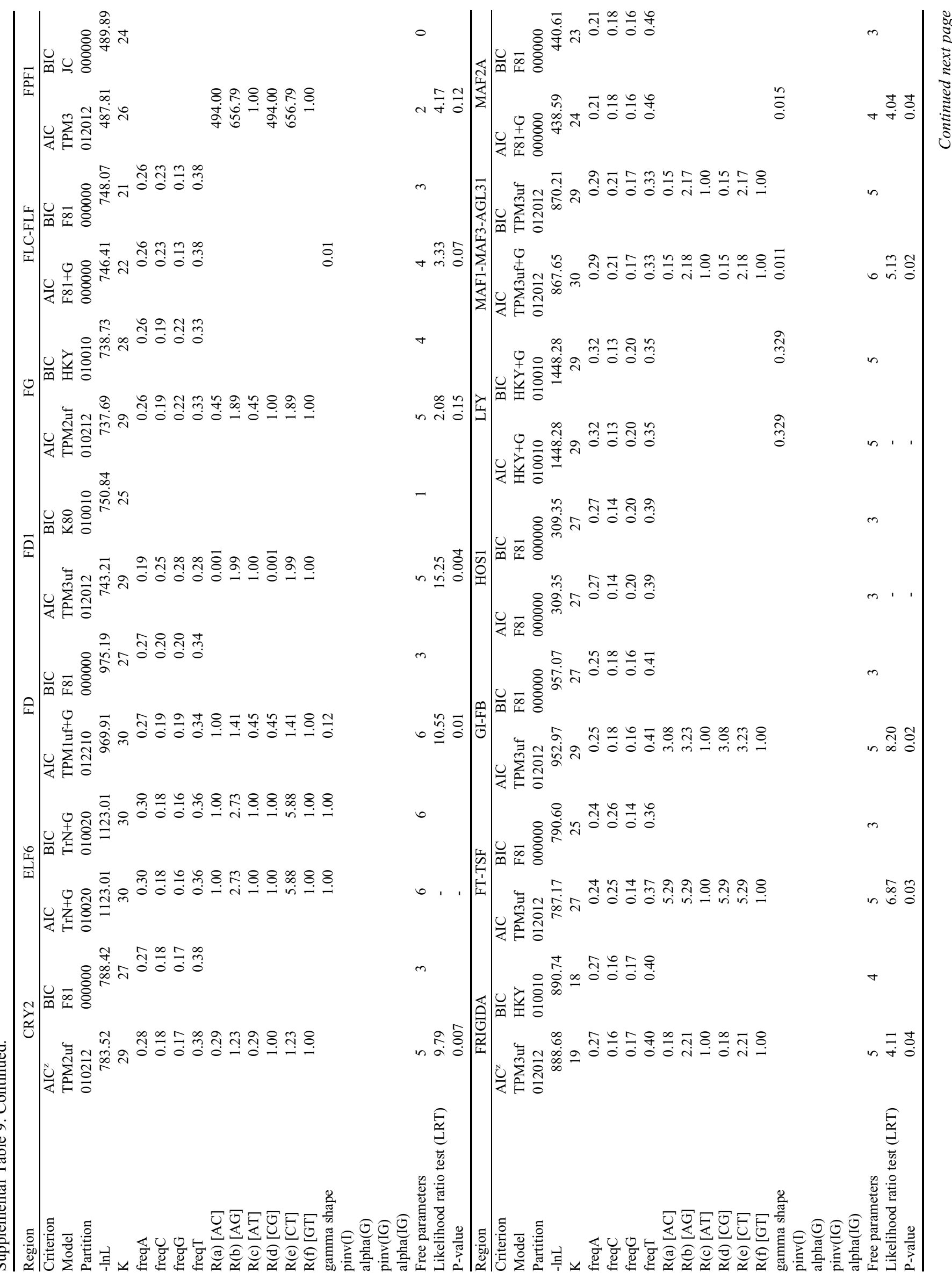




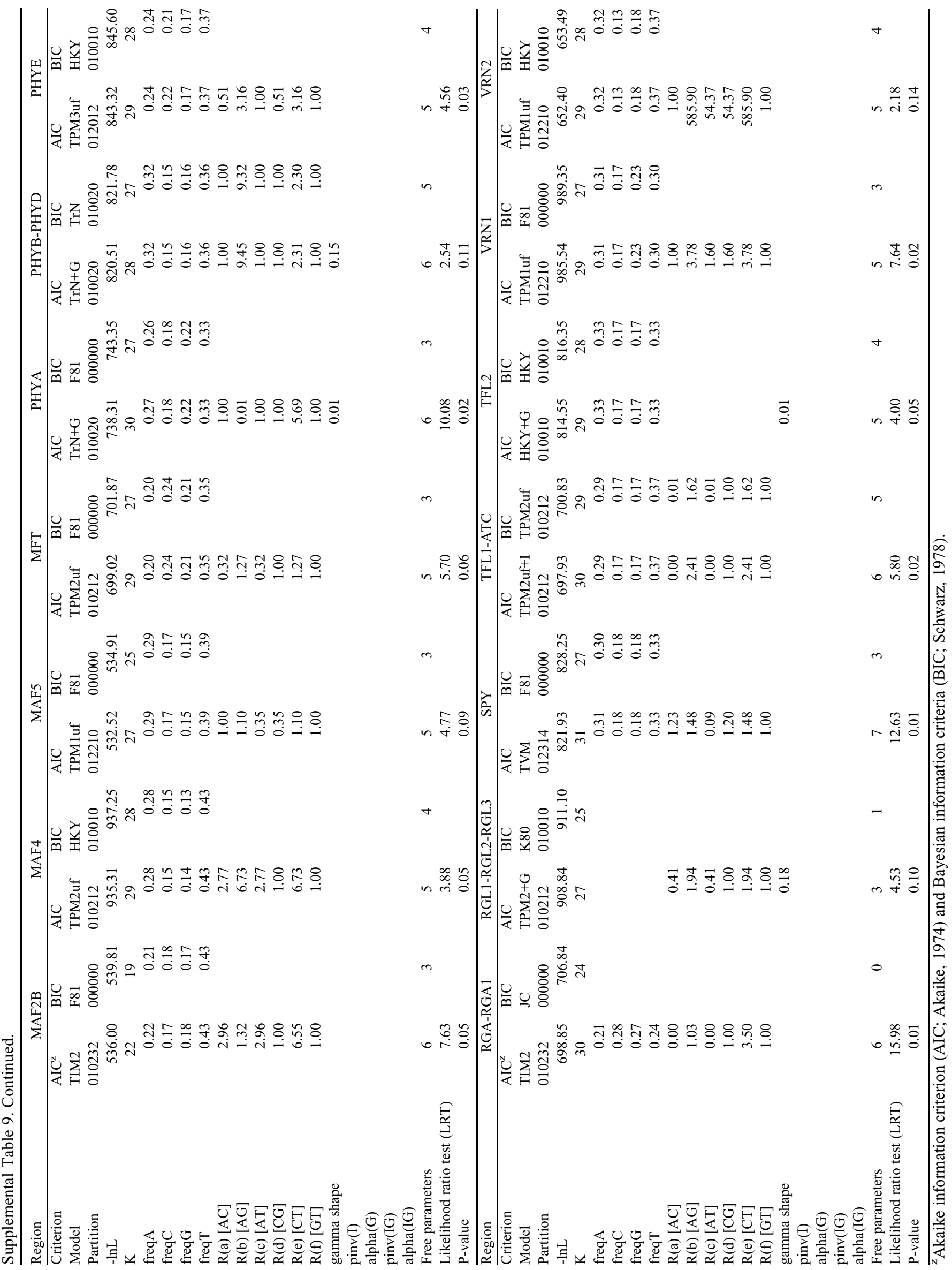




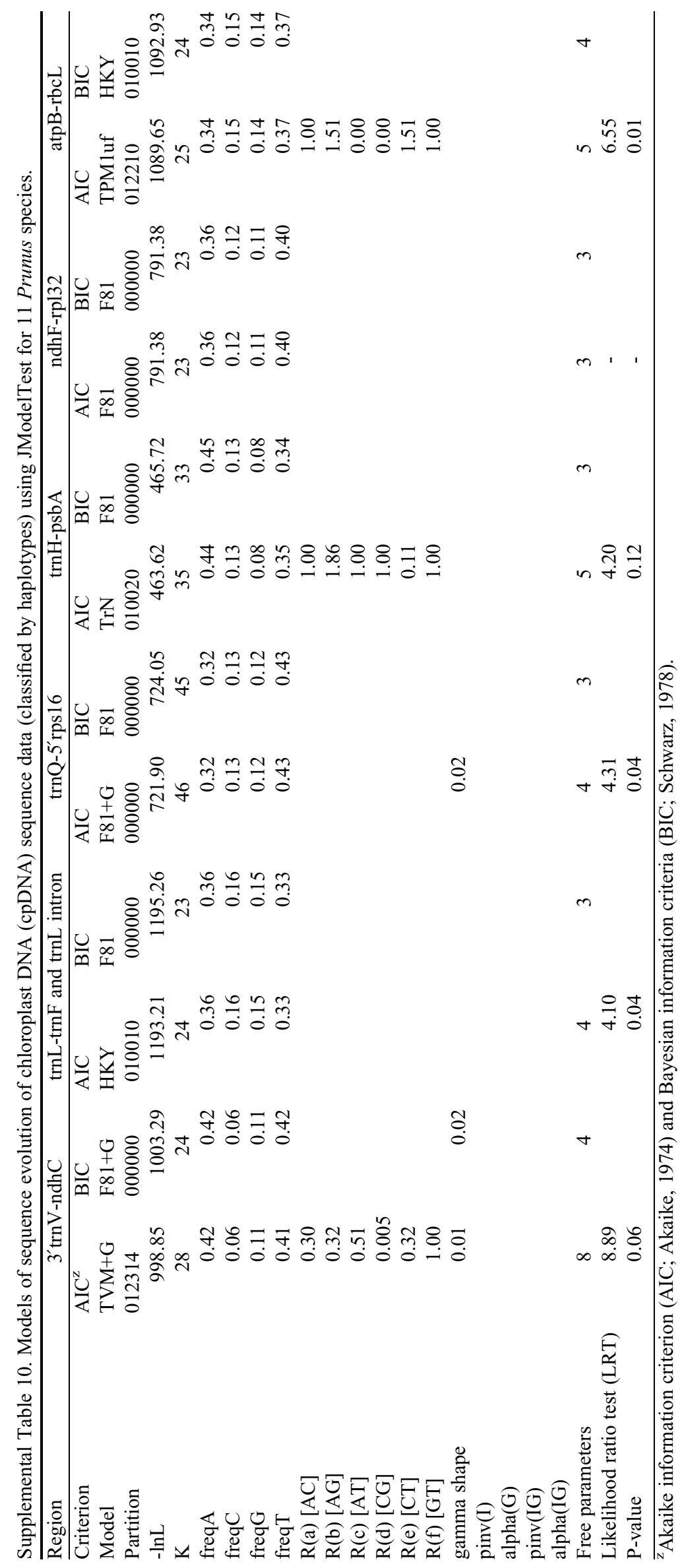




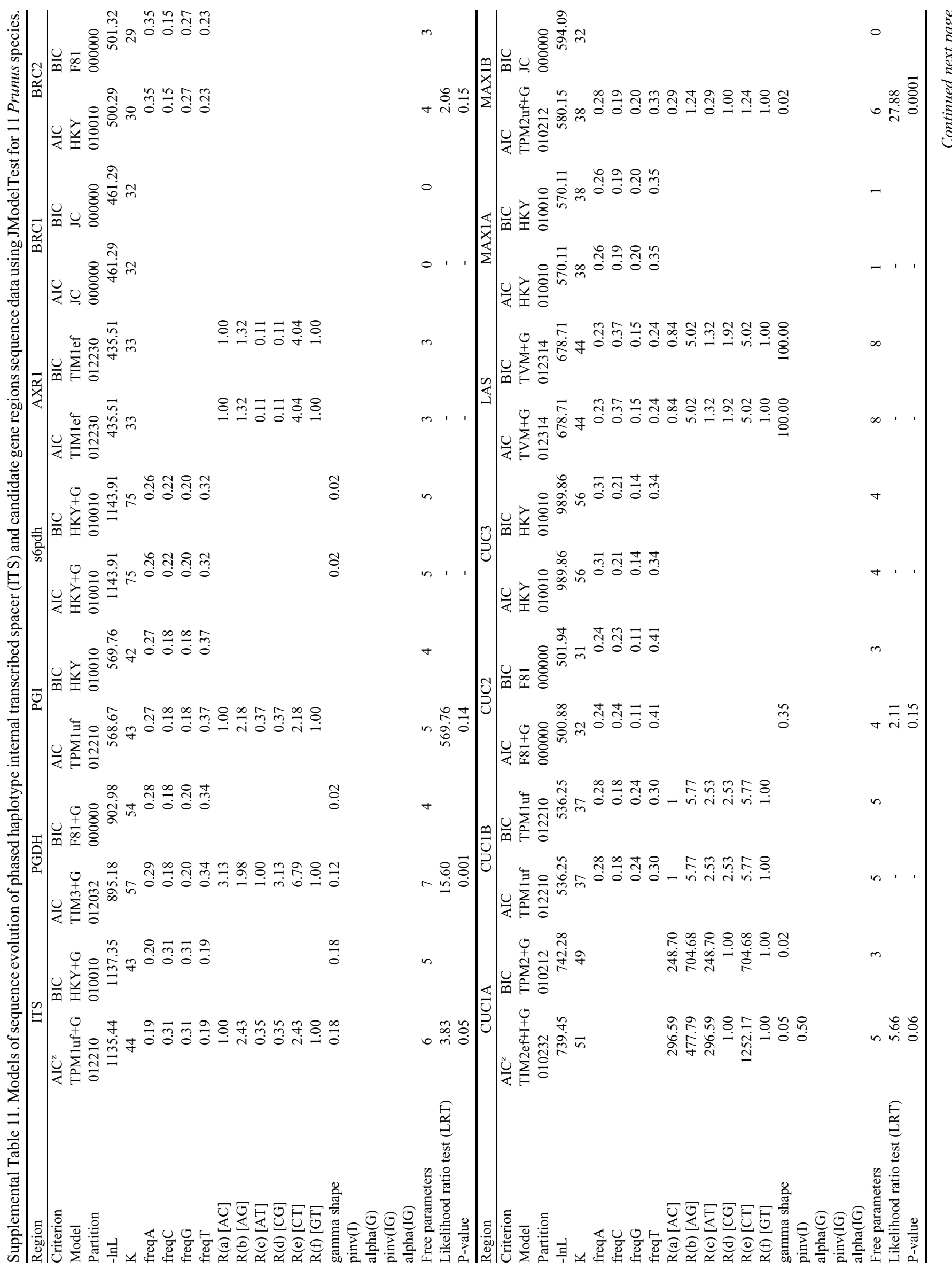




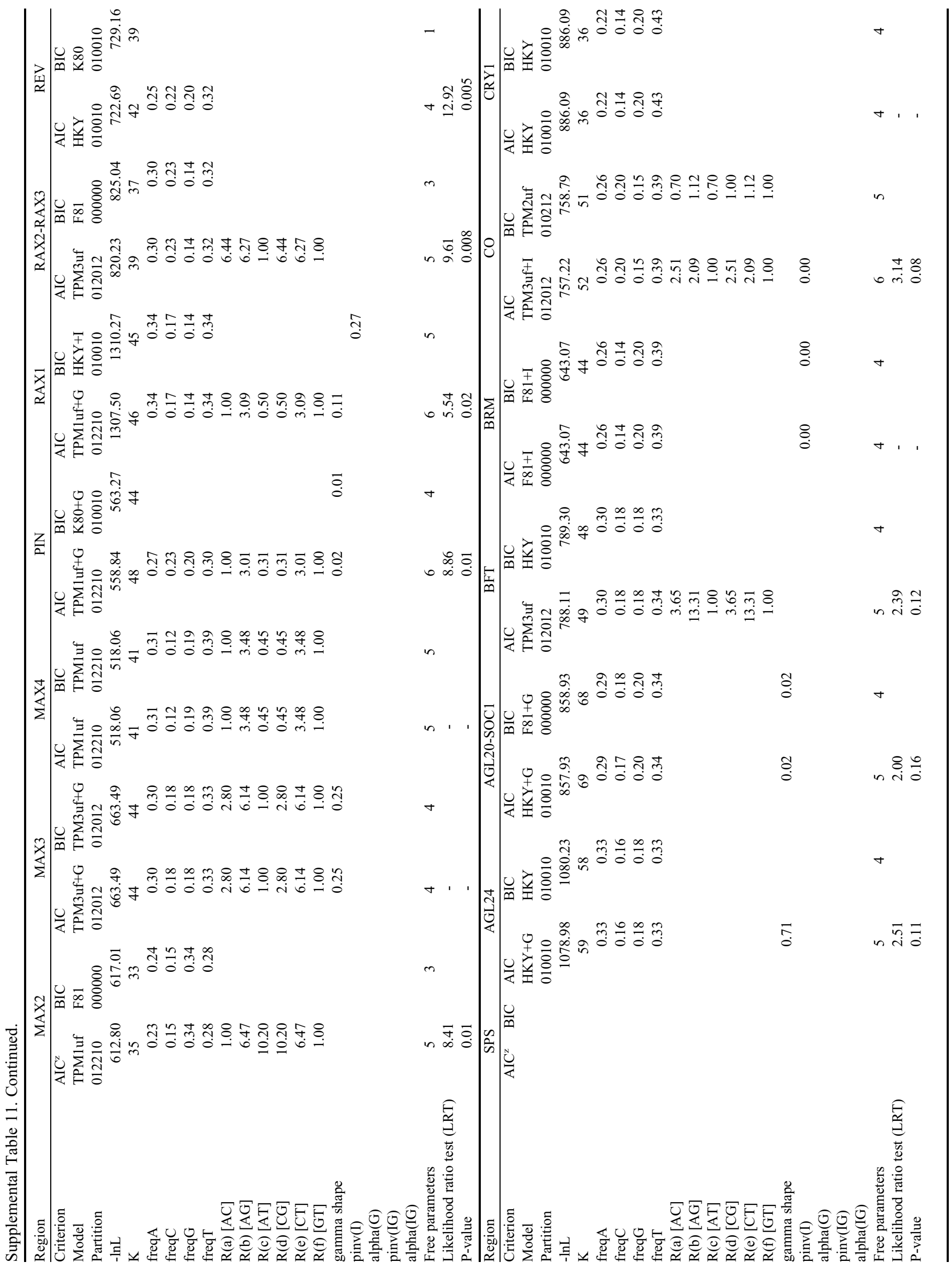




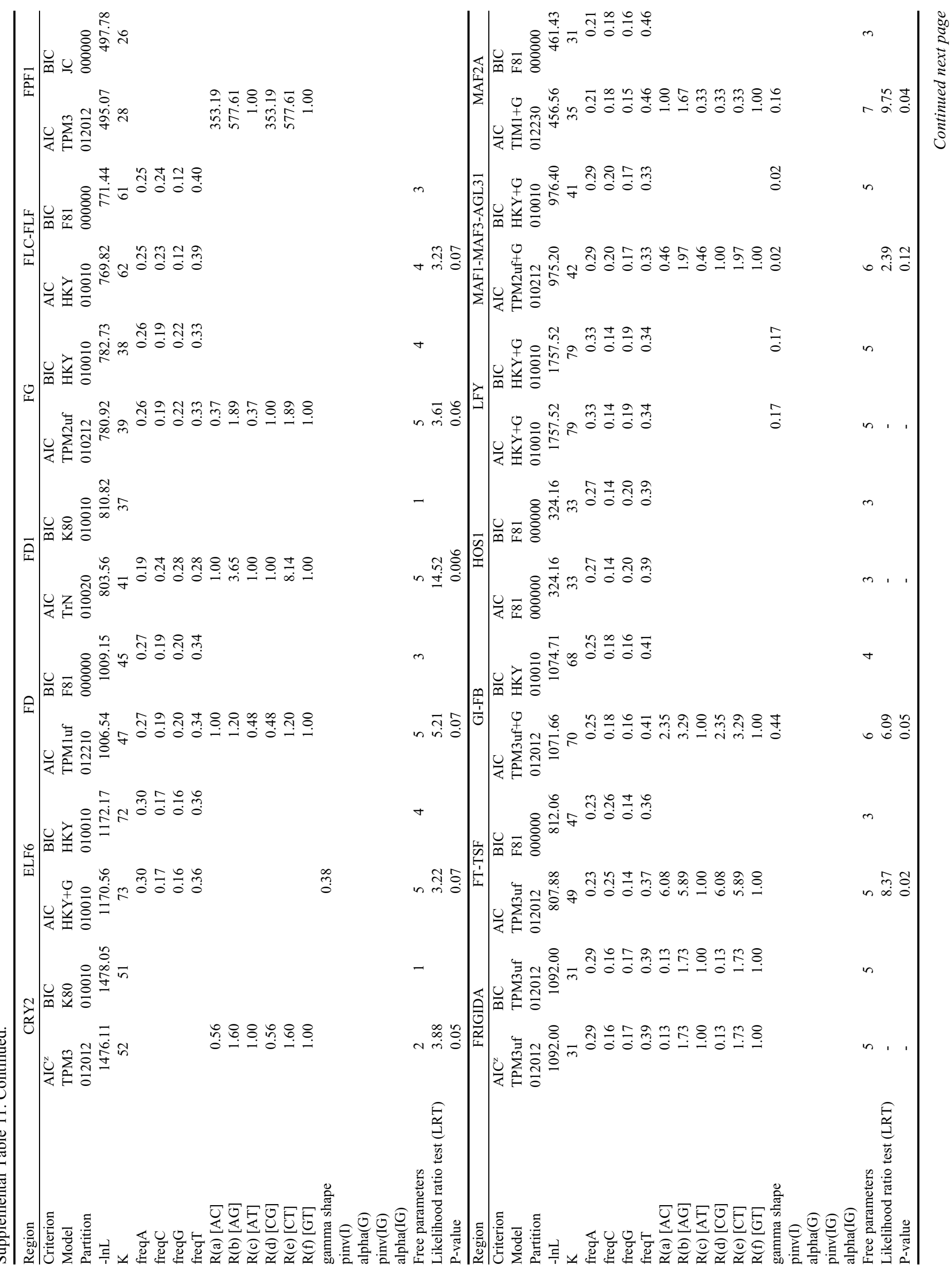




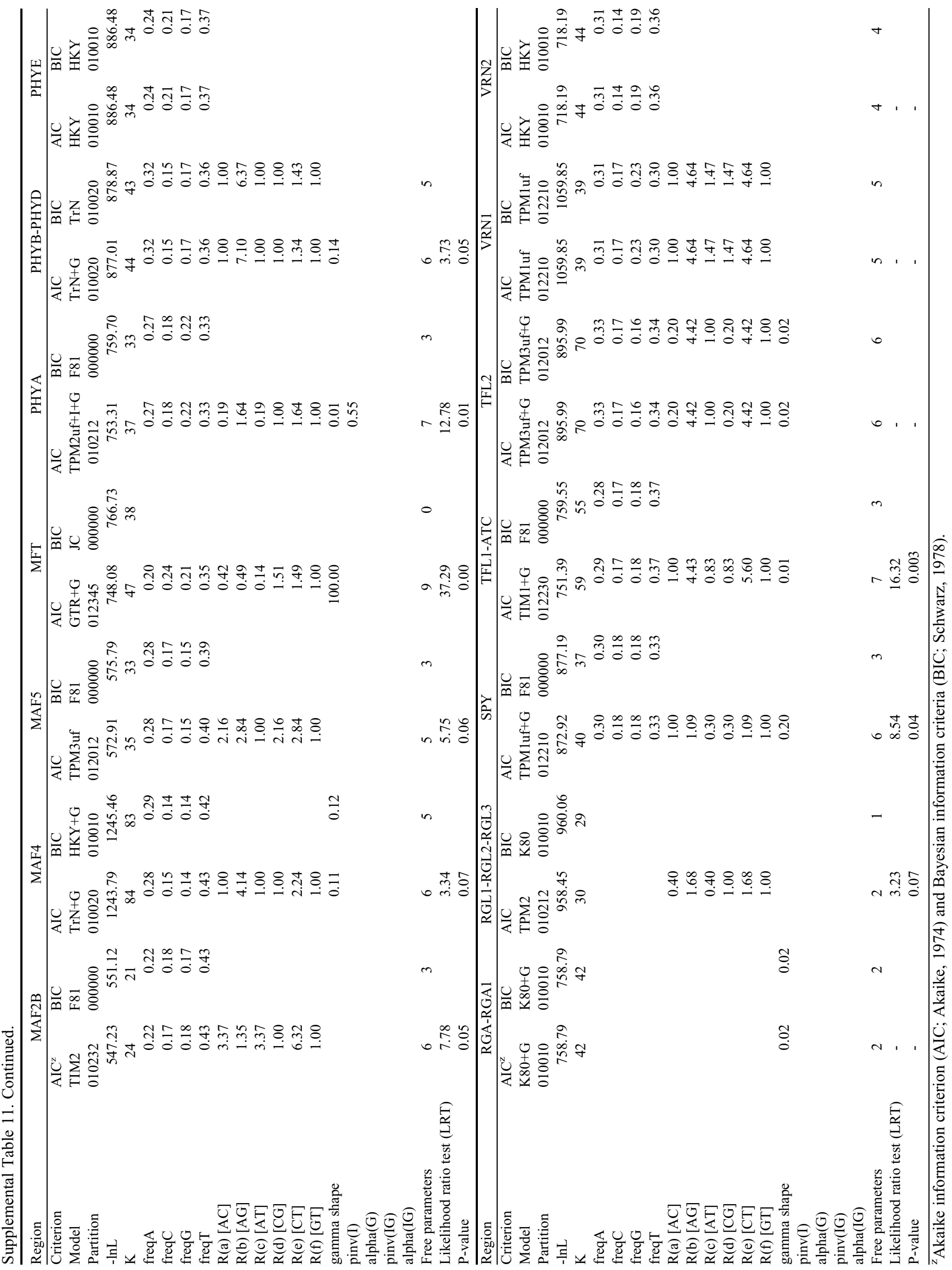

